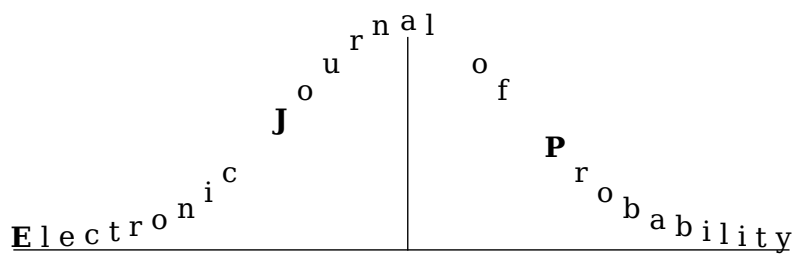

Electron. J. Probab. 26 (2021), article no. 68, 1-81.

ISSN: 1083-6489 https://doi.org/10.1214/21-EJP637

\title{
Zeros of smooth stationary Gaussian processes*
}

\author{
Michele Ancona $^{\dagger} \quad$ Thomas Letendre $^{\ddagger}$
}

\begin{abstract}
Let $f: \mathbb{R} \rightarrow \mathbb{R}$ be a stationary centered Gaussian process. For any $R>0$, let $\nu_{R}$ denote the counting measure of $\{x \in \mathbb{R} \mid f(R x)=0\}$. Under suitable assumptions on the regularity of $f$ and the decay of its correlation function at infinity, we derive the asymptotics as $R \rightarrow+\infty$ of the central moments of the linear statistics of $\nu_{R}$. In particular, we derive an asymptotics of order $R^{\frac{p}{2}}$ for the $p$-th central moment of the number of zeros of $f$ in $[0, R]$. As an application, we prove a functional Law of Large Numbers and a functional Central Limit Theorem for the random measures $\nu_{R}$. More precisely, after a proper rescaling, $\nu_{R}$ converges almost surely towards the Lebesgue measure in weak-* sense. Moreover, the fluctuation of $\nu_{R}$ around its mean converges in distribution towards the standard Gaussian White Noise. The proof of our moments estimates relies on a careful study of the $k$-point function of the zero point process of $f$, for any $k \geqslant 2$. Our analysis yields two results of independent interest. First, we derive an equivalent of this $k$-point function near any point of the large diagonal in $\mathbb{R}^{k}$, thus quantifying the short-range repulsion between zeros of $f$. Second, we prove a clustering property which quantifies the long-range decorrelation between zeros of $f$.
\end{abstract}

Keywords: central limit theorem; central moments; clustering; Gaussian process; Kac-Rice formula; law of large numbers; $k$-point function.

MSC2020 subject classifications: 60F05; 60F15; 60F17; 60F25; 60G15; 60G55; 60G57.

Submitted to EJP on September 1, 2020, final version accepted on April 27, 2021.

\section{Contents}

\section{Introduction}

1.1 Linear statistics associated with the zeros of a Gaussian process . . . . . . 3

1.2 Moments asymptotics . . . . . . . . . . . . . . 3

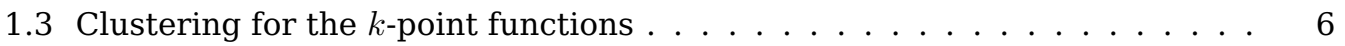

1.4 Law of large numbers and central limit theorem . . . . . . . . . . . 8

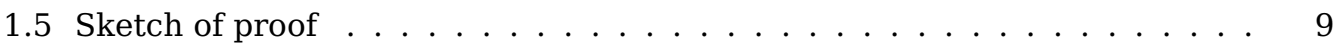

1.6 Related works . . . . . . . . . . . . . . . . . . . . . . 12

1.7 Organization of the paper . . . . . . . . . . . . . . . . . 14

*This work was supported by the French National Research Agency grants UniRaNDom (ANR-17-CE40-0008) and SpInQS (ANR-17-CE40-0011) and by the Israeli Science Foundation Grants 382/15 and 501/18.

${ }^{\dagger}$ Tel Aviv University, School of Mathematical Sciences. E-mail: michi .ancona@gmail .com.

${ }^{\ddagger}$ Université Paris-Saclay, CNRS, Laboratoire de Mathématiques d’Orsay. E-mail: letendre@math. cnrs.fr. 
2 Framework $\quad 15$

2.1 Partitions, products and diagonal inclusions . . . . . . . . . . . 15

2.2 Stationary Gaussian processes and correlation functions . . . . . . . . . 16

2.3 Zeros of stationary Gaussian processes . . . . . . . . . . . . . 18

3 Kac-Rice formulas and mean number of zeros 19

3.1 Kac-Rice formulas . . . . . . . . . . . . . . . . . . 19

3.2 Kac-Rice density and $k$-point functions . . . . . . . . . . . . . . 21

3.3 Proof of Proposition 1.8: expectation of the linear statistics . . . . . . . . 22

4 Proof of Proposition 1.11: asymptotics of the covariances 23

4.1 Asymptotics of the covariances . . . . . . . . . . . . . 23

4.2 Positivity of the leading constant . . . . . . . . . . . . . 25

5 Divided differences $\quad \mathbf{2 8}$

5.1 Hermite interpolation and divided differences . . . . . . . . . . . 28

5.2 Properties of the divided differences . . . . . . . . . . . . . 31

5.3 Double divided differences and correlation function $\ldots \ldots . \ldots 33$

6 Kac-Rice densities revisited and clustering 36

6.1 Graph partitions . . . . . . . . . . . . . . . . . . 37

6.2 Kac-Rice densities revisited . . . . . . . . . . . . . . . . . . . 39

6.3 Proof of Theorem 1.13: vanishing order of the $k$-point function . . . . . . 45

6.4 Variance and covariance matrices . . . . . . . . . . . . . 46

6.5 Denominator clustering . . . . . . . . . . . . . . . . . 49

6.6 Numerator clustering . . . . . . . . . . . . . . . . . . . . . . 51

6.7 Proof of Theorem 1.14: clustering for $k$-point functions . . . . . . . . . 55

7 Proof of Theorem 1.6: central moments asymptotics $\quad 57$

7.1 An integral expression of the central moments . . . . . . . . . . . . . 58

7.2 An upper bound on the contribution of each piece . . . . . . . . . . 60

7.3 Contribution of the partitions with an isolated point . . . . . . . 63

7.4 Contribution of the partitions into pairs . . . . . . . . . . 65

7.5 Conclusion of the proof . . . . . . . . . . . . 66 66

8 Limit theorems $\quad \mathbf{7 0}$

8.1 Proof of Theorem 1.16: law of large numbers . . . . . . . . . . . . 70

8.2 Proof of Theorem 1.21: central limit theorem . . . . . . . . . . . . . 71

A Examples of smooth non-degenerate processes

A.1 Spectral measure . . . . . . . . . . . . . . . . . . . 72

A.2 Non-degeneracy, spectral measure and ergodicity . . . . . . . . . . . 73

A.3 Smooth non-degenerate processes with fast-decreasing correlations . . . . 74

$\begin{array}{ll}\text { B Properties of the density function } F & \mathbf{7 6}\end{array}$

B.1 Proof of Lemma $4.2 \ldots \ldots \ldots \ldots \ldots$

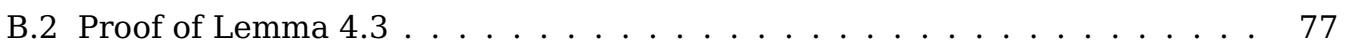

$\begin{array}{lr}\text { C A Gaussian lemma } & \mathbf{7 8}\end{array}$

$\begin{array}{lr}\text { References } & 79\end{array}$ 


\section{Introduction}

Let $Z$ denote the zero set of a smooth centered stationary Gaussian process $f$ on $\mathbb{R}$. A classical problem in probability is to understand the number of zeros of $f$ in a growing interval, that is the asymptotics of $\operatorname{Card}(Z \cap[0, R])$ as $R \rightarrow+\infty$. This problem has a long history, starting with the articles of Kac [23] and Rice [33] who computed the mean number of zeros of $f$ in an interval. We refer to Section 1.6 below for further discussion of related works.

In this paper, we compute the large $R$ asymptotics of the central moments of any order of $\operatorname{Card}(Z \cap[0, R])$, under suitable conditions on $f$. The starting point of our analysis is the Kac-Rice formula, which allows to write the $k$-th factorial moment of $\operatorname{Card}(Z \cap[0, R])$ as the integral over $[0, R]^{k}$ of the $k$-point function of the random point process $Z$. Most of the paper is devoted to the study of this $k$-point function $\rho_{k}$, that we believe to be of independent interest. A priori, $\rho_{k}$ is only well-defined on $\mathbb{R}^{k} \backslash \Delta_{k}$, where $\Delta_{k}$ denotes the large diagonal in $\mathbb{R}^{k}$. We prove that $\rho_{k}$ vanishes along $\Delta_{k}$, which is the sign of a repulsion between the zeros of $f$. In fact, we characterize this repulsion by deriving an equivalent of $\rho_{k}(x)$ as $x \rightarrow y$, for any $y \in \Delta_{k}$. We also prove that $\rho_{k}$ satisfies a clustering property if the correlation function of the process $f$ decays fast enough. This clustering property can be interpreted as a clue that zeros of $f$ in two disjoint intervals that are far from one another are quasi-independent. Our main tool in the study of $\rho_{k}$ and its singularities are the divided differences associated with $f$. We believe that the methods we develop below regarding these divided differences can have applications beyond the scope of this paper.

\subsection{Linear statistics associated with the zeros of a Gaussian process}

Let us introduce quickly the object of our study. More details are given in Section 2 below. Let $f: \mathbb{R} \rightarrow \mathbb{R}$ be a stationary centered Gaussian process of class $\mathcal{C}^{1}$. Let $\kappa: x \mapsto \mathbb{E}[f(0) f(x)]$ denote the correlation function of $f$. We assume that $f$ is normalized so that $\kappa(0)=1=-\kappa^{\prime \prime}(0)$ (see Section 2.2). The zero set $Z=f^{-1}(0)$ is then almost surely a closed discrete subset of $\mathbb{R}$ (see Lemma 2.12).

We denote by $\nu=\sum_{x \in Z} \delta_{x}$ the counting measure of $Z$, where $\delta_{x}$ is the unit Dirac mass at $x$. Let $\phi: \mathbb{R} \rightarrow \mathbb{R}$, we denote by $\langle\nu, \phi\rangle=\sum_{x \in Z} \phi(x)$ whenever this makes sense. Besides, for any $R>0$, we denote by $\phi_{R}: x \mapsto \phi\left(\frac{x}{R}\right)$. Finally, for any $A \subset \mathbb{R}$, we denote by $\mathbb{1}_{A}$ the indicator function of $A$. Then, for any $R>0$, we have:

$$
\operatorname{Card}(Z \cap[0, R])=\left\langle\nu, \mathbb{1}_{[0, R]}\right\rangle=\left\langle\nu,\left(\mathbb{1}_{[0,1]}\right)_{R}\right\rangle
$$

More generally, we can consider the asymptotics of $\left\langle\nu, \phi_{R}\right\rangle$ as $R \rightarrow+\infty$, where $\phi: \mathbb{R} \rightarrow \mathbb{R}$ is a nice enough test-function. It turns out that the dual point of view is more relevant, and this is the one we adopt in this paper: instead of integrating $\phi_{R}$ over $Z$, we consider the integral of a fixed test-function $\phi$ over homothetical copies of $Z$. Let $R>0$, we denote by $Z_{R}=\{x \in \mathbb{R} \mid f(R x)=0\}$ and by $\nu_{R}=\sum_{x \in Z_{R}} \delta_{x}$ its counting measure. Then, for all $\phi: \mathbb{R} \rightarrow \mathbb{R}$, we have $\left\langle\nu, \phi_{R}\right\rangle=\left\langle\nu_{R}, \phi\right\rangle$. In particular, $\operatorname{Card}(Z \cap[0, R])=\left\langle\nu_{R}, \mathbb{1}_{[0,1]}\right\rangle$. Quantities of the form $\left\langle\nu_{R}, \phi\right\rangle$ are called the linear statistics of $\nu_{R}$. In the following, we study the large $R$ asymptotic distribution of the random measure $\nu_{R}$, mostly through the central moments of its linear statistics.

\subsection{Moments asymptotics}

Our first theorem describes the large $R$ asymptotics of the central moments of the linear statistics $\left\langle\nu_{R}, \phi\right\rangle$ of the random measure $\nu_{R}$. To the best of our knowledge, this is the first result of this kind for Gaussian processes on $\mathbb{R}$, even in the simplest case of 
$\left\langle\nu_{R}, \mathbb{1}_{[0,1]}\right\rangle=\operatorname{Card}(Z \cap[0, R])$. We will consider the following quantities, that are slightly more general.

Definition 1.1 (Central moments). Let $p \geqslant 2$ be an integer and let $R>0$. For any test-functions $\phi_{1}, \ldots, \phi_{p}$, we denote by

$$
m_{p}\left(\nu_{R}\right)\left(\phi_{1}, \ldots, \phi_{p}\right)=\mathbb{E}\left[\prod_{i=1}^{p}\left(\left\langle\nu_{R}, \phi_{i}\right\rangle-\mathbb{E}\left[\left\langle\nu_{R}, \phi_{i}\right\rangle\right]\right)\right]
$$

whenever the right-hand side makes sense. In particular, $m_{p}\left(\nu_{R}\right)(\phi, \ldots, \phi)$ equals $m_{p}\left(\left\langle\nu_{R}, \phi\right\rangle\right)$, the $p$-th central moment of $\left\langle\nu_{R}, \phi\right\rangle$, when this quantity is well-defined. When $p=2$, we use the standard notation $\operatorname{Var}\left(\left\langle\nu_{R}, \phi\right\rangle\right)$ instead of $m_{2}\left(\left\langle\nu_{R}, \phi\right\rangle\right)$.

In this paper, we use the following class of test-functions. Note that this class contains, among others, the indicator functions of bounded intervals and the continuous functions decreasing fast enough at infinity.

Definition 1.2 (Test-function). We say that a measurable function $\phi: \mathbb{R} \rightarrow \mathbb{R}$ is a testfunction if $\phi$ is integrable, essentially bounded and continuous almost everywhere with respect to the Lebesgue measure.

In order to say something about central moments, we need to make some assumptions on the random process $f$. These assumptions are further discussed in Section 2.2, and in Appendix A where we build examples of processes satisfying these conditions. For now, let us just give one concrete example. The hypotheses of all the theorems in the present paper are satisfied by the so-called Bargmann-Fock process. This process is the centered stationary Gaussian process on $\mathbb{R}$ whose correlation function is $x \mapsto e^{-\frac{1}{2} x^{2}}$. See Appendix A for more details, especially Example A.4.

Notation 1.3 ( $\mathcal{C}^{k}$-norms). Let $k \in \mathbb{N}$ and let $g: \mathbb{R} \rightarrow \mathbb{R}$ be a $\mathcal{C}^{k}$-function such that $g$ and all its derivatives of order at most $k$ are bounded on $\mathbb{R}$. For any $\eta \geqslant 0$, we denote by:

$$
\|g\|_{k, \eta}=\sup \left\{\left|g^{(l)}(x)\right||0 \leqslant l \leqslant k,| x \mid \geqslant \eta\right\}
$$

where $g^{(l)}$ stands for the $l$-th derivative of $g$. If $\eta=0$, we simply denote $\|g\|_{k, 0}$ by $\|g\|_{k}$.

If $f$ is a $\mathcal{C}^{p}$-process, then its correlation function satisfies $\kappa \in \mathcal{C}^{2 p}(\mathbb{R})$. Moreover, by Cauchy-Schwarz's Inequality, $\kappa^{(k)}$ is bounded for all $k \in\{0, \ldots, 2 p\}$. Hence $\|\kappa\|_{k, \eta}$ is well-defined for any $k \in\{0, \ldots, 2 p\}$ and $\eta \geqslant 0$. We refer to Section 2.2 for more details.

Definition 1.4 (Partitions). Let $A$ be a non-empty finite set, a partition of the set $A$ is a family $\mathcal{I}=\left\{I_{1}, \ldots, I_{m}\right\}$ of non-empty disjoint subsets of $A$ such that $\bigsqcup_{i=1}^{m} I_{i}=A$. We denote by $\mathcal{P}_{A}$ (resp. $\mathcal{P}_{k}$ ) the set of partitions of $A$ (resp. $\{1, \ldots, k\}$ ). A partition into pairs of $A$ is a partition $\mathcal{I} \in \mathcal{P}_{A}$ such that $\operatorname{Card}(I)=2$ for all $I \in \mathcal{I}$. We denote by $\mathcal{P P}_{A},\left(\right.$ resp. $\mathcal{P P}_{k}$ ) the set of partitions into pairs of $A$ (resp. $\{1, \ldots, k\}$ ). We also use the convention that $\mathcal{P}_{\emptyset}=\{\emptyset\}=\mathcal{P} \mathcal{P}_{\emptyset}$.

Notation 1.5 (Gaussian moments). For all $p \in \mathbb{N}$, we denote by $\mu_{p}$ the $p$-th moment of a centered Gaussian variable of variance 1 . Recall that we have $\mu_{p}=\operatorname{Card}(\mathcal{P P})$, that is $\mu_{p}=2^{-\frac{p}{2}} p !\left(\frac{p}{2} !\right)^{-1}$ if $p$ is even, and $\mu_{p}=0$ if $p$ is odd.

Theorem 1.6 (Central moments asymptotics). Let $p \geqslant 2$ be an integer. Let $f$ be a normalized stationary centered Gaussian $\mathcal{C}^{p}$-process and let $\kappa$ denote its correlation function. We assume that, $\|\kappa\|_{p, \eta}=o\left(\eta^{-4 p}\right)$ as $\eta \rightarrow+\infty$. For all $R>0$, we denote by $\nu_{R}$ the counting measure of $\{x \in \mathbb{R} \mid f(R x)=0\}$.

Let $\phi_{1}, \ldots, \phi_{p}$ be test-functions in the sense of Definition 1.2. Then, as $R \rightarrow+\infty$, we 
have:

$$
m_{p}\left(\nu_{R}\right)\left(\phi_{1}, \ldots, \phi_{p}\right)=\sum_{\left\{\left\{a_{i}, b_{i}\right\} \mid 1 \leqslant i \leqslant \frac{p}{2}\right\} \in \mathcal{P} \mathcal{P}_{p}} \prod_{i=1}^{\frac{p}{2}} m_{2}\left(\nu_{R}\right)\left(\phi_{a_{i}}, \phi_{b_{i}}\right)+o\left(R^{\frac{p}{2}}\right) .
$$

In particular, if $\phi$ is a test-function then, as $R \rightarrow+\infty$, we have:

$$
m_{p}\left(\left\langle\nu_{R}, \phi\right\rangle\right)=\mu_{p} \operatorname{Var}\left(\left\langle\nu_{R}, \phi\right\rangle\right)^{\frac{p}{2}}+o\left(R^{\frac{p}{2}}\right) .
$$

Remark 1.7. If $p$ is odd, then $\mathcal{P P}_{p}=\emptyset$ and $\mu_{p}=0$. In this case, only the term $o\left(R^{\frac{p}{2}}\right)$ remains on the right-hand side of the asymptotics in Theorem 1.6. On the other hand, if $p$ is even, then $\mathcal{P P} \neq \emptyset$ and $\mu_{p}>0$. In this latter case, the leading terms in the asymptotics of Theorem 1.6 are of order $R^{\frac{p}{2}}$ for generic test-functions, see Proposition 1.11 below. Note however that we obtain $o\left(R^{\frac{p}{2}}\right)$ if, for example, the $\left(\phi_{i}\right)_{1 \leqslant i \leqslant p}$ are pairwise orthogonal for the $L^{2}$ inner product defined by the Lebesgue measure.

In order to interpret this result, let us describe the expectation and the covariance structure of the linear statistics of $\nu_{R}$. First, we describe the expectation of $\nu_{R}$ for any fixed $R>0$. Note that Proposition 1.8 below is a natural extension of the results of Kac [23] and Rice [33], who computed the expectation of Card $(Z \cap[0, R])$. Recall that a Radon measure is a continuous linear form on $\left(\mathcal{C}_{c}^{0}(\mathbb{R}),\|\cdot\|_{\infty}\right)$, the space of compactly supported continuous functions equipped with the sup-norm.

Proposition 1.8 (Expectation of the linear statistics). Let $f$ be a normalized stationary centered Gaussian $\mathcal{C}^{1}$-process. Let $R>0$ and let $\nu_{R}$ denote the counting measure of $\{x \in \mathbb{R} \mid f(R x)=0\}$. Let $\phi: \mathbb{R} \rightarrow \mathbb{R}$ be a Borel-measurable function. If $\phi$ is non-negative or Lebesgue-integrable then,

$$
\mathbb{E}\left[\left\langle\nu_{R}, \phi\right\rangle\right]=\frac{R}{\pi} \int_{-\infty}^{+\infty} \phi(x) \mathrm{d} x .
$$

In particular, as Radon measures $\mathbb{E}\left[\nu_{R}\right]=\frac{R}{\pi} \mathrm{d} x$, where $\mathrm{d} x$ stands for the Lebesgue measure of $\mathbb{R}$.

Remark 1.9. If $\phi: \mathbb{R} \rightarrow \mathbb{R}$ is Lebesgue-integrable we can apply Proposition 1.8 to $|\phi|$. This proves that, for all $R>0$, almost surely $\left\langle\nu_{R},|\phi|\right\rangle\langle+\infty$. Thus, the random variable $\left\langle\nu_{R}, \phi\right\rangle$ is almost surely well-defined. Moreover $\left|\left\langle\nu_{R}, \phi\right\rangle\right| \leqslant\left\langle\nu_{R},|\phi|\right\rangle$, so that $\left\langle\nu_{R}, \phi\right\rangle$ is an integrable random variable, and it makes sense to consider its expectation.

Remark 1.10. In this paper, we consider quantities of the form $\langle\nu, \phi\rangle=\sum_{x \in Z} \phi(x)$, where $Z$ is discrete. If $\phi$ is only defined up to modifications on a negligible set, these quantities are ill-defined. However, let $\nu_{R}$ be is as in Proposition 1.8 and let $\phi_{1}$ and $\phi_{2}$ be test-functions such that $\phi_{1}=\phi_{2}$ almost everywhere. By Proposition 1.8, we have $\mathbb{E}\left[\left\langle\nu_{R},\left|\phi_{1}-\phi_{2}\right|\right\rangle\right]=0$, so that $\left\langle\nu_{R},\left|\phi_{1}-\phi_{2}\right|\right\rangle=0$ almost surely. This implies $\left\langle\nu_{R}, \phi_{1}\right\rangle=\left\langle\nu_{R}, \phi_{2}\right\rangle$ almost surely, since $\left|\left\langle\nu_{R}, \phi_{1}\right\rangle-\left\langle\nu_{R}, \phi_{2}\right\rangle\right| \leqslant\left\langle\nu_{R},\left|\phi_{1}-\phi_{2}\right|\right\rangle$.

The following result gives the large $R$ asymptotics of the covariance of $\left\langle\nu_{R}, \phi_{1}\right\rangle$ and $\left\langle\nu_{R}, \phi_{2}\right\rangle$, where $\phi_{1}$ and $\phi_{2}$ are test-functions (see Definition 1.2). To the best of our knowledge, this result was only known for $\phi_{1}=\phi_{2}=\mathbb{1}_{[0,1]}$ until now, see [15, Theorem 1]. Note that the positivity of the leading constant $\sigma$ in not proved in [15].

Proposition 1.11 (Covariances asymptotics). Let $f$ be a normalized stationary centered Gaussian $\mathcal{C}^{2}$-process and let $\kappa$ denote its correlation function. We assume that $\kappa$ and $\kappa^{\prime \prime}$ are square-integrable and that $\|\kappa\|_{2, \eta}$ tends to 0 as $\eta \rightarrow+\infty$.

Then there exists $\sigma>0$ such that, for any test-functions $\phi_{1}$ and $\phi_{2}$ we have:

$$
m_{2}\left(\nu_{R}\right)\left(\phi_{1}, \phi_{2}\right)=R \sigma^{2} \int_{-\infty}^{+\infty} \phi_{1}(x) \phi_{2}(x) \mathrm{d} x+o(R)
$$


as $R \rightarrow+\infty$. Moreover, we have:

$$
\sigma^{2}=\frac{1}{\pi}+\frac{2}{\pi^{2}} \int_{0}^{+\infty}\left(\frac{1-\kappa(t)^{2}-\kappa^{\prime}(t)^{2}}{\left(1-\kappa(t)^{2}\right)^{\frac{3}{2}}}\left(\sqrt{1-a(t)^{2}}+a(t) \arcsin (a(t))\right)-1\right) \mathrm{d} t,
$$

where $a:(0,+\infty) \rightarrow[-1,1]$ is the map defined by $a: t \mapsto \frac{\kappa(t) \kappa^{\prime}(t)^{2}-\kappa(t)^{2} \kappa^{\prime \prime}(t)+\kappa^{\prime \prime}(t)}{1-\kappa(t)^{2}-\kappa^{\prime}(t)^{2}}$.

Remark 1.12. The fact that $\sigma>0$ is non-trivial. It is proved in Section 4.2 , using the Wiener-Itô expansion of Card $(Z \cap[0, R])$ derived in [26]. In Corollary 4.8, we obtain the following explicit lower bound:

$$
\sigma^{2} \geqslant \frac{1}{\pi^{2}} \int_{0}^{+\infty}\left(\kappa(z)+\kappa^{\prime \prime}(z)\right)^{2} \mathrm{~d} z>0
$$

If we consider the Bargmann-Fock process $f_{B F}$, that is if $\kappa: x \mapsto e^{-\frac{1}{2} x^{2}}$, the previous lower bound gives $\sigma_{B F}^{2} \geqslant\left(2 \pi^{3}\right)^{-\frac{1}{2}} \simeq 0.12 \ldots$ In [17, Proposition 3.1 and Remark 1], Dalmao computed $\sigma_{B F}^{2}$ up to a factor $\frac{1}{\pi}$. Using his result, we get $\sigma_{B F}^{2} \simeq 0,18 \ldots$ Note that these values are smaller than $\frac{1}{\pi}$, hence the integral on the right-hand side of Equation (1.2) is negative.

\subsection{Clustering for the $k$-point functions}

Let $p \geqslant 2$ be an integer and let $f$ be as above a normalized centered stationary Gaussian process. The first step in the proof of our moments asymptotics (Theorem 1.6) is to derive a tractable integral expression of the central moments $m_{p}\left(\nu_{R}\right)\left(\phi_{1}, \ldots, \phi_{p}\right)$ that we want to estimate. Using the Kac-Rice formula (see Proposition 3.6), we write $m_{p}\left(\nu_{R}\right)\left(\phi_{1}, \ldots, \phi_{p}\right)$ as a linear combination of terms of the form

$$
\int_{\mathbb{R}^{k}} \Phi_{R}(x) \rho_{k}(x) \mathrm{d} x,
$$

where $1 \leqslant k \leqslant p$ and $\Phi: \mathbb{R}^{k} \rightarrow \mathbb{R}$ is an integrable function built from the $\left(\phi_{i}\right)_{1 \leqslant i \leqslant p}$. In this equation, the function $\rho_{k}$ is the Kac-Rice density of order $k$ (cf. Definition 3.1). In order to give some meaning to this density, notice that it coincides with the $k$-point function of the random point process $Z=f^{-1}(0)$, see Lemma 3.11. By this we mean that, for any $x=\left(x_{i}\right)_{1 \leqslant i \leqslant k} \in \mathbb{R}^{k}$ such that $\rho_{k}(x)$ is well-defined, we have:

$$
\frac{1}{(2 \varepsilon)^{k}} \mathbb{E}\left[\prod_{i=1}^{k} \operatorname{Card}\left(Z \cap\left[x_{i}-\varepsilon, x_{i}+\varepsilon\right]\right)\right] \underset{\varepsilon \rightarrow 0}{\longrightarrow} \rho_{k}(x) .
$$

The core of the proof of Theorem 1.6 is to understand the large $R$ asymptotics of integrals of the form (1.3). This leads to a detailed study of the Kac-Rice densities $\left(\rho_{k}\right)_{k \in \mathbb{N}^{*}}$. Given $k \in \mathbb{N}^{*}$, Definition 3.1 allows to define $\rho_{k}(x)$ for any $x=\left(x_{i}\right)_{1 \leqslant i \leqslant k}$ such that the Gaussian vector $\left(f\left(x_{i}\right)\right)_{1 \leqslant i \leqslant k}$ is non-degenerate. In particular, if the correlation function $\kappa$ of $f$ tends to 0 at infinity, as in Theorem 1.6 and Proposition 1.11, the ergodicity of $f$ implies that $\rho_{k}$ is well-defined on $\mathbb{R}^{k} \backslash \Delta_{k}$, where we denoted by $\Delta_{k}=\left\{\left(x_{1}, \ldots, x_{k}\right) \in \mathbb{R}^{k} \mid \exists i, j \in\{1, \ldots, k\}, i \neq j\right.$ and $\left.x_{i}=x_{j}\right\}$ the large diagonal in $\mathbb{R}^{k}$ (cf. Lemma 2.10 for more details). In general, $\rho_{k}$ is a continuous symmetric function defined on some symmetric open subset of $\mathbb{R}^{k} \backslash \Delta_{k}$, see Lemmas 3.5 and 3.9.

Interpreting $\rho_{k}$ as the $k$-point function of $Z$, some of the intermediate results in the proof of Theorem 1.6 appear to be of independent interest. Theorems 1.13 and 1.14 below are analogous to the main results of [30], where Nazarov and Sodin studied the $k$-point function of a Gaussian Entire Function. Note however that our methods are completely different. In particular, we do not require any form of analyticity. 
Theorem 1.13 (Vanishing order of the $k$-point function). Let $k \in \mathbb{N}^{*}$, let $f$ be a normalized stationary centered Gaussian $\mathcal{C}^{k}$-process. Let $y=\left(y_{i}\right)_{1 \leqslant i \leqslant k} \in \mathbb{R}^{k}$ and let $\mathcal{I} \in \mathcal{P}_{k}$ be the partition defined by:

$$
\forall i, j \in\{1, \ldots, k\}, \quad y_{i}=y_{j} \Longleftrightarrow \exists I \in \mathcal{I},\{i, j\} \subset I .
$$

For any $I \in \mathcal{I}$, we denote by $|I|$ the cardinality of $I$ and by $y_{I} \in \mathbb{R}$ the common value of the $\left(y_{i}\right)_{i \in I}$. Let us assume that the Gaussian vector $\left(f^{(i)}\left(y_{I}\right)\right)_{I \in \mathcal{I}, 0 \leqslant i<|I|}$ is non-degenerate and denote by

$$
\ell(y)=\left(\prod_{I \in \mathcal{I}} \prod_{i=0}^{|I|-1} \frac{i !}{|I| !}\right) \frac{\mathbb{E}\left[\prod_{I \in \mathcal{I}}\left|f^{(|I|)}\left(y_{I}\right)\right|^{|I|} \mid \forall I \in \mathcal{I}, \forall i \in\{0, \ldots,|I|-1\}, f^{(i)}\left(y_{I}\right)=0\right]}{(2 \pi)^{\frac{k}{2}} \operatorname{det}\left(\operatorname{Var}\left(\left(f^{(i)}\left(y_{I}\right)\right)_{I \in \mathcal{I}, 0 \leqslant i<|I|}\right)\right)^{\frac{1}{2}}}
$$

where $\mathbb{E}\left[\prod_{I \in \mathcal{I}}\left|f^{(|I|)}\left(y_{I}\right)\right|^{|I|} \mid \forall I \in \mathcal{I}, \forall i \in\{0, \ldots,|I|-1\}, f^{(i)}\left(y_{I}\right)=0\right]$ stands for the conditional expectation of $\prod_{I \in \mathcal{I}}\left|f^{(|I|)}\left(y_{I}\right)\right|^{|I|}$ given that $f^{(i)}\left(y_{I}\right)=0$ for all $I \in \mathcal{I}$ and $i \in\{0, \ldots,|I|-1\}$.

Then, there exists a neighborhood $U$ of $y$ in $\mathbb{R}^{k}$ such that the $k$-point function $\rho_{k}$ of $f^{-1}(0)$ is well-defined on $U \backslash \Delta_{k}$ and, as $x \rightarrow y$ with $x=\left(x_{i}\right)_{1 \leqslant i \leqslant k} \in U \backslash \Delta_{k}$, we have:

$$
\left(\prod_{I \in \mathcal{I}} \prod_{\left\{(i, j) \in I^{2} \mid i<j\right\}} \frac{1}{\left|x_{i}-x_{j}\right|}\right) \rho_{k}(x) \underset{x \rightarrow y}{\longrightarrow} \ell(y) .
$$

Moreover, if $\left(f^{(i)}\left(y_{I}\right)\right)_{I \in \mathcal{I}, 0 \leqslant i \leqslant|I|}$ is non-degenerate, then $\ell(y)>0$.

If the process $f$ is of class $\mathcal{C}^{k}$ and such that $\kappa(x) \underset{x \rightarrow+\infty}{\longrightarrow} 0$, then the non-degeneracy conditions in Theorem 1.13 are satisfied for all $y \in \mathbb{R}^{k}$ (cf. Lemma 2.10 below). In this case, $\rho_{k}$ is well-defined on $\mathbb{R}^{k} \backslash \Delta_{k}$, and $\ell(y)$ is positive for any $y \in \mathbb{R}^{k}$. If $y \in \mathbb{R}^{k} \backslash \Delta_{k}$, the partition associated with $y$ is $\mathcal{I}=\{\{i\} \mid 1 \leqslant i \leqslant k\} \in \mathcal{P}_{k}$. Then, $\ell(y)=\rho_{k}(y)$ (see Equation (1.4) and Definition 3.1) and Theorem 1.13 only states that $\rho_{k}$ is continuous at $y$ and that $\rho_{k}(y)>0$. If $y \in \Delta_{k}$, Theorem 1.13 shows that $\rho_{k}(x) \underset{x \rightarrow y}{\longrightarrow} 0$. In particular, under the assumption that $\kappa$ tends to 0 at infinity, the $k$-point function of $Z$ can be uniquely extended into a continuous function on $\mathbb{R}^{k}$ that vanishes exactly on $\Delta_{k}$. In this case, the last part of the theorem gives the vanishing order of $\rho_{k}$ near any point of the diagonal. The fact that $\rho_{k}$ vanishes along $\Delta_{k}$ is interpreted as the sign of a short-range repulsion between zeros of $f$. The estimates of Theorem 1.13 quantify this phenomenon.

Let us now consider the long-range correlations between zeros of $f$. We still concern ourselves with the case where $\kappa$ tends to 0 at infinity. Let $A$ and $B$ be two non-empty disjoint intervals of $\mathbb{R}$. If $A$ and $B$ are far enough from one another, the values of $f$ on $A$ are essentially uncorrelated with those of $f$ on $B$. It is then reasonable to expect the point processes $Z \cap A$ and $Z \cap B$ to be roughly independent. The independence of $Z \cap A$ and $Z \cap B$ would imply that $\rho_{k+l}(x, y)=\rho_{k}(x) \rho_{l}(y)$ for any $k, l \in \mathbb{N}^{*}$, any $x \in A^{k} \backslash \Delta_{k}$ and any $y \in B^{l} \backslash \Delta_{l}$. The following result shows that a relation of this form holds, up to an error term.

Theorem 1.14 (Clustering for $k$-point functions). Let $k \in \mathbb{N}^{*}$, let $f$ be a normalized stationary centered Gaussian $\mathcal{C}^{k}$-process whose correlation function $\kappa$ is such that $\|\kappa\|_{k, \eta} \underset{\eta \rightarrow+\infty}{\longrightarrow} 0$. For any $l \in\{1, \ldots, k\}$, let $\rho_{l}$ denote the $l$-point function of $f^{-1}(0)$.

Then, there exists $C>0$ such that for all $x=\left(x_{i}\right)_{1 \leqslant i \leqslant k} \in \mathbb{R}^{k} \backslash \Delta_{k}$ we have:

$$
0 \leqslant \rho_{k}(x) \leqslant C \prod_{1 \leqslant i<j \leqslant k} \min \left(\left|x_{i}-x_{j}\right|, 1\right) .
$$


Moreover, for all $\eta \geqslant 1$, for all $\mathcal{I} \in \mathcal{P}_{k}$, for all $x=\left(x_{i}\right)_{1 \leqslant i \leqslant k} \in \mathbb{R}^{k} \backslash \Delta_{k}$ satisfying:

$$
\forall I, J \in \mathcal{I} \text { such that } I \neq J, \forall i \in I, \forall j \in J,\left|x_{i}-x_{j}\right|>\eta,
$$

we have:

$$
\prod_{I \in \mathcal{I}} \rho_{|I|}\left(\underline{x}_{I}\right)=\rho_{k}(x)\left(1+O\left(\left(\|\kappa\|_{k, \eta}\right)^{\frac{1}{2}}\right)\right)
$$

where the constant involved in the error term $O\left(\left(\|\kappa\|_{k, \eta}\right)^{\frac{1}{2}}\right)$ does not depend on $\eta, \mathcal{I}$ nor $x$. Here, we denoted by $|I|$ the cardinality of $I$ and by $\underline{x}_{I}=\left(x_{i}\right)_{i \in I}$, for all $I \in \mathcal{I}$.

An important new idea in the proof of Theorems 1.13 and 1.14 is that we derive a whole family of new expressions for the $k$-point functions $\rho_{k}$, indexed by the partitions of $\{1, \ldots, k\}$. For any point $x \in \mathbb{R}^{k}$, at least one of these expressions is uniformly nondegenerate in a neighborhood of $x$. Then, studying $\rho_{k}$ is mostly a matter of choosing the right expression, depending on the domain we are considering. These new expressions are introduced and studied in Section 6, using the divided differences introduced in Section 5. A key idea is that divided differences allow to replace the random vector $\left(f\left(x_{i}\right)\right)_{1 \leqslant i \leqslant k}$, appearing the original expression of $\rho_{k}\left(x_{1}, \ldots, x_{k}\right)$, by another Gaussian vector which is never degenerate even on the diagonal. We will discuss these ideas further in Section 1.5 below.

\subsection{Law of large numbers and central limit theorem}

As an application of the moments estimates of Theorem 1.6, we prove a strong Law of Large Numbers and a Central Limit Theorem. These theorems hold in the large $R$ limit, for the linear statistics $\left\langle\nu_{R}, \phi\right\rangle$ with $\phi$ a test-function (cf. Definition 1.2), but also for the random measures $\nu_{R}$.

Remark 1.15. Under the hypotheses of Proposition 1.11, we immediately obtain a weak Law of Large Number for the linear statistics by applying Markov's Inequality and using the variance estimates of Proposition 1.11. That is, for any test-function $\phi$, for all $\varepsilon>0$, we have:

$$
\mathbb{P}\left(\left|\frac{1}{R}\left\langle\nu_{R}, \phi\right\rangle-\frac{1}{\pi} \int_{\mathbb{R}} \phi(x) \mathrm{d} x\right|>\varepsilon\right)=O\left(R^{-1}\right) .
$$

In fact, if the correlation function $\kappa$ of $f$ decays fast enough, we can combine the moments estimates of Theorem 1.6 with Markov's Inequality and the Borel-Cantelli Lemma to obtain the following.

Theorem 1.16 (Strong Law of Large Numbers). Let $p \in \mathbb{N}^{*}$ and $f$ be a normalized stationary centered Gaussian $\mathcal{C}^{2 p}$-process whose correlation function $\kappa$ is such that $\|\kappa\|_{2 p, \eta}=o\left(\eta^{-8 p}\right)$ as $\eta \rightarrow+\infty$. Let $\left(R_{n}\right)_{n \in \mathbb{N}}$ be a sequence of positive numbers that satisfies $\sum_{n \in \mathbb{N}} R_{n}^{-p}<+\infty$.

Then, for any test-function $\phi$, the following holds almost surely:

$$
\frac{1}{R_{n}}\left\langle\nu_{R_{n}}, \phi\right\rangle \underset{n \rightarrow+\infty}{\longrightarrow} \frac{1}{\pi} \int_{\mathbb{R}} \phi(x) \mathrm{d} x .
$$

Moreover, we have $\frac{1}{R_{n}} \nu_{R_{n}} \underset{n \rightarrow+\infty}{\longrightarrow} \frac{1}{\pi} \mathrm{d} x$ almost surely in the weak-* sense.

Let us now recall some classical definitions before stating our Central Limit Theorem.

Notation 1.17 (Gaussian distributions). Let $n \geqslant 1$ and let $\Lambda$ be a positive semi-definite square matrix of size $n$. We denote by $\mathcal{N}(0, \Lambda)$ the centered Gaussian distribution of variance $\Lambda$ in $\mathbb{R}^{n}$. We denote by $X \sim \mathcal{N}(0, \Lambda)$ the fact that distribution of the random vector $X \in \mathbb{R}^{n}$ is $\mathcal{N}(0, \Lambda)$. 
Definition 1.18 (Schwartz space). A function $\phi: \mathbb{R} \rightarrow \mathbb{R}$ is said to be fast-decreasing if it satisfies $\phi(x)=O\left(|x|^{-k}\right)$ as $|x| \rightarrow+\infty$, for all $k \in \mathbb{N}$. The Schwartz space $\mathcal{S}(\mathbb{R})$ is the space of $\mathcal{C}^{\infty}$ functions $\phi$ such that $\phi$ and all its derivatives are fast-decreasing. Finally, we denote by $\mathcal{S}^{\prime}(\mathbb{R})$ the space of tempered generalized functions.

Remark 1.19. - In this paper, we use the terminology "generalized function" instead of "distribution" to avoid any possible confusion with the distribution of a random variable.

- Recall that $\mathcal{S}^{\prime}(\mathbb{R})$ is indeed the topological dual of $\mathcal{S}(\mathbb{R})$, for some topology that we do not recall here.

- We refer to [8] for details about the definition of random elements of $\mathcal{S}^{\prime}(\mathbb{R})$ and the notion of convergence in distribution in this space.

Definition 1.20 (White Noise). The standard Gaussian White Noise $W$ is a random element of $\mathcal{S}^{\prime}(\mathbb{R})$ whose distribution is characterized by:

$$
\forall \phi \in \mathcal{S}(\mathbb{R}), \quad\langle W, \phi\rangle \sim \mathcal{N}\left(0,\|\phi\|_{L^{2}}^{2}\right)
$$

where $\langle\cdot, \cdot\rangle\rangle$ is the canonical pairing between $\mathcal{S}^{\prime}(\mathbb{R})$ and $\mathcal{S}(\mathbb{R})$, and $\|\phi\|_{L^{2}}=\left(\int_{\mathbb{R}} \phi(x)^{2} \mathrm{~d} x\right)^{\frac{1}{2}}$ is the $L^{2}$-norm of $\phi$.

Theorem 1.21 (Central Limit Theorem). Let $f$ be a normalized stationary centered Gaussian process of class $\mathcal{C}^{\infty}$ and let us assume that its correlation function satisfies $\kappa \in \mathcal{S}(\mathbb{R})$. Let $\sigma>0$ be the constant defined by Equation (1.2).

For any test-function $\phi$ (in the sense of Definition 1.2), we have the following convergence in distribution:

$$
\frac{1}{R^{\frac{1}{2}} \sigma}\left(\left\langle\nu_{R}, \phi\right\rangle-\frac{R}{\pi} \int_{\mathbb{R}} \phi(x) \mathrm{d} x\right) \underset{R \rightarrow+\infty}{\longrightarrow} \mathcal{N}\left(0,\|\phi\|_{L^{2}}^{2}\right) .
$$

Moreover, the following holds in distribution in $\mathcal{S}^{\prime}(\mathbb{R})$ :

$$
\frac{1}{R^{\frac{1}{2}} \sigma}\left(\nu_{R}-\frac{R}{\pi} \mathrm{d} x\right) \underset{R \rightarrow+\infty}{\longrightarrow} W
$$

where $W$ is the standard Gaussian White Noise and $\mathrm{d} x$ is the Lebesgue measure of $\mathbb{R}$.

The fact that almost surely $\nu_{R} \in \mathcal{S}^{\prime}(\mathbb{R})$ for all $R>0$ is not obvious. This is proved in Lemma 3.12 as a consequence of Proposition 1.8, see Section 3.3.

\subsection{Sketch of proof}

In this section, we discuss the main ideas of the proofs of our main results (Theorems 1.6, 1.13 and 1.14). First, let us outline the proof of Theorem 1.6 assuming the results of Theorem 1.14. The starting point of the proof is the Kac-Rice formula, see Proposition 3.6 below. It allows to write the non-central moments of the linear statistics associated with the random measure $\nu_{R}$ as follows:

$$
\mathbb{E}\left[\prod_{i=1}^{k}\left\langle\nu_{R}, \phi_{i}\right\rangle\right]=\int_{\mathbb{R}^{k}}\left(\prod_{i=1}^{k} \phi_{i}\left(\frac{x_{i}}{R}\right)\right) \rho_{k}\left(x_{1}, \ldots, x_{k}\right) \mathrm{d} x_{1} \ldots \mathrm{d} x_{k},
$$

where $\left(\phi_{i}\right)_{1 \leqslant i \leqslant k}$ are test-functions satisfying the hypotheses of Theorem 1.6 and $\rho_{k}$ is the function defined by Definition 3.1 below. Here we are cheating a bit: Equation (1.5) is false and the $k$-th non-central moment on the left-hand side should be replaced be the so-called $k$-th factorial moment for this relation to hold. However, the $k$-th non-central moment can be expressed in terms of the factorial moments of order at most $k$ by some 
combinatorics, so that a more complicated version of Equation (1.5) holds. Dealing with these combinatorics is one of the difficulties of the proof of Theorem 1.6 given in Section 7. For the sake of clarity, in this sketch of proof we will not give more details about this, and simply pretend that Equation (1.5) holds. This is enough to understand the main ideas of the proof.

Under the hypotheses of Theorem 1.6, for any $k \in\{1, \ldots, p\}$ the density $\rho_{k}$ is welldefined from $\mathbb{R}^{k} \backslash \Delta_{k}$ to $\mathbb{R}$, but it is a priori singular along $\Delta_{k}$. As discussed in Section 1.3, the Kac-Rice density $\rho_{k}$ is equal to the $k$-point function of the zero point process of $f$. By the first point in Theorem 1.14, it admits a unique continuous extension to $\mathbb{R}^{k}$ which is bounded. In particular, the right-hand side of Equation (1.5) is well-defined and finite. Let $A \subset\{1, \ldots, p\}$, we denote by $|A|$ its cardinality. Moreover, for any $x=\left(x_{i}\right)_{1 \leqslant i \leqslant p} \in \mathbb{R}^{p}$, we denote by $\underline{x}_{A}=\left(x_{i}\right)_{i \in A}$. Then, using Equation (1.5), we can write $m_{p}\left(\nu_{R}\right)\left(\phi_{1}, \ldots, \phi_{p}\right)$ as:

$$
m_{p}\left(\nu_{R}\right)\left(\phi_{1}, \ldots, \phi_{p}\right)=\int_{\mathbb{R}^{p}}\left(\prod_{i=1}^{p} \phi_{i}\left(\frac{x_{i}}{R}\right)\right) F_{p}(x) \mathrm{d} x,
$$

where,

$$
F_{p}: x \longmapsto \sum_{A \subset\{1, \ldots, p\}}(-1)^{p-|A|} \rho_{|A|}\left(\underline{x}_{A}\right) \prod_{i \notin A} \rho_{1}\left(x_{i}\right) .
$$

See Lemma 7.3 for the rigorous statement corresponding to Equation (1.6). Note that we only use the notation $F_{p}$ in the present section. In Section 7 , this function is the one denoted by $F_{\mathcal{I}_{\min }(p)}$.

Apart from proving Theorem 1.14, the main difficulty in the proof of our moments estimates is to understand the large $R$ asymptotics of the integral appearing in Equation (1.6). In order to do so, we cut $\mathbb{R}^{p}$ into pieces as follows. Let $\eta \geqslant 0$, for any $x=\left(x_{i}\right)_{1 \leqslant i \leqslant p} \in \mathbb{R}^{p}$, we denote by $G_{\eta}(x)$ the graph whose set of vertices is $\{1, \ldots, p\}$ and such that there is an edge between $i$ and $j$ if and only if $i \neq j$ and $\left|x_{i}-x_{j}\right| \leqslant \eta$. We denote by $\mathcal{I}_{\eta}(x) \in \mathcal{P}_{p}$ the partition defined by the connected components of $G_{\eta}(x)$. This partition encodes how the components of $x$ are clustered in $\mathbb{R}$, at scale $\eta$. Finally, for any $\mathcal{I} \in \mathcal{P}_{p}$, we denote by $\mathbb{R}_{\mathcal{I}, \eta}^{p}=\left\{x \in \mathbb{R}^{p} \mid \mathcal{I}_{\eta}(x)=\mathcal{I}\right\}$. We have $\mathbb{R}^{p}=\bigsqcup_{\mathcal{I} \in \mathcal{P}_{p}} \mathbb{R}_{\mathcal{I}, \eta}^{p}$, so that it is enough to understand the contribution of each $\mathbb{R}_{\mathcal{I}, \eta}^{p}$ to the integral appearing in Equation (1.6).

Since we are interested in the asymptotics as $R \rightarrow+\infty$, we choose a scale parameter $\eta(R)$ that depends on $R$. The most convenient choice for $\eta$ is the following. Under the hypotheses of Theorem 1.6, there exists a function $\alpha$ such that, setting $\eta: R \mapsto R^{\frac{1}{4}} \alpha(R)$, we have the following as $R \rightarrow+\infty: \eta(R) \rightarrow+\infty, \alpha(R) \rightarrow 0$ and $\|\kappa\|_{p, \eta(R)}=o\left(R^{-p}\right)$. In particular, the error term in Theorem 1.14 becomes $o\left(R^{-\frac{p}{2}}\right)$. Then, the contribution of $\mathbb{R}_{\mathcal{I}, \eta(R)}^{p}$ to (1.6) depends on the combinatorics of $\mathcal{I}$, and one of the following three situations occurs.

1. The partition $\mathcal{I}$ contains a singleton, say $\{p\} \in \mathcal{I}$. This means that if $x \in \mathbb{R}_{\mathcal{I}, \eta(R)}^{p}$, then $x_{p}$ is far from the other components of $x$, at scale $\eta(R)$. In this case, for each $A \subset\{1, \ldots, p-1\}$, we regroup the terms indexed by $A$ and $A \sqcup\{p\}$ in Equation (1.7). Using the clustering property of Theorem 1.14, these two terms cancel each other out, up to an error term of order $o\left(R^{-\frac{p}{2}}\right)$. Summing over $A \subset\{1, \ldots, p-1\}$, we obtain $F_{p}(x)=o\left(R^{-\frac{p}{2}}\right)$ uniformly on $\mathbb{R}_{\mathcal{I}, \eta(R)}^{p}$. This implies that $\mathbb{R}_{\mathcal{I}, \eta(R)}^{p}$ only contributes $o\left(R^{\frac{p}{2}}\right)$ to (1.6).

2. If $\mathcal{I}$ does not contain any singletons, we denote by $a$ the number of pairs in $\mathcal{I}$ and by $b$ the number of elements of $\mathcal{I}$ of cardinality at least 3 . In the second situation we consider, we assume that $b \geqslant 1$. In this case, we prove that the contribution of $\mathbb{R}_{\mathcal{I}, \eta(R)}^{p}$ to (1.6) is $O\left(R^{a+b} \eta(R)^{p-2 a-b}\right)$. This bound is obtained by using the 
clustering property of Theorem 1.14 in a way similar to what we did in the previous case. The dissymmetry between the pairs and the other elements of $\mathcal{I}$ comes from the integrability of the function $z \mapsto F_{2}(0, z)$ on $\mathbb{R}$. This dissymmetry is crucial in the following. Using the relation $\eta(R)=R^{\frac{1}{4}} \alpha(R)$ and $2 a+3 b \leqslant p$, we have:

$$
R^{a+b} \eta(R)^{p-2 a-b}=O\left(R^{\frac{p}{2}} \alpha(R)^{p-2 a-b}\right) .
$$

Since $b \geqslant 1$, we have $2 a+b<p$ and the previous term is $o\left(R^{\frac{p}{2}}\right)$. Once again, $\mathbb{R}_{\mathcal{I}, \eta(R)}^{p}$ only contributes $o\left(R^{\frac{p}{2}}\right)$ to (1.6).

3. The last situation is when $\mathcal{I}=\left\{\left\{a_{i}, b_{i}\right\} \mid 1 \leqslant i \leqslant \frac{p}{2}\right\}$ is a partition into pairs, which can only happen if $p$ is even. In this case, the clustering property of Theorem 1.14 implies that $F_{p}(x)=\prod_{i=1}^{\frac{p}{2}} F_{2}\left(x_{a_{i}}, x_{b_{i}}\right)+o\left(R^{-\frac{p}{2}}\right)$, uniformly on $\mathbb{R}_{\mathcal{I}, \eta(R)}^{p}$. This implies that the contribution of $\mathbb{R}_{\mathcal{I}, \eta(R)}^{p}$ to (1.6) equals:

$$
\prod_{i=1}^{\frac{p}{2}} \int_{\mathbb{R}^{2}} \phi_{a_{i}}\left(\frac{x}{R}\right) \phi_{b_{i}}\left(\frac{y}{R}\right) F_{2}(x, y) \mathrm{d} x \mathrm{~d} y+o\left(R^{\frac{p}{2}}\right)=\prod_{i=1}^{\frac{p}{2}} m_{2}\left(\nu_{R}\right)\left(\phi_{a_{i}}, \phi_{b_{i}}\right)+o\left(R^{\frac{p}{2}}\right)
$$

We conclude the proof of Theorem 1.6 by summing up over $\mathcal{I} \in \mathcal{P}_{p}$ the contributions of each $\mathbb{R}_{\mathcal{I}, \eta(R)}^{p}$ to the integral in Equation (1.6). Note that the leading term comes from the pieces indexed by partitions into pairs.

Let us now consider the proofs of Theorems 1.13 and 1.14. In this sketch of proof, we assume that the correlation function $\kappa$ of $f$ tends to 0 at infinity. This ensures that $\rho_{k}$ is well-defined on $\mathbb{R}^{k} \backslash \Delta_{k}$. By Definition 3.1, for any $x=\left(x_{i}\right)_{1 \leqslant i \leqslant k} \in \mathbb{R}^{k} \backslash \Delta_{k}$ we have $\rho_{k}(x)=(2 \pi)^{-\frac{k}{2}} N_{k}(x) D_{k}(x)^{-\frac{1}{2}}$, where $D_{k}(x)$ is the determinant of the variance matrix of $\left(f\left(x_{1}\right), \ldots, f\left(x_{k}\right)\right)$ and $N_{k}(x)$ is the conditional expectation of $\prod_{i=1}^{k}\left|f^{\prime}\left(x_{i}\right)\right|$ given that $f\left(x_{1}\right)=\cdots=f\left(x_{k}\right)=0$. The density $\rho_{k}$ is a priori singular along the large diagonal $\Delta_{k} \subset \mathbb{R}^{k}$, since $D_{k}$ vanishes along $\Delta_{k}$. The main problem here is to understand to behavior of $\rho_{k}$, that is of $N_{k}$ and $D_{k}$, near $\Delta_{k}$. This is what we focus on in the remainder of this section. Once this is done, the clustering result of Theorem 1.14 is a (non-trivial) consequence of the decay at infinity of $\kappa$ and its derivatives.

Our study of $N_{k}$ and $D_{k}$ near $\Delta_{k}$ relies on the use of the divided differences associated with the process $f$. Let us explain our strategy on the simplest non-trivial case, that is for $D_{2}$. A direct computation, using the Taylor expansion of $\kappa$ around 0 , shows that, in the setting of this paper, we have $D_{2}(x, y) \sim(y-x)^{2}$ as $y \rightarrow x$. This proof is very simple, but its extension to 3 points or more seems intractable. Here is another proof of the same result that can be generalized to $k \geqslant 3$. If $y \neq x$, we can write:

$$
\left(\begin{array}{l}
f(x) \\
f(y)
\end{array}\right)=\left(\begin{array}{cc}
1 & 0 \\
1 & y-x
\end{array}\right)\left(\begin{array}{c}
f(x) \\
\frac{f(y)-f(x)}{y-x}
\end{array}\right)
$$

As $y \rightarrow x$, we have $\left(f(x), \frac{f(y)-f(x)}{y-x}\right) \rightarrow\left(f(x), f^{\prime}(x)\right)$. By stationarity and normalization of $f$, the matrix $\operatorname{Var}\left(f(x), f^{\prime}(x)\right)$ is the identity. Hence, taking the determinant of the variance of (1.8), we recover $D_{2}(x, y) \sim(y-x)^{2}$ as $y \rightarrow x$.

In Equation (1.8), by stationarity, normalization and regularity of $f$, the Gaussian vector $\left(f(x), \frac{f(y)-f(x)}{y-x}\right)$ is uniformly non-degenerate in a neighborhood of $\Delta_{2}$. Thus, the degeneracy of $(f(x), f(y))$ along $\Delta_{2}$ is completely accounted for by the degeneracy of the

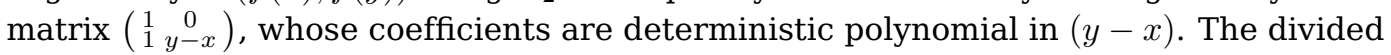
differences generalize this situation to any number of points. By definition of the divided differences $\left([f]_{j}\left(x_{1}, \ldots, x_{j}\right)\right)_{1 \leqslant j \leqslant k}$ associated with $f$ and $x=\left(x_{i}\right)_{1 \leqslant i \leqslant k} \in \mathbb{R}^{k} \backslash \Delta_{k}$ (see 
Section 5.1), we have:

$$
\left(\begin{array}{c}
f\left(x_{1}\right) \\
\vdots \\
f\left(x_{k}\right)
\end{array}\right)=M(x)\left(\begin{array}{c}
{[f]_{1}\left(x_{1}\right)} \\
\vdots \\
{[f]_{k}\left(x_{1}, \ldots, x_{k}\right)}
\end{array}\right)
$$

where the coefficients of the matrix $M(x)$ are deterministic polynomials in $\left(x_{j}-x_{i}\right)_{i<j}$. In fact, $\operatorname{det} M(x)=\prod_{1 \leqslant i<j \leqslant k}\left(x_{j}-x_{i}\right)$ and we have:

$$
\left([f]_{1}\left(x_{1}\right),[f]_{2}\left(x_{1}, x_{2}\right), \ldots,[f]_{k}\left(x_{1}, \ldots, x_{k}\right)\right) \underset{x \rightarrow(z, z, \ldots, z)}{\longrightarrow}\left(f(z), f^{\prime}(z), \ldots, \frac{f^{(k-1)}(z)}{(k-1) !}\right) .
$$

Our hypotheses ensure that the Gaussian vector on the right-hand side is non-degenerate. Denoting by $D>0$ the determinant of its variance, this proves that, as $x \rightarrow(z, z, \ldots, z)$, we have $D_{k}(x) \sim D \prod_{1 \leqslant i<j \leqslant k}\left(x_{j}-x_{i}\right)^{2}$. Note that $D$ does not depend on $z$, by stationarity. A refinement of this argument shows that $N_{k}(x) \sim N \prod_{1 \leqslant i<j \leqslant k}\left(x_{j}-x_{i}\right)^{2}$ for some $N>0$. Hence, as $x \rightarrow(z, z, \ldots, z)$ we have:

$$
\rho_{k}(x) \sim \frac{N}{(2 \pi)^{\frac{k}{2}} D^{\frac{1}{2}}} \prod_{1 \leqslant i<j \leqslant k}\left|x_{j}-x_{i}\right| .
$$

The previous discussion explains how the divided differences allow to understand the apparent singularity of $\rho_{k}$ near $\{(z, z, \ldots, z) \mid z \in \mathbb{R}\} \subset \mathbb{R}^{k}$, which is the stratum of smallest dimension in $\Delta_{k}$. Near other strata, the situation is more intricate, yet tractable by similar methods. The key point is that, using the divided differences associated with $f$, we define a family of alternative expressions of $\rho_{k}$ indexed by the partitions of $\{1, \ldots, k\}$, see Definition 6.14. Then, for any $y \in \mathbb{R}^{k}$, we prove the local estimate of Theorem 1.13 by choosing the right expression of $\rho_{k}$, depending on how the components of $y$ are clustered. Precisely, we use the expression indexed by the partition $\mathcal{I}_{0}(y)$ defined previously, see also Definition 6.2.

\subsection{Related works}

The study of the zeros of a Gaussian process goes back to Kac [23], who obtained a formula for the mean number of roots of some Gaussian polynomials in an interval. This was generalized to other Gaussian processes by Rice [33]. The mean number of zeros in an interval of any continuous stationary Gaussian process was computed by Ylvisaker, see [35]. The proofs of Kac and Rice rely on an integral formula for the mean number of zeros. Extensions of their work lead to what are now called the Kac-Rice formulas. Modern references for these are [2] and [6], but formulas of this kind already appear in [13].

Among other things, Kac-Rice formulas were used to derive conditions for the finiteness of the moments of the number of zeros of Gaussian processes. Geman derived a necessary and sufficient condition for the finiteness of the second moment in [22]. The case of higher moments was studied by Cuzick in $[14,16]$. Note that [16] already uses divided differences in order to obtain criteria for the finiteness of the moments of the number of zeros of a Gaussian process. The results of [16] do not apply to the Bargmann-Fock field, whose correlation function is $z \mapsto e^{-\frac{1}{2} z^{2}}$, and which is one of our main example in this paper (cf. Example A.4). Much more recently, a necessary condition for the finiteness of the moment of order $p$ was derived in [5]. We emphasize that the methods developed in the present paper allow to prove the finiteness of the higher moments of the number of zeros of a Gaussian process in an interval under three simple conditions: stationarity, sufficient regularity of the process, and fast enough decay at 
infinity of the correlation function and its first derivatives. While being easy to state and rather general, these conditions are quite strong and probably far from necessary.

In [15], Cuzick studied the asymptotic variance as $R \rightarrow+\infty$ of the number of zeros of a stationary Gaussian process $f$ in $[0, R]$. He obtained the same asymptotics as in Proposition 1.11 for $\phi_{1}=\mathbb{1}_{[0,1]}=\phi_{2}$, under slightly weaker conditions. However, he did not prove the positivity of the constant $\sigma$ (cf. Equation (1.2)). Assuming that $\sigma>0$, he also derived a Central Limit Theorem for $\operatorname{Card}(Z \cap[0, R])$ as $R \rightarrow+\infty$. Piterbarg proved similar results and the positivity of $\sigma$ under different assumptions, see [32, Theorem 3.5] for example.

In [27], Kratz and Leòn developed a method for proving Central Limit Theorems for level functionals of Gaussian processes. In particular, it should allow to prove Theorem 1.21 under weaker hypotheses than those we gave. The method of [27] is completely different and relies on the Wiener-Itô expansion of the functional under study. The Wiener-Itô expansion of $\operatorname{Card}(Z \cap[0, R])$ was computed in [26]. The same proof should yield the expansion of $\left\langle\nu_{R}, \phi\right\rangle$ for any Lebesgue-integrable $\phi$. The results of KratzLeòn also show that the variance of $\operatorname{Card}(Z \cap[0, R])$ is equivalent to $\sigma^{2} R$ as $R \rightarrow+\infty$, for some $\sigma>0$. In Section 4.2, we use the result of [26] to derive the lower bound on $\sigma^{2}$ mentioned in Remark 1.12. Let us mention that, very recently, Lachièze-Rey [28] proved that:

$$
\liminf _{R \rightarrow+\infty} \frac{1}{R} \operatorname{Var}(\operatorname{Card}(Z \cap[0, R]))>0,
$$

under essentially no hypothesis on the process $f$. This implies the positivity of $\sigma^{2}$ in Proposition 1.11. The present paper partially overlaps with [28] since we obtained independently a similar lower bound for $\sigma^{2}$ by the same method, see [28, Section 4] and Corollary 4.8 below.

The references cited previously are concerned with the number of zeros of $f$ in an interval. More generally, a lot of them consider the number of crossings, or up-crossings, of a level by $f$ in an interval. For an in depth survey of the existing literature on the subject we refer to [25].

A special case of [31, Theorem 1] gives the strong Law of Large Numbers for the number of zeros of a stationary Gaussian process $f$ in $[0, R]$, under weaker assumptions than Theorem 1.16. Nazarov and Sodin also studied the $k$-point functions of the point process defined by the complex zeros of a Gaussian Entire Function, see [30]. Theorems 1.13 and 1.14 are analogous to the main results of [30], but for the real zeros of a stationary Gaussian process. We stress that our techniques are different from those of [30]. In particular, in [30] the authors require the analiticity of the Gaussian process and use techniques from complex analysis, such as the Residue Theorem, whereas we only require our Gaussian fields to be $\mathcal{C}^{k}$ in order to obtain a clustering property of the $k$-point function.

The $k$-point functions $\rho_{k}$ of the real zeros of the Bargmann-Fock process were studied by Do and $\mathrm{Vu}$. In [18, Lemma 9], they proved that the $\rho_{k}$ satisfy a clustering property similar to Theorem 1.14, with an exponentially small error term. They also derived the vanishing order of the $\rho_{k}$ along the diagonal, see [18, Lemma 10]. Their methods build on the work of [30] and also relies on complex analysis. In particular, it is paramount in their work that the Bargmann-Fock is the restriction to $\mathbb{R}$ of a Gaussian Entire Function.

In both [30] and [18], the authors deduce from their clustering result a Central Limit Theorem for the (compactly supported) linear statistics of the point processes they study. Their proofs rely on the cumulants method. This strategy was generalized in [10, Theorem 13], where the authors show that a strong clustering property of the kind of Theorem 1.14, with a fast-decreasing error term, implies a Central Limit Theorem for the compactly supported linear statistics of the underlying point process. Note that one 
can not deduce the moments estimates of Theorem 1.6 from this kind of results, even when the correlation function $\kappa$ lies in $\mathcal{S}(\mathbb{R})$.

Under the hypotheses of Theorem 1.6, Markov's Inequality implies the concentration in probability of $\frac{1}{R} \operatorname{Card}(Z \cap[0, R])$, more generally of the normalized linear statistics, around its mean at polynomial speed in $R$. Under stronger assumptions, in [7], the authors proved a large deviation result for $\frac{1}{R} \operatorname{Card}(Z \cap[0, R])$, that is concentration around the mean at exponential speed in $R$. Their proof relies on the existence of an analytic extension of $f$ to horizontal strips in the complex plane. Note that the Bargmann-Fock process satisfies the hypotheses of [7, Theorem 1.1].

In this paper, we study the zeros of a stationary Gaussian process in an interval of size $R$ as $R \rightarrow+\infty$. In [4], we studied the real zeros of a Gaussian section of the $d$-th tensor power of an ample line bundle over a real algebraic curve, as $d \rightarrow+\infty$. The model of Gaussian section considered in [4] is known as the complex Fubini-Study ensemble and was introduced in [21]. It is the real analogue of the complex model studied by Shiffman-Zelditch in [34] and subsequent papers. The idea to study the random measure associated with the zero set of a Gaussian section already appears in [34]. In [12], the authors study the scaling limit of the $k$-point function of the complex zero set of a random section in their model. They also relate this function with the non-central moments of the linear statistics associated with these complex zeros.

In [4], we derived the large $d$ asymptotics for the central moments of the linear statistics associated with the real zero set of a Gaussian section of degree $d$ in the complex Fubini-Study ensemble. These results are the counterpart of Theorems 1.6, 1.16 and 1.21 in this context. Note that [4, Theorem 1.12] generalizes the variance estimate derived by Letendre-Puchol in [29], in the case where the ambient dimension is 1. Its proof relies on results of Ancona, who proved the counterpart of Theorem 1.14 in [3, Theorems 4.1 and 5.7 and Proposition 4.2]. However, note that Theorems 1.14 and 1.13 are more precise than their counterparts in [3]. For example, [3, Theorem 5.7] says that the $k$-point function $\rho_{k}$ vanishes along the diagonal $\Delta_{k}$, while in Theorem 1.13 we also compute the vanishing order of $\rho_{k}$ along the diagonal $\Delta_{k}$, also giving conditions on the process $f$ for which this vanishing order is sharp. As explained in the last paragraph of Section 1.5, one of the fundamental parts of studying the $k$-point function is finding good expression for $\rho_{k}(x)$, depending on how the components of $x$ are clustered. The expressions used in the present article are different from those used in [3] (one should compare the expression appearing in Definition 6.14 with the one in [3, Proposition 5.21]). The new expressions used in the present paper turn out to be more precise for estimating $\rho_{k}$ along the diagonal. The results of [3, 4] apply to the number of real roots of a Kostlan polynomial of degree $d$, see [24]. In this case, the variance asymptotics and the Central Limit Theorem were proved by Dalmao [17].

To conclude this section, let us mention that the setting of the present paper is related with that of $[3,4,21,29]$. Indeed, the Bargmann-Fock process introduced previously is the universal local scaling limit, as $d \rightarrow+\infty$, of a random section of degree $d$ in the complex Fubini-Study ensemble. See [4] for more details.

\subsection{Organization of the paper}

The content of this paper is organized as follows. In Section 2, we introduce our framework and the random measures $\nu_{R}$ we are interested in. We also introduce some useful notations that will appear throughout the paper. In Section 3, we recall the KacRice formulas. As first applications, we prove that the Kac-Rice density $\rho_{k}$ is the $k$-point function of the random point process $Z$ and Proposition 1.8. Section 4 is dedicated to the proof of the covariance estimates of Proposition 1.11. In Section 5, we introduce the divided differences associated with a function and study the distribution of the 
divided differences associated with a stationary Gaussian process. In Section 6, we use the divided differences to derive alternative expressions of the Kac-Rice densities. In particular, we prove Theorem 1.13 in Section 6.3 and Theorem 1.14 in Section 6.7. Section 7 is concerned with the proof of Theorem 1.6 and Section 8 is concerned with the proofs of the limit Theorems 1.16 and 1.21. This paper also contains three appendices. In Appendix A, we build examples of Gaussian processes satisfying the hypotheses of our main theorems. Appendix B contains the proofs of some auxiliary results related to the proof of Proposition 1.11. Finally, Appendix C is dedicated to the proof of a lemma pertaining to the regularity of the Kac-Rice densities.

\section{Framework}

In this section, we introduce the random measures we are interested in. First, in Section 2.1, we introduce some notations related to partitions of finite sets and diagonals in Cartesian products. In Section 2.2, we introduce properly the random processes we are interested in and their correlation functions. Finally, in Section 2.3, we prove that the vanishing locus of the processes introduced in Section 2.2 is almost surely well-behaved (see Lemma 2.12), and we introduce several counting measures associated with this random set.

\subsection{Partitions, products and diagonal inclusions}

Let us first introduce some notations that will be useful throughout the paper. Recall that we already defined the set $\mathcal{P}_{A}$ (resp. $\mathcal{P}_{k}$ ) of partitions of a finite set $A$ (resp. of $\{1, \ldots, k\}$ ) and the set $\mathcal{P P}_{A}$ (resp. $\mathcal{P P}_{k}$ ) of its partitions into pairs (see Definition 1.4).

Notations 2.1. Let $A$ be a finite set and let $Z$ be any set.

- We denote by $\operatorname{Card}(A)$ or by $|A|$ the cardinality of $A$.

- We denote by $Z^{A}$ the Cartesian product of $|A|$ copies of $Z$, indexed by the elements of $A$.

- A generic element of $Z^{A}$ is denoted by $\underline{x}_{A}=\left(x_{a}\right)_{a \in A}$, or more simply by $x$. If $B \subset A$ we denote by $\underline{x}_{B}=\left(x_{a}\right)_{a \in B}$.

- Let $\left(\phi_{a}\right)_{a \in A}$ be functions on $Z$, we denote by $\phi_{A}=\bigotimes_{a \in A} \phi_{a}$ the function on $Z^{A}$ defined by: $\phi_{A}\left(\underline{x}_{A}\right)=\prod_{a \in A} \phi_{a}\left(x_{a}\right)$, for all $\underline{x}_{A}=\left(x_{a}\right)_{a \in A} \in Z^{A}$. If $A$ is of the form $\{1, \ldots, k\}$ with $k \in \mathbb{N}^{*}$, we use the simpler notation $\phi=\phi_{A}$.

Definition 2.2 (Diagonals). Let $A$ be a non-empty finite set, we denote by $\Delta_{A}$ the large diagonal of $\mathbb{R}^{A}$ :

$$
\Delta_{A}=\left\{\left(x_{a}\right)_{a \in A} \in \mathbb{R}^{A} \mid \exists a, b \in A \text { such that } a \neq b \text { and } x_{a}=x_{b}\right\} .
$$

Moreover, for all $\mathcal{I} \in \mathcal{P}_{A}$, we denote by

$$
\Delta_{A, \mathcal{I}}=\left\{\left(x_{a}\right)_{a \in A} \in \mathbb{R}^{A} \mid \forall a, b \in A,\left(x_{a}=x_{b} \Longleftrightarrow \exists I \in \mathcal{I} \text { such that } a \in I \text { and } b \in I\right)\right\} .
$$

If $A=\{1, \ldots, k\}$, we use the simpler notations $\Delta_{k}=\Delta_{A}$ and $\Delta_{k, \mathcal{I}}=\Delta_{A, \mathcal{I}}$.

Definition 2.3 (Diagonal inclusions). Let $A$ be a non-empty finite set and let $\mathcal{I} \in \mathcal{P}_{A}$. The diagonal inclusion $\iota_{\mathcal{I}}$ is the function from $\mathbb{R}^{\mathcal{I}}$ to $\mathbb{R}^{A}$ defined by: for all $\underline{x}_{\mathcal{I}}=\left(x_{I}\right)_{I \in \mathcal{I}} \in \mathbb{R}^{\mathcal{I}}$, $\iota_{\mathcal{I}}\left(\underline{x}_{\mathcal{I}}\right)=\underline{y}_{A}=\left(y_{a}\right)_{a \in A}$, where for all $I \in \mathcal{I}$, for all $a \in I$, we set $y_{a}=x_{I}$.

Remark 2.4. With the previous definitions, we have $\mathbb{R}^{A}=\bigsqcup_{\mathcal{I} \in \mathcal{P}_{A}} \Delta_{A, \mathcal{I}}$ and similarly $\Delta_{A}=\bigsqcup_{\mathcal{I} \in \mathcal{P}_{A} \backslash\left\{\mathcal{I}_{\min }(A)\right\}} \Delta_{A, \mathcal{I}}$, where we denoted $\mathcal{I}_{\min }(A)=\{\{a\} \mid a \in A\}$ (this notation comes from the fact that $\mathcal{I}_{\min }(A)$ is the minimum of $\mathcal{P}_{A}$ for some partial order, see Definition 6.3). Moreover, for all $\mathcal{I} \in \mathcal{P}_{A}$, the map $\iota_{\mathcal{I}}$ is a smooth diffeomorphism from 
$\mathbb{R}^{\mathcal{I}} \backslash \Delta_{\mathcal{I}}$ onto $\Delta_{A, \mathcal{I}} \subset \mathbb{R}^{A}$. Note that $\Delta_{A, \mathcal{I}_{\min }(A)}$ is the configuration space $\mathbb{R}^{A} \backslash \Delta_{A}$ of $|A|$ distinct points in $\mathbb{R}$. In the following, we avoid using the notation $\Delta_{A, \mathcal{I}_{\min }(A)}$ and use $\mathbb{R}^{A} \backslash \Delta_{A}$ instead.

Remark 2.5. Let $y \in \mathbb{R}^{k}$, the partition $\mathcal{I}$ defined in Theorem 1.13 is the unique $\mathcal{I} \in \mathcal{P}_{k}$ such that $y \in \Delta_{k, \mathcal{I}}$. With the notations of Theorem 1.13, there exists $\left(y_{I}\right)_{I \in \mathcal{I}} \in \mathbb{R}^{\mathcal{I}} \backslash \Delta_{\mathcal{I}}$ such that $y=\iota_{\mathcal{I}}\left(\left(y_{I}\right)_{I \in \mathcal{I}}\right)$.

Let $Z \subset \mathbb{R}$ be a closed discrete subset. In particular, for any $K \subset \mathbb{R}$ compact, $Z \cap K$ is finite. As in the introduction, we denote by $\nu=\sum_{x \in Z} \delta_{x}$ the counting measure of $Z$. More generally, for any non-empty finite set $A$, we can define the counting measure of $Z^{A} \subset \mathbb{R}^{A}$.

Definition 2.6 (Counting measures). Let $Z \subset \mathbb{R}$ be closed and discrete and let $A$ be a non-empty finite set. We denote by:

$$
\nu^{A}=\sum_{x \in Z^{A}} \delta_{x} \quad \text { and } \quad \nu^{[A]}=\sum_{x \in Z^{A} \backslash \Delta_{A}} \delta_{x},
$$

where $\delta_{x}$ is the unit Dirac mass at $x \in \mathbb{R}^{A}$ and $\Delta_{A}$ is defined by Definition 2.2. These counting measures act on a function $\phi: \mathbb{R}^{A} \rightarrow \mathbb{R}$ as follows:

- if $\phi \geqslant 0$ or $\sum_{x \in Z^{A}}|\phi(x)|<+\infty$ then $\left\langle\nu^{A}, \phi\right\rangle=\sum_{x \in Z^{A}} \phi(x)$,

- if $\phi \geqslant 0$ or $\sum_{x \in Z^{A} \backslash \Delta_{A}}|\phi(x)|<+\infty$ then $\left\langle\nu^{[A]}, \phi\right\rangle=\sum_{x \in Z^{A} \backslash \Delta_{A}} \phi(x)$,

Quantities of the form $\left\langle\nu^{A}, \phi\right\rangle$ (resp. $\left\langle\nu^{[A]}, \phi\right\rangle$ ) are called the linear statistics of $\nu^{A}$ (resp. $\nu^{[A]}$ ). As usual, if $A=\{1, \ldots, k\}$, we denote $\nu^{k}=\nu^{A}$ and $\nu^{[k]}=\nu^{[A]}$.

Note that $\nu^{A}$ (resp. $\nu^{[A]}$ ) defines a Radon measure on $\mathbb{R}^{A}$, that is a continuous linear form on $\left(\mathcal{C}_{c}^{0}\left(\mathbb{R}^{A}\right),\|\cdot\|_{\infty}\right)$, the space of compactly supported continuous functions on $\mathbb{R}^{A}$ equipped with the sup-norm. Note also that the measure $\nu^{A}$ and $\nu^{[A]}$ are completely characterized by the linear statistics $\left\{\left\langle\nu^{A}, \phi\right\rangle \mid \phi \in \mathcal{C}_{c}^{0}\left(\mathbb{R}^{A}\right)\right\}$ and $\left\{\left\langle\nu^{[A]}, \phi\right\rangle \mid \phi \in \mathcal{C}_{c}^{0}\left(\mathbb{R}^{A}\right)\right\}$ respectively.

Lemma 2.7. Let $Z \subset \mathbb{R}$ be closed and discrete and let $A$ be a non-empty finite set. Using the notations introduced above, we have $\nu^{A}=\sum_{\mathcal{I} \in \mathcal{P}_{A}}\left(\iota_{\mathcal{I}}\right)_{*} \nu^{[\mathcal{I}]}$.

Proof. Recall that $\mathbb{R}^{A}=\bigsqcup_{\mathcal{I} \in \mathcal{P}_{A}} \Delta_{A, \mathcal{I}}$. Then, we have:

$$
\nu^{A}=\sum_{x \in Z^{A}} \delta_{x}=\sum_{\mathcal{I} \in \mathcal{P}_{A}}\left(\sum_{x \in Z^{A} \cap \Delta_{A, \mathcal{I}}} \delta_{x}\right) .
$$

Let $\mathcal{I} \in \mathcal{P}_{A}$, recall that $\iota_{\mathcal{I}}$ defines a smooth diffeomorphism from $\mathbb{R}^{\mathcal{I}} \backslash \Delta_{\mathcal{I}}$ onto $\Delta_{A, \mathcal{I}}$. Moreover, $\iota_{\mathcal{I}}\left(Z^{\mathcal{I}} \backslash \Delta_{\mathcal{I}}\right)=Z^{A} \cap \Delta_{A, \mathcal{I}}$ (see Definition 2.2 and 2.3). Hence,

$$
\sum_{x \in Z^{A} \cap \Delta_{A, \mathcal{I}}} \delta_{x}=\sum_{y \in Z^{\mathcal{I}} \backslash \Delta_{\mathcal{I}}} \delta_{\iota_{\mathcal{I}}(y)}=\sum_{y \in Z^{\mathcal{I}} \backslash \Delta_{\mathcal{I}}}\left(\iota_{\mathcal{I}}\right)_{*} \delta_{y}=\left(\iota_{\mathcal{I}}\right)_{*} \nu^{[\mathcal{I}]} .
$$

\subsection{Stationary Gaussian processes and correlation functions}

In this section, we introduce the random processes we study and how they are normalized. Let $f: \mathbb{R} \rightarrow \mathbb{R}$ be a stationary centered Gaussian process. By stationary, we mean that, for all $t \in \mathbb{R}$, the process $x \mapsto f(x+t)$ is distributed as $f$. Let $K: \mathbb{R}^{2} \rightarrow \mathbb{R}$ be the correlation kernel of $f$, defined by $K:(x, y) \mapsto \mathbb{E}[f(x) f(y)]$. Since $f$ is centered, its distribution is characterized by $K$. Let $\kappa: x \mapsto K(0, x)$ denote the correlation function of $f$. The stationarity of $f$ is equivalent to the fact that $K(x, y)=\kappa(y-x)$ for all $(x, y) \in \mathbb{R}^{2}$. Note that, since $K$ is symmetric, then $\kappa$ is an even function. 
Definition $2.8\left(\mathcal{C}^{p}\right.$-process). Let $p \in \mathbb{N} \cup\{\infty\}$, we say that $f$ is a process of class $\mathcal{C}^{p}$ (or a $\mathcal{C}^{p}$-process) if its trajectories are almost surely of class $\mathcal{C}^{p}$.

Let us assume that $f$ is of class $\mathcal{C}^{p}$, for some $p \in \mathbb{N} \cup\{\infty\}$. For all $k \in\{0, \ldots, p\}$ we denote by $f^{(k)}$ the $k$-th derivative of $f$. We also use the usual notations $f^{\prime}=f^{(1)}$ and $f^{\prime \prime}=f^{(2)}$. Then, for all $m \in \mathbb{N}^{*}$, for all $x_{1}, \ldots, x_{m} \in \mathbb{R}$, for all $k_{1}, \ldots, k_{m} \in\{0, \ldots, p\}$, the random vector $\left(f^{\left(k_{j}\right)}\left(x_{j}\right)\right)_{1 \leqslant j \leqslant m}$ is a centered Gaussian vector in $\mathbb{R}^{m}$. Let us denote by $\partial_{1}$ (resp. $\partial_{2}$ ) the partial derivative with respect to the first (resp. second) variable for functions from $\mathbb{R}^{2}$ to $\mathbb{R}$. For all $k$ and $l$ in $\{0, \ldots, p\}$, the partial derivative $\partial_{1}^{k} \partial_{2}^{l} K$ is well-defined and continuous on $\mathbb{R}^{2}$. Moreover, $\kappa$ is of class $\mathcal{C}^{2 p}$ and, for all $k, l \in\{0, \ldots, p\}$, for all $x, y \in \mathbb{R}$, we have:

$$
\mathbb{E}\left[f^{(k)}(x) f^{(l)}(y)\right]=\partial_{1}^{k} \partial_{2}^{l} K(x, y)=(-1)^{k} \kappa^{(k+l)}(y-x)
$$

In particular, the variance matrix of $\left(f^{\left(k_{j}\right)}\left(x_{j}\right)\right)_{1 \leqslant j \leqslant m}$ is $\left(\partial_{1}^{k_{i}} \partial_{2}^{k_{j}} K\left(x_{i}, x_{j}\right)\right)_{1 \leqslant i, j \leqslant m}$. This material is standard. We refer the interested reader to [31, Appendix A.2 and A.3] for more details.

Let us now assume that $f$ is a $\mathcal{C}^{1}$-process. If $\kappa(0)=0$, then for all $x \in \mathbb{R}$, almost surely $f(x)=0$. Then, almost surely, $f$ is continuous and for all $x \in \mathbb{Q}, f(x)=0$. Hence $f$ is almost surely the zero function. Similarly, if $\kappa^{\prime \prime}(0)=0$ then $f^{\prime}$ is almost surely the zero function. Hence $f$ is almost surely constant, equal to $f(0) \sim \mathcal{N}(0, \kappa(0))$. These degenerate situations are well-understood, and we will not consider them in the following. That is, we assume that $\operatorname{Var}(f(0))=\kappa(0)>0$ and $\operatorname{Var}\left(f^{\prime}(0)\right)=-\kappa^{\prime \prime}(0)>0$. Without loss of generality, up to replacing $f$ by:

$$
x \longmapsto \frac{1}{\sqrt{\kappa(0)}} f\left(\sqrt{-\frac{\kappa(0)}{\kappa^{\prime \prime}(0)}} x\right),
$$

we may assume that $\kappa(0)=1=-\kappa^{\prime \prime}(0)$.

Definition 2.9 (Normalization). We say that a stationary centered Gaussian process $f$ of class $\mathcal{C}^{1}$ is normalized if its correlation function $\kappa$ satisfies $\kappa(0)=1=-\kappa^{\prime \prime}(0)$.

In the rest of this paper, unless otherwise specified, the random process $f$ is always assumed to be a normalized stationary centered Gaussian process at least of class $\mathcal{C}^{1}$.

Recall that, in Theorems 1.6 and 1.14, we consider a normalized Gaussian $\mathcal{C}^{k}$-process $f$ whose correlation function $\kappa$ satisfies some form of decay at infinity, as well as its first derivatives. In the remainder of this section, we discuss these conditions. Let us first check that they make sense. Let $l \in\{0, \ldots, k\}$, for all $x \in \mathbb{R}$ we have:

$$
\kappa^{(2 l)}(x)^{2}=\mathbb{E}\left[f^{(l)}(0) f^{(l)}(x)\right]^{2} \leqslant \mathbb{E}\left[f^{(l)}(0)^{2}\right] \mathbb{E}\left[f^{(l)}(x)^{2}\right] \leqslant \kappa^{(2 l)}(0)^{2},
$$

and, if $l<k$,

$$
\kappa^{(2 l+1)}(x)^{2}=\mathbb{E}\left[f^{(l+1)}(0) f^{(l)}(x)\right]^{2} \leqslant \mathbb{E}\left[f^{(l+1)}(0)^{2}\right] \mathbb{E}\left[f^{(l)}(x)^{2}\right] \leqslant \kappa^{(2 l+2)}(0) \kappa^{(2 l)}(0) .
$$

Hence, $\kappa$ and all its derivatives of order at most $2 k$ are bounded on $\mathbb{R}$. Recalling Notation 1.3, this means that $\|\kappa\|_{l, \eta}$ is well-defined for any $l \in\{0, \ldots, 2 k\}$ and $\eta \geqslant 0$. Moreover, the previous inequalities show that $\|\kappa\|_{2 k}=\max \left\{\kappa^{(2 l)}(0) \mid 0 \leqslant l \leqslant k\right\}$. Note that asking for $\|\kappa\|_{k, \eta}$ to decay at some rate as $\eta \rightarrow+\infty$, is just a way to require that $\kappa$ and all its derivatives of order at most $k$ decay at said rate at infinity. For example, taking into account the parity of $\kappa$, the hypothesis that $\|\kappa\|_{k, \eta} \underset{\eta \rightarrow+\infty}{\longrightarrow} 0$ in Theorem 1.14 is equivalent to asking that $\kappa^{(k)}(x) \underset{x \rightarrow+\infty}{\longrightarrow} 0$ for all $l \in\{0, \ldots, k\}$. 
The fact that $\kappa$ tends to 0 infinity ensures the non-degeneracy of the finite-dimensional marginal distributions of the process $f$. Let us make this statement precise.

Lemma 2.10 (Non-degeneracy of the marginals). Let $p \in \mathbb{N}$ and let $f$ be a stationary centered Gaussian process of class $\mathcal{C}^{p}$ whose correlation function is denoted by $\kappa$. Let us assume that $\kappa(x) \underset{x \rightarrow+\infty}{\longrightarrow} 0$. Let $m \in \mathbb{N}^{*}$, let $x_{1}, \ldots, x_{m} \in \mathbb{R}$ and let $k_{1}, \ldots, k_{m} \in\{0, \ldots, p\}$ be such that the couples $\left(\left(x_{j}, k_{j}\right)\right)_{1 \leqslant j \leqslant m}$ are pairwise distinct. Then, the random vector $\left(f^{\left(k_{j}\right)}\left(x_{j}\right)\right)_{1 \leqslant j \leqslant m}$ is a non-degenerate centered Gaussian vector in $\mathbb{R}^{m}$.

Proof. Let us just sketch the proof here. The details are given in Appendix A.2. The condition that $\kappa$ tends to 0 at infinity implies that the process $f$ is ergodic, which is equivalent to the fact that its spectral measure has no atom. In particular, the spectral measure of $f$ has an accumulation point. This condition is enough to ensure the non-degeneracy of $\left(f^{\left(k_{j}\right)}\left(x_{j}\right)\right)_{1 \leqslant j \leqslant m}$ as soon as the couples $\left(\left(x_{j}, k_{j}\right)\right)_{1 \leqslant j \leqslant m}$ are pairwise distinct.

We conclude this section by making a few remarks about the content of this section and its relation to the hypotheses of Theorem 1.13 .

Remark 2.11. Let $f$ be a normalized stationary centered Gaussian process and let $\kappa$ denote its correlation function.

- Since $\kappa$ is even, $\kappa^{\prime}(0)=0$. In particular, for all $x \in \mathbb{R}$, the random vector $\left(f(x), f^{\prime}(x)\right)$ is a standard Gaussian vector in $\mathbb{R}^{2}$. That is, for all $x \in \mathbb{R}, f(x)$ and $f^{\prime}(x)$ are independent $\mathcal{N}(0,1)$ variables.

- Let $x, y \in \mathbb{R}$ be such that $x \neq y$, the determinant of the variance matrix of $(f(x), f(y))$ equals $1-\kappa(y-x)^{2}$. Hence, this Gaussian vector is degenerate if and only if $|\kappa(y-x)|=1$. By Cauchy-Schwarz's inequality, we have $|\kappa(x)| \leqslant \kappa(0)=1$ for all $x \in \mathbb{R}$. Thus, for $k=2$, the first non-degeneracy condition in Theorem 1.13 is equivalent to the fact that $|\kappa(x)|<1$ for any $x \neq 0$.

- Let $k \in \mathbb{N}^{*}$, if $f$ is of class $\mathcal{C}^{k}$ then, by Lemma 2.10, the fact $\kappa(x) \underset{x \rightarrow+\infty}{\longrightarrow} 0$ is enough to ensure that $f$ satisfies the hypotheses of Theorem 1.13 at any point $y \in \mathbb{R}^{k}$. This condition is sufficient but not necessary, see Lemma A.2 below.

\subsection{Zeros of stationary Gaussian processes}

Let us now introduce more precisely the random sets we study. Let $f$ be a normalized centered stationary Gaussian process and let us denote by $Z=f^{-1}(0)$ its vanishing locus.

Lemma 2.12. Let $f: \mathbb{R} \rightarrow \mathbb{R}$ be a normalized centered stationary Gaussian process and let $Z=f^{-1}(0)$. Then, almost surely, $Z$ is a closed discrete subset of $\mathbb{R}$.

Proof. The process $f$ is almost surely of class $\mathcal{C}^{1}$. By Bulinskaya's Lemma (see [6, Proposition. 1.20]), since $f(x) \sim \mathcal{N}(0,1)$ for all $x \in \mathbb{R}$, we have that $f$ vanishes transversally almost surely. That is, almost surely, for all $x \in \mathbb{R}$ such that $f(x)=0$ we have $f^{\prime}(x) \neq 0$. Then, $Z$ is almost surely a closed 0 -dimensional submanifold of $\mathbb{R}$. Equivalently, $Z$ is almost surely a closed discrete subset of $\mathbb{R}$.

Definition 2.13. Let $R>0$.

- We set $Z_{R}=\frac{1}{R} Z=\{x \in \mathbb{R} \mid f(R x)=0\}$.

- Let $\nu_{R}=\sum_{x \in Z_{R}} \delta_{x}$ (resp. $\nu=\sum_{x \in Z} \delta_{x}$ ) denote the counting measure of $Z_{R}$ (resp. Z).

- As in Definition 2.6, for any non-empty finite set $A$, we denote by $\nu^{A}$ (resp. $\nu^{[A]}$ ) the counting measure of the random set $Z^{A}$ (resp. $\left.Z^{A} \backslash \Delta_{A}\right)$. 
In this paper, we study the asymptotic distribution of $\nu_{R}$ as $R \rightarrow+\infty$ through the asymptotics of its linear statistics $\left\langle\nu_{R}, \phi\right\rangle$, where $\phi: \mathbb{R} \rightarrow \mathbb{R}$ is a nice enough function.

Notations 2.14. As in Section 1, we will use the following notations.

- Let $\Phi: \mathbb{R}^{A} \rightarrow \mathbb{R}$, for any $R>0$ we set $\Phi_{R}: x \mapsto \phi\left(\frac{x}{R}\right)$. In particular, if $\phi: \mathbb{R} \rightarrow \mathbb{R}$, we have $\left\langle\nu_{R}, \phi\right\rangle=\left\langle\nu, \phi_{R}\right\rangle$.

- Let $A$ be a subset of some set $B$, we denote by $\mathbb{1}_{A}: B \rightarrow \mathbb{R}$ the indicator function of $A$. For example, if $A \subset \mathbb{R}$, we have $\operatorname{Card}(Z \cap A)=\left\langle\nu, \mathbb{1}_{A}\right\rangle$.

Remark 2.15. Let $k \in \mathbb{N}^{*}$, then $\nu^{[k]}$ is the counting measure of $Z^{k} \backslash \Delta_{k}$. Let $B$ be a Borel subset of $\mathbb{R}$, we denote by $\mathcal{N}_{B}=\operatorname{Card}(Z \cap B)=\left\langle\nu, \mathbb{1}_{B}\right\rangle$. The $k$-th factorial moment of $\mathcal{N}_{B}$ is defined as the expectation of $\mathcal{N}_{B}^{[k]}=\mathcal{N}_{B}\left(\mathcal{N}_{B}-1\right) \cdots\left(\mathcal{N}_{B}-k+1\right)$. As explained in [6, p. 58], we have $\mathcal{N}_{B}^{[k]}=\left\langle\nu^{[k]}, \bigotimes_{i=1}^{k} \mathbb{1}_{B}\right\rangle=\left\langle\nu^{[k]}, \mathbb{1}_{B^{k}}\right\rangle$, hence $\mathbb{E}\left[\left\langle\nu^{[k]}, \mathbb{1}_{B^{k}}\right\rangle\right]$ is the $k$-th factorial moment of $\operatorname{Card}(Z \cap B)$. We will see below that this quantities are well-defined in $[0,+\infty]$.

\section{Kac-Rice formulas and mean number of zeros}

In this section, we state the so-called Kac-Rice formulas, that are one of the tools in the proofs of Theorem 1.6 and Propositions 1.8 and 1.11. The Kac-Rice formulas are recalled in Section 3.1. In Section 3.2, we related the Kac-Rice density introduced in Definition 3.1 with the $k$-point function of the random set $Z=f^{-1}(0)$ defined in Section 2.3. Then, in Section 3.3, we prove Proposition 1.8.

\subsection{Kac-Rice formulas}

In this section, we recall the Kac-Rice formulas (see Proposition 3.6). A standard reference for this material is [6, Chapters 3 and 6], see also [2, Chapter 11]. Note however that formulas of this kind already appear in the work of Cramér and Leadbetter [13].

First, we need to introduce the Kac-Rice densities associated with a non-degenerate Gaussian process of class $\mathcal{C}^{1}$.

Definition 3.1 (Kac-Rice densities). Let $f$ be a centered Gaussian $\mathcal{C}^{1}$-process. Let $k \in \mathbb{N}^{*}$ and let $x=\left(x_{i}\right)_{1 \leqslant i \leqslant k} \in \mathbb{R}^{k}$. We denote by

$$
D_{k}(x)=\operatorname{det}\left(\operatorname{Var}\left(f\left(x_{1}\right), \ldots, f\left(x_{k}\right)\right)\right) .
$$

If $\left(f\left(x_{1}\right), \ldots, f\left(x_{k}\right)\right)$ is non-degenerate, i.e. if $D_{k}(x) \neq 0$, we denote by

$$
N_{k}(x)=\mathbb{E}\left[\prod_{i=1}^{k}\left|f^{\prime}\left(x_{i}\right)\right| \mid \forall i \in\{1, \ldots, k\}, f\left(x_{i}\right)=0\right],
$$

the conditional expectation of $\left|f^{\prime}\left(x_{1}\right)\right| \cdots\left|f^{\prime}\left(x_{k}\right)\right|$ given that $f\left(x_{1}\right)=\cdots=f\left(x_{k}\right)=0$, and by

$$
\rho_{k}(x)=\frac{N_{k}(x)}{(2 \pi)^{\frac{k}{2}} D_{k}(x)^{\frac{1}{2}}} .
$$

We refer to $\rho_{k}$ as the Kac-Rice density of order $k$ associated with $f$.

Remark 3.2. By Lemma 2.10, if $\kappa(x) \underset{x \rightarrow+\infty}{\longrightarrow} 0$ then, for all $k \in \mathbb{N}^{*}$ the Kac-Rice density $\rho_{k}$ is well-defined on $\mathbb{R}^{k} \backslash \Delta_{k}$. Note however that $D_{k}$ always vanishes along $\Delta_{k}$.

Example 3.3. Let $f$ be a normalized Gaussian process (see Definition 2.9)

- For all $x \in \mathbb{R}, f(x)$ and $f^{\prime}(x)$ are independent $\mathcal{N}(0,1)$ variables (see Remark 2.11). Hence, $D_{1}(x)=\operatorname{Var}(f(x))=1$ and $N_{1}(x)=\mathbb{E}\left[\left|f^{\prime}(x)\right|\right]=\sqrt{\frac{2}{\pi}}$. Thus, $\rho_{1}$ is constant equal to $\frac{1}{\pi}$. 
- Let $\kappa$ denote the correlation function of $f$. We have $D_{2}(x, y)=1-\kappa(y-x)^{2}$ for all $(x, y) \in \mathbb{R}^{2}$. Hence $\rho_{2}$ is well-defined on $\mathbb{R}^{2} \backslash \Delta_{2}$ if and only if $|\kappa(x)|<1$ for all $x \in \mathbb{R} \backslash\{0\}$.

Notation 3.4 (Symmetric group). Let $A$ be a non-empty finite set, we denote by $\mathfrak{S}_{A}$ the group of permutations of $A$. For all $\sigma \in \mathfrak{S}_{A}$ and $x=\left(x_{a}\right)_{a \in A} \in \mathbb{R}^{A}$, we denote by $\sigma \cdot x=\left(x_{\sigma(a)}\right)_{a \in A}$. If $A=\{1, \ldots, k\}$, we denote $\mathfrak{S}_{k}=\mathfrak{S}_{A}$ for simplicity.

Lemma 3.5 (Symmetry). Let $k \in \mathbb{N}^{*}$, we have $D_{k}(\sigma \cdot x)=D_{k}(x)$ for all $x \in \mathbb{R}^{k}$. Moreover, if $D_{k}(x) \neq 0$, then $N_{k}(\sigma \cdot x)=N_{k}(x)$ and $\rho_{k}(\sigma \cdot x)=\rho_{k}(x)$.

Proof. Let $\sigma \in \mathfrak{S}_{k}$ and let $\Sigma$ denote the linear map $x \mapsto \sigma \cdot x$. For all $x \in \mathbb{R}^{k}$, we have:

$D_{k}(\sigma \cdot x)=\operatorname{det} \operatorname{Var}\left(f\left(x_{\sigma(1)}\right), \ldots, f\left(x_{\sigma(k)}\right)\right)=\operatorname{det} \operatorname{Var}\left(\Sigma\left(f\left(x_{1}\right), \ldots, f\left(x_{k}\right)\right)\right)=\operatorname{det}(\Sigma)^{2} D_{k}(x)$.

Since the matrix of $\Sigma$ in the canonical basis of $\mathbb{R}^{k}$ is a permutation matrix, $\operatorname{det}(\Sigma)^{2}=1$. This proves that $D_{k}$ is symmetric on $\mathbb{R}^{k}$.

If $D_{k}(x) \neq 0$, the first point shows that $D_{k}(\sigma \cdot x) \neq 0$, so that $N_{k}(x), N_{k}(\sigma \cdot x)$, $\rho_{k}(x)$ and $\rho_{k}(\sigma \cdot x)$ are well-defined. To conclude the proof it is enough to check that $N_{k}(\sigma \cdot x)=N_{k}(x)$. This follows from the definition of $N_{k}$, see Equation (3.2).

We can now state the Kac-Rice formula itself.

Proposition 3.6 (Kac-Rice formula). Let $f$ be a centered Gaussian process of class $\mathcal{C}^{1}$ and let $Z$ denote its zero set. Let $k \in \mathbb{N}^{*}$ and let $\nu^{[k]}$ be the counting measure of $Z^{k} \backslash \Delta_{k}$. Let $U$ be an open subset of $\mathbb{R}^{k}$ such that, for all $x \in U \backslash \Delta_{k}, D_{k}(x) \neq 0$ (cf. Definition 3.1). Let $\Phi: \mathbb{R}^{k} \rightarrow \mathbb{R}$ be a Borel function supported in $U$ satisfying one of the following conditions:

- the function $\Phi$ is non-negative;

- the function $\Phi \rho_{k}$ is Lebesgue-integrable on $\mathbb{R}^{k}$;

- the random variable $\left\langle\nu^{[k]}, \Phi\right\rangle$ is integrable.

Then we have:

$$
\mathbb{E}\left[\left\langle\nu^{[k]}, \Phi\right\rangle\right]=\int_{x \in \mathbb{R}^{k}} \Phi(x) \rho_{k}(x) \mathrm{d} x,
$$

where $\mathrm{d} x$ denote the Lebesgue measure of $\mathbb{R}^{k}$.

Proof. We refer to [6, Theorem 3.2] for a proof of this result (see also [6, Theorem 6.2 and 6.3]). Our statement of the Kac-Rice formula differs from those that can be found in [6]. Let us comment upon the differences.

In [6], the authors are concerned with the so-called factorial moments of the number of zeros of $f$ in some Borel set $B \subset \mathbb{R}$. As we already explained in Remark 2.15, the $k$-th factorial moment of $\operatorname{Card}(Z \cap B)$ is $\mathbb{E}\left[\left\langle\nu^{[k]}, \mathbb{1}_{B^{k}}\right\rangle\right]$. Hence, Azaïs and Wschebor state and prove Proposition 3.6 in the case where $\Phi$ is the indicator function $\mathbb{1}_{B^{k}}$, where $B$ is an interval in [6, Theorem 3.2] and a Borel set in [6, Theorem 6.2 and 6.3]. Their proofs can be adapted to deal with a Borel function $\Phi$. Alternatively, once the result is proved for the indicator function of a Borel set, it also holds for simple functions. Then, we conclude by approximating the positive and negative part of $\Phi$ by simple functions and applying Beppo Levi's Monotone Convergence Theorem.

Remark 3.7. The only place where we use the Kac-Rice formula with $U \neq \mathbb{R}^{k}$ is the proof of Lemma 3.11, where we prove that $\rho_{k}$ coincides with the $k$-point function of $Z$. 
Remark 3.8. We prove below that, if $f$ is of class $\mathcal{C}^{k}$ and its correlation function $\kappa$ is such that $\|\kappa\|_{k, \eta}$ tends to 0 as $\eta \rightarrow+\infty$, then the function $\rho_{k}$ is bounded (see Theorem 1.14). In this case, the second condition in Proposition 3.6 can be replaced by the Lebesgueintegrability of $\Phi$ on $\mathbb{R}^{k}$. In particular, this implies that for any integrable function $\Phi: \mathbb{R}^{k} \rightarrow \mathbb{R}$ the random variable $\left\langle\nu^{[k]}, \Phi\right\rangle$ is almost surely well-defined.

\subsection{Kac-Rice density and $k$-point functions}

In this section, we show that the Kac-Rice density $\rho_{k}$ introduced in Definition 3.1 is in fact the $k$-point function of the point process $Z=f^{-1}(0)$ introduced in Section 2.3. First, we need to prove the continuity of $\rho_{k}$.

Lemma 3.9 (Continuity). Let $f$ be a centered Gaussian process of class $\mathcal{C}^{1}$. For all $k \in \mathbb{N}^{*}$, the maps $D_{k}, N_{k}$ and $\rho_{k}$ appearing in Definition 3.1 are continuous on their domains of definition.

Proof. Let $k \in \mathbb{N}^{*}$, for all $x=\left(x_{i}\right)_{1 \leqslant i \leqslant k} \in \mathbb{R}^{k}$, let us denote by $X_{k}(x)=\left(f\left(x_{i}\right)\right)_{1 \leqslant i \leqslant k}$ and $Y_{k}(x)=\left(f^{\prime}\left(x_{i}\right)\right)_{1 \leqslant i \leqslant k}$. Then, $\left(X_{k}(x), Y_{k}(x)\right)_{x \in \mathbb{R}^{k}}$ is a continuous centered Gaussian field with values in $\mathbb{R}^{2 k}$. We write the variance matrix of $\left(X_{k}(x), Y_{k}(x)\right)$ by square blocks of size $k$ as:

$$
\left(\begin{array}{cc}
\Theta_{k}(x) & { }^{\mathrm{t}} \Xi_{k}(x) \\
\Xi_{k}(x) & \Omega_{k}(x)
\end{array}\right)
$$

where $\Theta_{k}, \Xi_{k}$ and $\Omega_{k}$ are continuous on $\mathbb{R}^{k}$. Then, $D_{k}=\operatorname{det}\left(\Theta_{k}\right)$ is continuous on $\mathbb{R}^{k}$.

If $x \in \mathbb{R}^{k}$ is such that $D_{k}(x) \neq 0$, then $Y_{k}(x)$ given that $X_{k}(x)=0$ is a well-defined centered Gaussian vector of variance matrix $\Lambda_{k}(x)=\Omega_{k}(x)-\Xi_{k}(x) \Theta_{k}(x)^{-1}{ }^{\mathrm{t}} \Xi_{k}(x)$ (see [6, Proposition 1.2]). Note that $\Lambda_{k}$ is continuous on $\left\{x \in \mathbb{R}^{k} \mid D_{k}(x) \neq 0\right\}$. Then,

$$
N_{k}(x)=\mathbb{E}\left[\prod_{i=1}^{k}\left|Z_{i}(x)\right|\right]
$$

where $\left(Z_{1}(x), \ldots, Z_{k}(x)\right) \sim \mathcal{N}\left(0, \Lambda_{k}(x)\right)$. That is, $N_{k}(x)=\Pi_{k}\left(\Lambda_{k}(x)\right)$, where $\Pi_{k}$ is the map defined in Definition C.1. Since $\Pi_{k}$ is continuous (see Corollary C.3), the function $N_{k}$ is continuous on $\left\{x \in \mathbb{R}^{k} \mid D_{k}(x) \neq 0\right\}$, and so is $\rho_{k}$.

Let us now consider a normalized centered stationary Gaussian process $f$ which is $\mathcal{C}^{1}$. By Lemma 2.12, its zero set $Z$ is a discrete closed subset of $\mathbb{R}$ almost surely. That is $Z$ is random point process in $\mathbb{R}$.

Definition 3.10 ( $k$-point function). Let $x=\left(x_{i}\right)_{1 \leqslant i \leqslant k} \in \mathbb{R}^{k} \backslash \Delta_{k}$, the value at $x$ of the $k$-point function of a random point process $Z$ is defined as:

$$
\lim _{\varepsilon \rightarrow 0} \frac{1}{(2 \varepsilon)^{k}} \mathbb{E}\left[\prod_{i=1}^{k} \operatorname{Card}\left(Z \cap\left[x_{i}-\varepsilon, x_{i}+\varepsilon\right]\right)\right],
$$

if this limit is well-defined.

We can now make precise our claim that $\rho_{k}$ is the $k$-point function of $Z$.

Lemma 3.11. Let $k \in \mathbb{N}^{*}$ and let $f$ be a normalized centered stationary Gaussian $\mathcal{C}^{1}$ process. Let us denote by $Z$ the vanishing locus of $f$. Then, for all $x=\left(x_{i}\right)_{1 \leqslant i \leqslant k} \in \mathbb{R}^{k}$ such that $D_{k}(x) \neq 0$, we have:

$$
\frac{1}{(2 \varepsilon)^{k}} \mathbb{E}\left[\prod_{i=1}^{k} \operatorname{Card}\left(Z \cap\left[x_{i}-\varepsilon, x_{i}+\varepsilon\right]\right)\right] \underset{\varepsilon \rightarrow 0}{\longrightarrow} \rho_{k}(x),
$$

where $\rho_{k}$ is the function appearing in Definition 3.1. 
Proof. Since $D_{k}(x) \neq 0$, by continuity of $D_{k}$ (see Lemma 3.9) there exists a neighborhood $U$ of $x$ such that $D_{k}$ does not vanish on $U$. Note that this implies $U \subset \mathbb{R}^{k} \backslash \Delta_{k}$.

Let $\varepsilon \neq 0$. Without loss of generality, we can assume $\varepsilon$ to be positive and small enough that $\prod_{i=1}^{k}\left[x_{i}-\varepsilon, x_{i}+\varepsilon\right] \subset U \subset \mathbb{R}^{k} \backslash \Delta_{k}$. In particular, the intervals $\left(\left[x_{i}-\varepsilon, x_{i}+\varepsilon\right]\right)_{1 \leqslant i \leqslant k}$ are pairwise disjoint. Let $C$ denote the cube $[-1,1]^{k}$. Using the notations introduced in Definition 2.13 and Notations 2.14, we have:

$$
\prod_{i=1}^{k} \operatorname{Card}\left(Z \cap\left[x_{i}-\varepsilon, x_{i}+\varepsilon\right]\right)=\operatorname{Card}\left(Z^{k} \cap(x+\varepsilon C)\right)=\left\langle\nu^{k}, \mathbb{1}_{x+\varepsilon C}\right\rangle=\left\langle\nu^{[k]}, \mathbb{1}_{x+\varepsilon C}\right\rangle,
$$

since $x+\varepsilon C$ does not intersect $\Delta_{k}$. The function $\rho_{k}$ is well-defined and continuous on $U$. Then, by the Kac-Rice formula of order $k$ (see Proposition 3.6), we have:

$$
\frac{1}{(2 \varepsilon)^{k}} \mathbb{E}\left[\prod_{i=1}^{k} \operatorname{Card}\left(Z \cap\left[x_{i}-\varepsilon, x_{i}+\varepsilon\right]\right)\right]=\frac{1}{(2 \varepsilon)^{k}} \int_{x+\varepsilon C} \rho_{k}(y) \mathrm{d} y \underset{\varepsilon \rightarrow 0}{\longrightarrow} \rho_{k}(x),
$$

since $x+\varepsilon C$ has volume $(2 \varepsilon)^{k}$ and $\rho_{k}$ is continuous at $x$.

\subsection{Proof of Proposition 1.8: expectation of the linear statistics}

A first application of the Kac-Rice formulas (cf. Proposition 3.6) is the computation of the expectation of the linear statistics $\left\langle\nu_{R}, \phi\right\rangle$ (see Section 2.3), where $R>0$ and $\phi: \mathbb{R} \rightarrow \mathbb{R}$ is either non-negative or integrable. In this section, we address this problem and prove Proposition 1.8.

Proof of Proposition 1.8. Let $R>0$ and let $\phi: \mathbb{R} \rightarrow \mathbb{R}$ be non-negative or integrable. By definition of $\nu_{R}$ and $\phi_{R}$ (see Section 2.3), we have $\mathbb{E}\left[\left\langle\nu_{R}, \phi\right\rangle\right]=\mathbb{E}\left[\left\langle\nu, \phi_{R}\right\rangle\right]$. We apply the Kac-Rice formula for $k=1$, bearing in mind that $\rho_{1}$ is constant equal to $\frac{1}{\pi}$ (see Example 3.3). We obtain:

$$
\mathbb{E}\left[\left\langle\nu_{R}, \phi\right\rangle\right]=\mathbb{E}\left[\left\langle\nu, \phi_{R}\right\rangle\right]=\mathbb{E}\left[\left\langle\nu^{[1]}, \phi_{R}\right\rangle\right]=\frac{1}{\pi} \int_{-\infty}^{+\infty} \phi_{R}(x) \mathrm{d} x=\frac{R}{\pi} \int_{-\infty}^{+\infty} \phi(x) \mathrm{d} x .
$$

For all $\phi \in \mathcal{C}_{c}^{0}(\mathbb{R})$ we have: $\left\langle\mathbb{E}\left[\nu_{R}\right], \phi\right\rangle=\mathbb{E}\left[\left\langle\nu_{R}, \phi\right\rangle\right]=\left\langle\frac{R}{\pi} \mathrm{d} x, \phi\right\rangle$. Hence, $\mathbb{E}\left[\nu_{R}\right]=\frac{R}{\pi} \mathrm{d} x$.

As explained in Remark 1.9, applying Proposition 1.8 for the positive function $|\phi|$ allows to prove that, if $\phi$ is integrable then, for all $R>0,\left\langle\nu_{R}, \phi\right\rangle$ is almost surely welldefined. We can do a bit better than that. For example, let $\mathcal{E}$ denote the following space of functions:

$$
\mathcal{E}=\left\{\phi: \mathbb{R} \rightarrow \mathbb{R} \text { Lebesgue-measurable }|\exists C>0, \exists \alpha>1, \forall x \in \mathbb{R},| \phi(x) \mid \leqslant \frac{C}{1+|x|^{\alpha}}\right\} .
$$

For all $C>0$ and $\alpha>1$, we denote by $\psi_{C, \alpha}: x \mapsto \frac{C}{1+|x|^{\alpha}}$, from $\mathbb{R}$ to $\mathbb{R}$. By Proposition 1.8, for all $C>0$ and $\alpha>1$ such that $C \in \mathbb{Q}$ and $\alpha \in \mathbb{Q}$, we have $\left\langle\nu, \psi_{C, \alpha}\right\rangle<+\infty$ almost surely. Hence, almost surely, for all $\phi \in \mathcal{E}$, we have $\langle\nu,|\phi|\rangle<+\infty$. A function $\phi$ belongs to $\mathcal{E}$ if and only if it is bounded and $\phi(x)=O\left(|x|^{-\alpha}\right)$ as $|x| \rightarrow+\infty$, for some $\alpha>1$. Thus, if $\phi \in \mathcal{E}$, then $\phi_{R} \in \mathcal{E}$ for all $R>0$. Finally, we obtain that, almost surely, for all $\phi \in \mathcal{E}$, for all $R>0$, we have $\left\langle\nu,\left|\phi_{R}\right|\right\rangle\left\langle+\infty\right.$, i.e. $\left\langle\nu_{R}, \phi\right\rangle=\left\langle\nu, \phi_{R}\right\rangle$ is well-defined. Of course, in this example, the family $\left\{\psi_{C, \alpha} \mid C>0, \alpha>1\right\}$ can be replaced by any countable family of non-negative integrable functions. The same idea shows that $\nu_{R}$ almost surely defines a tempered distribution.

Lemma 3.12. Using the same notations as in Proposition 1.8, almost surely, for all $R>0$ we have $\nu_{R} \in \mathcal{S}^{\prime}(\mathbb{R})$. 
Proof. We recalled the definitions of $\mathcal{S}(\mathbb{R})$ and $\mathcal{S}^{\prime}(\mathbb{R})$ in Definition 1.18. For all $\phi \in \mathcal{S}(\mathbb{R})$, we denote by $C(\phi)=\max _{x \in \mathbb{R}^{n}}\left|\left(1+x^{2}\right) \phi(x)\right|$. Note that $\phi \mapsto C(\phi)$ is one of the norms defining the topology of $\mathcal{S}(\mathbb{R})$. In particular, $C(\phi) \rightarrow 0$ as $\phi \rightarrow 0$ in $\mathcal{S}(\mathbb{R})$.

Let $\psi: x \mapsto \frac{1}{1+x^{2}}$. By Proposition 1.8, almost surely $\langle\nu, \psi\rangle\langle+\infty$. Let us consider a fixed realization of $\nu$ in the full probability event such that $\langle\nu, \psi\rangle\langle+\infty$. For all $R>0$ and all $\phi \in \mathcal{S}(\mathbb{R})$ we have:

$$
\left\langle\nu_{R},|\phi|\right\rangle=\left\langle\nu,\left|\phi_{R}\right|\right\rangle \leqslant C\left(\phi_{R}\right)\langle\nu, \psi\rangle \leqslant C(\phi) R^{2}\langle\nu, \psi\rangle .
$$

On the one hand, this shows that $\left\langle\nu_{R}, \phi\right\rangle$ is well-defined. On the other hand, for all $R>0$,

$$
\left|\left\langle\nu_{R}, \phi\right\rangle\right| \leqslant C(\phi) R^{2}\langle\nu, \psi\rangle \underset{\phi \rightarrow 0}{\longrightarrow} 0 .
$$

Thus the linear form $\phi \mapsto\left\langle\nu_{R}, \phi\right\rangle$ is continuous on $\mathcal{S}(\mathbb{R})$, i.e. $\nu_{R} \in \mathcal{S}^{\prime}(\mathbb{R})$.

\section{Proof of Proposition 1.11: asymptotics of the covariances}

This section is concerned with the proof of Proposition 1.11. In all this section, we consider a Gaussian process $f$ satisfying the hypotheses of Proposition 1.11, that is $f$ is a normalized stationary centered Gaussian $\mathcal{C}^{2}$-process. Moreover, the correlation function $\kappa$ of $f$ is such that $\kappa$ and $\kappa^{\prime \prime}$ are square-integrable functions that tend to 0 at infinity.

First, in Section 4.1, we prove that the asymptotics given in Equation (1.1) holds. Then, we prove the positivity of the constant $\sigma$ (see Equation (1.2)) in Section 4.2.

\subsection{Asymptotics of the covariances}

In this section, we prove that Equation (1.1) in Proposition 1.11 holds. The content of this section is close to what can already be found in the literature, for example in the work of Cuzick [15]. The main difference is that we added test-functions $\phi_{1}$ and $\phi_{2}$ in Equation (1.1), where other authors generally consider the case $\phi_{1}=\phi_{2}=\mathbb{1}_{[0,1]}$. However, some of the notations and auxiliary results of this section will also be used in the proof of Theorem 1.6 (see Section 7.5). Besides, the proof of (1.1) is a good toy-model for the proof of Theorem 1.6, which is another reason to write it in full here.

We first introduce a density function $F$ (see Definition 4.1) and state some of its properties in Lemmas 4.2 and 4.3. The proofs of these lemmas are postponed until Appendix B. Then we establish Equation (1.1).

Since $\kappa$ tends to 0 at infinity, by Lemma 2.10, the Kac-Rice density $\rho_{2}$ is well-defined on $\mathbb{R}^{2} \backslash \Delta_{2}$ (see Remark 3.2). Moreover, since $f$ is stationary, we have $\rho_{2}(x, x+z)=\rho_{2}(0, z)$ for all $z \neq 0$ (see Definition 3.1).

Definition 4.1. We denote by $F: z \mapsto \rho_{2}(0, z)-\frac{1}{\pi^{2}}$ from $\mathbb{R} \backslash\{0\}$ to $\mathbb{R}$.

Note that, for all $x \neq y$, we have $\rho_{2}(x, y)-\rho_{1}(x) \rho_{1}(y)=F(y-x)$. It is possible to compute a somewhat more explicit expression of $F$.

Lemma 4.2. For all $z>0$, we have:

$$
\begin{aligned}
& \qquad F(z)=F(-z)=\frac{1}{\pi^{2}}\left(\frac{1-\kappa(z)^{2}-\kappa^{\prime}(z)^{2}}{\left(1-\kappa(z)^{2}\right)^{\frac{3}{2}}}\left(\sqrt{1-a(z)^{2}}+a(z) \arcsin (a(z))\right)-1\right), \\
& \text { where } a(z)=\frac{\kappa(z) \kappa^{\prime}(z)^{2}-\kappa(z)^{2} \kappa^{\prime \prime}(z)+\kappa^{\prime \prime}(z)}{1-\kappa(z)^{2}-\kappa^{\prime}(z)^{2}} \in[-1,1] .
\end{aligned}
$$

Proof. See Appendix B.1. 
Lemma 4.3. Under the hypotheses of Proposition 1.11, we have:

$$
F(z) \underset{z \rightarrow 0}{\longrightarrow}-\frac{1}{\pi^{2}} \quad \text { and } \quad F(z) \underset{|z| \rightarrow+\infty}{\longrightarrow} 0 .
$$

Moreover, the function $F$ is Lebesgue-integrable on $\mathbb{R}$.

Proof. See Appendix B.2.

Assuming that Lemmas 4.2 and 4.3 hold, we can now prove the first part of Proposition 1.11. An important step is the following lemma, which will also appear in the proof of Theorem 1.6.

Lemma 4.4. Under the hypotheses of Proposition 1.11, for all $R>0$ we have:

$$
m_{2}\left(\nu_{R}\right)\left(\phi_{1}, \phi_{2}\right)=\int_{\mathbb{R}^{2}} \phi_{1}\left(\frac{x}{R}\right) \phi_{2}\left(\frac{y}{R}\right) F(y-x) \mathrm{d} x \mathrm{~d} y+\frac{R}{\pi} \int_{\mathbb{R}} \phi_{1}(x) \phi_{2}(x) \mathrm{d} x,
$$

where $F$ is the function introduced in Definition 4.1 .

Proof. Let $R>0$ and let $\phi_{1}$ and $\phi_{2}$ be two Lebesgue-integrable functions such that $\phi_{2}$ is essentially bounded and continuous almost everywhere. Note that $\phi_{1} \phi_{2}$ is integrable. By Remark 1.9, the random variables $\left\langle\nu_{R}, \phi_{1}\right\rangle,\left\langle\nu_{R}, \phi_{2}\right\rangle$ and $\left\langle\nu_{R}, \phi_{1} \phi_{2}\right\rangle$ are almost surely well-defined and integrable. Using the Notations 2.1, we have $\phi_{R}=\left(\phi_{1}\right)_{R} \otimes\left(\phi_{2}\right)_{R}$ and:

$$
\begin{aligned}
m_{2}\left(\nu_{R}\right)\left(\phi_{1}, \phi_{2}\right) & =\mathbb{E}\left[\left\langle\nu_{R}, \phi_{1}\right\rangle\left\langle\nu_{R}, \phi_{2}\right\rangle\right]-\mathbb{E}\left[\left\langle\nu_{R}, \phi_{1}\right\rangle\right] \mathbb{E}\left[\left\langle\nu_{R}, \phi_{2}\right\rangle\right] \\
& =\mathbb{E}\left[\left\langle\nu^{2}, \phi_{R}\right\rangle\right]-\mathbb{E}\left[\left\langle\nu,\left(\phi_{1}\right)_{R}\right\rangle\right] \mathbb{E}\left[\left\langle\nu,\left(\phi_{2}\right)_{R}\right\rangle\right] \\
& =\mathbb{E}\left[\left\langle\nu^{[2]}, \phi_{R}\right\rangle\right]+\mathbb{E}\left[\left\langle\nu,\left(\phi_{1} \phi_{2}\right)_{R}\right\rangle\right]-\mathbb{E}\left[\left\langle\nu,\left(\phi_{1}\right)_{R}\right\rangle\right] \mathbb{E}\left[\left\langle\nu,\left(\phi_{2}\right)_{R}\right\rangle\right] .
\end{aligned}
$$

The middle term in the previous expression equals $\mathbb{E}\left[\left\langle\nu_{R}, \phi_{1} \phi_{2}\right\rangle\right]=\frac{R}{\pi} \int_{\mathbb{R}} \phi_{1}(x) \phi_{2}(x) \mathrm{d} x$, by Proposition 1.8. We compute the other two terms by the Kac-Rice formulas of order 1 and 2. By Lemma 3.9, $\rho_{2}$ is continuous on $\mathbb{R}^{2} \backslash \Delta_{2}$. By Lemma 4.3 and Definition 4.1, the function $\rho_{2}$ is bounded on $\mathbb{R}^{2} \backslash \Delta_{2}$. Thus, $\phi_{R} \rho_{2}$ is Lebesgue-integrable on $\mathbb{R}^{2}$. Then, by Lemma 2.10, the hypotheses of Proposition 3.6 are satisfied. Recalling that $\rho_{1}$ is constant equal to $\frac{1}{\pi}$ (see Example 3.3), we obtain:

$$
\begin{aligned}
\mathbb{E}\left[\left\langle\nu^{[2]}, \phi_{R}\right\rangle\right]-\mathbb{E}\left[\left\langle\nu,\left(\phi_{1}\right)_{R}\right\rangle\right] & \mathbb{E}\left[\left\langle\nu,\left(\phi_{2}\right)_{R}\right\rangle\right] \\
& =\int_{\mathbb{R}^{2}} \phi_{1}\left(\frac{x}{R}\right) \phi_{2}\left(\frac{y}{R}\right)\left(\rho_{2}(x, y)-\rho_{1}(x) \rho_{1}(y)\right) \mathrm{d} x \mathrm{~d} y \\
& =\int_{\mathbb{R}^{2}} \phi_{1}\left(\frac{x}{R}\right) \phi_{2}\left(\frac{y}{R}\right) F(y-x) \mathrm{d} x \mathrm{~d} y .
\end{aligned}
$$

Proof of Equation (1.1). Under the hypotheses of Proposition 1.11, we apply Lemma 4.4, which yields:

$$
m_{2}\left(\nu_{R}\right)\left(\phi_{1}, \phi_{2}\right)=\int_{\mathbb{R}^{2}} \phi_{1}\left(\frac{x}{R}\right) \phi_{2}\left(\frac{y}{R}\right) F(y-x) \mathrm{d} x \mathrm{~d} y+\frac{R}{\pi} \int_{\mathbb{R}} \phi_{1}(x) \phi_{2}(x) \mathrm{d} x .
$$

By a change of variable, we obtain:

$$
\int_{\mathbb{R}^{2}} \phi_{1}\left(\frac{x}{R}\right) \phi_{2}\left(\frac{y}{R}\right) F(y-x) \mathrm{d} x \mathrm{~d} y=R \int_{\mathbb{R}^{2}} \phi_{1}(x) \phi_{2}\left(x+\frac{z}{R}\right) F(z) \mathrm{d} x \mathrm{~d} z .
$$

Let us define $g:(x, z) \mapsto \phi_{1}(x) \phi_{2}(x) F(z)$ and $g_{R}:(x, z) \mapsto \phi_{1}(x) \phi_{2}\left(x+\frac{z}{R}\right) F(z)$ for all $R>0$. Since $\phi_{2}$ is continuous almost everywhere, $g_{R}$ simply converges toward $g$ almost everywhere on $\mathbb{R}^{2}$. Besides, for all $(x, z) \in \mathbb{R}^{2}$ we have:

$$
\left|g_{R}(x, z)\right| \leqslant\left\|\phi_{2}\right\|_{\infty}\left|\phi_{1}(x)\right||F(z)|,
$$


where $\left\|\phi_{2}\right\|_{\infty}$ stands for the essential supremum of $\phi_{2}$. Since $\phi_{1}$ and $F$ are integrable on $\mathbb{R}$ (see Lemma 4.3), the right-hand side is integrable on $\mathbb{R}^{2}$. By Lebesgue's Dominated Convergence Theorem, we get:

$$
\int_{\mathbb{R}^{2}} \phi_{1}(x) \phi_{2}\left(x+\frac{z}{R}\right) F(z) \mathrm{d} x \mathrm{~d} z \underset{R \rightarrow+\infty}{\longrightarrow}\left(\int_{x \in \mathbb{R}} \phi_{1}(x) \phi_{2}(x) \mathrm{d} x\right)\left(\int_{z \in \mathbb{R}} F(z) \mathrm{d} z\right) .
$$

Putting together everything we have done so far, as $R \rightarrow+\infty$, we have:

$$
m_{2}\left(\nu_{R}\right)\left(\phi_{1}, \phi_{2}\right)=R\left(\int_{-\infty}^{+\infty} \phi_{1}(x) \phi_{2}(x) \mathrm{d} x\right)\left(\frac{1}{\pi}+\int_{-\infty}^{+\infty} F(z) \mathrm{d} z\right)+o(R) .
$$

Finally, by Lemma 4.2 and Equation (1.2), we have: $\frac{1}{\pi}+\int_{-\infty}^{+\infty} F(z) \mathrm{d} z=\sigma^{2}$, hence the result.

\subsection{Positivity of the leading constant}

The goal of this section is to conclude the proof of Proposition 1.11, by proving that $\sigma^{2}>0$, see Corollary 4.8 below. Recall that $\sigma^{2}$ is given by Equation (1.2) and that $\sigma$ is its non-negative square root. It is not clear from its expression that $\sigma^{2}$ is positive. Indeed, Equation (1.2) can be rewritten (cf. Section 4.1) as:

$$
\sigma^{2}=\frac{1}{\pi}+2 \int_{0}^{+\infty} F(z) \mathrm{d} z
$$

where $F$ is defined by Definition 4.1. The function $F$ is not non-negative since it tends to $-\frac{1}{\pi^{2}}$ as $z \rightarrow 0$ (see Lemma 4.3). In fact, on several examples $2 \int_{0}^{+\infty} F(z) \mathrm{d} z<0$ and we would need to compare this integral with $-\frac{1}{\pi}$ in order to deduce the positivity of $\sigma^{2}$ from the previous expression.

Our proof does not use Equation (1.2), but relies on the Wiener-Itô expansion of $\left\langle\nu_{R}, \mathbb{1}_{[0,1]}\right\rangle$ derived by Kratz-Leòn in [26]. It is not necessary to know about these Wiener-Itô expansions to understand what follows, and we refer the interested reader to [26].

Proposition 4.5. Let $f$ be a normalized centered stationary Gaussian $\mathcal{C}^{2}$-process and let $Z$ denote its zero set. Then, for any $R>0$, there exists a square-integrable centered random variable $X_{R}$ such that:

$$
\operatorname{Card}(Z \cap[0, R])=\frac{R}{\pi}+\frac{1}{2 \pi} \int_{0}^{R} f^{\prime}(x)^{2}-f(x)^{2} \mathrm{~d} x+X_{R} .
$$

Moreover, $\int_{0}^{R} f^{\prime}(x)^{2}-f(x)^{2} \mathrm{~d} x$ is a square-integrable centered random variable and we have:

$$
\mathbb{E}\left[\left(\int_{0}^{R} f^{\prime}(x)^{2}-f(x)^{2} \mathrm{~d} x\right) X_{R}\right]=0 .
$$

Remark 4.6. One can check that, since $f$ is normalized (see Definition 2.9), we have:

$$
\mathbb{E}\left[\int_{0}^{R} f(x)^{2} \mathrm{~d} x\right]=\int_{0}^{R} \mathbb{E}\left[f(x)^{2}\right] \mathrm{d} x=\int_{0}^{R} \mathrm{~d} x=R
$$

and, by Cauchy-Schwarz's Inequality:

$$
\mathbb{E}\left[\left(\int_{0}^{R} f(x)^{2} \mathrm{~d} x\right)^{2}\right]=\int_{0}^{R} \int_{0}^{R} \mathbb{E}\left[f(x)^{2} f(y)^{2}\right] \mathrm{d} x \mathrm{~d} y \leqslant\left(\int_{0}^{R} \mathbb{E}\left[f(x)^{4}\right]^{\frac{1}{2}} \mathrm{~d} x\right)^{2}=3 R^{2} .
$$


Thus $\int_{0}^{R} f(x)^{2} \mathrm{~d} x$ is square-integrable of mean $R$. Similarly, $\int_{0}^{R} f^{\prime}(x)^{2} \mathrm{~d} x$ is squareintegrable of mean $R$. Hence the difference of these terms is indeed square-integrable and centered.

Proof of Proposition 4.5. This result is a simplified version of [26, Proposition 1]. Note that this result holds for a normalized process $f$ whose correlation function $\kappa$ satisfies $\kappa^{(4)}(0)<+\infty$ (see [26, Condition (1), p. 238]). Here, our process $f$ is of class $\mathcal{C}^{2}$, hence this condition is satisfied.

Let us denote by $\mathcal{N}_{R}=\operatorname{Card}(Z \cap[0, R])$. Kratz and Leòn prove that an expansion of the form:

$$
\mathcal{N}_{R}=\mathbb{E}\left[\mathcal{N}_{R}\right]+\sum_{q \geqslant 1} \mathcal{N}_{R}[q]
$$

holds in the space of $L^{2}$-random variables, where the $\left(\mathcal{N}_{R}[q]\right)_{q \geqslant 1}$ are uncorrelated centered random variables. Using Proposition 1.8 and setting $X_{R}=\sum_{q \geqslant 2} \mathcal{N}_{R}[q]$, we have:

$$
\mathcal{N}_{R}=\frac{R}{\pi}+\mathcal{N}_{R}[1]+X_{R}
$$

where $\mathcal{N}_{R}[1]$ and $X_{R}$ are centered $L^{2}$-random variables such that $\mathbb{E}\left[\mathcal{N}_{R}[1] X_{R}\right]=0$. Then, [26, Proposition 1] gives an expression of $\mathcal{N}_{R}[q]$, for all $q \geqslant 0$. In particular, we have:

$$
\mathcal{N}_{R}[1]=a_{0} b_{2}(0) \int_{0}^{R} H_{2}(f(x)) H_{0}\left(f^{\prime}(x)\right) \mathrm{d} x+a_{2} b_{0}(0) \int_{0}^{R} H_{0}(f(x)) H_{2}\left(f^{\prime}(x)\right) \mathrm{d} x .
$$

Here $H_{0}(X)=1$ and $H_{2}(X)=X^{2}-1$ are the Hermite polynomials of degree 0 and 2 respectively, $a_{0}=\sqrt{\frac{2}{\pi}}$ and $a_{2}=\frac{1}{\sqrt{2 \pi}}$ by [26, Lemma 2], $b_{0}(0)=\frac{1}{\sqrt{2 \pi}}$ and $b_{2}(0)=\frac{1}{\sqrt{8 \pi}}$ by [26, Proposition 1]. Finally, a direct computation yields:

$$
\mathcal{N}_{R}[1]=\frac{1}{2 \pi} \int_{0}^{R} f^{\prime}(x)^{2}-f(x)^{2} \mathrm{~d} x
$$

Lemma 4.7. Let $f$ be a normalized centered stationary Gaussian $\mathcal{C}^{2}$-process and let $\kappa$ denote its correlation function. We assume that $\kappa$ and $\kappa^{\prime \prime}$ are square-integrable and that $\kappa(x) \kappa^{\prime}(x) \rightarrow 0$ as $x \rightarrow+\infty$. Then,

$$
\frac{1}{R} \operatorname{Var}\left(\int_{0}^{R} f^{\prime}(x)^{2}-f(x)^{2} \mathrm{~d} x\right) \underset{R \rightarrow+\infty}{\longrightarrow} 4 \int_{0}^{+\infty}\left(\kappa(x)+\kappa^{\prime \prime}(x)\right)^{2} \mathrm{~d} x .
$$

Proof. As explained in Remark 4.6, $\int_{0}^{R} f^{\prime}(x)^{2}-f(x)^{2} \mathrm{~d} x$ is square-integrable and centered. For all $R>0$, we have:

$$
\begin{aligned}
& \operatorname{Var}\left(\int_{0}^{R} f^{\prime}(x)^{2}-f(x)^{2} \mathrm{~d} x\right)=\mathbb{E}\left[\left(\int_{0}^{R} f^{\prime}(x)^{2}-f(x)^{2} \mathrm{~d} x\right)^{2}\right] \\
& \quad=\int_{0}^{R} \int_{0}^{R} \mathbb{E}\left[f^{\prime}(x)^{2} f^{\prime}(y)^{2}\right]-\mathbb{E}\left[f^{\prime}(x)^{2} f(y)^{2}\right]-\mathbb{E}\left[f(x)^{2} f^{\prime}(y)^{2}\right]+\mathbb{E}\left[f(x)^{2} f(y)^{2}\right] \mathrm{d} x \mathrm{~d} y .
\end{aligned}
$$

By Wick's Formula (see [2, Lemma 11.6.1]), if $(X, Y)$ is a centered Gaussian vector in $\mathbb{R}^{2}$, then we have $\mathbb{E}\left[X^{2} Y^{2}\right]=\mathbb{E}\left[X^{2}\right] \mathbb{E}\left[Y^{2}\right]+2 \mathbb{E}[X Y]^{2}$. For example, using the stationarity and normalization of $f$, we have:

$$
\mathbb{E}\left[f(x)^{2} f(y)^{2}\right]=\mathbb{E}\left[f(x)^{2}\right] \mathbb{E}\left[f(y)^{2}\right]+2 \mathbb{E}[f(x) f(y)]^{2}=1+2 \kappa(y-x)^{2} .
$$


Applying Wick's Formula to $(f(x), f(y)),\left(f(x), f^{\prime}(y)\right),\left(f^{\prime}(x), f(y)\right)$ and $\left(f^{\prime}(x), f^{\prime}(y)\right)$ yields:

$$
\begin{aligned}
\operatorname{Var}\left(\int_{0}^{R} f^{\prime}(x)^{2}-f(x)^{2} \mathrm{~d} x\right) & =2 \int_{0}^{R} \int_{0}^{R} \kappa^{\prime \prime}(y-x)^{2}-2 \kappa^{\prime}(y-x)^{2}+\kappa(y-x)^{2} \mathrm{~d} x \mathrm{~d} y \\
& =2 \int_{0}^{R}\left(\int_{-x}^{R-x} \kappa^{\prime \prime}(z)^{2}-2 \kappa^{\prime}(z)^{2}+\kappa(z)^{2} \mathrm{~d} z\right) \mathrm{d} x \\
& =2 R \int_{0}^{1}\left(\int_{-R x}^{R(1-x)} \kappa^{\prime \prime}(z)^{2}-2 \kappa^{\prime}(z)^{2}+\kappa(z)^{2} \mathrm{~d} z\right) \mathrm{d} x .
\end{aligned}
$$

Integrating by parts, we have:

$$
\int_{-R x}^{R(1-x)} \kappa^{\prime}(z)^{2} \mathrm{~d} z=\kappa(R(1-x)) \kappa^{\prime}(R(1-x))-\kappa(-R x) \kappa^{\prime}(-R x)-\int_{-R x}^{R(1-x)} \kappa(z) \kappa(z)^{\prime \prime} \mathrm{d} x,
$$

so that

$$
\begin{aligned}
\int_{-R x}^{R(1-x)} \kappa^{\prime \prime}(z)^{2}-2 \kappa^{\prime}(z)^{2}+\kappa(z)^{2} \mathrm{~d} z= & \int_{-R x}^{R(1-x)}\left(\kappa(z)+\kappa^{\prime \prime}(z)\right)^{2} \mathrm{~d} z \\
& +\kappa(R(1-x)) \kappa^{\prime}(R(1-x))-\kappa(-R x) \kappa^{\prime}(-R x) .
\end{aligned}
$$

Recall that $\kappa \kappa^{\prime}$ tends to 0 at infinity and that $\kappa$ is even. Letting $R \rightarrow+\infty$ in the previous equation, we obtain for any $x \in(0,1)$ :

$$
\int_{-R x}^{R(1-x)} \kappa^{\prime \prime}(z)^{2}-2 \kappa^{\prime}(z)^{2}+\kappa(z)^{2} \mathrm{~d} z \underset{R \rightarrow+\infty}{\longrightarrow} \int_{-\infty}^{+\infty}\left(\kappa(z)+\kappa^{\prime \prime}(z)\right)^{2} \mathrm{~d} z
$$

where the right-hand side is finite since both $\kappa$ and $\kappa^{\prime \prime}$ are square-integrable. By Lebesgue's Dominated Convergence Theorem, we get:

$$
\frac{1}{R} \operatorname{Var}\left(\int_{0}^{R} f^{\prime}(x)^{2}-f(x)^{2} \mathrm{~d} x\right) \underset{R \rightarrow+\infty}{\longrightarrow} 4 \int_{0}^{+\infty}\left(\kappa(z)+\kappa^{\prime \prime}(z)\right)^{2} \mathrm{~d} z .
$$

In this last step the dominating function is constant on $[0,1]$ equal to:

$$
2\|\kappa\|_{1}^{2}+\int_{-\infty}^{+\infty}\left(\kappa(z)+\kappa^{\prime \prime}(z)\right)^{2} \mathrm{~d} z
$$

The following corollary proves the positivity of $\sigma^{2}$ and concludes the proof of Proposition 1.11 .

Corollary 4.8 (Explicit lower bound on $\sigma^{2}$ ). Let $f$ be a normalized centered stationary centered Gaussian $\mathcal{C}^{2}$-process and let $\kappa$ denote its correlation function. Under the hypotheses of Proposition 1.11, the constant $\sigma^{2}$ defined by Equation (1.2) satisfies:

$$
\sigma^{2} \geqslant \frac{1}{\pi^{2}} \int_{0}^{+\infty}\left(\kappa(z)+\kappa^{\prime \prime}(z)\right)^{2} \mathrm{~d} z>0 .
$$

Proof. Let $Z$ denote the zero set of $f$ and let $\nu$ denote its counting measure, as in Section 2.3. As we already said, we have: $\operatorname{Card}(Z \cap[0, R])=\left\langle\nu, \mathbb{1}_{[0, R]}\right\rangle=\left\langle\nu_{R}, \mathbb{1}_{[0,1]}\right\rangle$. We use the asymptotics given by Equation (1.1) with $\phi_{1}=\phi_{2}=\mathbb{1}_{[0,1]}$. Note that this asymptotics was already proved to hold, in Section 4.1. Then, as $R \rightarrow+\infty$,

$$
m_{2}\left(\nu_{R}\right)\left(\mathbb{1}_{[0,1]}, \mathbb{1}_{[0,1]}\right)=\operatorname{Var}\left(\left\langle\nu_{R}, \mathbb{1}_{[0,1]}\right\rangle\right)=R \sigma^{2}+o(R) .
$$


That is, $\frac{1}{R} \operatorname{Var}(\operatorname{Card}(Z \cap[0, R])) \underset{R \rightarrow+\infty}{\longrightarrow} \sigma^{2}$.

By Proposition 4.5, we have:

$$
\operatorname{Var}(\operatorname{Card}(Z \cap[0, R])) \geqslant \frac{1}{4 \pi^{2}} \operatorname{Var}\left(\int_{0}^{R} f^{\prime}(x)^{2}-f(x)^{2} \mathrm{~d} x\right) .
$$

We divide by $R$ and let $R \rightarrow+\infty$. By Lemma 4.7, we have:

$$
\sigma^{2}=\lim _{R \rightarrow+\infty} \frac{1}{R} \operatorname{Var}(\operatorname{Card}(Z \cap[0, R])) \geqslant \frac{1}{\pi^{2}} \int_{0}^{+\infty}\left(\kappa(z)+\kappa^{\prime \prime}(z)\right)^{2} \mathrm{~d} z .
$$

In order to conclude the proof, we need to check that the right-hand side of the previous equation is positive. It is clearly non-negative. If it were zero, then $\kappa$ would be an even function of class $\mathcal{C}^{2}$ such that $\kappa(0)=1, \kappa^{\prime}(0)=0$ and $\forall z \geqslant 0, \kappa(z)+\kappa^{\prime \prime}(z)=0$. That is we would have $\kappa(z)=\cos (z)$ for all $z \in \mathbb{R}$. This would contradict our hypotheses on $\kappa$, for example the fact that $\kappa(z) \underset{z \rightarrow+\infty}{\longrightarrow} 0$. Thus,

$$
\int_{0}^{+\infty}\left(\kappa(z)+\kappa^{\prime \prime}(z)\right)^{2} \mathrm{~d} z>0
$$

\section{Divided differences}

In this section, we introduce another important tool that we will use in the proofs of Theorems 1.6, 1.13 and 1.14: the divided differences. The divided differences associated with a point $x \in \mathbb{R}^{p}$ and a function $f \in \mathcal{C}^{p}(\mathbb{R})$ are coefficients of the Hermite interpolation polynomial of $f$ at $x$ (see Definition 5.6 below). As such, they are an important object in polynomial approximation and are well-studied. In Section 5.1, we define the divided differences and the related Hermite interpolation polynomials. In Section 5.2, we state the properties of the divided differences that we are interested in. Most of the material of these two sections is classical and can be found in the survey [19]. Finally, in Section 5.3, we study the distribution of the divided differences associated with a stationary centered Gaussian process.

\subsection{Hermite interpolation and divided differences}

The goal of this section is to define the so-called divided differences associated with a point $x \in \mathbb{R}^{p}$ and a function $f \in \mathcal{C}^{p-1}(\mathbb{R})$. First we define the evaluation at $x \in \mathbb{R}^{p}$ and introduce some useful notations. Then we define the Hermite interpolation polynomial of $f$ at $x$ and the associated divided differences in Definition 5.6.

Definition 5.1 (Evaluation map). Let $p \in \mathbb{N}^{*}$ and let $x=\left(x_{i}\right)_{1 \leqslant i \leqslant p} \in \mathbb{R}^{p}$. For all $i \in\{1, \ldots, p\}$ we denote by $c_{i}(x)=\operatorname{Card}\left\{j \in\{1, \ldots, i-1\} \mid x_{j}=x_{i}\right\}$. We denote by $\mathrm{ev}_{x}: \mathcal{C}^{p-1}(\mathbb{R}) \rightarrow \mathbb{R}^{p}$ the evaluation map defined by:

$$
\mathrm{ev}_{x}: f \longmapsto\left(\frac{f^{\left(c_{i}(x)\right)}\left(x_{i}\right)}{c_{i}(x) !}\right)_{1 \leqslant i \leqslant p} .
$$

Example 5.2. If $x=\left(x_{i}\right)_{1 \leqslant i \leqslant p} \in \mathbb{R}^{p} \backslash \Delta_{p}$, then $\mathrm{ev}_{x}: f \mapsto\left(f\left(x_{1}\right), \ldots, f\left(x_{p}\right)\right)$ is the classical evaluation map at the points $\left(x_{i}\right)_{1 \leqslant i \leqslant p}$. On the diagonal, we also evaluate derivatives of $f:$ if $x=\left(y_{1}, \ldots, y_{1}, \ldots, y_{m}, \ldots, y_{m}\right)$, where the $\left(y_{j}\right)_{1 \leqslant j \leqslant m}$ are distinct and $y_{j}$ is repeated $k_{j}+1$ times, then $p=\sum_{j=1}^{m}\left(k_{j}+1\right)$ and

$$
\mathrm{ev}_{x}: f \longmapsto\left(f\left(y_{1}\right), f^{\prime}\left(y_{1}\right), \ldots, \frac{f^{\left(k_{1}\right)}\left(y_{1}\right)}{k_{1} !}, \ldots, f\left(y_{m}\right), f^{\prime}\left(y_{m}\right), \ldots, \frac{f^{\left(k_{m}\right)}\left(y_{m}\right)}{k_{m} !}\right)
$$


More generally, with the notations of Section 2.1, let $\mathcal{I} \in \mathcal{P}_{p}$, let $y=\left(y_{I}\right)_{I \in \mathcal{I}} \in \mathbb{R}^{\mathcal{I}} \backslash \Delta_{\mathcal{I}}$ and let $x=\iota_{\mathcal{I}}(y) \in \Delta_{p, \mathcal{I}}$. Then, for any $f \in \mathcal{C}^{p-1}(\mathbb{R})$, we have:

$$
\operatorname{ev}_{x}(f)=\left(\frac{f^{(i)}\left(y_{I}\right)}{i !}\right)_{I \in \mathcal{I}, 0 \leqslant i<|I|} .
$$

Definitions 5.3 (Newton polynomials). Let $p \in \mathbb{N}^{*}$ and let $x=\left(x_{i}\right)_{1 \leqslant i \leqslant p} \in \mathbb{R}^{p}$.

- We denote by $\mathbb{R}_{p-1}[X]$ the space of polynomials in $X$ of degree at most $p-1$.

- For all $j \in\{0, \ldots, p-1\}$, we denote by $P_{x}^{j}=\prod_{l=1}^{j}\left(X-x_{l}\right)$ the $j$-th Newton polynomial associated with $x$.

- Let $M(x)$ denote the matrix of the restriction of $\mathrm{ev}_{x}$ to $\mathbb{R}_{p-1}[X]$, in the basis $\left(P_{x}^{0}, \ldots, P_{x}^{p-1}\right)$ of $\mathbb{R}_{p-1}[X]$ and the canonical basis of $\mathbb{R}^{p}$ (see Example 5.10 .1 below).

Lemma 5.4. Let $p \in \mathbb{N}^{*}$ and let $x=\left(x_{i}\right)_{1 \leqslant i \leqslant p} \in \mathbb{R}^{p}$. The matrix $M(x)=\left(M_{i j}(x)\right)_{1, \leqslant i, j \leqslant p}$ is lower triangular and, for all $i \in\{1, \ldots, p\}$, we have:

$$
M_{i i}(x)=\prod_{\left\{k \in\{1, \ldots, i-1\} \mid x_{k} \neq x_{i}\right\}}\left(x_{i}-x_{k}\right) .
$$

Moreover, if $1 \leqslant j<i \leqslant p$, the coefficient $M_{i j}(x)$ vanishes when $c_{i}(x) \geqslant j$ (cf. Definition 5.1), and is an homogeneous polynomial of degree $j-1-c_{i}(x)$ in $\left(x_{i}-x_{l}\right)_{1 \leqslant l<j}$ when $c_{i}(x)<j$.

Proof. Let $x=\left(x_{i}\right)_{1 \leqslant i \leqslant p} \in \mathbb{R}^{p}$ and let $i, j \in\{1, \ldots, p\}$. By definition of $M(x)$, we have

$$
M_{i j}(x)=\frac{\left(P_{x}^{j-1}\right)^{\left(c_{i}(x)\right)}\left(x_{i}\right)}{c_{i}(x) !} .
$$

If $i<j$, then Card $\left\{l<j \mid x_{l}=x_{i}\right\} \geqslant c_{i}(x)+1$. Hence, $x_{i}$ is a root of $P_{x}^{j-1}$ of multiplicity at least $c_{i}(x)+1$, and $\left(P_{x}^{j-1}\right)^{\left(c_{i}(x)\right)}\left(x_{i}\right)=0$. Thus $M(x)$ is lower triangular. Then, if $i=j$, we have

$$
P_{x}^{j-1}=\left(X-x_{i}\right)^{c_{i}(x)} \prod_{k \in K}\left(X-x_{k}\right),
$$

where $K=\left\{k \in\{1, \ldots, i-1\} \mid x_{k} \neq x_{i}\right\}$. Hence, $M_{i i}(x)=\prod_{k \in K}\left(x_{i}-x_{k}\right)$ as claimed.

Let us now assume that $j<i$. If $c_{i}(x) \geqslant j$, since $P_{x}^{j-1}$ has degree $j-1$ we have $\left(P_{x}^{j-1}\right)^{\left(c_{i}(x)\right)}=0$, and $M_{i j}(x)=0$. If $c_{i}(x)<j$, then $\left(P_{x}^{j-1}\right)^{\left(c_{i}(x)\right)}$ is a sum of terms which are products of exactly $j-1-c_{i}(x)$ factors of the form $\left(X-x_{l}\right)$, where $1 \leqslant l<j$. Thus $M_{i j}(x)$ is some homogeneous polynomial of degree $j-1-c_{i}(x)$ evaluated on $\left(x_{i}-x_{l}\right)_{1 \leqslant l<j}$.

Corollary 5.5. For all $x \in \mathbb{R}^{p}$, the restriction of $\mathrm{ev}_{x}$ is an isomorphism from $\mathbb{R}_{p-1}[X]$ to $\mathbb{R}^{p}$.

Proof. By Lemma 5.4, the matrix $M(x)$ of this linear map is lower triangular and its diagonal coefficients are non-zero.

We can now define the Hermite interpolation polynomial of $f$ at $x \in \mathbb{R}^{p}$ and the divided difference $[f]_{p}(x)$. The meaning of the name "divided difference" is not obvious in the following definition. The terminology will become clearer after we explained how to compute these divided differences recursively (see Lemma 5.12 below).

Definition 5.6 (Divided differences). Let $p \in \mathbb{N}^{*}$ and let $x \in \mathbb{R}^{p}$. By Corollary 5.5, for any $f \in \mathcal{C}^{p-1}(\mathbb{R})$ there exists a unique $\pi_{x}^{f} \in \mathbb{R}_{p-1}[X]$ such that $\operatorname{ev}_{x}\left(\pi_{x}^{f}\right)=\operatorname{ev}_{x}(f)$. This polynomial is called the Hermite interpolation polynomial of $f$ at $x$. The divided difference $[f]_{p}(x)$ is defined as its leading coefficient. 
The following lemma shows that the divided differences are the coordinates of the Hermite interpolation polynomial in the basis of the Newton polynomials defined above, see Definitions 5.3.

Lemma 5.7. Let $p \in \mathbb{N}^{*}$ and let $x=\left(x_{i}\right)_{1 \leqslant i \leqslant p} \in \mathbb{R}^{p}$. For all $f \in \mathcal{C}^{p-1}(\mathbb{R})$, we have:

$$
\pi_{x}^{f}=\sum_{j=1}^{p}[f]_{j}\left(x_{1}, \ldots, x_{j}\right) P_{x}^{j-1} .
$$

Proof. We prove this result by induction on $p \in \mathbb{N}^{*}$. If $p=1$, for any continuous $f$, the polynomial $\pi_{x}^{f}$ is constant equal to $f\left(x_{1}\right)$. Hence, $\pi_{x}^{f}=[f]_{1}\left(x_{1}\right) P_{x}^{0}$ where $[f]_{1}=f$.

Let us assume that the result holds for $p \in \mathbb{N}^{*}$. Let $x=\left(x_{i}\right)_{1 \leqslant i \leqslant p+1} \in \mathbb{R}^{p+1}$, we denote by $\widetilde{x}=\left(x_{i}\right)_{1 \leqslant i \leqslant p}$. Note that for any $f \in \mathcal{C}^{p}(\mathbb{R})$, the components of $\operatorname{ev}_{\widetilde{x}}(f)$ are the first $p$ components of $\operatorname{ev}_{x}(f)$. Then, by Lemma 5.4, we have $\operatorname{ev}_{\widetilde{x}}\left(P_{x}^{p}\right)=0$. Hence,

$$
\operatorname{ev}_{\widetilde{x}}\left(\pi_{x}^{f}-[f]_{p+1}(x) P_{x}^{p}\right)=\operatorname{ev}_{\widetilde{x}}\left(\pi_{x}^{f}\right)=\operatorname{ev}_{\widetilde{x}}(f) .
$$

Moreover, by Definition 5.6, the polynomial $\pi_{x}^{f}-[f]_{p+1}(x) P_{x}^{p}$ has degree at most $p-1$. Thus, $\pi_{x}^{f}-[f]_{p+1}(x) P_{x}^{p}=\pi_{\widetilde{x}}^{f}=\sum_{j=1}^{p}[f]_{j}\left(x_{1}, \ldots, x_{j}\right) P_{x}^{j-1}$, where the second equality is given by the induction hypothesis. This concludes the induction step and the proof.

Definition 5.8 (Divided differences evaluation map). Let $p \in \mathbb{N}^{*}$ and $x=\left(x_{i}\right)_{1 \leqslant i \leqslant p} \in \mathbb{R}^{p}$, we denote by $[\mathrm{ev}]_{x}: \mathcal{C}^{p-1}(\mathbb{R}) \rightarrow \mathbb{R}^{p}$ the linear map defined by

$$
[\mathrm{ev}]_{x}: f \longmapsto\left([f]_{j}\left(x_{1}, \ldots, x_{j}\right)\right)_{1 \leqslant j \leqslant p} .
$$

Lemma 5.9. Let $p \in \mathbb{N}^{*}$, for all $x \in \mathbb{R}^{p}$ we have $M(x)[\mathrm{ev}]_{x}=\mathrm{ev}_{x}$, where $\mathrm{ev}_{x}$ is as in Definition 5.1 and $M(x)$ is defined by Definitions 5.3.

Proof. Let $x \in \mathbb{R}^{p}$ and let $f \in \mathcal{C}^{p-1}(\mathbb{R})$. By Lemma 5.7, the components of $[\mathrm{ev}]_{x}(f)$ are the coordinates of the polynomial $\pi_{x}^{f}$ in the basis $\left(P_{x}^{j}\right)_{1 \leqslant j \leqslant p}$ of $\mathbb{R}_{p-1}[X]$. Then, by definition of $M(x)$ and $\pi_{x}^{f}$, we have:

$$
M(x)[\mathrm{ev}]_{x}(f)=\mathrm{ev}_{x}\left(\pi_{x}^{f}\right)=\mathrm{ev}_{x}(f)
$$

Example 5.10. We conclude this section by giving some examples.

1. Let $f \in \mathcal{C}^{1}(\mathbb{R})$ and $\left(x_{1}, x_{2}\right) \in \mathbb{R}^{2} \backslash \Delta_{2}$, we have $M(x)=\left(\begin{array}{cc}1 & 0 \\ 1 & x_{2}-x_{1}\end{array}\right)$. Hence

$$
\left(\begin{array}{c}
{[f]_{1}\left(x_{1}\right)} \\
{[f]_{2}\left(x_{1}, x_{2}\right)}
\end{array}\right)=M(x)^{-1}\left(\begin{array}{c}
f\left(x_{1}\right) \\
f\left(x_{2}\right)
\end{array}\right)=\left(\begin{array}{cc}
1 & 0 \\
\frac{-1}{x_{2}-x_{1}} & \frac{1}{x_{2}-x_{1}}
\end{array}\right)\left(\begin{array}{l}
f\left(x_{1}\right) \\
f\left(x_{2}\right)
\end{array}\right)=\left(\begin{array}{c}
f\left(x_{1}\right) \\
\frac{f\left(x_{2}\right)-f\left(x_{1}\right)}{x_{2}-x_{1}}
\end{array}\right) .
$$

2. Let $p \in \mathbb{N}^{*}$, let $x=\left(x_{i}\right)_{1 \leqslant i \leqslant p} \in \mathbb{R}^{p}$ and let $f \in \mathcal{C}^{p-1}(\mathbb{R})$. If there exists $z \in \mathbb{R}$ such that $x_{i}=z$ for all $i \in\{1, \ldots, p\}$, then $\operatorname{ev}_{x}(f)=\left(f(z), \ldots, \frac{f^{(p-1)}(z)}{(p-1) !}\right)$ and $M(x)$ is the identity matrix of size $p$. Then, $\pi_{x}^{f}$ is the Taylor polynomial of degree $p-1$ of $f$ at $z$ and $[f]_{j}\left(x_{1}, \ldots, x_{j}\right)=\frac{f^{(j-1)}(z)}{(j-1) !}$, for all $j \in\{1, \ldots, p\}$.

3. Let $p \in \mathbb{N}^{*}$ and $\left(x_{i}\right)_{1 \leqslant i \leqslant p} \in \mathbb{R}^{p} \backslash \Delta_{p}$. Let $f \in \mathcal{C}^{p}(\mathbb{R})$ be such that $[f]_{j}\left(x_{1}, \ldots, x_{j}\right)=0$ for all $j \in\{1, \ldots, p\}$. Let $i \in\{1, \ldots, p\}$, we denote by $x=\left(x_{1}, \ldots, x_{p}, x_{i}\right) \in \mathbb{R}^{p+1}$. By Lemma 5.7, we have $\pi_{x}^{f}=[f]_{p+1}\left(x_{1}, \ldots, x_{p}, x_{i}\right) \prod_{j=1}^{p}\left(X-x_{j}\right)$. Hence,

$$
f^{\prime}\left(x_{i}\right)=\left(\pi_{x}^{f}\right)^{\prime}\left(x_{i}\right)=[f]_{p+1}\left(x_{1}, \ldots, x_{p}, x_{i}\right) \prod_{j \in\{1, \ldots, p\} \backslash\{i\}}\left(x_{i}-x_{j}\right) .
$$




\subsection{Properties of the divided differences}

Let us now derive some interesting properties of the divided differences defined in Definition 5.6. They will be useful in Section 6, to obtain new expressions of the Kac-Rice densities (cf. Definition 3.1) and prove clustering results for these densities.

Recall that we denoted by $\sigma \cdot x$ the action of $\sigma \in \mathfrak{S}_{p}$ on $x \in \mathbb{R}^{p}$ by permutation of the indices (see Notation 3.4).

Lemma 5.11 (Symmetry). Let $p \in \mathbb{N}^{*}$ and $f \in \mathcal{C}^{p-1}(\mathbb{R})$. For all $x \in \mathbb{R}^{p}$ and all $\sigma \in \mathfrak{S}_{p}$, we have $\pi_{\sigma \cdot x}^{f}=\pi_{x}^{f}$. In particular $[f]_{p}(\sigma \cdot x)=[f]_{p}(x)$, that is the function $[f]_{p}: \mathbb{R}^{p} \rightarrow \mathbb{R}$ is symmetric.

Proof. Let $x \in \mathbb{R}^{p}$. By Remark 2.4, there exists a unique $\mathcal{I} \in \mathcal{P}_{p}$ such that $x \in \Delta_{p, \mathcal{I}}$. Moreover, there exists a unique $y=\left(y_{I}\right)_{I \in \mathcal{I}} \in \mathbb{R}^{\mathcal{I}} \backslash \Delta_{\mathcal{I}}$ such that $x=\iota_{\mathcal{I}}(y)$. By Definition 5.6, the polynomial $\pi_{x}^{f}$ is the only element of $\mathbb{R}_{p-1}[X] \operatorname{such} \operatorname{that}\left(\pi_{x}^{f}-f\right)^{(i)}\left(y_{I}\right)=0$ for all $I \in \mathcal{I}$ and all $i<|I|$. The set $\left\{\left(y_{I},|I|\right) \mid I \in \mathcal{I}\right\}$ is invariant under the action of $\sigma$ on $x$ by permutation of the components. Hence $\pi_{\sigma \cdot x}^{f}=\pi_{x}^{f}$ and, looking at the leading coefficients, we have $[f]_{p}(\sigma \cdot x)=[f]_{p}(x)$.

The following result shows that the divided differences can be computed recursively, at least if the interpolation points $x_{1}, \ldots, x_{p} \in \mathbb{R}$ are distinct. It also explains the name "divided differences".

Lemma 5.12 (Inductive definition). Let $p \in \mathbb{N}^{*}$, let $f \in \mathcal{C}^{p}(\mathbb{R})$ and $x=\left(x_{i}\right)_{1 \leqslant i \leqslant p+1} \in \mathbb{R}^{p+1}$ be such that $x_{p} \neq x_{p+1}$. Then, we have:

$$
[f]_{p+1}(x)=\frac{[f]_{p}\left(x_{1}, \ldots, x_{p-1}, x_{p+1}\right)-[f]_{p}\left(x_{1} \ldots, x_{p-1}, x_{p}\right)}{x_{p+1}-x_{p}} .
$$

Proof. Let $\sigma \in \mathfrak{S}_{p+1}$ be defined by $\sigma(p)=p+1, \sigma(p+1)=p$ and $\sigma(i)=i$ for all $i \in\{1, \ldots, p-1\}$. Using Notation 3.4, by Lemmas 5.7 and 5.11, we have:

$$
0=\pi_{x}^{f}-\pi_{\sigma \cdot x}^{f}=\sum_{j=1}^{p+1}[f]_{j}\left(x_{1}, \ldots, x_{j}\right) P_{x}^{j-1}-\sum_{j=1}^{p+1}[f]_{j}\left(x_{\sigma(1)}, \ldots, x_{\sigma(j)}\right) P_{\sigma \cdot x}^{j-1} .
$$

We have $P_{\sigma \cdot x}^{j-1}=P_{x}^{j-1}$ for $j \in\{1, \ldots, p\}$. Moreover, $[f]_{j}\left(x_{\sigma(1)}, \ldots, x_{\sigma(j)}\right)=[f]_{j}\left(x_{1} \ldots, x_{j}\right)$ for all $j \in\{1, \ldots, p+1\} \backslash\{p\}$. Hence, only the terms of index $p$ and $p+1$ do not cancel out in the previous sums. Dividing by $P_{x}^{p-1}=\prod_{i=1}^{p-1}\left(X-x_{i}\right)=P_{\sigma \cdot x}^{p-1}$, we obtain:

$$
\left(x_{p+1}-x_{p}\right)[f]_{p+1}\left(x_{1}, \ldots, x_{p}, x_{p+1}\right)+[f]_{p}\left(x_{1}, \ldots, x_{p-1}, x_{p+1}\right)-[f]_{p}\left(x_{1} \ldots, x_{p-1}, x_{p}\right)=0 .
$$

Notation 5.13. Let $p \in \mathbb{N}^{*}$ and $x=\left(x_{i}\right)_{1 \leqslant i \leqslant p}$, we denote by $x_{\min }=\min \left\{x_{i} \mid 1 \leqslant i \leqslant p\right\}$ and by $x_{\max }=\max \left\{x_{i} \mid 1 \leqslant i \leqslant p\right\}$.

Lemma 5.14 (Rolle's Property). Let $p \in \mathbb{N}^{*}$ and $f \in \mathcal{C}^{p-1}(\mathbb{R})$. For all $x=\left(x_{i}\right)_{1 \leqslant i \leqslant p} \in \mathbb{R}^{p}$, there exists $\xi \in\left[x_{\min }, x_{\max }\right]$ such that $[f]_{p}(x)=\frac{f^{(p-1)}(\xi)}{(p-1) !}$.

Proof. Let $x \in \mathbb{R}^{p}$. There exist $y_{1}<\cdots<y_{m}$ and $k_{1}, \ldots, k_{m} \in \mathbb{N}$ such that, for all $j \in\{1, \ldots, m\}$, exactly $k_{j}+1$ components of $x$ are equal to $y_{j}$. With these notations, $x_{\min }=y_{1}$ and $x_{\max }=y_{m}$. By Definition 5.6, $\left(f-\pi_{x}^{f}\right)$ has at least $p$ zeros in $\left[x_{\min }, x_{\max }\right]$, counted with multiplicity. More precisely, $\forall j \in\{1, \ldots, m\}, \forall k \in\left\{0, \ldots, k_{j}\right\}$, we have $\left(f-\pi_{x}^{f}\right)^{(k)}\left(y_{j}\right)=0$.

For all $j \in\{1, \ldots, m-1\}$, there exists $z_{j} \in\left(y_{j}, y_{j+1}\right)$ such that $\left(f-\pi_{x}^{f}\right)^{\prime}\left(z_{j}\right)=0$, by Rolle's Theorem. Hence $\left(f-\pi_{x}^{f}\right)^{\prime}$ has at least $p-1$ zeros in $\left[x_{\min }, x_{\max }\right]$, namely $z_{1}, \ldots, z_{m-1}$ with multiplicity 1 , and $y_{j}$ with multiplicity $k_{j}-1$, for all $j \in\{1, \ldots, m\}$. Iterating this procedure, for all $k \in\{0, \ldots, p-1\}$, the function $\left(f-\pi_{x}^{f}\right)^{(k)}$ has at least $p-k$ 
zeros in $\left[x_{\min }, x_{\max }\right]$, counted with multiplicity. In particular, there exists $\xi \in\left[x_{\min }, x_{\max }\right]$ such that:

$$
\left(f-\pi_{x}^{f}\right)^{(p-1)}(\xi)=f^{(p-1)}(\xi)-(p-1) ![f]_{p}(x)=0 .
$$

Lemma 5.15 (Continuity). If $f \in \mathcal{C}^{p-1}(\mathbb{R})$, then the function $[f]_{p}: \mathbb{R}^{p} \rightarrow \mathbb{R}$ is continuous.

Proof. We prove this result by induction on $p$. If $p=1$, then $[f]_{1}=f$ is continuous on $\mathbb{R}$.

Let us now that the result holds for some $p \in \mathbb{N}^{*}$ and let $f \in \mathcal{C}^{p}(\mathbb{R})$. Using Lemma 5.12 and the induction hypothesis, $[f]_{p+1}$ is continuous on $\left\{\left(x_{1}, \ldots, x_{p+1}\right) \in \mathbb{R}^{p+1} \mid x_{p} \neq x_{p+1}\right\}$. By symmetry (see Lemma 5.11), this map is in fact continuous at any point $\left(x_{i}\right)_{1 \leqslant i \leqslant p+1}$ such that $x_{i} \neq x_{j}$ for some $i, j \in\{1, \ldots, p+1\}$. In order to conclude the proof, it is enough to prove that, for all $z \in \mathbb{R}$,

$$
[f]_{p+1}(x) \underset{x \rightarrow(z, z, \ldots, z)}{\longrightarrow}[f]_{p+1}(z, \ldots, z)
$$

We have seen in Example 5.10.2 that $[f]_{p+1}(z, \ldots, z)=\frac{f^{(p)}(z)}{p !}$. Let $x \in \mathbb{R}^{p+1}$, by Lemma 5.14, there exists $\xi \in\left[x_{\min }, x_{\max }\right]$ such that $[f]_{p+1}(x)=\frac{f^{(p)}(\xi)}{p !}$. As $x \rightarrow(z, \ldots, z)$, we have $x_{\min } \rightarrow z$ and $x_{\max } \rightarrow z$. The conclusion follows from the continuity of $f^{(p)}$.

Remark 5.16. Let $p \in \mathbb{N}^{*}$ and $f \in \mathcal{C}^{p}(\mathbb{R})$, for all $x=\left(x_{i}\right)_{1 \leqslant i \leqslant p+1} \in \mathbb{R}^{p}$ we have:

$$
[f]_{p+1}(x)=\lim _{z \rightarrow x_{p+1}} \frac{[f]_{p}\left(x_{1}, \ldots, x_{p-1}, z\right)-[f]_{p}\left(x_{1}, \ldots, x_{p-1}, x_{p}\right)}{z-x_{p}} .
$$

If $x_{p} \neq x_{p+1}$, this is follows from Lemma 5.12 and the continuity of $[f]_{p}$ (see Lemma 5.15). If $x_{p}=x_{p+1}$ this follows from the first case and the continuity of $[f]_{p+1}$. Thus, one can define the divided differences recursively as follows: if $f \in \mathcal{C}^{0}(\mathbb{R})$ then $[f]_{1}=f$, and if $f \in \mathcal{C}^{p}(\mathbb{R})$ the map $[f]_{p+1}: \mathbb{R}^{p+1} \rightarrow \mathbb{R}$ is defined by Equation (5.1). This definition is equivalent to Definition 5.6.

Lemma 5.17 (Regularity). Let $p \in \mathbb{N}^{*}$ and let $k \in \mathbb{N}$, if $f \in \mathcal{C}^{p+k-1}(\mathbb{R})$ then $[f]_{p}: \mathbb{R}^{p} \rightarrow \mathbb{R}$ is of class $\mathcal{C}^{k}$. Moreover, for all $k_{1}, \ldots, k_{p} \in \mathbb{N}$ such that $k_{1}+\cdots+k_{p} \leqslant k$, for all $x=\left(x_{i}\right)_{1 \leqslant i \leqslant p} \in \mathbb{R}^{p}$, we have:

$$
\frac{1}{k_{1} ! \ldots k_{p} !} \frac{\partial^{k_{1}+\cdots+k_{p}}[f]_{p}}{\partial x_{1}^{k_{1}} \ldots \partial x_{p}^{k_{p}}}(x)=[f]_{p+k_{1}+\cdots+k_{p}}\left(x_{1}, \ldots, x_{1}, \ldots, x_{p}, \ldots, x_{p}\right),
$$

where each $x_{j}$ is repeated $k_{j}+1$ times on the right-hand side.

Proof. We prove this result by induction on $k$. The case $k=0$ is given by Lemma 5.15.

For $k=1$, let $p \in \mathbb{N}^{*}$ and let $f \in \mathcal{C}^{p}(\mathbb{R})$. By Lemmas 5.12 and 5.15 (see also Remark 5.16, Equation (5.1)), the map $[f]_{p}$ admits a continuous partial derivative with respect to the $p$-th variable, given by:

$$
\frac{\partial[f]_{p}}{\partial x_{p}}:\left(x_{1}, \ldots, x_{p}\right) \mapsto[f]_{p+1}\left(x_{1}, \ldots, x_{p}, x_{p}\right) .
$$

The symmetry of the divided differences (see Lemma 5.11) yields that $[f]_{p}$ is of class $\mathcal{C}^{1}$, with partial derivatives given by Equation (5.2).

Let $k \in \mathbb{N}^{*}$ and let us assume that the result holds for $k$ and any $p \in \mathbb{N}^{*}$. Let $p \in \mathbb{N}^{*}$ and let $f \in \mathcal{C}^{p+k}(\mathbb{R})$. Using the case $k=1$, the map $[f]_{p}$ is $\mathcal{C}^{1}$ and its partial derivatives of order 1 are given by Equation (5.2). The induction hypothesis shows that $[f]_{p+1}$ is of class $\mathcal{C}^{k}$, hence $[f]_{p}$ is of class $\mathcal{C}^{k+1}$. The induction hypothesis also shows that the partial 
derivatives of order at most $k$ of $[f]_{p}$ are given by (5.2). Let $k_{1}, \ldots, k_{p} \in \mathbb{N}$ be such that $k_{1}+\cdots+k_{p}=k$ and let $i \in\{1, \ldots, p\}$. We have:

$$
\frac{\partial}{\partial x_{i}}\left(\frac{1}{k_{1} ! \ldots k_{p} !} \frac{\partial^{k_{1}+\cdots+k_{p}}[f]_{p}}{\partial x_{1}^{k_{1}} \ldots \partial x_{p}^{k_{p}}}\right)=\frac{\partial}{\partial x_{i}}\left(x \mapsto[f]_{p+k}\left(x_{1}, \ldots, x_{1}, \ldots, x_{p}, \ldots, x_{p}\right)\right),
$$

where each $x_{j}$ is repeated $k_{j}+1$ times on the right-hand side. Using the case $k=1$ for $[f]_{p+k}$ proves that the partial derivatives of order $k+1$ of $[f]_{p}$ satisfy Equation (5.2).

We conclude this section by stating facts that provide some insight on divided differences. Let $p \in \mathbb{N}^{*}$ and let $x=\left(x_{i}\right)_{1 \leqslant i \leqslant p} \in \mathbb{R}^{p} \backslash \Delta_{p}$, for all $f \in \mathcal{C}^{p-1}(\mathbb{R})$, we have:

$$
[f]_{p}(x)=\sum_{i=1}^{p} f\left(x_{i}\right) \prod_{l \in\{1, \ldots, p\} \backslash\{i\}} \frac{1}{x_{i}-x_{l}}
$$

This formula is proved by induction on $p \in \mathbb{N}^{*}$, using Lemma 5.12 in the induction step. Taking partial derivatives in the previous formula and using Lemma 5.17 allows to derive an expression of $[f]_{p}(x)$ for any $p \in \mathbb{N}^{*}$, any $x=\left(x_{i}\right)_{1 \leqslant i \leqslant p} \in \mathbb{R}^{p}$ and any $f \in \mathcal{C}^{p-1}(\mathbb{R})$. One obtains that $[f]_{p}(x)$ is a linear combination of the $f^{(k)}\left(x_{i}\right)$ with $i \in\{1, \ldots, p\}$ and $k<\operatorname{Card}\left\{j \in\{1, \ldots, p\} \mid x_{j}=x_{i}\right\}$. The coefficients of this linear combination are rational functions in $\left(x_{i}-x_{j}\right)_{1 \leqslant j<i \leqslant p}$, independent of $f$. This can already be deduced from the fact that $[f]_{p}(x)$ is the last coordinate of $M(x)^{-1} \mathrm{ev}_{x}(f)$ and the expression of $M(x)$ (see Definitions 5.3 and Lemmas 5.4 and 5.9).

\subsection{Double divided differences and correlation function}

In the previous two sections, we defined and studied the divided differences of some regular enough function. The upshot is to consider the divided differences of the Gaussian process $f$ that we are interested in. Since the evaluation $[\mathrm{ev}]_{x}$ is linear, $[\mathrm{ev}]_{x}(f)$ is a centered Gaussian vector. The goal of this section is to compute and study its variance.

Let $K: \mathbb{R}^{2} \rightarrow \mathbb{R}$ denote the correlation kernel of $f$. In order to compute the coefficients of the variance matrix of $[\mathrm{ev}]_{x}(f)$, we need to take divided differences of $K$ with respect to the first variable, then take divided differences of the result with respect to the second variable. If $f$ was not stationary, this would require to develop a notion of "partial divided differences" and prove parametric versions of the regularity results of Section 5.2. This can be done but is a bit cumbersome. Since we consider stationary processes in this paper, we can avoid these complications and only consider divided differences associated with the correlation function $\kappa: x \mapsto K(0, x)$. We need however to introduce some additional notations.

Let $\kappa: \mathbb{R} \rightarrow \mathbb{R}$ and let $K: \mathbb{R}^{2} \rightarrow \mathbb{R}$ be defined by $K:(z, w) \mapsto \kappa(w-z)$. If $\kappa$ is $\mathcal{C}^{p-1}$ then, for all $y \in \mathbb{R}$, the map $K(\cdot, y): z \mapsto K(z, y)$ is of class $\mathcal{C}^{p-1}$. In particular, the divided differences $[K(\cdot, y)]_{k}$ are well-defined for all $k \in\{1, \ldots, p\}$.

Definition 5.18. Let $p \in \mathbb{N}^{*}$, let $k \in\{1, \ldots, p\}$ and let $\kappa \in \mathcal{C}^{p-1}(\mathbb{R})$. For all $x \in \mathbb{R}^{k}$ and all $y \in \mathbb{R}$, we denote by $[\kappa]_{(k, 1)}(x, y)=[K(\cdot, y)]_{k}(x)$, where $K:(z, w) \mapsto \kappa(w-z)$.

Lemma 5.19. Let $p \in \mathbb{N}^{*}$ and $\kappa \in \mathcal{C}^{p-1}(\mathbb{R})$. Let $k \in\{1, \ldots, p\}$, for all $x=\left(x_{i}\right)_{1 \leqslant i \leqslant k} \in \mathbb{R}^{k}$, for all $y \in \mathbb{R}$, we have:

$$
[\kappa]_{(k, 1)}(x, y)=(-1)^{k-1}[\kappa]_{k}\left(y-x_{1}, \ldots, y-x_{k}\right) .
$$

Proof. Let $K:(z, w) \mapsto \kappa(w-z)$. By Definition 5.6, $[\kappa]_{(k, 1)}(x, y)=[K(\cdot, y)]_{k}(x)$ is the leading coefficient of $\pi_{x}^{K(\cdot, y)}$. Now, recalling Definition 5.1, we have:

$$
\operatorname{ev}_{x}\left(\pi_{x}^{K(\cdot, y)}\right)=\operatorname{ev}_{x}(K(\cdot, y))=\left(\frac{1}{c_{i}(x) !} \frac{\partial^{c_{i}(x)} K}{\partial x^{c_{i}(x)}}\left(x_{i}, y\right)\right)_{1 \leqslant i \leqslant k}=\left((-1)^{c_{i}(x)} \frac{\kappa^{\left(c_{i}(x)\right)}\left(y-x_{i}\right)}{c_{i}(x) !}\right)_{1 \leqslant i \leqslant k},
$$


and $\pi_{x}^{K(\cdot, y)}$ is the only polynomial in $\mathbb{R}_{k-1}[X]$ satisfying this condition. On the other hand, let us denote by $y-x=\left(y-x_{1}, \ldots, y-x_{k}\right) \in \mathbb{R}^{k}$. For all $i \in\{1, \ldots, k\}$, we have $c_{i}(y-x)=c_{i}(x)$. Then, $\pi_{y-x}^{\kappa}(y-X) \in \mathbb{R}_{k-1}[X]$ satisfies:

$\operatorname{ev}_{x}\left(\pi_{y-x}^{\kappa}(y-X)\right)=\left(\frac{(-1)^{c_{i}(x)}}{c_{i}(x) !}\left(\pi_{y-x}^{\kappa}\right)^{\left(c_{i}(x)\right)}\left(y-x_{i}\right)\right)_{1 \leqslant i \leqslant k}=\left((-1)^{c_{i}(x)} \frac{\kappa^{\left(c_{i}(x)\right)}\left(y-x_{i}\right)}{c_{i}(x) !}\right)_{1 \leqslant i \leqslant k}$.

Thus $\pi_{x}^{K(\cdot, y)}=\pi_{y-x}^{\kappa}(y-X)$, and its leading coefficient equals $(-1)^{k-1}[\kappa]_{k}(y-x)$.

A consequence of Lemma 5.19 is that, if $\kappa \in \mathcal{C}^{p-1}(\mathbb{R})$ and $k \in\{1, \ldots, p\}$, then for all $x \in \mathbb{R}^{k}$ the function $[\kappa]_{(k, 1)}(x, \cdot): w \mapsto[\kappa]_{(k, 1)}(x, w)$ is of class $\mathcal{C}^{p-k}$ from $\mathbb{R}$ to $\mathbb{R}$ (see Lemma 5.17). In particular, its the divided differences of order at most $p-k+1$ are well-defined, and the following makes sense.

Definition 5.20 (Double divided differences). Let $p \in \mathbb{N}^{*}$ and let $\kappa \in \mathcal{C}^{p-1}(\mathbb{R})$. Let $k$ and $l \in \mathbb{N}^{*}$ be such that $k+l \leqslant p+1$, we denote by $[\kappa]_{(k, l)}: \mathbb{R}^{k} \times \mathbb{R}^{l} \rightarrow \mathbb{R}$ the map defined by $[\kappa]_{(k, l)}(x, y)=\left[[\kappa]_{(k, 1)}(x, \cdot)\right]_{l}(y)$ for all $x \in \mathbb{R}^{k}$ and $y \in \mathbb{R}^{l}$.

Thanks to Lemma 5.14, we can give bounds on the double divided differences $[\kappa]_{(k, l)}(x, y)$. This is the object of the following result.

Lemma 5.21. Let $k$ and $l \in \mathbb{N}^{*}$ and let $\kappa \in \mathcal{C}^{k+l-2}(\mathbb{R})$, for all $x=\left(x_{i}\right)_{1 \leqslant i \leqslant k} \in \mathbb{R}^{k}$ and all $y=\left(y_{j}\right)_{1 \leqslant j \leqslant l} \in \mathbb{R}^{l}$, we have:

$$
\left|[\kappa]_{(k, l)}(x, y)\right| \leqslant \max \left\{\left|\kappa^{(k+l-2)}(\xi)\right| \mid y_{\min }-x_{\max } \leqslant \xi \leqslant y_{\max }-x_{\min }\right\} .
$$

Proof. Since $[\kappa]_{(k, l)}(x, y)=\left[[\kappa]_{(k, 1)}(x, \cdot)\right]_{l}(y)$, there exists $w_{0} \in\left[y_{\min }, y_{\max }\right]$ such that:

$$
[\kappa]_{(k, l)}(x, y)=\frac{1}{(l-1) !} \frac{\partial^{(l-1)}}{\partial w^{(l-1)}}{ }_{\mid w=w_{0}}[\kappa]_{(k, 1)}(x, w),
$$

by Lemma 5.14. Then, by Lemma 5.19 and Lemma 5.17, we have:

$$
\begin{aligned}
{[\kappa]_{(k, l)}(x, y) } & =\frac{(-1)^{k-1}}{(l-1) !} \frac{\partial^{(l-1)}}{\partial w^{(l-1)}} \mid w=w_{0}[\kappa]_{k}\left(w-x_{1}, \ldots, w-x_{k}\right) \\
& =(-1)^{k-1} \sum_{l_{1}+\cdots+l_{k}=l-1} \frac{1}{l_{1} ! \ldots l_{k} !} \frac{\partial^{l-1}[\kappa]_{k}}{\partial x_{1}^{l_{1}} \ldots \partial x_{k}^{l_{k}}}\left(w_{0}-x_{1}, \ldots, w_{0}-x_{k}\right) \\
& =(-1)^{k-1} \sum_{l_{1}+\cdots+l_{k}=l-1}[\kappa]_{k+l-1}\left(w_{0}-x_{1}, \ldots, w_{0}-x_{1}, \ldots, w_{0}-x_{k}, \ldots, w_{0}-x_{k}\right),
\end{aligned}
$$

where the last two sums are indexed by $\left\{\left(l_{1}, \ldots, l_{k}\right) \in \mathbb{N}^{k} \mid l_{1}+\cdots+l_{k}=l-1\right\}$, and each $w_{0}-x_{i}$ is repeated exactly $l_{i}+1$ times in the term indexed by $\left(l_{1}, \ldots, l_{k}\right)$ on the last line.

Let $\left(l_{1}, \ldots, l_{k}\right) \in \mathbb{N}^{k}$ be such that $l_{1}+\cdots+l_{k}=l-1$. By Lemma 5.14 there exists $\xi_{\left(l_{1}, \ldots, l_{k}\right)} \in \mathbb{R}$ such that:

$$
y_{\min }-x_{\max } \leqslant w_{0}-x_{\max } \leqslant \xi_{\left(l_{1}, \ldots, l_{k}\right)} \leqslant w_{0}-x_{\min } \leqslant y_{\max }-x_{\min },
$$

and

$$
[\kappa]_{k+l-1}\left(w_{0}-x_{1}, \ldots, w_{0}-x_{1}, \ldots, w_{0}-x_{k}, \ldots, w_{0}-x_{k}\right)=\frac{\kappa^{(k+l-2)}\left(\xi_{\left(l_{1}, \ldots, l_{k}\right)}\right)}{(k+l-2) !}
$$

where each term $w_{0}-x_{i}$ is repeated $l_{i}+1$ times on the right-hand side. Thus,

$$
\left|[\kappa]_{(k, l)}(x, y)\right| \leqslant \max \left\{\left|\kappa^{(k+l-2)}(\xi)\right| \mid y_{\min }-x_{\max } \leqslant \xi \leqslant y_{\max }-x_{\min }\right\}
$$

provided that Card $\left\{\left(l_{1}, \ldots, l_{k}\right) \in \mathbb{N}^{k} \mid l_{1}+\cdots+l_{k}=l-1\right\} \leqslant(k+l-2) !$. This cardinal is the dimension of the space of homogeneous polynomials of degree $(l-1)$ in $k$ variables. Thus, it is equal to $\left(\begin{array}{c}k+l-2 \\ k-1\end{array}\right) \leqslant(k+l-2)$ !. 
The double divided differences $[\kappa]_{(k, l)}$ will appear in the coefficients of the variance matrix of the Gaussian vector $[\mathrm{ev}]_{x}(f)$. The key step in this direction is the following lemma. It also shows how to compute efficiently $[\kappa]_{(k, l)}$ from the values of $\kappa$ and its derivatives. Finally, Lemma 5.22 shows that taking divided differences in the $x$ variable then in the $y$ variable gives the same result as the converse, which is hinted by the notation but is not obvious from the definition.

Lemma 5.22. Let $p \in \mathbb{N}^{*}$ and let $k, l \in \mathbb{N}^{*}$ be such that $k+l \leqslant p+1$. Let $\kappa \in \mathcal{C}^{p-1}(\mathbb{R})$, for all $x=\left(x_{i}\right)_{1 \leqslant i \leqslant k} \in \mathbb{R}^{k}$ and $y=\left(y_{j}\right)_{1 \leqslant j \leqslant l} \in \mathbb{R}^{l}$ we have:

$$
\begin{aligned}
&\left([\kappa]_{(i, j)}\left(x_{1}, \ldots, x_{i}, y_{1}, \ldots, y_{j}\right)\right)_{\substack{1 \leqslant i \leqslant k \\
1 \leqslant j \leqslant l}}= \\
& M(x)^{-1}\left(\frac{(-1)^{c_{i}(x)} \kappa^{\left(c_{i}(x)+c_{j}(y)\right)}\left(y_{j}-x_{i}\right)}{c_{i}(x) ! c_{j}(y) !}\right)_{\substack{1 \leqslant i \leqslant k \\
1 \leqslant j \leqslant l}}{ }^{t} M(y)^{-1},
\end{aligned}
$$

where $c_{i}(\cdot)$ is as in Definition 5.1 and $M(\cdot)$ is as in Definitions 5.3. In particular,

$$
\left[[\kappa]_{(k, 1)}(x, \cdot)\right]_{l}(y)=[\kappa]_{(k, l)}(x, y)=\left[[\kappa]_{(1, l)}(\cdot, y)\right]_{k}(x) .
$$

Proof. Let $K:(z, w) \mapsto \kappa(w-z)$. We denote by $C(x, y)$ the matrix

$$
\left(\frac{(-1)^{c_{i}(x)} \kappa^{\left(c_{i}(x)+c_{j}(y)\right)}\left(y_{j}-x_{i}\right)}{c_{i}(x) ! c_{j}(y) !}\right)_{\substack{1 \leqslant i \leqslant k \\ 1 \leqslant j \leqslant l}}=\left(\frac{1}{c_{i}(x) ! c_{j}(y) !} \frac{\partial^{c_{i}(x)+c_{j}(y)} K}{\partial z^{c_{i}(x)} \partial w^{c_{j}(y)}}\left(x_{i}, y_{j}\right)\right)_{\substack{1 \leqslant i \leqslant k \\ 1 \leqslant j \leqslant l}} .
$$

The $j$-th column of $C(x, y)$ equals:

$$
\frac{1}{c_{j}(y) !} \frac{\partial^{c_{j}(y)}}{\partial w^{c_{j}(y)}}{ }_{\mid w=y_{j}}\left(\frac{1}{c_{i}(x) !} \frac{\partial^{c_{i}(x)} K}{\partial z^{c_{i}(x)}}\left(x_{i}, w\right)\right)_{1 \leqslant i \leqslant k}=\frac{1}{c_{j}(y) !} \frac{\partial^{c_{j}(y)}}{\partial w^{c_{j}(y)}}{ }_{\mid w=y_{j}} \operatorname{ev}_{x}(K(\cdot, w)) .
$$

Then, by Lemma 5.9 , the $j$-th column of $M(x)^{-1} C(x, y)$ is:

$$
\begin{aligned}
& \frac{1}{c_{j}(y) !} \frac{\partial^{c_{j}(y)}}{\partial w^{c_{j}(y)}}{ }_{\mid w=y_{j}}[\mathrm{ev}]_{x}(K(\cdot, w))=\left(\frac{1}{c_{j}(y) !}{\frac{\partial^{c_{j}(y)}}{\partial w^{c_{j}(y)}}}_{\mid w=y_{j}}[K(\cdot, w)]_{i}\left(x_{1}, \ldots, x_{i}\right)\right)_{1 \leqslant i \leqslant k} \\
& =\left(\frac{1}{c_{j}(y) !} \frac{\partial^{c_{j}(y)}[\kappa]_{(i, 1)}}{\partial w^{c_{j}(y)}}\left(x_{1}, \ldots, x_{i}, y_{j}\right)\right)_{1 \leqslant i \leqslant k} .
\end{aligned}
$$

This shows that the $i$-th row of $M(x)^{-1} C(x, y)$ equals ${ }^{\mathrm{t}} \mathrm{ev}_{y}\left([\kappa]_{(i, 1)}\left(x_{1}, \ldots, x_{i}, \cdot\right)\right)$. Then, the $i$-th row of $M(x)^{-1} C(x, y)^{\mathrm{t}} M(y)^{-1}$ equals:

$$
\begin{aligned}
{ }^{\mathrm{t}}\left(M(y)^{-1} \operatorname{ev}_{y}\left([\kappa]_{(i, 1)}\left(x_{1}, \ldots, x_{i}, \cdot\right)\right)\right) & ={ }^{\mathrm{t}}[\mathrm{ev}]_{y}\left([\kappa]_{(i, 1)}\left(x_{1}, \ldots, x_{i}, \cdot\right)\right) \\
& =\left(\left[[\kappa]_{(i, 1)}\left(x_{1}, \ldots, x_{i}, \cdot\right)\right]_{j}\left(y_{1}, \ldots, y_{j}\right)\right)_{1 \leqslant j \leqslant l} \\
& =\left([\kappa]_{(i, j)}\left(x_{1}, \ldots, x_{i}, y_{1}, \ldots, y_{j}\right)\right)_{1 \leqslant j \leqslant l} .
\end{aligned}
$$

This proves that the coefficients of $M(x)^{-1} C(x, y)^{\mathrm{t}} M(y)^{-1}$ are as claimed.

By the previous computation the bottom-right coefficient of $M(x)^{-1} C(x, y)^{\mathrm{t}} M(y)^{-1}$ equals $\left[[\kappa]_{(k, 1)}(x, \cdot)\right]_{l}(y)=[\kappa]_{(k, l)}(x, y)$. This reflects the fact that we first multiplied $C(x, y)$ by $M(x)^{-1}$ on the left, thus acting on each column of $C(x, y)$ and taking divided differences in the $x$ variables, then we multiplied the result by ${ }^{\mathrm{t}} M(y)^{-1}$ on the right, thus acting on the rows and taking divided differences in the $y$ variables. If we first multiply $C(x, y)$ by ${ }^{\mathrm{t}} M(y)^{-1}$ on the right then multiply the result by $M(x)^{-1}$ on the left, we first 
act on the rows of $C(x, y)$ then on the columns of $C(x, y)^{\mathrm{t}} M(y)^{-1}$. In this case, we start by computing divided differences in the $y$ variables, then we take divided differences in the $x$ variables. The same kind of computation as above shows that the bottom-right coefficient of $M(x)^{-1} C(x, y)^{\mathrm{t}} M(y)^{-1}$ equals $\left[[\kappa]_{(1, l)}(\cdot, y)\right]_{k}(x)$. Thus, the desired relation is just a consequence of the associativity of the matrix product.

We conclude this section by studying the distribution of the divided differences associated with a regular enough Gaussian process. Note that the following result shows that, if $\kappa$ is the correlation function of a $\mathcal{C}^{p-1}$ Gaussian process, then $[\kappa]_{(k, l)}$ is continuous on $\mathbb{R}^{k} \times \mathbb{R}^{l}$, for all $k, l \in\{1, \ldots, p\}$.

Lemma 5.23 (Distribution of divided differences). Let $p \in \mathbb{N}^{*}$, let $f$ be a stationary centered Gaussian process of class $\mathcal{C}^{p-1}$ and let $\kappa$ denote the correlation function of $f$. The map $x \mapsto[\mathrm{ev}]_{x}(f)$ from $\mathbb{R}^{p}$ to itself defines a continuous centered Gaussian field. Its distribution is characterized by the fact that for all $k, l \in\{1, \ldots, p\}$, for all $x \in \mathbb{R}^{k}$ and $y \in \mathbb{R}^{l}, \mathbb{E}\left[[f]_{k}(x)[f]_{l}(y)\right]=[\kappa]_{(k, l)}(x, y)$. Moreover, the distribution of $\left([\mathrm{ev}]_{x}(f)\right)_{x \in \mathbb{R}^{p}}$ is invariant under the diagonal action of $\mathbb{R}$ on $\mathbb{R}^{p}$ by translation. That is, for all $t \in \mathbb{R}$, we have $\left([\mathrm{ev}]_{x+(t, \ldots, t)}(f)\right)_{x \in \mathbb{R}^{p}}=\left([\mathrm{ev}]_{x}(f)\right)_{x \in \mathbb{R}^{p}}$ in distribution.

Proof. Since $[\mathrm{ev}]_{x}$ is linear for all $x \in \mathbb{R}^{p}$, the finite-dimensional marginal distributions of the field $\left([\mathrm{ev}]_{x}(f)\right)_{x \in \mathbb{R}^{p}}$ are centered and Gaussian. By Lemma 5.17, since $f$ is almost surely $\mathcal{C}^{p-1}$, then $x \mapsto[\mathrm{ev}]_{x}(f)$ is almost surely continuous. Thus, $\left([\mathrm{ev}]_{x}(f)\right)_{x \in \mathbb{R}^{p}}$ is a continuous centered Gaussian field, and characterizing its distribution amounts to computing the variance matrix of $[\mathrm{ev}]_{x}(f)$ and $[\mathrm{ev}]_{y}(f)$ for any $x, y \in \mathbb{R}^{p}$.

Recall that, since $f$ is of class $\mathcal{C}^{p-1}$, its correlation function $\kappa$ is at least $\mathcal{C}^{2 p-2}$. Let $x=\left(x_{i}\right)_{1 \leqslant i \leqslant p}$ and $y=\left(y_{j}\right)_{1 \leqslant j \leqslant p} \in \mathbb{R}^{p}$, by Lemmas 5.9 and 5.22 , the variance matrix of $[\mathrm{ev}]_{x}(f)$ and $[\mathrm{ev}]_{y}(f)$ equals:

$$
\begin{aligned}
\mathbb{E}\left[[\mathrm{ev}]_{x}(f)^{\mathrm{t}}[\mathrm{ev}]_{y}(f)\right] & =M(x)^{-1} \mathbb{E}\left[\mathrm{ev}_{x}(f)^{\mathrm{t}} \operatorname{ev}_{y}(f)\right]^{\mathrm{t}} M(y)^{-1} \\
& =M(x)^{-1}\left(\frac{(-1)^{c_{i}(x)} \kappa^{\left(c_{i}(x)+c_{j}(y)\right)}\left(y_{j}-x_{i}\right)}{c_{i}(x) ! c_{j}(y) !}\right)_{1 \leqslant i, j \leqslant p}{ }^{\mathrm{t}} M(y)^{-1} \\
& =\left([\kappa]_{(i, j)}\left(x_{1}, \ldots, x_{i}, y_{1}, \ldots, y_{j}\right)\right)_{1 \leqslant i, j \leqslant p}
\end{aligned}
$$

where $M(x)$ (resp. $M(y)$ ) is defined in Definitions 5.3, and is invertible by Lemma 5.4. Equivalently, we have $\mathbb{E}\left[[f]_{k}(x)[f]_{l}(y)\right]=[\kappa]_{(k, l)}(x, y)$ for any $x \in \mathbb{R}^{k}$ and $y \in \mathbb{R}^{l}$ with $1 \leqslant k, l \leqslant p$.

By Lemma 5.4, for all $x \in \mathbb{R}^{p}$ and all $t \in \mathbb{R}$, we have $M(x+(t, \ldots, t))=M(x)$. Hence, using Lemma 5.9,

$$
[\mathrm{ev}]_{x+(t, \ldots, t)}(f)=M(x)^{-1} \mathrm{ev}_{x+(t, \ldots, t)}(f) .
$$

The stationarity of $f$ implies that $\left(\mathrm{ev}_{x+(t, \ldots, t)}(f)\right)_{x \in \mathbb{R}^{p}}=\left(\mathrm{ev}_{x}(f)\right)_{x \in \mathbb{R}^{p}}$ in distribution. Thus, $\left([\mathrm{ev}]_{x+(t, \ldots, t)}(f)\right)_{x \in \mathbb{R}^{p}}$ is distributed as $\left(M(x)^{-1} \mathrm{ev}_{x}(f)\right)_{x \in \mathbb{R}^{k}}=\left([\mathrm{ev}]_{x}(f)\right)_{x \in \mathbb{R}^{k}}$. One can also check this distributional invariance directly on the expression of the variance matrix $\mathbb{E}\left[[\mathrm{ev}]_{x}(f)^{\mathrm{t}}[\mathrm{ev}]_{y}(f)\right]$ above.

\section{Kac-Rice densities revisited and clustering}

The purpose of this section is to derive alternative expressions for the Kac-Rice density $\rho_{k}$ defined by Equation (3.3). The upshot is to be able to choose the nicest of these expressions depending on the point $x \in \mathbb{R}^{k}$ we are considering. These new expressions use the divided differences introduced in Section 5. In particular, the divided 
differences allow us to replace $\left(f\left(x_{1}\right), \ldots, f\left(x_{k}\right)\right)$ in Equation (3.3) by another Gaussian vector which is never degenerate, even on the diagonal. We also study the properties of $\rho_{k}$ using these new expressions. This allows us to prove Theorems 1.13 and 1.14.

In Section 6.1, we define a nice partition of $\mathbb{R}^{k}$ that we use in the following. In Section 6.2, we derive the alternative expressions of the Kac-Rice densities that we are interested in, using the formalism of divided differences introduced in Section 5. The main result of Section 6.2 is Proposition 6.23. In Section 6.3, we deduce Theorem 1.13 from Proposition 6.23. In Section 6.4, we introduce notations allowing to study the distribution of the random vectors appearing in the definition of $\rho_{k}$. Then, we study the clustering properties of the Kac-Rice densities in Sections 6.5, 6.6 and 6.7. We prove Theorem 1.14 in Section 6.7. Several results of this section will also be useful in the proof of Theorem 1.6 in Section 7.

\subsection{Graph partitions}

In this section, given a finite set $A \neq \emptyset$ and a scale parameter $\eta \geqslant 0$, we define a partition of the Cartesian product $\mathbb{R}^{A}$ into disjoint pieces. These pieces are indexed by the set $\mathcal{P}_{A}$ of partitions of $A$. In order to do this, we first need to define the graph and the partition associated with a point $x \in \mathbb{R}^{A}$ and the scale parameter $\eta$. Along the way, we also endow $\mathcal{P}_{A}$ with a partially ordered set structure.

Definition 6.1. Let $A$ be a non-empty finite set and let $\eta \geqslant 0$. For any $x=\left(x_{a}\right)_{a \in A} \in \mathbb{R}^{A}$, we define a graph $G_{\eta}(x)$ as follows:

- the vertices of $G_{\eta}(x)$ are the elements of $A$;

- two vertices $a$ and $b \in A$ are joined by an edge of $G_{\eta}(x)$ if and only if $a \neq b$ and $\left|x_{a}-x_{b}\right| \leqslant \eta$.

We are not interested in the graph $G_{\eta}(x)$ itself, but rather in the partition of $A$ defined by its connected components. This partition encodes how the components $\left(x_{a}\right)_{a \in A}$ of $x$ are clustered in $\mathbb{R}$, at scale $\eta$. An example of this construction is given on Figure 1 below.

Definition 6.2. Let $A$ be a non-empty finite set and $\eta \geqslant 0$. We define $\mathcal{I}_{\eta}: \mathbb{R}^{A} \rightarrow \mathcal{P}_{A}$ as follows: for all $x=\left(x_{a}\right)_{a \in A} \in \mathbb{R}^{A}, \mathcal{I}_{\eta}(x)$ is the partition of $A$ given by the connected components of $G_{\eta}(x)$. That is $a$ and $b \in A$ belong to the same element of $\mathcal{I}_{\eta}(x)$ if and only if they are in the same connected component of $G_{\eta}(x)$. An element $I \in \mathcal{I}_{\eta}(x)$, or equivalently the set $\left\{x_{i} \mid i \in I\right\}$, is called a cluster of components of $x$ at scale $\eta$.

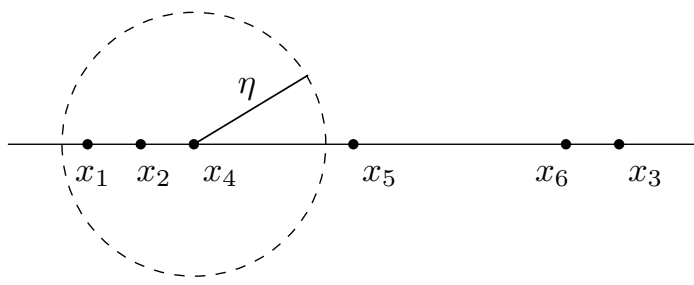

(a) A configuration $x=\left(x_{1}, \ldots, x_{6}\right) \in \mathbb{R}^{6}$. The circle shows the points at distance at most $\eta$ from $x_{4}$.

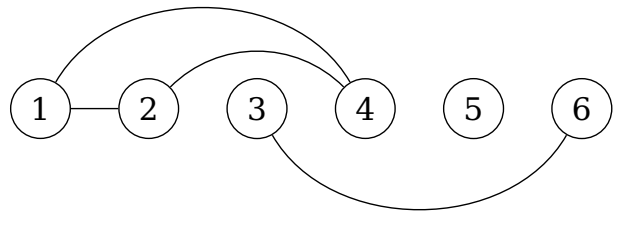

(b) The graph $G_{\eta}(x)$ associated with the configuration $x$ and the distance $\eta$ on the left.

Figure 1: Example of a configuration of six points in $\mathbb{R}$. The partition $\mathcal{I}_{\eta}(x)$ defined by $\eta$ and $x=\left(x_{1}, \ldots, x_{6}\right)$ is $\mathcal{I}_{\eta}(x)=\{\{1,2,4\},\{3,6\},\{5\}\} \in \mathcal{P}_{6}$.

Let us now define a partial order $\leqslant$ on the set $\mathcal{P}_{A}$ of partitions of $A$.

Definition 6.3 (Partial order on partitions). Let $A$ be a non-empty finite set and let $\mathcal{I}, \mathcal{J} \in \mathcal{P}_{A}$. We denote $\mathcal{J} \leqslant \mathcal{I}$ if $\mathcal{J}$ is finer than $\mathcal{I}$, that is for all $J \in \mathcal{J}$, there exists $I \in \mathcal{I}$ 
such that $J \subset I$. We denote by $\mathcal{J}<\mathcal{I}$ the fact that $\mathcal{J} \leqslant \mathcal{I}$ and $\mathcal{I} \neq \mathcal{J}$.

One can check that $\leqslant$ is a partial order on $\mathcal{P}_{A}$ such that $\mathcal{I} \mapsto|\mathcal{I}|$ is decreasing. It admits a minimum equal to $\{\{a\} \mid a \in A\}$, and a maximum equal to $\{A\}$.

Notation 6.4. Let $A \neq \emptyset$ be a finite set, we denote the minimum (resp. maximum) of $\left(\mathcal{P}_{A}, \leqslant\right)$ by $\mathcal{I}_{\min }(A)=\{\{a\} \mid a \in A\}$ (resp. $\left.\mathcal{I}_{\max }(A)=\{A\}\right)$. If $A$ is of the form $\{1, \ldots, k\}$, we use the simpler notation $\mathcal{I}_{\min }(k)$ for $\mathcal{I}_{\min }(A)$ (resp. $\mathcal{I}_{\max }(k)$ for $\mathcal{I}_{\max }(A)$ ).

Let $A$ be a non-empty finite set and let $\mathcal{I}, \mathcal{J} \in \mathcal{P}_{A}$. We have $\mathcal{J} \leqslant \mathcal{I}$ if and only if every $I \in \mathcal{I}$ is obtained as the disjoint union of elements of $\mathcal{J}$. Equivalently, for all $I \in \mathcal{I}$, the set $\{J \in \mathcal{J} \mid J \subset I\}$ is a partition of $I$. This justifies the introduction of the following notation, that will be used in Sections 6.4 and 6.5.

Notation 6.5 (Induced partition). Let $A \neq \emptyset$ be a finite set and let $\mathcal{I}, \mathcal{J} \in \mathcal{P}_{A}$ be such that $\mathcal{J} \leqslant \mathcal{I}$. For all $I \in \mathcal{I}$, we denote by $\mathcal{J}_{I}=\{J \in \mathcal{J} \mid J \subset I\} \in \mathcal{P}_{I}$. Note that if $\mathcal{J} \leqslant \mathcal{I}$ in $\mathcal{P}_{A}$, we have $\mathcal{J}=\bigsqcup_{I \in \mathcal{I}} \mathcal{J}_{I}$.

Lemma 6.6. Let $A$ be a non-empty finite set, for all $x \in \mathbb{R}^{A}$ the map $\eta \mapsto \mathcal{I}_{\eta}(x)$ is non-decreasing from $[0,+\infty)$ to $\mathcal{P}_{A}$.

Proof. Let $x \in \mathbb{R}^{A}$ and let $0 \leqslant \eta \leqslant \eta^{\prime}$. Let $a, b \in A$, if $a$ and $b$ belong to the same cluster of $\mathcal{I}_{\eta}(x)$, then they are in the same connected component of $G_{\eta}(x)$ by definition. Every edge of $G_{\eta}(x)$ is also an edge of $G_{\eta^{\prime}}(x)$, by Definition 6.1. Hence $a$ and $b$ belong to the same cluster of $\mathcal{I}_{\eta^{\prime}}(x)$. Thus $\mathcal{I}_{\eta}(x) \leqslant \mathcal{I}_{\eta^{\prime}}(x)$.

Definition 6.7. Let $A$ be a non-empty finite set, let $\eta \geqslant 0$ and let $\mathcal{I} \in \mathcal{P}_{A}$. We define:

$$
\mathbb{R}_{\mathcal{I}, \eta}^{A}=\left\{x \in \mathbb{R}^{A} \mid \mathcal{I}_{\eta}(x)=\mathcal{I}\right\} .
$$

Remark 6.8. The sets $\mathbb{R}_{\mathcal{I}, \eta}^{A}$ we just defined satisfy the following properties.

- For any $A \neq \emptyset$ and any $\eta \geqslant 0$, we can partition $\mathbb{R}^{A}$ as follows: $\mathbb{R}^{A}=\bigsqcup_{\mathcal{I} \in \mathcal{P}_{A}} \mathbb{R}_{\mathcal{I}, \eta}^{A}$.

- Let $\eta \geqslant 0$, let $\mathcal{I} \in \mathcal{P}_{A}$ and let $\left(x_{a}\right)_{a \in A} \in \mathbb{R}_{\mathcal{I}, \eta}^{A}$. For any cluster $I \in \mathcal{I}$ and any $i, j \in I$, we have: $\left|x_{i}-x_{j}\right| \leqslant(|I|-1) \eta \leqslant|A| \eta$.

Example 6.9. Let $A \neq \emptyset$ be a finite set and let $x=\left(x_{a}\right)_{a \in A} \in \mathbb{R}^{A}$. If $\eta=0$, then $i$ and $j$ are in the same cluster of $\mathcal{I}_{0}(x)$ if and only if $x_{i}=x_{j}$. That is, for all $\mathcal{I} \in \mathcal{P}_{A}$, we have $\mathbb{R}_{\mathcal{I}, 0}^{A}=\Delta_{A, \mathcal{I}}$ (see Definition 2.2). In particular, the partition $\mathcal{I}$ appearing in Theorem 1.13 is $\mathcal{I}_{0}(y)$.

Lemma 6.10. Let $A$ be a non-empty finite set, let $\mathcal{I} \in \mathcal{P}_{A}$ and let $I, J \in \mathcal{I}$ be such that $I \neq J$. Let $\eta \geqslant 0$, for any $x=\left(x_{a}\right)_{a \in A} \in \mathbb{R}_{\mathcal{I}, \eta}^{A}$, we have either $\left(\underline{x}_{J}\right)_{\min }>\left(\underline{x}_{I}\right)_{\max }+\eta$ or $\left(\underline{x}_{J}\right)_{\max }<\left(\underline{x}_{I}\right)_{\min }-\eta$ (see Notations 2.1 and 5.13).

Proof. We can write $I=\left\{i_{1}, \ldots, i_{|I|}\right\}$ in such a way that:

$$
\left(\underline{x}_{I}\right)_{\min }-\eta<x_{i_{1}} \leqslant x_{i_{2}} \leqslant \ldots \leqslant x_{i_{|I|}}<\left(\underline{x}_{I}\right)_{\max }-\eta .
$$

Moreover, since $\mathcal{I}_{\eta}(x)=\mathcal{I}$, we have $x_{i_{k+1}}-x_{i_{k}} \leqslant \eta$ for all $k \in\{1, \ldots,|I|-1\}$. Let $j \in J$, if we had $x_{j} \in\left[\left(\underline{x}_{I}\right)_{\min }-\eta,\left(\underline{x}_{I}\right)_{\max }+\eta\right]$, there would exists $i \in I$ such that $\left|x_{i}-x_{j}\right| \leqslant \eta$. This would contradict the fact that $i$ and $j$ are in different clusters of components of $x$ at scale $\eta$. Hence, for all $j \in J$, we have either $x_{j}>\left(\underline{x}_{I}\right)_{\max }+\eta$ or $x_{j}<\left(\underline{x}_{I}\right)_{\min }-\eta$. Symmetrically, for all $i \in I$, we have either $x_{i}>\left(\underline{x}_{J}\right)_{\max }+\eta$ or $x_{i}<\left(\underline{x}_{J}\right)_{\min }-\eta$.

If we had both $\left(\underline{x}_{J}\right)_{\min }<\left(\underline{x}_{I}\right)_{\min }-\eta$ and $\left(\underline{x}_{J}\right)_{\max }>\left(\underline{x}_{I}\right)_{\max }+\eta$, we would have, for all $i \in I, x_{i} \in\left[\left(\underline{x}_{J}\right)_{\min },\left(\underline{x}_{J}\right)_{\max }\right]$, which is absurd. Hence, either $\left(\underline{x}_{J}\right)_{\min }>\left(\underline{x}_{I}\right)_{\max }+\eta$ or $\left(\underline{x}_{J}\right)_{\max }<\left(\underline{x}_{I}\right)_{\min }-\eta$. 


\subsection{Kac-Rice densities revisited}

The goal of this section is to derive new expressions of the Kac-Rice density $\rho_{k}$ (cf. Definition 3.1), in terms of divided differences studied in Section 5. This is done in Proposition 6.23 below.

In all this section, we denote by $A$ a non-empty finite set and by $f$ a normalized stationary centered Gaussian process which is at least of class $\mathcal{C}^{1}$.

Definition 6.11 (Evaluation map associated with a partition). Let $x=\left(x_{a}\right)_{a \in A} \in \mathbb{R}^{A}$. Using the notations of Section 2.1, for any $\mathcal{I} \in \mathcal{P}_{A}$ we denote by $\operatorname{ev}_{x}^{\mathcal{I}}: \mathcal{C}^{|A|-1}(\mathbb{R}) \rightarrow \mathbb{R}^{|A|}$ the linear map defined by $\mathrm{ev}_{x}^{\mathcal{I}}: f \mapsto\left([\mathrm{ev}]_{\underline{x}_{I}}(f)\right)_{I \in \mathcal{I}}$, where $[\mathrm{ev}]_{x}$ is defined in Definition 5.8.

Remark 6.12. Given $\mathcal{I} \in \mathcal{P}_{A}$, we need to fix an ordering of $\mathcal{I}$ and an ordering of each $I \in \mathcal{I}$ for $\mathrm{ev}_{x}^{\mathcal{I}}$ to be well-defined. Here and throughout the paper, we implicitly assume that such orderings are fixed whenever necessary. The precise choice of these orderings will be of no consequence.

Example 6.13. Let us give some examples.

1. If $\mathcal{I}=\{\{1,3\},\{2,4,5\}\} \in \mathcal{P}_{5}$ and $x=\left(x_{i}\right)_{1 \leqslant i \leqslant 5}$, for all $f \in \mathcal{C}^{4}(\mathbb{R})$ we have:

$$
\operatorname{ev}_{x}^{\mathcal{I}}(f)=\left([f]_{1}\left(x_{1}\right),[f]_{2}\left(x_{1}, x_{3}\right),[f]_{1}\left(x_{2}\right),[f]_{2}\left(x_{2}, x_{4}\right),[f]_{3}\left(x_{2}, x_{4}, x_{5}\right)\right) .
$$

2. For all $x=\left(x_{a}\right)_{a \in A} \in \mathbb{R}^{A}$, we have $\operatorname{ev}_{x}^{\mathcal{I}_{\min }(A)}: f \mapsto\left(f\left(x_{a}\right)\right)_{a \in A}$, where $\mathcal{I}_{\min }(A)$ is as in Notation 6.4.

3. If $\mathcal{I}=\mathcal{I}_{\max }(A)=\{A\}$, then for all $x \in \mathbb{R}^{A}$, we have $\mathrm{ev}_{x}^{\{A\}}=[\mathrm{ev}]_{x}$ after choosing some ordering of $A$. In particular, we have $M(x) \operatorname{ev}_{x}^{\{A\}}=\operatorname{ev}_{x}$ by Lemma 5.7.

4. More generally, let $\mathcal{I} \in \mathcal{P}_{A}$. Let us choose an ordering of each $I \in \mathcal{I}$, and let us also choose an ordering of $\mathcal{I}$, say $\mathcal{I}=\left\{I_{i}|1 \leqslant i \leqslant| I \mid\right\}$. This yields an ordering of $A$ such that if $a \in I_{i}$ and $b \in I_{j}$ with $i<j$ then $a<b$, and whose restriction to any $I \in \mathcal{I}$ coincides with the ordering of $I$ we fixed. Using this ordering, we can identify $A$ with $\{1, \ldots,|A|\}$. Then, for all $x \in \mathbb{R}^{A} \backslash \Delta_{A}$, we have:

$$
\left(\begin{array}{ccc}
M\left(\underline{x}_{I_{1}}\right) & & \\
& \ddots & \\
& & M\left(\underline{x}_{I_{|\mathcal{I}|}}\right)
\end{array}\right) \operatorname{ev}_{x}^{\mathcal{I}}=\mathrm{ev}_{x} .
$$

Note that Equation (6.1) holds independently of our choices or orderings of $\mathcal{I}$ and each $I \in \mathcal{I}$, as long as they are consistent from one term to the other.

Recall that the Kac-Rice densities were defined by Definition 3.1. One of the key ideas in this paper is that we can find alternative expressions of $\rho_{k}$ (see Equation (3.3)). These alternative expressions are indexed by $\mathcal{I} \in \mathcal{P}_{k}$ (see Definition 6.14 below). Then, we are to choose the right $\mathcal{I}$, that is the right expression for $\rho_{k}$, depending on the point $x \in \mathbb{R}^{k}$ at which we want to evaluate $\rho_{k}$.

Definition 6.14 (Kac-Rice densities associated with a partition). Let $A$ be a non-empty finite set and let $f$ be a centered Gaussian process of class $\mathcal{C}^{|A|}$. Let $\mathcal{I} \in \mathcal{P}_{A}$, for all $x=\left(x_{a}\right)_{a \in A} \in \mathbb{R}^{A}$, we denote by

$$
D_{\mathcal{I}}(x)=\operatorname{det}\left(\operatorname{Var}\left(\operatorname{ev}_{x}^{\mathcal{I}}(f)\right)\right) .
$$

Moreover, if $\operatorname{ev}_{x}^{\mathcal{I}}(f)$ is non-degenerate, i.e. if $D_{\mathcal{I}}(x) \neq 0$, we denote by:

$$
N_{\mathcal{I}}(x)=\mathbb{E}\left[\prod_{I \in \mathcal{I}} \prod_{i \in I}\left|[f]_{|I|+1}\left(\underline{x}_{I}, x_{i}\right)\right| \mid \operatorname{ev}_{x}^{\mathcal{I}}(f)=0\right],
$$


the conditional expectation of $\prod_{I \in \mathcal{I}} \prod_{i \in I}\left|[f]_{|I|+1}\left(\underline{x}_{I}, x_{i}\right)\right|$ given that $\operatorname{ev}_{x}^{\mathcal{I}}(f)=0$. Finally we denote by:

$$
\rho_{\mathcal{I}}(x)=\left(\prod_{I \in \mathcal{I}} \prod_{\left\{(i, j) \in I^{2} \mid i \neq j\right\}}\left|x_{i}-x_{j}\right|^{\frac{1}{2}}\right) \frac{N_{\mathcal{I}}(x)}{(2 \pi)^{\frac{|A|}{2}} D_{\mathcal{I}}(x)^{\frac{1}{2}}} .
$$

Remark 6.15. This definition requires some comments.

- Both $D_{\mathcal{I}}(x)$ and $N_{\mathcal{I}}(x)$ only depend on the joint distribution of the divided differences

$$
\left\{[f]_{j}\left(x_{a_{1}}, \ldots, x_{a_{j}}\right)|1 \leqslant j \leqslant| A \mid+1 \text { and } a_{1}, \ldots, a_{j} \in A\right\} .
$$

By Lemma 5.23, this distribution is a centered Gaussian. This remains true after conditioning on $\operatorname{ev}_{x}^{\mathcal{I}}(f)=0$, as soon as $\operatorname{ev}_{x}^{\mathcal{I}}(f)$ is non-degenerate. In particular, $D_{\mathcal{I}}(x), N_{\mathcal{I}}(x)$ and $\rho_{\mathcal{I}}$ are well-defined.

- By Lemma 5.23, the joint distribution of the divided differences (6.5) is invariant by diagonal translation. In particular, for any $x \in \mathbb{R}^{p}$ and any $t \in \mathbb{R}$ we have $D_{\mathcal{I}}(x+(t, \ldots, t))=D_{\mathcal{I}}(x), N_{\mathcal{I}}(x+(t, \ldots, t))=N_{\mathcal{I}}(x)$ and $\rho_{\mathcal{I}}(x+(t, \ldots, t))=\rho_{\mathcal{I}}(x)$.

- The definitions of $D_{\mathcal{I}}(x), N_{\mathcal{I}}(x)$ and $\rho_{\mathcal{I}}(x)$ do not depend on the orderings we choose on $\mathcal{I}$ and on each $I \in \mathcal{I}$. This is not obvious, and will be proved in Lemmas 6.17 and 6.19 below.

Lemma 6.16 (Continuity). If $f$ is of class $\mathcal{C}^{|A|}$, then for all $\mathcal{I} \in \mathcal{P}_{A}$, the maps $D_{\mathcal{I}}, N_{\mathcal{I}}$ and $\rho_{\mathcal{I}}$ are continuous on their domains of definition.

Proof. The proof is similar to that of Lemma 3.9. Let $\mathcal{I} \in \mathcal{P}_{A}$, for all $x=\left(x_{a}\right)_{a \in A} \in \mathbb{R}^{A}$, we denote by $X_{\mathcal{I}}(x)=\operatorname{ev}_{x}^{\mathcal{I}}(x)$ and by $Y_{\mathcal{I}}(x)=\left([f]_{|I|+1}\left(\underline{x}_{I}, x_{i}\right)\right)_{I \in \mathcal{I}, i \in I}$. By Lemma 5.23, $\left(X_{\mathcal{I}}(x), Y_{\mathcal{I}}(x)\right)_{x \in \mathbb{R}^{A}}$ is a continuous centered Gaussian field with values in $\mathbb{R}^{2|A|}$. We write the variance matrix of $\left(X_{\mathcal{I}}(x), Y_{\mathcal{I}}(x)\right)$ by square blocks of size $|A|$ as:

$$
\left(\begin{array}{ll}
\Theta_{\mathcal{I}}(x) & { }^{\mathrm{t}} \Xi_{\mathcal{I}}(x) \\
\Xi_{\mathcal{I}}(x) & \Omega_{\mathcal{I}}(x)
\end{array}\right)
$$

where $\Theta_{\mathcal{I}}, \Xi_{\mathcal{I}}$ and $\Omega_{\mathcal{I}}$ are continuous on $\mathbb{R}^{A}$. Then, $D_{\mathcal{I}}=\operatorname{det}\left(\Theta_{\mathcal{I}}\right)$ is continuous on $\mathbb{R}^{A}$.

If $x \in \mathbb{R}^{A}$ is such that $D_{\mathcal{I}}(x) \neq 0$, then $Y_{\mathcal{I}}(x)$ given that $X_{\mathcal{I}}(x)=0$ is a well-defined centered Gaussian variable, whose variance matrix $\Lambda_{\mathcal{I}}(x)=\Omega_{\mathcal{I}}(x)-\Xi_{\mathcal{I}}(x) \Theta_{\mathcal{I}}(x)^{-1}{ }^{\mathrm{t}} \Xi_{\mathcal{I}}(x)$ depends continuously on $x$. Then, $N_{\mathcal{I}}(x)=\Pi_{|A|}\left(\Lambda_{\mathcal{I}}(x)\right)$, where $\Pi_{|A|}$ is defined by Definition C.1. By Corollary C.3, $\Pi_{|A|}$ is continuous. Hence $N_{\mathcal{I}}$ is continuous on $\left\{x \in \mathbb{R}^{A} \mid D_{\mathcal{I}}(x) \neq 0\right\}$, and so is $\rho_{\mathcal{I}}$.

Lemma 6.17. Let us assume that $f$ is $\mathcal{C}^{|A|}$. Let $\mathcal{I} \in \mathcal{P}_{A}$, for all $x=\left(x_{a}\right)_{a \in A} \in \mathbb{R}^{A}$ we have:

$$
D_{|A|}(x)=\left(\prod_{I \in \mathcal{I}} \prod_{\left\{(i, j) \in I^{2} \mid i \neq j\right\}}\left|x_{i}-x_{j}\right|\right) D_{\mathcal{I}}(x),
$$

where $D_{|A|}$ is the symmetric function defined by Equation (3.1). In particular, $D_{\mathcal{I}}(x)$ is independent of the ordering on $\mathcal{I}$ and of the ordering on each $I \in \mathcal{I}$ used to define $\mathrm{ev}_{x}^{\mathcal{I}}$.

Proof. Let $\mathcal{I} \in \mathcal{P}_{A}$. As in Example 6.13.4, we choose an ordering of each $I \in \mathcal{I}$ and an ordering of $\mathcal{I}$, say $\mathcal{I}=\left\{I_{i}|1 \leqslant i \leqslant| I \mid\right\}$. This defines an ordering of $A$, that coincides with the one on each $I \in \mathcal{I}$ and such that if $i<j$ then the elements of $I_{i}$ are smaller than those 
of $I_{j}$. Let us identify $A$ with $\{1, \ldots,|A|\}$ using this ordering. For all $x=\left(x_{a}\right) \in \mathbb{R}^{A} \backslash \Delta_{A}$, we have:

$$
\left(\begin{array}{ccc}
M\left(\underline{x}_{I_{1}}\right) & & \\
& \ddots & \\
& & M\left(\underline{x}_{I_{|\mathcal{I}|}}\right)
\end{array}\right) \operatorname{ev}_{x}^{\mathcal{I}}(f)=\mathrm{ev}_{x}(f)=\left(f\left(x_{1}\right), \ldots, f\left(x_{|A|}\right)\right) .
$$

Taking the determinant of the variance of these random vectors, we obtain:

$$
D_{|A|}(x)=\left(\prod_{j=1}^{|\mathcal{I}|} \operatorname{det}\left(M\left(\underline{x}_{I_{j}}\right)\right)^{2}\right) D_{\mathcal{I}}(x)=\left(\prod_{I \in \mathcal{I}} \operatorname{det}\left(M\left(\underline{x}_{I}\right)\right)^{2}\right) D_{\mathcal{I}}(x) .
$$

Let $I \in \mathcal{I}$ and let us assume that $I=\left\{i_{1}, \ldots, i_{|I|}\right\}$. Since $x \notin \Delta_{A}$, by Lemma 5.4 we have

$$
\operatorname{det}\left(M\left(\underline{x}_{I}\right)\right)^{2}=\prod_{j=1}^{|I|} \prod_{l=1}^{j-1}\left(x_{i_{j}}-x_{i_{l}}\right)^{2}=\prod_{1 \leqslant l<j \leqslant|I|}\left(x_{i_{j}}-x_{i_{l}}\right)^{2}=\prod_{\left\{(i, j) \in I^{2} \mid i \neq j\right\}}\left|x_{i}-x_{j}\right| .
$$

This proves Equation (6.6) for any $x \in \mathbb{R}^{A} \backslash \Delta_{A}$. Since $\mathbb{R}^{A} \backslash \Delta_{A}$ is dense in $\mathbb{R}^{A}$ and both sides of Equation (6.6) are continuous functions (see Lemmas 3.9 and 6.16), this relation holds for all $x \in \mathbb{R}^{A}$.

By Lemma 3.5, the function $D_{|A|}$ is symmetric on $\mathbb{R}^{|A|}$. Hence the left-hand side of Equation (6.6) does not depend on the ordering of $A$ we used to identify $A$ and $\{1, \ldots,|A|\}$. Let $D_{\mathcal{I}}(x)$ be defined for all $x \in \mathbb{R}^{A}$ by Equation (6.2), and let $\widetilde{D}_{\mathcal{I}}(x)$ be defined similarly but with other choices of ordering on $\mathcal{I}$ or on some $I \in \mathcal{I}$. Using Equation (6.6), for all $x \in \mathbb{R}^{A} \backslash \Delta_{A}$, we have:

$$
D_{\mathcal{I}}(x)=\left(\prod_{I \in \mathcal{I}} \prod_{\left\{(i, j) \in I^{2} \mid i \neq j\right\}}\left|x_{i}-x_{j}\right|\right)^{-1} D_{|A|}(x)=\widetilde{D}_{\mathcal{I}}(x) .
$$

Indeed the middle term does not depend on the ordering of $\mathcal{I}$, nor on the orderings of each $I \in \mathcal{I}$. Then $D_{\mathcal{I}}=\widetilde{D}_{\mathcal{I}}$, since these are continuous functions on $\mathbb{R}^{A}$ that coincide on a dense subset.

Corollary 6.18. Let us assume that $f \in \mathcal{C}^{|A|}$. Let $\mathcal{I}, \mathcal{J} \in \mathcal{P}_{A}$ be such that $\mathcal{J} \leqslant \mathcal{I}$, then for all $x=\left(x_{a}\right)_{a \in A}$ we have:

$$
D_{\mathcal{J}}(x)=\left(\prod_{I \in \mathcal{I}} \prod_{\left\{\left(J, J^{\prime}\right) \in \mathcal{J}_{I}^{2} \mid J \neq J^{\prime}\right\}} \prod_{(i, j) \in J \times J^{\prime}}\left|x_{i}-x_{j}\right|\right) D_{\mathcal{I}}(x),
$$

where for all $I \in \mathcal{I}$, we denoted by $\mathcal{J}_{I}=\{J \in \mathcal{J} \mid J \subset I\} \in \mathcal{P}_{\mathcal{I}}$, as in Notation 6.5.

Proof. By Lemma 6.17, for all $x=\left(x_{a}\right)_{a \in A} \in \mathbb{R}^{A}$ we have:

$$
\begin{aligned}
\left(\prod_{I \in \mathcal{I}} \prod_{\left\{(i, j) \in I^{2} \mid i \neq j\right\}}\left|x_{i}-x_{j}\right|\right) D_{\mathcal{I}}(x) & =\left(\prod_{J \in \mathcal{J}} \prod_{\left\{(i, j) \in J^{2} \mid i \neq j\right\}}\left|x_{i}-x_{j}\right|\right) D_{\mathcal{J}}(x) \\
& =\left(\prod_{I \in \mathcal{I}} \prod_{J \in \mathcal{J}_{I}} \prod_{\left\{(i, j) \in J^{2} \mid i \neq j\right\}}\left|x_{i}-x_{j}\right|\right) D_{\mathcal{J}}(x) .
\end{aligned}
$$

Thus, the result is true for all $x \in \mathbb{R}^{A} \backslash \Delta_{A}$. Since this set is dense in $\mathbb{R}^{A}$ and since $D_{\mathcal{I}}$ and $D_{\mathcal{J}}$ are continuous (see Lemma 6.16), this concludes the proof. 
Lemma 6.19. Let us assume that $f$ is $\mathcal{C}^{|A|}$. Let $\mathcal{I} \in \mathcal{P}_{A}$, for all $x=\left(x_{a}\right)_{a \in A} \in \mathbb{R}^{A}$ such that $D_{|A|}(x) \neq 0$, we have:

$$
N_{|A|}(x)=\left(\prod_{I \in \mathcal{I}} \prod_{\left\{(i, j) \in I^{2} \mid i \neq j\right\}}\left|x_{i}-x_{j}\right|\right) N_{\mathcal{I}}(x)
$$

where $N_{|A|}$ is the symmetric function defined by Equation (3.2). Moreover, for all $x \in \mathbb{R}^{A}$ such that $D_{\mathcal{I}}(x) \neq 0, N_{\mathcal{I}}(x)$ is independent of the ordering on $\mathcal{I}$ and of the ordering on each $I \in \mathcal{I}$.

Proof. Let $\mathcal{I} \in \mathcal{P}_{A}$, as in the proof of Lemma 6.17, we assume that $\mathcal{I}=\left\{I_{i}|1 \leqslant i \leqslant| \mathcal{I} \mid\right\}$ is ordered, as well as each $I \in \mathcal{I}$. This defines an ordering of $A$ that we use to identify $A$ and $\{1, \ldots,|A|\}$. By Lemma 3.5, neither the condition $D_{|A|}(x) \neq 0$ nor the left-hand side of Equation (6.8) depend on a choice of ordering of $A$.

Let $x \in \mathbb{R}^{A}$, if $D_{\mathcal{I}}(x)=0$, then $D_{|A|}(x)=0$, by Lemma 6.17. Conversely, if $x$ is such that $D_{\mathcal{I}}(x) \neq 0$, by continuity of $D_{\mathcal{I}}$ (see Lemma 6.17) there exists a neighborhood $U$ of $x$ on which $D_{\mathcal{I}}$ does not vanish. By Lemma 6.17, for all $y \in U \backslash \Delta_{A}$, we have $D_{|A|}(y) \neq 0$. Thus $\left\{x \in \mathbb{R}^{A} \mid D_{|A|}(x) \neq 0\right\}$ is a dense subset of $\left\{x \in \mathbb{R}^{A} \mid D_{\mathcal{I}}(x) \neq 0\right\}$. In particular, both $N_{|A|}$ and $N_{\mathcal{I}}$ are well-defined on $\left\{x \in \mathbb{R}^{A} \mid D_{|A|}(x) \neq 0\right\} \subset \mathbb{R}^{A} \backslash \Delta_{A}$.

Let $x=\left(x_{a}\right)_{a \in A} \in \mathbb{R}^{A}$ be such that $D_{|A|}(x) \neq 0$. Since $D_{|A|}(x) \neq 0$ we have $x \notin \Delta_{A}$. Then, as in the proof of Lemma 6.17, Equation (6.7) holds under the identification of $A$ and $\{1, \ldots,|A|\}$. By Lemma 5.4, for all $j \in\{1, \ldots,|\mathcal{I}|\}$ the matrix $M\left(\underline{x}_{I_{j}}\right)$ is invertible. Hence, it is equivalent to condition on $f\left(x_{1}\right)=\cdots=f\left(x_{|A|}\right)=0$ and on $\operatorname{ev}_{x}^{\mathcal{I}}(f)=0$. Thus,

$$
N_{|A|}(x)=\mathbb{E}\left[\prod_{I \in \mathcal{I}} \prod_{i \in I}\left|f^{\prime}\left(x_{i}\right)\right| \mid \operatorname{ev}_{x}^{\mathcal{I}}(f)=0\right] .
$$

Let $I=\left\{i_{1}, \ldots, i_{|I|}\right\} \in \mathcal{I}$. The condition $\operatorname{ev}_{x}^{\mathcal{I}}(f)=0$ implies that $[\mathrm{ev}]_{\underline{x}_{I}}(f)=0$, that is $[f]_{j}\left(x_{i_{1}}, \ldots, x_{i_{j}}\right)=0$ for all $j \in\{1, \ldots,|I|\}$. Under this condition, by Example 5.10.3, we have:

$$
f^{\prime}\left(x_{i}\right)=[f]_{|I|+1}\left(x_{i_{1}}, \ldots, x_{i_{|I|}}, x_{i}\right) \prod_{\substack{1 \leqslant j \leqslant|I| \\ i_{j} \neq i}}\left(x_{i}-x_{i_{j}}\right)=[f]_{|I|+1}\left(\underline{x}_{I}, x_{i}\right) \prod_{j \in I \backslash\{i\}}\left(x_{i}-x_{j}\right),
$$

for all $i \in I$. Hence,

$$
\prod_{i \in I}\left|f^{\prime}\left(x_{i}\right)\right|=\left(\prod_{i \in I}\left|[f]_{|I|+1}\left(\underline{x}_{I}, x_{i}\right)\right|\right)\left(\prod_{\left\{(i, j) \in I^{2} \mid i \neq j\right\}}\left|x_{i}-x_{j}\right|\right) .
$$

This proves that Equation (6.8) holds for all $x \in \mathbb{R}^{A}$ such that $D_{|A|}(x) \neq 0$.

Let $N_{\mathcal{I}}(x)$ be defined by Equation (6.3) and let $\widetilde{N}_{\mathcal{I}}$ be defined similarly but with other choices of ordering on $\mathcal{I}$ or on some $I \in \mathcal{I}$. Recall that the function $N_{|A|}$ is symmetric by Lemma 3.5. Using Equation (6.8), for all $x \in \mathbb{R}^{A}$ such that $D_{|A|}(x) \neq 0$ we have:

$$
N_{\mathcal{I}}(x)=\left(\prod_{I \in \mathcal{I}} \prod_{\left\{(i, j) \in I^{2} \mid i \neq j\right\}}\left|x_{i}-x_{j}\right|^{-1}\right) N_{|A|}(x)=\tilde{N}_{\mathcal{I}}(x)
$$

since the middle term does not depend on our choices of orderings on $\mathcal{I}$ and on each $I \in \mathcal{I}$. By Lemmas 6.16 and 6.17, both $N_{\mathcal{I}}$ and $\widetilde{N}_{\mathcal{I}}$ are defined and continuous on $\left\{x \in \mathbb{R}^{k} \mid D_{\mathcal{I}}(x) \neq 0\right\}$. They coincide on the dense subset $\left\{x \in \mathbb{R}^{k} \mid D_{|A|}(x) \neq 0\right\}$, hence everywhere. 
Corollary 6.20. Let us assume that $f \in \mathcal{C}^{|A|}$. Let $\mathcal{I}, \mathcal{J} \in \mathcal{P}_{A}$ be such that $\mathcal{J} \leqslant \mathcal{I}$, then for all $x=\left(x_{a}\right)_{a \in A}$ such that $D_{\mathcal{J}}(x) \neq 0$, we have:

$$
N_{\mathcal{J}}(x)=\left(\prod_{I \in \mathcal{I}} \prod_{\left\{\left(J, J^{\prime}\right) \in \mathcal{J}_{I}^{2} \mid J \neq J^{\prime}\right\}} \prod_{(i, j) \in J \times J^{\prime}}\left|x_{i}-x_{j}\right|\right) N_{\mathcal{I}}(x),
$$

where for all $I \in \mathcal{I}, \mathcal{J}_{I}=\{J \in \mathcal{J} \mid J \subset I\} \in \mathcal{P}_{I}$, as in Notation 6.5.

Proof. Let $x=\left(x_{a}\right)_{a \in A} \in \mathbb{R}^{A}$ be such that $D_{\mathcal{J}}(x) \neq 0$. By Corollary 6.18, we have $D_{\mathcal{I}}(x) \neq 0$ and both $N_{\mathcal{J}}(x)$ and $N_{\mathcal{I}}(x)$ are well-defined. Then, by Lemma 6.19,

$$
\begin{aligned}
\left(\prod_{I \in \mathcal{I}} \prod_{\left\{(i, j) \in I^{2} \mid i \neq j\right\}}\left|x_{i}-x_{j}\right|\right) N_{\mathcal{I}}(x) & =\left(\prod_{J \in \mathcal{J}} \prod_{\left\{(i, j) \in J^{2} \mid i \neq j\right\}}\left|x_{i}-x_{j}\right|\right) N_{\mathcal{J}}(x) \\
& =\left(\prod_{I \in \mathcal{I}} \prod_{J \in \mathcal{J}_{I}} \prod_{\left\{(i, j) \in J^{2} \mid i \neq j\right\}}\left|x_{i}-x_{j}\right|\right) N_{\mathcal{J}}(x) .
\end{aligned}
$$

Thus, the result is true for all $x \in \mathbb{R}^{A} \backslash \Delta_{A}$ such that $D_{\mathcal{J}}(x) \neq 0$. Since $N_{\mathcal{I}}$ and $N_{\mathcal{J}}$ are continuous (see Lemma 6.16) and $\left\{x \in \mathbb{R}^{A} \backslash \Delta_{A} \mid D_{\mathcal{J}}(x) \neq 0\right\}$ is dense in $\left\{x \in \mathbb{R}^{A} \mid D_{\mathcal{J}}(x) \neq 0\right\}$, this concludes the proof.

Recall that, in Theorems 1.6 and 1.14, we consider a normalized stationary centered Gaussian process $f$ whose correlation function $\kappa$ tends to 0 at infinity. In particular, it satisfies the hypotheses of Lemma 2.10.

Lemma 6.21 (Positivity). Let us assume that $f$ is of class $\mathcal{C}^{|A|}$ and that its correlation function $\kappa$ is such that $\kappa(x) \underset{x \rightarrow+\infty}{\longrightarrow} 0$. Let $x \in \mathbb{R}^{A}$ and let us denote by $\mathcal{I}=\mathcal{I}_{0}(x)$. Then $D_{\mathcal{I}}(x)>0$ and $N_{\mathcal{I}}(x)>0$.

Proof. Let $x \in \mathbb{R}^{A}$ and let $\mathcal{I}=\mathcal{I}_{0}(x) \in \mathcal{P}_{A}$ be the partition defined by Definition 6.2. Since $x \in \mathbb{R}_{\mathcal{I}, 0}^{A}=\Delta_{A, \mathcal{I}}$, there exists $y=\left(y_{I}\right)_{I \in \mathcal{I}} \in \mathbb{R}^{\mathcal{I}} \backslash \Delta_{\mathcal{I}}$ such that $x=\iota_{\mathcal{I}}(y)$ (see Definition 2.3 and Remark 2.4). Let $I \in \mathcal{I}$, we have $\underline{x}_{I}=\left(y_{I}, \ldots, y_{I}\right) \in \mathbb{R}^{I}$. Then, by Definition 6.11 and Example 5.10.2, we have $[\mathrm{ev}]_{\underline{x}_{I}}(f)=\left(\frac{f^{(i)}\left(y_{I}\right)}{i !}\right)_{0 \leqslant i<|I|}$ and $\operatorname{ev}_{x}^{\mathcal{I}}(f)=\left(\frac{f^{(i)}\left(y_{I}\right)}{i !}\right)_{I \in \mathcal{I}, 0 \leqslant i<|I|}$. Since $\kappa$ tends to 0 at infinity, this Gaussian vector is non-degenerate by Lemma 2.10, that is $D_{\mathcal{I}}(x)>0$.

The previous expression of $\mathrm{ev}_{x}^{\mathcal{I}}(f)$ shows that the condition $\mathrm{ev}_{x}^{\mathcal{I}}(f)=0$ is equivalent to: $\forall I \in \mathcal{I}, \forall i \in\{0, \ldots,|I|-1\}, f^{(i)}\left(y_{I}\right)=0$. For all $i \in I$, by Example 5.10 .2 we have:

$$
[f]_{|I|+1}\left(\underline{x}_{I}, x_{i}\right)=[f]_{|I|+1}\left(y_{I}, \ldots, y_{I}\right)=\frac{f^{(|I|)}\left(y_{I}\right)}{|I| !} .
$$

Hence,

$$
N_{\mathcal{I}}(x)=\left(\prod_{I \in \mathcal{I}}|I| !^{-|I|}\right) \mathbb{E}\left[\prod_{I \in \mathcal{I}}\left|f^{(\mid I) \mid}\left(y_{I}\right)\right|^{|I|} \mid \forall I \in \mathcal{I}, \forall i \in\{0, \ldots,|I|-1\}, f^{(i)}\left(y_{I}\right)=0\right] .
$$

Let us denote by $U=\left(f^{(i)}\left(y_{I}\right)\right)_{I \in \mathcal{I}, 0 \leqslant i<|I|}$ and by $V=\left(f^{(|I|)}\left(y_{I}\right)\right)_{I \in \mathcal{I}}$. Since $\kappa(x) \underset{x \rightarrow+\infty}{\longrightarrow} 0$, by Lemma 2.10 the centered Gaussian vector $(U, V)$ is non-degenerate. Let us denote by $\left(\begin{array}{cc}A & { }^{\mathrm{t}} B \\ B & C\end{array}\right)$ its block variance matrix. The variance matrix of $V$ given that $U=0$ is 
$C-B A^{-1 \mathrm{t}} B$, see [6, Proposition 1.2]. Note that $C-B A^{-1 \mathrm{t}} B$ is the Schur complement of $A$. In particular,

$$
\operatorname{det}\left(\begin{array}{ll}
A & { }^{\mathrm{t}} B \\
B & C
\end{array}\right)=\operatorname{det}(A) \operatorname{det}\left(C-B A^{-1 \mathrm{t}} B\right)>0 .
$$

Thus, the distribution of $V$ given that $U=0$ is a non-degenerate centered Gaussian. Hence it admits a positive density with respect to the Lebesgue measure of $\mathbb{R}^{\mathcal{I}}$. Finally, by Equation (6.9) we have $N_{\mathcal{I}}(x)>0$, which concludes the proof.

Corollary 6.22 (Vanishing locus). Let us assume that $f$ is of class $\mathcal{C}^{|A|}$ and $\kappa(x) \underset{x \rightarrow+\infty}{\longrightarrow} 0$. Let $\mathcal{I} \in \mathcal{P}_{A}$, for all $x \in \mathbb{R}^{A}, D_{\mathcal{I}}(x)=0$ if and only if there exist $i \in I \in \mathcal{I}$ and $j \in J \in \mathcal{I}$ such that $I \neq J$ and $x_{i}=x_{j}$. Moreover, if $D_{\mathcal{I}}(x) \neq 0$ we have $N_{\mathcal{I}}(x)>0$. In particular, we have $D_{\{A\}}(x)>0$ and $N_{\{A\}}(x)>0$ for all $x \in \mathbb{R}^{A}$.

Proof. Let $\mathcal{I} \in \mathcal{P}_{A}$, by Corollary 6.18, for all $x \in \mathbb{R}^{A}$ we have:

$$
D_{\mathcal{I}}(x)=D_{\{A\}}(x) \prod_{\left\{(I, J) \in \mathcal{I}^{2} \mid I \neq J\right\}} \prod_{(i, j) \in I \times J}\left|x_{i}-x_{j}\right| .
$$

Let $x \in \mathbb{R}^{A}$, we already know that $D_{\{A\}}(x) \geqslant 0$. If $D_{\{A\}}(x)=0$, then by Equation (6.10) we would have $D_{\mathcal{I}}(x)=0$ for all $\mathcal{I} \in \mathcal{P}_{A}$. Since we assumed that $\kappa(x) \underset{x \rightarrow+\infty}{\longrightarrow} 0$, this would contradict the result of Lemma 6.21. Hence, for all $x \in \mathbb{R}^{A}$, we have $D_{\{A\}}(x)>0$. Then, Equation (6.10) shows that $D_{\mathcal{I}}(x)=0$ if and only if there exist $i \in I \in \mathcal{I}$ and $j \in J \in \mathcal{I}$ such that $I \neq J$ and $x_{i}=x_{j}$.

Similarly, let $x \in \mathbb{R}^{A}$. Since $D_{\{A\}}(x)>0$, we know that $N_{\{A\}}(x)$ is well-defined and non-negative. For all $\mathcal{I} \in \mathcal{P}_{A}$ such that $D_{\mathcal{I}}(x) \neq 0$, we have:

$$
N_{\mathcal{I}}(x)=N_{\{A\}}(x) \prod_{\left\{(I, J) \in \mathcal{I}^{2} \mid I \neq J\right\}} \prod_{(i, j) \in I \times J}\left|x_{i}-x_{j}\right|,
$$

by Corollary 6.20. If we had $N_{\{A\}}(x)=0$, we would have $N_{\mathcal{I}}(x)=0$ for all $\mathcal{I} \in \mathcal{P}_{A}$ such that $D_{\mathcal{I}}(x)>0$, which would contradict Lemma 6.21. Thus, $N_{\{A\}}(x)>0$ for all $x \in \mathbb{R}^{A}$. Finally, by Equations (6.10) and (6.11), if $\mathcal{I} \in \mathcal{P}_{A}$ is such that $D_{\mathcal{I}}(x) \neq 0$, then $N_{\mathcal{I}}(x)>0$.

We can now state the main result of this section.

Proposition 6.23 (Equality of Kac-Rice densities). Let $f$ be a normalized stationary centered Gaussian process whose correlation function tends to 0 at infinity. Let $A$ be a non-empty finite set and us assume that $f$ is of class $\mathcal{C}^{|A|}$. Then, $\rho_{\{A\}}$ is a well-defined continuous map from $\mathbb{R}^{A}$ to $\mathbb{R}$ such that for all $x \in \mathbb{R}^{A}$, for all $\mathcal{I} \in \mathcal{P}_{A}$, if $D_{\mathcal{I}}(x) \neq 0$ then $\rho_{\{A\}}(x)=\rho_{\mathcal{I}}(x)$. Moreover, $\rho_{\{A\}}(x)=0$ if and only if $x \in \Delta_{A}$.

In particular, if $f$ is of class $\mathcal{C}^{k}$ with $k \in \mathbb{N}^{*}$, then the Kac-Rice density $\rho_{k}$ (see Definition 3.1) can be uniquely extended into a continuous map from $\mathbb{R}^{k}$ to $\mathbb{R}$ whose vanishing locus is $\Delta_{k}$. Moreover, for all $x \in \mathbb{R}^{k}$, for all $\mathcal{I} \in \mathcal{P}_{k}$ such that $D_{\mathcal{I}}(x) \neq 0$, we have $\rho_{k}(x)=\rho_{\mathcal{I}}(x)$.

Proof. Let $A \neq \emptyset$ be a finite set and let us assume that $f$ is $\mathcal{C}^{|A|}$. By Corollary 6.22, the maps $N_{\{A\}}$ and $\rho_{\{A\}}$ are well-defined from $\mathbb{R}^{A}$ to $\mathbb{R}$. By Lemma 6.16 and Equation (6.4), these maps are continuous on $\mathbb{R}^{A}$ and $\rho_{\{A\}}$ vanishes along $\Delta_{A}$. In fact, by Corollary 6.22, we have $\rho_{\{A\}}(x)=0$ if and only if $x \in \Delta_{A}$.

Let $\mathcal{I} \in \mathcal{P}_{A}$, by Corollary 6.22, for all $x \in \mathbb{R}^{A} \backslash \Delta_{A}$ we have $D_{\mathcal{I}}(x) \neq 0$. Then, by Corollaries 6.18 and 6.20, for all $x \in \mathbb{R}^{A} \backslash \Delta_{A}, \rho_{\mathcal{I}}(x)=\rho_{\{A\}}(x)$. Since $\rho_{\mathcal{I}}$ and $\rho_{\{A\}}$ are 
continuous and $\mathbb{R}^{A} \backslash \Delta_{A}$ is dense in $\mathbb{R}^{A}$, we have $\rho_{\mathcal{I}}(x)=\rho_{\{A\}}(x)$ for any $x \in \mathbb{R}^{A}$ such that $\rho_{\mathcal{I}}(x)$ is well-defined.

Let $k \in \mathbb{N}^{*}$ and let us assume that $f$ is of class $\mathcal{C}^{k}$. The map $\rho_{k}$ of Equation (3.3) is equal to the map $\rho_{\mathcal{I}_{\min }(k)}$ of Definition 6.14, where $\mathcal{I}_{\min }(k)=\{\{i\} \mid 1 \leqslant i \leqslant k\}$. Applying the first point of Proposition 6.23 with $A=\{1, \ldots, k\}$ and setting $\mathcal{I}_{\max }(k)=\{\{1, \ldots, k\}\}$, we obtain:

$$
\rho_{k}(x)=\rho_{\mathcal{I}_{\min }(k)}(x)=\rho_{\mathcal{I}_{\max }(k)}(x),
$$

for all $x \in \mathbb{R}^{k} \backslash \Delta_{k}$. Then $\rho_{\mathcal{I}_{\max }(k)}$ is the desired continuous extension of $\rho_{k}$ to $\mathbb{R}^{k}$. It is necessarily unique by density of $\mathbb{R}^{k} \backslash \Delta_{k}$ in $\mathbb{R}^{k}$.

Remark 6.24. Given any ordering of $A$, we can identify $A$ and $\{1, \ldots,|A|\}$. Then, assuming that $\kappa$ tends to 0 at infinity, we have $\rho_{\{A\}}(x)=\rho_{\mathcal{I}_{\min }(A)}(x)=\rho_{|A|}(x)$ for any $x \in \mathbb{R}^{A} \backslash \Delta_{A}$. Then, by Lemma 3.5, the map $\rho_{\{A\}}$ is symmetric on $\mathbb{R}^{A} \backslash \Delta_{A}$, hence on $\mathbb{R}^{A}$ by continuity. Moreover, for all $\mathcal{I} \in \mathcal{P}_{A}$, the function $\rho_{\mathcal{I}}$ does not depend on the choices of ordering of $\mathcal{I}$ and of each $I \in \mathcal{I}$, by Lemmas 6.17 and 6.19.

\subsection{Proof of Theorem 1.13: vanishing order of the $k$-point function}

In this section we prove Theorem 1.13. This result is a consequence of Lemmas 6.16, 6.17 and 6.19 , that were proved in the previous section.

Proof of Theorem 1.13. Let $k \in \mathbb{N}^{*}$ and let $f$ be a normalized stationary centered Gaussian $\mathcal{C}^{k}$-process. Let $Z=f^{-1}(0)$ be the point process of the zeros of $f$. Let $y=\left(y_{i}\right)_{1 \leqslant i \leqslant k} \in \mathbb{R}^{k}$ and let $\mathcal{I}=\mathcal{I}_{0}(y) \in \mathcal{P}_{k}$. Recalling Definition 6.2, this partition is the only one such that, for any $i, j \in\{1, \ldots, k\}$, we have $\left(y_{i}=y_{j}\right) \Longleftrightarrow(\exists I \in \mathcal{I},\{i, j\} \subset I)$. As in Theorem 1.13 and Remark 2.5, we have $y \in \Delta_{\mathcal{I}, k}$ and there exists a unique $\left(y_{I}\right)_{I \in \mathcal{I}} \in \mathbb{R}^{\mathcal{I}} \backslash \Delta_{\mathcal{I}}$ such that $y=\iota_{\mathcal{I}}\left(\left(y_{I}\right)_{I \in \mathcal{I}}\right)$. It is characterized by the fact that $y_{I}$ is the common value of the $\left(y_{i}\right)_{i \in \mathcal{I}}$, for all $I \in \mathcal{I}$.

Let us assume that $\left(f^{(i)}\left(y_{I}\right)\right)_{I \in \mathcal{I}, 0 \leqslant i<|I|}$ is non-degenerate. As already discussed in the proof of Lemma 6.21, this is equivalent to the non-degeneracy of $\operatorname{ev}_{y}^{\mathcal{I}}(f)$, see Definition 5.1 and Example 5.2.2, hence $D_{\mathcal{I}}(y)>0$. By Lemma 6.16, there exists a neighborhood $U$ of $y$ in $\mathbb{R}^{k}$ such that $D_{\mathcal{I}}(x)>0$ for all $x \in U$. By Lemma 6.17, for all $x \in U \backslash \Delta_{k}$ we have $D_{k}(x)>0$, so that the Kac-Rice density $\rho_{k}$ is well-defined on $U \backslash \Delta_{k}$ (cf. Definition 3.1). Then, by Lemma 3.11, the $k$-point function of the point process $Z$ is well-defined and equal to $\rho_{k}$ on $U \backslash \Delta_{k}$.

By Lemmas 6.17 and 6.19, for any $x \in U \backslash \Delta_{k}$, we have:

$$
\rho_{k}(x)=\frac{N_{k}(x)}{(2 \pi)^{\frac{k}{2}} D_{k}(x)^{\frac{1}{2}}}=\left(\prod_{I \in \mathcal{I}} \prod_{\left\{(i, j) \in I^{2} \mid i \neq j\right\}}\left|x_{i}-x_{j}\right|^{\frac{1}{2}}\right) \frac{N_{\mathcal{I}}(x)}{(2 \pi)^{\frac{p}{2}} D_{\mathcal{I}}(x)^{\frac{1}{2}}}
$$

By continuity of $D_{\mathcal{I}}$ and $N_{\mathcal{I}}$ on $U$ (cf. Lemma 6.16), we have

$$
\left(\prod_{I \in \mathcal{I}} \prod_{\left\{(i, j) \in I^{2} \mid i<j\right\}} \frac{1}{\left|x_{i}-x_{j}\right|}\right) \rho_{k}(x) \underset{x \rightarrow y}{\longrightarrow} \frac{N_{\mathcal{I}}(y)}{(2 \pi)^{\frac{p}{2}} D_{\mathcal{I}}(y)^{\frac{1}{2}}},
$$

and it is enough to check that the right-hand side of the previous equation equals $\ell(y)$ (cf. Equation (1.4)) to prove the first part of Theorem 1.13.

Let $I \in \mathcal{I}$, for all $i \in I$, we have $y_{i}=y_{I}$. Hence, $\operatorname{ev}_{y}^{\mathcal{I}}(f)=\left(\frac{f^{(i)}\left(y_{I}\right)}{i !}\right)_{I \in \mathcal{I}, 0 \leqslant i<|I|}$ and

$$
D_{\mathcal{I}}(y)=\operatorname{det} \operatorname{Var}\left(\operatorname{ev}_{y}^{\mathcal{I}}(f)\right)=\left(\prod_{I \in \mathcal{I}} \prod_{i=0}^{|I|-1} \frac{1}{i !}\right)^{2} \operatorname{det} \operatorname{Var}\left(\left(f^{(i)}\left(y_{I}\right)\right)_{I \in \mathcal{I}, 0 \leqslant i<|I|}\right) .
$$


Combining Equations (6.12) and (6.9), we have $(2 \pi)^{-\frac{p}{2}} N_{\mathcal{I}}(y) D_{\mathcal{I}}(y)^{-\frac{1}{2}}=\ell(y)$, as expected.

Let us now assume that $\left(f^{(i)}\left(y_{I}\right)\right)_{I \in \mathcal{I}, 0 \leqslant i \leqslant|I|}$ is non-degenerate. Proceeding as in the proof of Lemma 6.21, this condition ensures that $N_{\mathcal{I}}(y)>0$. Hence, $\ell(y)>0$, which concludes the proof.

\subsection{Variance and covariance matrices}

In this section, we study the distribution of the random vectors appearing in the definitions of the functions $D_{\mathcal{I}}, N_{\mathcal{I}}$ and $\rho_{I}$ (see Definition 6.14). We introduce notations for their variance and covariance matrices that will be used in the proof of Theorem 1.14. Then, we derive some estimates for the coefficients of these matrices. In all this section, we denote by $A$ a non-empty finite set and by $f$ a $\mathcal{C}^{|A|}$ Gaussian process which is assumed to be stationary centered and normalized. Moreover, we denote by $\kappa$ the correlation function of $f$.

Let $\mathcal{I} \in \mathcal{P}_{A}$ and $x \in \mathbb{R}^{A}$, using the same notations as in the proof of Lemma 6.16 above, we denote by $X_{\mathcal{I}}(x)$ and $Y_{\mathcal{I}}(x) \in \mathbb{R}^{|A|}$ the following centered Gaussian vectors:

$$
\begin{aligned}
X_{\mathcal{I}}(x) & =\mathrm{ev}_{x}^{\mathcal{I}}(f), \\
Y_{\mathcal{I}}(x) & =\left([f]_{|I|+1}\left(\underline{x}_{I}, x_{i}\right)\right)_{I \in \mathcal{I}, i \in I},
\end{aligned}
$$

We also denote by:

$$
\left(\begin{array}{ll}
\Theta_{\mathcal{I}}(x) & { }^{\mathrm{t}} \Xi_{\mathcal{I}}(x) \\
\Xi_{\mathcal{I}}(x) & \Omega_{\mathcal{I}}(x)
\end{array}\right)
$$

the variance matrix of $\left(X_{\mathcal{I}}(x), Y_{\mathcal{I}}(x)\right)$, by blocks of size $|A|$. Finally, if $D_{\mathcal{I}}(x) \neq 0$, i.e. if $\Theta_{\mathcal{I}}(x)$ is invertible, we denote by $\Lambda_{\mathcal{I}}(x)$ the variance of $Y_{\mathcal{I}}(x)$ given that $X_{\mathcal{I}}(x)=0$. By [6, Proposition 1.2], we have:

$$
\Lambda_{\mathcal{I}}(x)=\Omega_{\mathcal{I}}(x)-\Xi_{\mathcal{I}}(x) \Theta_{\mathcal{I}}(x)^{-1}{ }^{\mathrm{t}} \Xi_{\mathcal{I}}(x) .
$$

Note that $X_{\mathcal{I}}, Y_{\mathcal{I}}, \Theta_{\mathcal{I}}, \Xi_{I}, \Omega_{\mathcal{I}}$ and $\Lambda_{\mathcal{I}}$ depend on how we order $\mathcal{I}$ and each $I \in \mathcal{I}$, but recall that $D_{\mathcal{I}}, N_{\mathcal{I}}$ and $\rho_{\mathcal{I}}$ do not (cf. Lemmas 6.17 and 6.19).

We have $\Theta_{\mathcal{I}}(x)=\operatorname{Var}\left(X_{\mathcal{I}}(x)\right)=\operatorname{Var}\left(\operatorname{ev}_{x}^{\mathcal{I}}(f)\right)$. By Definition 6.11, we can write $\Theta_{\mathcal{I}}(x)$ as a block matrix indexed by the elements of $\mathcal{I}$ : $\Theta_{\mathcal{I}}(x)=\left(\Theta_{I J}\left(\underline{x}_{I}, \underline{x}_{J}\right)\right)_{I, J \in \mathcal{I}}$, where for any $I, J \in \mathcal{I}$,

$$
\Theta_{I J}\left(\underline{x}_{I}, \underline{x}_{J}\right)=\mathbb{E}\left[\left[\mathrm{ev} \underline{\underline{x}}_{I}(f)^{\mathrm{t}}[\mathrm{ev}]_{\underline{x}_{J}}(f)\right]=\left(\mathbb{E}\left[[f]_{k}\left(x_{i_{1}}, \ldots, x_{i_{k}}\right)[f]_{l}\left(x_{j_{1}}, \ldots, x_{j_{l}}\right)\right]\right)_{\substack{1 \leqslant k \leqslant|I| \\ 1 \leqslant l \leqslant|J|}} .\right.
$$

In this last equation, we denoted by $i_{1}, \ldots, i_{|I|}$ (resp. $j_{1}, \ldots, j_{|J|}$ ) the elements of $I$ (resp. J). By Lemma 5.23, we finally obtain that:

$$
\Theta_{I J}\left(\underline{x}_{I}, \underline{x}_{J}\right)=\left([\kappa]_{(k, l)}\left(x_{i_{1}}, \ldots, x_{i_{k}}, x_{j_{1}}, \ldots, x_{j_{l}}\right)\right)_{\substack{1 \leqslant k \leqslant|I| \\ 1 \leqslant l \leqslant|J|}},
$$

where $[\kappa]_{(k, l)}$ is the double divided differences introduced in Definition 5.20. Similarly, we write $\Xi_{\mathcal{I}}(x)=\left(\Xi_{I J}\left(\underline{x}_{I}, \underline{x}_{J}\right)\right)_{I, J \in \mathcal{I}}, \Omega_{\mathcal{I}}(x)=\left(\Omega_{I J}\left(\underline{x}_{I}, \underline{x}_{J}\right)\right)_{I, J \in \mathcal{I}}$ and $\Lambda_{\mathcal{I}}(x)=\left(\Lambda_{I J}(x)\right)_{I, J \in \mathcal{I}}$ by blocks, where for all $I$ and $J \in \mathcal{I}$ :

$$
\begin{aligned}
& \Xi_{I J}\left(\underline{x}_{I}, \underline{x}_{J}\right)=\left([\kappa]_{(|I|+1, l)}\left(\underline{x}_{I}, x_{i_{k}}, x_{j_{1}}, \ldots, x_{j_{l}}\right)\right)_{\substack{1 \leqslant k \leqslant|I| \\
1 \leqslant l \leqslant|J|}}, \\
& \Omega_{I J}\left(\underline{x}_{I}, \underline{x}_{J}\right)=\left([\kappa]_{(|I|+1,|J|+1)}\left(\underline{x}_{I}, x_{i_{k}}, \underline{x}_{J}, x_{j_{l}}\right)\right)_{\substack{1 \leqslant k \leqslant|I| \\
1 \leqslant l \leqslant|J|}},
\end{aligned}
$$


and, if $D_{\mathcal{I}}(x) \neq 0$,

$$
\Lambda_{I J}(x)=\Omega_{I J}(x)-\left(\Xi_{I K}\left(\underline{x}_{I}, \underline{x}_{K}\right)\right)_{K \in \mathcal{I}} \Theta_{\mathcal{I}}(x)^{-1}\left({ }^{\mathrm{t}} \Xi_{J L}\left(\underline{x}_{J}, \underline{x}_{L}\right)\right)_{L \in \mathcal{I}} .
$$

Note that, unlike $\Theta_{I J}, \Xi_{I J}$ and $\Omega_{I J}$, the function $\Lambda_{I J}$ depends a priori on all the components of $x$. Note also that the diagonal blocks are such that, for any $I \in \mathcal{I}$, $\Theta_{I I}\left(\underline{x}_{I}, \underline{x}_{I}\right)=\Theta_{\{I\}}\left(\underline{x}_{I}\right), \Xi_{I I}\left(\underline{x}_{I}, \underline{x}_{I}\right)=\Xi_{\{I\}}\left(\underline{x}_{I}\right)$ and $\Omega_{I I}\left(\underline{x}_{I}, \underline{x}_{I}\right)=\Omega_{\{I\}}\left(\underline{x}_{I}\right)$.

Notation 6.25 (Sup-norm). Let $U=\left(U_{i j}\right)$ be a matrix, we denote by $\|U\|_{\infty}=\max _{i, j}\left|U_{i j}\right|$ its sup-norm.

Recall that we defined the norms $\|\cdot\|_{k, \eta}$ and $\|\cdot\|_{k}$, for $k \in \mathbb{N}$ and $\eta \geqslant 0$, in Notation 1.3.

Lemma 6.26. For any $\mathcal{I} \in \mathcal{P}_{A}$ and any point $x \in \mathbb{R}^{A}$ we have $\left\|\Theta_{\mathcal{I}}(x)\right\|_{\infty} \leqslant\|\kappa\|_{2|A|}$, $\left\|\Xi_{\mathcal{I}}(x)\right\|_{\infty} \leqslant\|\kappa\|_{2|A|}$ and $\left\|\Omega_{\mathcal{I}}(x)\right\|_{\infty} \leqslant\|\kappa\|_{2|A|}$.

Proof. Let us prove this result for $\Theta_{\mathcal{I}}(x)$. It is enough to prove that for all $I, J \in \mathcal{I}$, we have $\left\|\Theta_{I J}\left(\underline{x}_{I}, \underline{x}_{J}\right)\right\|_{\infty} \leqslant\|\kappa\|_{2|A|}$. Let $I, J \in \mathcal{I}$, let $k \in\{1, \ldots,|I|\}$ and let $l \in\{1, \ldots,|J|\}$. By Lemma 5.21,

$$
\left|[\kappa]_{(k, l)}\left(x_{i_{1}}, \ldots, x_{i_{k}}, x_{j_{1}}, \ldots, x_{j_{l}}\right)\right| \leqslant\|\kappa\|_{k+l-2} \leqslant\|\kappa\|_{2|A|} .
$$

Thus, by Equation (6.17), we have $\left\|\Theta_{I J}\left(\underline{x}_{I}, \underline{x}_{J}\right)\right\|_{\infty} \leqslant\|\kappa\|_{2|A|}$. The proof is similar for $\Xi_{\mathcal{I}}(x)$ and $\Omega_{\mathcal{I}}(x)$, using Equations (6.18) and (6.19).

Lemma 6.27. For any $\mathcal{I} \in \mathcal{P}_{A}$ and any point $x \in \mathbb{R}^{A}$ such that $D_{\mathcal{I}}(x) \neq 0$, we have $\left\|\Lambda_{\mathcal{I}}(x)\right\|_{\infty} \leqslant\|\kappa\|_{2|A|}$.

Proof. Since $\Lambda_{\mathcal{I}}(x)$ is a variance matrix, by the Cauchy-Schwarz Inequality it is enough to prove that its diagonal coefficients are bounded by $\|\kappa\|_{2|A|}$.

Since $\Theta_{\mathcal{I}}(x)$ is a variance matrix of determinant $D_{\mathcal{I}}(x)$, if $D_{\mathcal{I}}(x) \neq 0$ then $\Theta_{\mathcal{I}}(x)$ is symmetric and positive definite. Then $\Theta_{\mathcal{I}}(x)^{-1}$ is also symmetric and positive definite, so that the diagonal coefficients of $\Xi_{\mathcal{I}}(x) \Theta_{\mathcal{I}}(x)^{-1}{ }^{\mathrm{t}} \Xi_{\mathcal{I}}(x)$ are non-negative. Indeed these diagonal coefficients are of the form $Z \Theta_{\mathcal{I}}(x)^{-1 \mathrm{t}} Z$, where $Z$ is one of the rows of $\Xi_{\mathcal{I}}(x)$.

Thus, by Equation (6.16), the diagonal coefficients of $\Lambda_{\mathcal{I}}(x)$ are non-negative and bounded from above by the corresponding diagonal coefficients of $\Omega_{\mathcal{I}}(x)$. Finally, $\left\|\Lambda_{\mathcal{I}}(x)\right\|_{\infty} \leqslant\left\|\Omega_{\mathcal{I}}(x)\right\|_{\infty}$ and the conclusion follows from Lemma 6.26.

Corollary 6.28. There exists $C>0$ such that, for all $\mathcal{I} \in \mathcal{P}_{A}$, for all $x \in \mathbb{R}^{A}$ such that $D_{\mathcal{I}}(x) \neq 0$, we have $\left|N_{\mathcal{I}}(x)\right| \leqslant C$.

Proof. We use the notations of Appendix C, in particular Definition C.1. Let $\mathcal{I} \in \mathcal{P}_{A}$, for all $x \in \mathbb{R}^{A}$ such that $D_{\mathcal{I}}(x) \neq 0$, we have $N_{\mathcal{I}}(x)=\Pi_{|A|}\left(\Lambda_{\mathcal{I}}(x)\right)$. By Lemma 6.27, the map $\Lambda_{\mathcal{I}}$ takes values in the compact ball of center 0 and radius $\|\kappa\|_{2|A|}$, in the space of symmetric matrices endowed with $\|\cdot\|_{\infty}$. By Corollary C.3, the map $\Pi_{|A|}$ is continuous on this ball, hence bounded. Thus $N_{\mathcal{I}}$ is bounded on $\left\{x \in \mathbb{R}^{A} \mid D_{\mathcal{I}}(x) \neq 0\right\}$. The conclusion follows from the finiteness of $\mathcal{P}_{A}$.

Lemma 6.29. Let $\mathcal{I}, \mathcal{J} \in \mathcal{P}_{A}$ be such that $\mathcal{J} \leqslant \mathcal{I}$ and let us denote $\mathcal{I}=\left\{I_{1}, \ldots, I_{|\mathcal{I}|}\right\}$. For all $i \in\{1, \ldots,|\mathcal{I}|\}$, we denote by $\mathcal{J}_{i}=\mathcal{J}_{I_{i}}$ (see Notation 6.5) for simplicity. Then, for all 
$\eta \geqslant 0$, for all $x \in \mathbb{R}_{\mathcal{I}, \eta}^{A}$ we have:

$$
\begin{gathered}
\left\|\Theta_{\mathcal{J}}(x)-\left(\begin{array}{cccc}
\Theta_{\mathcal{J}_{1}}\left(\underline{x}_{I_{1}}\right) & 0 & \cdots & 0 \\
0 & \Theta_{\mathcal{J}_{2}}\left(\underline{x}_{I_{2}}\right) & \ddots & \vdots \\
\vdots & \ddots & \ddots & 0 \\
0 & \ldots & 0 & \Theta_{\mathcal{J}_{|\mathcal{I}|}\left(\underline{x}_{I_{|\mathcal{I}|}}\right)}
\end{array}\right)\right\|_{\infty} \leqslant\|\kappa\|_{|A|, \eta}, \\
\left\|\Xi_{\mathcal{J}}(x)-\left(\begin{array}{cccc}
\Xi_{\mathcal{J}_{1}}\left(\underline{x}_{I_{1}}\right) & 0 & \cdots & 0 \\
0 & \Xi_{\mathcal{J}_{2}}\left(\underline{x}_{I_{2}}\right) & \ddots & \vdots \\
\vdots & \ddots & \ddots & 0 \\
0 & \cdots & 0 & \Xi_{\mathcal{J}_{|\mathcal{I}|}}\left(\underline{x}_{I_{|\mathcal{I}|}}\right)
\end{array}\right)\right\|_{\infty} \leqslant\|\kappa\|_{|A|, \eta},
\end{gathered}
$$

and

$$
\left\|\Omega_{\mathcal{J}}(x)-\left(\begin{array}{cccc}
\Omega_{\mathcal{J}_{1}}\left(\underline{x}_{I_{1}}\right) & 0 & \cdots & 0 \\
0 & \Omega_{\mathcal{J}_{2}}\left(\underline{x}_{I_{2}}\right) & \ddots & \vdots \\
\vdots & \ddots & \ddots & 0 \\
0 & \cdots & 0 & \Omega_{\mathcal{J}_{|\mathcal{I}|}}\left(\underline{x}_{I_{|\mathcal{I}|}}\right)
\end{array}\right)\right\|_{\infty} \leqslant\|\kappa\|_{|A|, \eta} .
$$

The statement of Lemma 6.29 may seem a little obscure, so let us start by commenting upon it. In the following, we only consider $\Theta_{\mathcal{J}}(x)$, but the cases of $\Xi_{\mathcal{J}}(x)$ and $\Omega_{\mathcal{J}}(x)$ are similar.

If $\mathcal{J}=\mathcal{I}$, then $\mathcal{J}_{i}=\left\{I \in \mathcal{I} \mid I \subset I_{i}\right\}=\left\{I_{i}\right\}$ for all $i \in\{1, \ldots,|\mathcal{I}|\}$. In this case, using the block decomposition of $\Theta_{\mathcal{I}}(x)$, we have $\Theta_{\mathcal{I}}(x)=\left(\Theta_{I_{i} I_{j}}\left(\underline{x}_{I_{i}}, \underline{x}_{I_{j}}\right)\right)_{1 \leqslant i, j \leqslant|\mathcal{I}|}$ and, by definition, $\Theta_{\mathcal{J}_{i}}\left(\underline{x}_{I_{i}}\right)=\Theta_{\left\{I_{i}\right\}}\left(\underline{x}_{I_{i}}\right)=\Theta_{I_{i} I_{i}}\left(\underline{x}_{I_{i}}, \underline{x}_{I_{i}}\right)$. Then, Lemma 6.29 simply states that the sup-norms of the off-diagonal blocks $\Theta_{I_{i} I_{j}}\left(\underline{x}_{I_{i}}, \underline{x}_{I_{j}}\right)$ with $i \neq j$ are bounded by $\|\kappa\|_{|A|, \eta}$ on $\mathbb{R}_{\mathcal{I}, \eta}^{A}$. This can be deduced from Lemmas 5.21 and 6.10.

It turns out that this result remains true if we refine $\mathcal{I}$ by considering some $\mathcal{J} \leqslant \mathcal{I}$. In this case, for each $i \in\{1, \ldots,|\mathcal{I}|\}$ we need to replace $\left\{I_{i}\right\}$ by another partition of $I_{i}$, namely $\mathcal{J}_{i}$. Then, $\Theta_{\mathcal{J}}(x)$ can be written as a block matrix whose diagonal blocks are the $\Theta_{\mathcal{J}_{i}}\left(\underline{x}_{I_{i}}\right)$ with $i \in\{1, \ldots,|\mathcal{I}|\}$. The proof of Lemma 6.29 in this general case is again a matter of bounding the sup-norm of the off-diagonal blocks in this decomposition of $\Theta_{\mathcal{J}}(x)$.

Proof of Lemma 6.29. We only give a formal proof of the first inequality. The proofs of the other two inequalities are similar.

Let $\mathcal{J} \leqslant \mathcal{I}$, using the same notations as in Equation (6.17), for all $x \in \mathbb{R}^{A}$ we have $\Theta_{\mathcal{J}}(x)=\left(\Theta_{I J}\left(\underline{x}_{I}, \underline{x}_{J}\right)\right)_{I, J \in \mathcal{J}}$, where we fixed some ordering of $\mathcal{J}$. Note that, in the statement of Lemma 6.29 , we implicitly assume that $\mathcal{J}$ is ordered in such a way that if $I \in \mathcal{J}_{i}$ and $J \in \mathcal{J}_{j}$ with $i<j$, then $I$ comes before $J$.

Let us regroup the blocks of $\Theta_{\mathcal{J}}(x)$ according to $\mathcal{I}$. For any $k$ and $l \in\{1, \ldots,|\mathcal{I}|\}$ we denote by $\Theta_{k l}(x)=\left(\Theta_{I J}\left(\underline{x}_{I}, \underline{x}_{J}\right)\right)_{I \in \mathcal{J}_{k}, J \in \mathcal{J}_{l}}$. Then $\Theta_{\mathcal{J}}(x)=\left(\Theta_{k l}(x)\right)_{1 \leqslant k, l \leqslant|\mathcal{I}|}$. Furthermore, for any $k \in\{1, \ldots,|\mathcal{I}|\}$, we have:

$$
\Theta_{k k}(x)=\left(\Theta_{I J}\left(\underline{x}_{I}, \underline{x}_{J}\right)\right)_{I, J \in \mathcal{J}_{k}}=\Theta_{\mathcal{J}_{k}}\left(\underline{x}_{I_{k}}\right) .
$$

Hence, it is enough to prove that $\left\|\Theta_{k l}(x)\right\|_{\infty} \leqslant\|\kappa\|_{|A|, \eta}$, for any $k$ and $l \in\{1, \ldots,|\mathcal{I}|\}$ such that $k \neq l$ and any $x \in \mathbb{R}_{\mathcal{I}, \eta}^{A}$.

Let $k, l \in\{1, \ldots,|\mathcal{I}|\}$ be distinct, let $\eta \geqslant 0$ and let $x \in \mathbb{R}_{\mathcal{I}, \eta}^{A}$. Let $I=\left\{i_{1}, \ldots, i_{|I|}\right\} \in \mathcal{J}_{k}$ and $J=\left\{j_{1}, \ldots, j_{|J|}\right\} \in \mathcal{J}_{l}$, since $I$ and $J$ are disjoint subsets of $A$ we have $|I|+|J| \leqslant|A|$. 
Then, by Equation (6.17), we have:

$$
\Theta_{I J}\left(\underline{x}_{I}, \underline{x}_{J}\right)=\left([\kappa]_{(p, q)}\left(x_{i_{1}}, \ldots, x_{i_{p}}, x_{j_{1}}, \ldots, x_{j_{q}}\right)\right)_{\substack{1 \leqslant p \leqslant|I| \\ 1 \leqslant q \leqslant|J|}} .
$$

By Lemma 5.21, for all $p \in\{1, \ldots,|I|\}$ and all $q \in\{1, \ldots,|J|\}$, we have:

$$
\left|[\kappa]_{(p, q)}\left(x_{i_{1}}, \ldots, x_{i_{p}}, x_{j_{1}}, \ldots, x_{j_{q}}\right)\right| \leqslant \max _{0 \leqslant m \leqslant|A|} \sup _{\left(\underline{x}_{J}\right)_{\min }-\left(\underline{x}_{I}\right)_{\max } \leqslant \xi \leqslant\left(\underline{x}_{J}\right)_{\max }-\left(\underline{x}_{I}\right)_{\min }}\left|\kappa^{(m)}(\xi)\right|,
$$

where we used the facts that $p+q-2 \leqslant|I|+|J| \leqslant|A|$ and:

$$
\begin{array}{ll}
\left(\underline{x}_{I}\right)_{\min } \leqslant \min \left\{x_{i_{1}}, \ldots, x_{i_{p}}\right\}, & \left(\underline{x}_{I}\right)_{\max } \geqslant \max \left\{x_{i_{1}}, \ldots, x_{i_{p}}\right\}, \\
\left(\underline{x}_{J}\right)_{\min } \leqslant \min \left\{x_{j_{1}}, \ldots, x_{j_{q}}\right\}, & \left(\underline{x}_{J}\right)_{\max } \geqslant \max \left\{x_{j_{1}}, \ldots, x_{j_{q}}\right\} .
\end{array}
$$

Since $I \in \mathcal{J}_{k}$, we have $I \subset I_{k}$ so that $\left(\underline{x}_{I}\right)_{\min } \geqslant\left(\underline{x}_{I_{k}}\right)_{\min }$ and $\left(\underline{x}_{I}\right)_{\max } \leqslant\left(\underline{x}_{I_{k}}\right)_{\max }$. Similarly, we have $\left(\underline{x}_{J}\right)_{\min } \geqslant\left(\underline{x}_{I_{l}}\right)_{\min }$ and $\left(\underline{x}_{J}\right)_{\max } \leqslant\left(\underline{x}_{I_{l}}\right)_{\max }$. Hence,

$$
\left(\underline{x}_{I_{l}}\right)_{\min }-\left(\underline{x}_{I_{k}}\right)_{\max } \leqslant\left(\underline{x}_{J}\right)_{\min }-\left(\underline{x}_{I}\right)_{\max } \leqslant\left(\underline{x}_{J}\right)_{\max }-\left(\underline{x}_{I}\right)_{\min } \leqslant\left(\underline{x}_{I_{l}}\right)_{\max }-\left(\underline{x}_{I_{k}}\right)_{\min } .
$$

Since $x \in \mathbb{R}_{\mathcal{I}, \eta}^{A}$, by Lemma 6.10, either $\left(\underline{x}_{I_{l}}\right)_{\min }-\left(\underline{x}_{I_{k}}\right)_{\max } \geqslant \eta$ or $\left(\underline{x}_{I_{l}}\right)_{\max }-\left(\underline{x}_{I_{k}}\right)_{\min } \leqslant-\eta$. In the first case, we have $\left[\left(\underline{x}_{J}\right)_{\min }-\left(\underline{x}_{I}\right)_{\max },\left(\underline{x}_{J}\right)_{\max }-\left(\underline{x}_{I}\right)_{\min }\right] \subset[\eta,+\infty)$, while in the second we have $\left[\left(\underline{x}_{J}\right)_{\min }-\left(\underline{x}_{I}\right)_{\max },\left(\underline{x}_{J}\right)_{\max }-\left(\underline{x}_{I}\right)_{\min }\right] \subset(-\infty,-\eta]$. In both cases, using the parity of $\kappa$ and its derivatives, we get:

$$
\left|[\kappa]_{(p, q)}\left(x_{i_{1}}, \ldots, x_{i_{p}}, x_{j_{1}}, \ldots, x_{j_{q}}\right)\right| \leqslant\|\kappa\|_{|A|, \eta},
$$

for all $p \in\{1, \ldots,|I|\}$ and all $q \in\{1, \ldots,|J|\}$. Thus, $\left\|\Theta_{I J}\left(\underline{x}_{I}, \underline{x}_{J}\right)\right\|_{\infty} \leqslant\|\kappa\|_{|A|, \eta}$ for all $I \in \mathcal{J}_{k}$ and $J \in \mathcal{J}_{l}$. Finally, $\left\|\Theta_{k j}(x)\right\|_{\infty} \leqslant\|\kappa\|_{|A|, \eta}$, which concludes the proof.

Corollary 6.30. There exists $C>0$ such that, for all $\mathcal{I}, \mathcal{J} \in \mathcal{P}_{A}$ such that $\mathcal{J} \leqslant \mathcal{I}$, for all $\eta \geqslant 0$, for all $x \in \mathbb{R}_{\mathcal{I}, \eta}^{A}$ we have:

$$
\left|D_{\mathcal{J}}(x)-\prod_{I \in \mathcal{I}} D_{\mathcal{J}_{I}}\left(\underline{x}_{I}\right)\right| \leqslant C\|\kappa\|_{|A|, \eta}^{2} .
$$

Proof. Let $\mathcal{I}, \mathcal{J} \in \mathcal{P}_{A}$ be such that $\mathcal{J} \leqslant \mathcal{I}$, and let us denote by $I_{1}, \ldots, I_{|\mathcal{I}|}$ the elements of $\mathcal{I}$. As in Lemma 6.29, let us denote $\mathcal{J}_{i}=\mathcal{J}_{I_{i}}$ for all $i \in\{1, \ldots,|\mathcal{I}|\}$.

Let $\eta \geqslant 0$. By Lemma 6.29 , for all $x \in \mathbb{R}_{\mathcal{I}, \eta}^{A}$ we have:

$$
D_{\mathcal{J}}(x)=\operatorname{det} \Theta_{\mathcal{J}}(x)=\prod_{i=1}^{|\mathcal{I}|} \operatorname{det} \Theta_{\mathcal{J}_{i}}\left(\underline{x}_{I_{i}}\right)+O\left(\|\kappa\|_{|A|, \eta}^{2}\right)=\prod_{I \in \mathcal{I}} D_{\mathcal{J}_{I}}\left(\underline{x}_{I}\right)+O\left(\|\kappa\|_{|A|, \eta}^{2}\right) .
$$

Moreover, by Lemma 6.26, the coefficients of $\Theta_{\mathcal{J}}(x)$ are bounded by $\|\kappa\|_{2|A|}$. Hence, the constant implied in the error term $O\left(\|\kappa\|_{|A|, \eta}^{2}\right)$ depends only on $\kappa$ and $|A|$.

\subsection{Denominator clustering}

The purpose of this section is to study the clustering properties of the denominators $D_{\mathcal{I}}$ of the Kac-Rice densities $\rho_{\mathcal{I}}$ (recall Definition 6.14), where $\mathcal{I}$ is a partition of some finite set $A$. In all this section, we consider a finite set $A \neq \emptyset$ and some $\mathcal{C}^{|A|}$ Gaussian process $f$ whose correlation function is denoted by $\kappa$. Recall that $\kappa$ is of class $\mathcal{C}^{2|A|}$ with bounded derivatives of any order up to $2|A|$, and that its norm $\|\kappa\|_{|A|, \eta}$ is defined by Notation 1.3 for any $\eta \geqslant 0$. We assume that $f$ is stationary centered and normalized, and that $\kappa$ tends to 0 at infinity. 
Lemma 6.31. Let $\eta \geqslant 0$, there exists $\varepsilon_{\{A\}, \eta}>0$ such that $\forall x \in \mathbb{R}_{\{A\}, \eta}^{A}, D_{\{A\}}(x) \geqslant \varepsilon_{\{A\}, \eta}$. Proof. By Lemma 6.17, the definition of $D_{\{A\}}$ does not depend on how we ordered the elements of $A$. That is $D_{\{A\}}$ is a symmetric function on $\mathbb{R}^{A}$. Without loss of generality, let us order the elements of $A$, say $A=\left\{a_{i}|1 \leqslant i \leqslant| A \mid\right\}$.

Let $\eta \geqslant 0$ and let $x=\left(x_{a}\right)_{a \in A}$. As explained in Remark 6.15, we have:

$$
D_{\{A\}}(x)=D_{\{A\}}\left(x_{a_{1}}, \ldots, x_{a_{|A|}}\right)=D_{\{A\}}\left(0, x_{a_{2}}-x_{a_{1}}, \ldots, x_{a_{|A|}}-x_{a_{1}}\right) .
$$

Moreover, by Definition 6.7, for all $i \in\{2, \ldots,|A|\}$ we have $\left|x_{a_{i}}-x_{a_{1}}\right| \leqslant|A| \eta$ (see also Remark 6.8). By Lemma 6.16 and Corollary 6.22, the function $D_{\{A\}}$ is continuous and positive on the compact set $\{0\} \times[-|A| \eta,|A| \eta]^{|A|-1}$. Hence, there exists $\varepsilon_{\{A\}, \eta}>0$ such that $D_{\{A\}}(x) \geqslant \varepsilon_{\{A\}, \eta}$ for all $x \in \mathbb{R}_{\{A\}, \eta}^{A}$.

Lemma 6.32 (Uniform lower bound). Let us assume that $\|\kappa\|_{|A|, \eta} \underset{\eta \rightarrow+\infty}{\longrightarrow} 0$. Then, for all $\eta>0$, there exists $\varepsilon_{\eta}>0$ such that $\forall \mathcal{I} \in \mathcal{P}_{A}, \forall x \in \mathbb{R}_{\mathcal{I}, \eta}^{A}, D_{\mathcal{I}}(x) \geqslant \varepsilon_{\eta}$.

Proof. Let us prove that for all $\mathcal{I} \in \mathcal{P}_{A}$ the following statement is true:

$$
\text { for all } \eta>0 \text {, there exists } \varepsilon_{\mathcal{I}, \eta}>0 \text { such that } \forall x \in \mathbb{R}_{\mathcal{I}, \eta}^{A}, D_{\mathcal{I}}(x) \geqslant \varepsilon_{\mathcal{I}, \eta} \text {. }
$$

Since $\mathcal{P}_{A}$ is finite, the conclusion follows by setting $\varepsilon_{\eta}=\min \left\{\varepsilon_{\mathcal{I}, \eta} \mid \mathcal{I} \in \mathcal{P}_{A}\right\}$ for all $\eta>0$.

Recall that we defined a partial order $\leqslant$ on $\mathcal{P}_{A}$ in Definition 6.3, and that the maximum of $\left(\mathcal{P}_{A}, \leqslant\right)$ is $\mathcal{I}_{\max }(A)=\{A\}$. We will prove that (6.21) holds for any $\mathcal{I} \in \mathcal{P}_{A}$ by a backward induction on $\mathcal{I} \in \mathcal{P}_{A}$. If $\mathcal{I}=\mathcal{I}_{\max }(A)=\{A\}$, then (6.21) holds by Lemma 6.31, so we already took care of the base case.

Let $\mathcal{J} \in \mathcal{P}_{A} \backslash\left\{\mathcal{I}_{\max }(A)\right\}$ and let us assume that (6.21) holds for any $\mathcal{I} \in \mathcal{P}_{A}$ such that $\mathcal{J}<\mathcal{I}$. Let $\eta>0$ and let $\tau \geqslant \eta$. If $x \in \mathbb{R}_{\mathcal{J}, \eta}^{A}$, then by Lemma 6.6 we have $\mathcal{I}_{\tau}(x) \geqslant \mathcal{I}_{\eta}(x)=\mathcal{J}$. Hence,

$$
\mathbb{R}_{\mathcal{J}, \eta}^{A}=\mathbb{R}_{\mathcal{J}, \eta}^{A} \cap\left(\bigsqcup_{\mathcal{I} \in \mathcal{P}_{A}} \mathbb{R}_{\mathcal{I}, \tau}^{A}\right)=\bigsqcup_{\mathcal{I} \in \mathcal{P}_{A}}\left(\mathbb{R}_{\mathcal{J}, \eta}^{A} \cap \mathbb{R}_{\mathcal{I}, \tau}^{A}\right)=\bigsqcup_{\mathcal{I} \geqslant \mathcal{J}}\left(\mathbb{R}_{\mathcal{J}, \eta}^{A} \cap \mathbb{R}_{\mathcal{I}, \tau}^{A}\right) .
$$

Let $x \in \mathbb{R}_{\mathcal{J}, \eta}^{A} \cap \mathbb{R}_{\mathcal{J}, \tau}^{A}$, that is the components of $x$ form clusters that are encoded by $\mathcal{J}$, the points of a given cluster are at distance of order $\eta$ from one another, and two distinct clusters are further apart than $\tau$. Since $x \in \mathbb{R}_{\mathcal{J}, \tau}^{A}$, applying Corollary 6.30 with $\mathcal{I}=\mathcal{J}$, we get:

$$
D_{\mathcal{J}}(x)=\prod_{J \in \mathcal{J}} D_{\{J\}}\left(\underline{x}_{J}\right)+O\left(\|\kappa\|_{|A|, \tau}^{2}\right) .
$$

Let $J \in \mathcal{J}$, applying Lemma 6.31 with $A=J$, there exists $\varepsilon_{\{J\}, \eta}>0$ such that $D_{\{J\}}$ is bounded from below by $\varepsilon_{\{J\}, \eta}$ on $\mathbb{R}_{\{J\}, \eta}^{J}$. Since $x \in \mathbb{R}_{\mathcal{J}, \eta}^{A}$, we have $\underline{x}_{J} \in \mathbb{R}_{\{J\}, \eta}^{J}$ and $D_{\{J\}}\left(\underline{x}_{J}\right) \geqslant \varepsilon_{\{J\}, \eta}$. Now, let us assume $\tau \geqslant \eta$ to be large enough for the error term in Equation (6.23) to be bounded by $\frac{1}{2} \prod_{J \in \mathcal{J}} \varepsilon_{\{J\}, \eta}$. This is possible because $\|\kappa\|_{|A|, \tau}$ tends to 0 as $\tau \rightarrow+\infty$. Then, for all $x \in \mathbb{R}_{\mathcal{J}, \eta}^{A} \cap \mathbb{R}_{\mathcal{J}, \tau}^{A}$, we have $D_{\mathcal{J}}(x) \geqslant \frac{1}{2} \prod_{J \in \mathcal{J}} \varepsilon_{\{J\}, \eta}>0$.

Let $\mathcal{I} \in \mathcal{P}_{A}$ be such that $\mathcal{J}<\mathcal{I}$. By Corollary 6.18, for all $x \in \mathbb{R}^{A}$ we have:

$$
D_{\mathcal{J}}(x)=\left(\prod_{I \in \mathcal{I}} \prod_{\left\{\left(J, J^{\prime}\right) \in \mathcal{J}_{I}^{2} \mid J \neq J^{\prime}\right\}} \prod_{(i, j) \in J \times J^{\prime}}\left|x_{i}-x_{j}\right|\right) D_{\mathcal{I}}(x) .
$$

Let $x \in \mathbb{R}_{\mathcal{J}, \eta}^{A} \cap \mathbb{R}_{\mathcal{I}, \tau}^{A}$, where $\tau \geqslant \eta$ is the one we chose previously. Using the induction hypothesis (6.21) for $\mathcal{I}$, there exits $\varepsilon_{\mathcal{I}, \tau}>0$ such that $D_{\mathcal{I}}$ is bounded from below by $\varepsilon_{\mathcal{I}, \tau}$ on $\mathbb{R}_{\mathcal{I}, \tau}^{A}$. In particular, $D_{\mathcal{I}}(x) \geqslant \varepsilon_{\mathcal{I}, \tau}$. Moreover, let $J$ and $J^{\prime} \in \mathcal{J}$ be such that $J \neq J^{\prime}$, 
then for all $i \in J$ and $j \in J^{\prime}$ we have $\left|x_{i}-x_{j}\right|>\eta$. Hence, $D_{\mathcal{J}}(x) \geqslant \eta^{\alpha(\mathcal{I}, \mathcal{J})} \varepsilon_{\mathcal{I}, \tau}>0$, where

$$
\begin{aligned}
\alpha(\mathcal{I}, \mathcal{J}) & =\sum_{I \in \mathcal{I}} \sum_{\left\{\left(J, J^{\prime}\right) \in \mathcal{J}_{I}^{2} \mid J \neq J^{\prime}\right\}}|J|\left|J^{\prime}\right|=\sum_{I \in \mathcal{I}}\left(\left(\sum_{J \in \mathcal{J}_{I}}|J|\right)^{2}-\sum_{J \in \mathcal{J}_{I}}|J|^{2}\right) \\
& =\sum_{I \in \mathcal{I}}|I|^{2}-\sum_{J \in \mathcal{J}}|J|^{2} \geqslant 0 .
\end{aligned}
$$

We set

$$
\varepsilon_{\mathcal{J}, \eta}=\min \left\{\frac{1}{2} \prod_{J \in \mathcal{J}} \varepsilon_{\{J\}, \eta}\right\} \cup\left\{\eta^{\alpha(\mathcal{I}, \mathcal{J})} \varepsilon_{\mathcal{I}, \tau} \mid \mathcal{I}>\mathcal{J}\right\}>0 .
$$

Then, by Equation (6.22), for all $x \in \mathbb{R}_{\mathcal{J}, \eta}^{A}$ we have $D_{\mathcal{J}}(x) \geqslant \varepsilon_{\mathcal{J}, \eta}$. Thus (6.21) holds for $\mathcal{J}$, which concludes the induction step and the proof.

Lemma 6.33 (Denominator clustering). Let us assume that $\|\kappa\|_{|A|, \eta} \underset{\eta \rightarrow+\infty}{\longrightarrow} 0$. Let $\eta \geqslant 1$, let $\mathcal{I}, \mathcal{J} \in \mathcal{P}_{A}$ be such that $\mathcal{J} \leqslant \mathcal{I}$ and let $x \in \mathbb{R}_{\mathcal{J}, 1}^{A} \cap \mathbb{R}_{\mathcal{I}, \eta}^{A}$, we have:

$$
\prod_{I \in \mathcal{I}} D_{\mathcal{J}_{I}}\left(\underline{x}_{I}\right)=D_{\mathcal{J}}(x)\left(1+O\left(\|\kappa\|_{|A|, \eta}^{2}\right)\right),
$$

where $\mathcal{J}_{I}$ is defined as in Notation 6.5 for all $I \in \mathcal{I}$. Moreover, the constant implied in the error term does not depend on $\eta, \mathcal{I}, \mathcal{J}$ nor $x \in \mathbb{R}_{\mathcal{J}, 1}^{A} \cap \mathbb{R}_{\mathcal{I}, \eta}^{A}$.

Proof. Let $\eta \geqslant 1$, let $\mathcal{I}, \mathcal{J} \in \mathcal{P}_{A}$ be such that $\mathcal{J} \leqslant \mathcal{I}$ and let $x \in \mathbb{R}_{\mathcal{J}, 1}^{A} \cap \mathbb{R}_{\mathcal{I}, \eta}^{A}$. Since $x \in \mathbb{R}_{\mathcal{J}, 1}^{A}$ we have $D_{\mathcal{J}}(x) \geqslant \varepsilon_{1}$, where $\varepsilon_{1}>0$ is given by Lemma 6.32. By Corollary 6.30, since $x \in \mathbb{R}_{\mathcal{I}, \eta}^{A}$, we have:

$$
\left|\frac{\prod_{I \in \mathcal{I}} D_{\mathcal{J}_{I}}\left(\underline{x}_{I}\right)}{D_{\mathcal{J}}(x)}-1\right| \leqslant \frac{C\|\kappa\|_{|A|, \eta}^{2}}{D_{\mathcal{J}}(x)} \leqslant \frac{C\|\kappa\|_{|A|, \eta}^{2}}{\varepsilon_{1}},
$$

where $C>0$ is independent of $\mathcal{I}, \mathcal{J}, \eta$ and $x$. This yields the result.

Remark 6.34. In Lemma 6.33, we deal with $x \in \mathbb{R}_{\mathcal{J}, 1}^{A} \cap \mathbb{R}_{\mathcal{I}, \eta}^{A}$ where $\eta \geqslant 1$ and $\mathcal{J} \leqslant \mathcal{I}$. It means that components of $x$ whose indices lie in the same cluster of $\mathcal{J}$ are at distance less than 1, while components whose indices lie in different clusters of $\mathcal{I}$ are further away than $\eta$.

One could be under the impression that the hypothesis that $\mathcal{J} \leqslant \mathcal{I}$ is restrictive. In fact it is not since, if $x \in \mathbb{R}_{\mathcal{I}, \eta}^{A}$ with $\eta \geqslant 1$, then by Lemma 6.6 we have $\mathcal{I}_{1}(x) \leqslant \mathcal{I}_{\eta}(x)=\mathcal{I}$. In particular, for any $\eta \geqslant 1$ and any $\mathcal{I} \in \mathcal{P}_{A}$ we have:

$$
\mathbb{R}_{\mathcal{I}, \eta}^{A}=\mathbb{R}_{\mathcal{I}, \eta}^{A} \cap \bigsqcup_{\mathcal{J} \in \mathcal{P}_{A}} \mathbb{R}_{\mathcal{J}, 1}^{A}=\bigsqcup_{\mathcal{J} \in \mathcal{P}_{A}}\left(\mathbb{R}_{\mathcal{J}, 1}^{A} \cap \mathbb{R}_{\mathcal{I}, \eta}^{A}\right)=\bigsqcup_{\mathcal{J} \leqslant \mathcal{I}}\left(\mathbb{R}_{\mathcal{J}, 1}^{A} \cap \mathbb{R}_{\mathcal{I}, \eta}^{A}\right)
$$

\subsection{Numerator clustering}

Let us now consider the clustering properties of the numerators $N_{\mathcal{I}}$ of the Kac-Rice densities introduced in Definition 6.14. In this section, we aim to prove a result similar to Lemma 6.33 for these functions. This is achieved in Lemma 6.40 below. Once again, in all this section $A$ is a non-empty finite set, and $f$ is a normalized stationary centered $\mathcal{C}^{|A|}$ Gaussian process, whose correlation function $\kappa$ tends to 0 at infinity.

First, we study the variance matrix of the Gaussian vector appearing in the definition of $N_{\mathcal{I}}$. Given $\mathcal{I} \in \mathcal{P}_{A}$ and $x \in \mathbb{R}^{A}$ such that $D_{\mathcal{I}}(x) \neq 0$, recall that $Y_{\mathcal{I}}(x)$ given that $X_{\mathcal{I}}(x)=0$ is a well-defined centered Gaussian vector in $\mathbb{R}^{|A|}$ of variance matrix $\Lambda_{\mathcal{I}}(x)$ (see Equations (6.13), (6.14) and (6.16)). 
Lemma 6.35. Let us assume that $\|\kappa\|_{|A|, \eta} \underset{\eta \rightarrow+\infty}{\longrightarrow} 0$. Let $\mathcal{I}=\left\{I_{1}, \ldots, I_{|\mathcal{I}|}\right\} \in \mathcal{P}_{A}$ and let $\mathcal{J} \in \mathcal{P}_{A}$ be such that $\mathcal{J} \leqslant \mathcal{I}$. For all $i \in\{1, \ldots,|\mathcal{I}|\}$, we denote by $\mathcal{J}_{i}=\mathcal{J}_{I_{i}}$ (see Notation 6.5) for simplicity. Then, for all $\eta \geqslant 1$, for all $x \in \mathbb{R}_{\mathcal{J}, 1}^{A} \cap \mathbb{R}_{\mathcal{I}, \eta}^{A}$ we have:

$$
\Lambda_{\mathcal{J}}(x)=\left(\begin{array}{cccc}
\Lambda_{\mathcal{J}_{1}}\left(\underline{x}_{I_{1}}\right) & 0 & \cdots & 0 \\
0 & \Lambda_{\mathcal{J}_{2}}\left(\underline{x}_{I_{2}}\right) & \ddots & \vdots \\
\vdots & \ddots & \ddots & 0 \\
0 & \cdots & 0 & \Lambda_{\mathcal{J}_{|\mathcal{I}|}\left(\underline{x}_{I_{|\mathcal{I}|}}\right)}
\end{array}\right)+O\left(\|\kappa\|_{|A|, \eta}\right),
$$

where the error term does not depend on $\eta, \mathcal{I}, \mathcal{J}$ nor $x \in \mathbb{R}_{\mathcal{J}, 1}^{A} \cap \mathbb{R}_{\mathcal{I}, \eta}^{A}$.

Proof. First, let us consider $\Theta_{\mathcal{J}}$ (see Equation (6.15)). By Lemma 6.26, for any $x \in \mathbb{R}^{A}$, the symmetric matrix $\Theta_{\mathcal{J}}(x)$ belongs to the compact ball $\mathcal{B}$ of center 0 and radius $\|\kappa\|_{2|A|}$, for the sup-norm. For all $x \in \mathbb{R}_{\mathcal{J}, 1}^{A}$, we have $\operatorname{det}\left(\Theta_{\mathcal{J}}(x)\right)=D_{\mathcal{J}}(x) \geqslant \varepsilon_{1}$, where $\varepsilon_{1}>0$ is given by Lemma 6.32. Hence, $\Theta_{\mathcal{J}}(x)$ belongs to $\mathcal{B} \cap \operatorname{det}^{-1}\left(\left[\varepsilon_{1},+\infty\right)\right)$, which is a compact set of invertible matrices. By continuity of the inverse on this compact set, there exists $C>0$, depending only on $\|\kappa\|_{2|A|}$ and $\varepsilon_{1}$, such that for all $x \in \mathbb{R}_{\mathcal{J}, 1}^{A},\left\|\Theta_{\mathcal{J}}(x)^{-1}\right\|_{\infty} \leqslant C$.

Let $\eta \geqslant 1$ and let $x \in \mathbb{R}_{\mathcal{J}, 1}^{A} \cap \mathbb{R}_{\mathcal{I}, \eta}^{A}$. Since $\mathcal{J} \leqslant \mathcal{I}$, by Lemma 6.29 , we have:

$$
\begin{array}{rccc}
\left(\begin{array}{cccc}
\Theta_{\mathcal{J}_{1}}\left(\underline{x}_{I_{1}}\right) & 0 & \cdots & 0 \\
0 & \Theta_{\mathcal{J}_{2}}\left(\underline{x}_{I_{2}}\right) & \ddots & \vdots \\
\vdots & \ddots & \ddots & 0 \\
0 & \cdots & 0 & \Theta_{\mathcal{J}_{|\mathcal{I}|}\left(\underline{x}_{I_{|\mathcal{I}|}}\right)}
\end{array}\right) & =\Theta_{\mathcal{J}}(x)+O\left(\|\kappa\|_{|A|, \eta}\right) \\
& =\Theta_{\mathcal{J}}(x)\left(\operatorname{Id}_{|A|}+O\left(\|\kappa\|_{|A|, \eta}\right)\right),
\end{array}
$$

where $\operatorname{Id}_{|A|}$ stands for the identity matrix of size $|A|$. Note that we used the fact that $\Theta_{\mathcal{J}}(x)^{-1}$ is bounded to get the second equality, and that the error terms are independent of $\mathcal{I}, \mathcal{J}, \eta$ and $x$. Using once again the boundedness of $\Theta_{\mathcal{J}}(x)^{-1}$, we obtain after taking the inverse:

$$
\begin{array}{cccc}
\left(\begin{array}{cccc}
\Theta_{\mathcal{J}_{1}}\left(\underline{x}_{I_{1}}\right)^{-1} & 0 & \cdots & 0 \\
0 & \Theta_{\mathcal{J}_{2}}\left(\underline{x}_{I_{2}}\right)^{-1} & \ddots & \vdots \\
\vdots & \ddots & \ddots & 0 \\
0 & \cdots & 0 & \Theta_{\mathcal{J}_{|\mathcal{I}|}\left(\underline{x}_{I_{|\mathcal{I}|}}\right)^{-1}}
\end{array}\right) & =\Theta_{\mathcal{J}(x)^{-1}\left(\operatorname{Id}_{|A|}+O\left(\|\kappa\|_{|A|, \eta}\right)\right)} \\
& =\Theta_{\mathcal{J}}(x)^{-1}+O\left(\|\kappa\|_{|A|, \eta}\right),
\end{array}
$$

where the error terms are uniform in $\eta, \mathcal{I}, \mathcal{J}$ and $x$.

In order to conclude the proof, we start from the definition of $\Lambda_{\mathcal{J}}(x)$ (see Equation (6.16)) and use the previous estimates for $\Theta_{\mathcal{J}}(x)^{-1}$. We also use the estimates of Lemma 6.29 for $\Xi_{\mathcal{J}}(x)$ and $\Omega_{\mathcal{J}}(x)$, as well as the uniform boundedness of $\Xi_{\mathcal{J}}$ (see Lemma 6.26) and $\Theta_{\mathcal{J}}(x)^{-1}$. We obtain:

$$
\Lambda_{\mathcal{J}}(x)=\left(\begin{array}{cccc}
\Lambda_{\mathcal{J}_{1}}\left(\underline{x}_{I_{1}}\right) & 0 & \cdots & 0 \\
0 & \Lambda_{\mathcal{J}_{2}}\left(\underline{x}_{I_{2}}\right) & \ddots & \vdots \\
\vdots & \ddots & \ddots & 0 \\
0 & \cdots & 0 & \Lambda_{\mathcal{J}_{|\mathcal{I}|}\left(\underline{x}_{I_{|\mathcal{I}|}}\right)}
\end{array}\right)+O\left(\|\kappa\|_{|A|, \eta}\right),
$$

where the error term does not depend on $\eta \geqslant 1$, nor on $\mathcal{I}$ and $\mathcal{J} \in \mathcal{P}_{A}$ such that $\mathcal{J} \leqslant \mathcal{I}$, nor on $x \in \mathbb{R}_{\mathcal{J}, 1}^{A} \cap \mathbb{R}_{\mathcal{I}, \eta}^{A}$. 
Recall that $N_{\mathcal{I}}(x)=\Pi_{|A|}\left(\Lambda_{\mathcal{I}}(x)\right)$, where $\Pi_{|A|}$ is the function defined by Definition C.1. The estimate of Lemma 6.35 allows to derive the following additive estimate.

Lemma 6.36. Let us assume that $\|\kappa\|_{|A|, \eta} \underset{\eta \rightarrow+\infty}{\longrightarrow} 0$. Let $\eta \geqslant 1$, let $\mathcal{I}, \mathcal{J} \in \mathcal{P}_{A}$ be such that $\mathcal{J} \leqslant \mathcal{I}$ and let $x \in \mathbb{R}_{\mathcal{J}, 1}^{A} \cap \mathbb{R}_{\mathcal{I}, \eta}^{A}$, we have:

$$
N_{\mathcal{J}}(x)=\prod_{I \in \mathcal{I}} N_{\mathcal{J}_{I}}\left(\underline{x}_{I}\right)+O\left(\left(\|\kappa\|_{|A|, \eta}\right)^{\frac{1}{2}}\right),
$$

where the error term does not depend on $\eta, \mathcal{I}, \mathcal{J}$ nor $x \in \mathbb{R}_{\mathcal{J}, 1}^{A} \cap \mathbb{R}_{\mathcal{I}, \eta}^{A}$.

Proof. Let $\eta \geqslant 1$, let $\mathcal{I}, \mathcal{J} \in \mathcal{P}_{A}$ be such that $\mathcal{J} \leqslant \mathcal{I}$ and let $x \in \mathbb{R}_{\mathcal{J}, 1}^{A} \cap \mathbb{R}_{\mathcal{I}, \eta}^{A}$. Since $x \in \mathbb{R}_{\mathcal{J}, 1}^{A}$, we have $D_{\mathcal{J}}(x) \geqslant \varepsilon_{1}>0$ (see Lemma 6.32), so that $N_{\mathcal{J}}(x)$ is well-defined. Similarly, for any $I \in \mathcal{I}$, we have $\underline{x}_{I} \in \mathbb{R}_{\mathcal{J}_{I}, 1}^{I}$, so that $N_{\mathcal{J}_{I}}\left(\underline{x}_{I}\right)$ is also well-defined.

Let us denote by $I_{1}, \ldots, I_{|\mathcal{I}|}$ the elements of $\mathcal{I}$. For all $i \in\{1, \ldots,|\mathcal{I}|\}$, we set $\mathcal{J}_{i}=\mathcal{J}_{I_{i}}$, and we denote by:

$$
\widetilde{\Lambda}_{\mathcal{J}}(x)=\left(\begin{array}{cccc}
\Lambda_{\mathcal{J}_{1}}\left(\underline{x}_{I_{1}}\right) & 0 & \cdots & 0 \\
0 & \Lambda_{\mathcal{J}_{2}}\left(\underline{x}_{I_{2}}\right) & \ddots & \vdots \\
\vdots & \ddots & \ddots & 0 \\
0 & \cdots & 0 & \Lambda_{\mathcal{J}_{|\mathcal{I}|}}\left(\underline{x}_{I_{|\mathcal{I}|}}\right)
\end{array}\right) .
$$

By Definitions 6.14 and C.1 and the definition of $\Lambda_{\mathcal{J}}(x)$ (see the beginning of Section 6.4), we have $N_{\mathcal{J}}(x)=\Pi_{|A|}\left(\Lambda_{\mathcal{J}}(x)\right)$. Let $\left(Z_{i}\right)_{1 \leqslant i \leqslant|A|} \sim \mathcal{N}\left(0, \widetilde{\Lambda}_{\mathcal{J}}(x)\right)$ in $\mathbb{R}^{|A|}$, we have in the same way:

$$
\Pi_{|A|}\left(\widetilde{\Lambda}_{\mathcal{J}}(x)\right)=\prod_{i=1}^{|\mathcal{I}|} \mathbb{E}\left[\prod_{j=1}^{\left|I_{i}\right|}\left|Z_{\left|I_{1}\right|+\left|I_{2}\right|+\cdots+\left|I_{i-1}\right|+j}\right|\right]=\prod_{i=1}^{|\mathcal{I}|} \Pi_{\left|I_{i}\right|}\left(\Lambda_{\mathcal{J}_{i}}\left(\underline{x}_{I_{i}}\right)\right)=\prod_{I \in \mathcal{I}} N_{\mathcal{J}_{I}}\left(\underline{x}_{I}\right) .
$$

By Lemma 6.35, we have $\left\|\Lambda_{\mathcal{J}}(x)-\widetilde{\Lambda}_{\mathcal{J}}(x)\right\|_{\infty}=O\left(\|\kappa\|_{|A|, \eta}\right)$. Moreover, we have $\left\|\Lambda_{\mathcal{J}}(x)\right\|_{\infty}=O\left(\|\kappa\|_{2|A|}\right)$ by Lemma 6.27, so that both $\Lambda_{\mathcal{J}}(x)$ and $\widetilde{\Lambda}_{\mathcal{J}}(x)$ lie in a ball of center 0 and radius $O\left(\|\kappa\|_{2|A|}\right)$ in the space of symmetric matrices. Note that the constant implied in these estimates is independent of $\eta, \mathcal{I}, \mathcal{J}$ and $x$. By Corollary C.3, the map $\Pi_{|A|}$ is $\frac{1}{2}$-Hölder on compact sets. Hence, there exists $C>0$, depending only on $|A|$ and $\kappa$, such that:

$$
\begin{aligned}
\left|N_{\mathcal{J}}(x)-\prod_{I \in \mathcal{I}} N_{\mathcal{J}_{I}}\left(\underline{x}_{I}\right)\right| & =\mid \Pi_{|A|}\left(\Lambda_{\mathcal{J}}(x)-\Pi_{|A|}\left(\widetilde{\Lambda}_{\mathcal{J}}(x)\right) \mid\right. \\
& \leqslant C\left\|\Lambda_{\mathcal{J}}(x)-\widetilde{\Lambda}_{\mathcal{J}}(x)\right\|_{\infty}^{\frac{1}{2}} \\
& =O\left(\left(\|\kappa\|_{|A|, \eta}\right)^{\frac{1}{2}}\right),
\end{aligned}
$$

where the constant implied in the error term does not depend on $\eta, \mathcal{I}, \mathcal{J}$ nor $x$.

Corollary 6.37. Let us assume that $\|\kappa\|_{|A|, \eta} \underset{\eta \rightarrow+\infty}{\longrightarrow} 0$. Let $\eta \geqslant 1$ and let $\mathcal{I} \in \mathcal{P}_{A}$, for all $x \in \mathbb{R}_{\mathcal{I}, \eta}^{A}$, we have:

$$
N_{\mathcal{I}}(x)=\prod_{I \in \mathcal{I}} N_{\{I\}}\left(\underline{x}_{I}\right)+O\left(\left(\|\kappa\|_{|A|, \eta}\right)^{\frac{1}{2}}\right)
$$

where the constant implied in the error term does not depend on $\eta, \mathcal{I}$ nor $x \in \mathbb{R}_{\mathcal{I}, \eta}^{A}$. 
Proof. Let $\eta \geqslant 1$ and let $\mathcal{I} \in \mathcal{P}_{A}$. Let $x \in \mathbb{R}_{\mathcal{I}, \eta}^{A}$ and let us denote by $\mathcal{J}=\mathcal{I}_{1}(x)$. By Lemma 6.6, we have $\mathcal{J} \leqslant \mathcal{I}$. Applying Lemma 6.36 and Corollary 6.20, we get:

$$
\left(\prod_{I \in \mathcal{I}} \prod_{\left\{\left(J, J^{\prime}\right) \in \mathcal{J}_{I}^{2} \mid J \neq J^{\prime}\right\}} \prod_{(i, j) \in J \times J^{\prime}}\left|x_{i}-x_{j}\right|\right)\left|N_{\mathcal{I}}(x)-\prod_{I \in \mathcal{I}} N_{\{I\}}\left(\underline{x}_{I}\right)\right|=O\left(\left(\|\kappa\|_{|A|, \eta}\right)^{\frac{1}{2}}\right),
$$

where the constant implied in the error term does not depend on $\eta, \mathcal{I}, \mathcal{J}$ nor $x$. Since $x \in \mathbb{R}_{\mathcal{J}, 1}^{A}$, if $i \in J \in \mathcal{J}$ and $j \in J^{\prime} \in \mathcal{J}$ with $J \neq J^{\prime}$ then $\left|x_{i}-x_{j}\right| \geqslant 1$. Hence,

$$
\left(\prod_{I \in \mathcal{I}} \prod_{\left\{\left(J, J^{\prime}\right) \in \mathcal{J}_{I}^{2} \mid J \neq J^{\prime}\right\}} \prod_{(i, j) \in J \times J^{\prime}}\left|x_{i}-x_{j}\right|\right) \geqslant 1 .
$$

This yields the result.

In the remainder of this section, we show that the additive estimate of Corollary 6.37 yields a multiplicative estimate similar to the one derived in Lemma 6.33. The key step is to prove that $N_{\mathcal{I}}(x)$ is bounded from below by a positive constant of the relevant domain.

Lemma 6.38. Let $\eta \geqslant 0$, there exists $\varepsilon_{\{A\}, \eta}^{\prime}>0$ such that $\forall x \in \mathbb{R}_{\{A\}, \eta^{\prime}}^{A}, N_{\{A\}}(x) \geqslant \varepsilon_{\{A\}, \eta^{\prime}}^{\prime}$.

Proof. The proof is similar to that of Lemma 6.31. Note that $N_{\{A\}}$ is a well-defined continuous positive function on $\mathbb{R}^{A}$, by Lemma 6.16 and Corollary 6.22.

Let $\eta \geqslant 0$. Using the stationarity of $f$ (see Remark 6.15), it is enough to prove that there exists $\varepsilon_{\{A\}, \eta}^{\prime}>0$ such that $N_{\{A\}}(x) \geqslant \varepsilon_{\{A\}, \eta}^{\prime}$ for all $x \in\{0\} \times[-|A| \eta,|A| \eta]^{|A|-1}$. This is true, by compactness of this set.

Lemma 6.39 (Uniform lower bound). Let us assume that $\|\kappa\|_{|A|, \eta} \underset{\eta \rightarrow+\infty}{\longrightarrow} 0$. Then, for all $\eta \geqslant 1$, there exists $\varepsilon_{\eta}^{\prime}>0$ such that $\forall \mathcal{I} \in \mathcal{P}_{A}, \forall x \in \mathbb{R}_{\mathcal{I}, \eta^{\prime}}^{A}, N_{\mathcal{I}}(x) \geqslant \varepsilon_{\eta}^{\prime}$.

Proof. The proof is similar to that of Lemma 6.32. We prove by a backward induction on $\left(\mathcal{P}_{A}, \leqslant\right)$ that for any $\mathcal{I} \in \mathcal{P}_{A}$ the following statement is true:

$$
\text { for all } \eta \geqslant 1 \text {, there exists } \varepsilon_{\mathcal{I}, \eta}^{\prime}>0 \text { such that } \forall x \in \mathbb{R}_{\mathcal{I}, \eta}^{A}, N_{\mathcal{I}}(x) \geqslant \varepsilon_{\mathcal{I}, \eta}^{\prime} \text {. }
$$

Then we set $\varepsilon_{\eta}^{\prime}=\min \left\{\varepsilon_{\mathcal{I}, \eta}^{\prime} \mid \mathcal{I} \in \mathcal{P}_{A}\right\}>0$, which is the lower bound we are looking for.

The base case of the induction is for $\mathcal{I}=\mathcal{I}_{\max }(A)=\{A\}$. It is given by Lemma 6.38.

The induction step is similar to the induction step in the proof of Lemma 6.32. In Lemma 6.32, the two key elements are the additive estimate of Equation (6.23) and the relation given by Corollary 6.18. Here, the analogous results are the additive estimate of Corollary 6.37 and the relation given by Corollary 6.20.

Lemma 6.40 (Numerator clustering). Let us assume that $\|\kappa\|_{|A|, \eta} \underset{\eta \rightarrow+\infty}{\longrightarrow} 0$. Let $\eta \geqslant 1$, let $\mathcal{I}, \mathcal{J} \in \mathcal{P}_{A}$ be such that $\mathcal{J} \leqslant \mathcal{I}$ and let $x \in \mathbb{R}_{\mathcal{J}, 1}^{A} \cap \mathbb{R}_{\mathcal{I}, \eta}^{A}$, we have:

$$
\prod_{I \in \mathcal{I}} N_{\mathcal{J}_{I}}\left(\underline{x}_{I}\right)=N_{\mathcal{J}}(x)\left(1+O\left(\left(\|\kappa\|_{|A|, \eta}\right)^{\frac{1}{2}}\right)\right),
$$

where the error term does not depend on $\eta, \mathcal{I}, \mathcal{J}$ nor $x \in \mathbb{R}_{\mathcal{J}, 1}^{A} \cap \mathbb{R}_{\mathcal{I}, \eta}^{A}$.

Proof. Since, $x \in \mathbb{R}_{\mathcal{J}, 1}^{A} \cap \mathbb{R}_{\mathcal{I}, \eta}^{A}$, we have:

$$
\prod_{I \in \mathcal{I}} N_{\mathcal{J}_{I}}\left(\underline{x}_{I}\right)=N_{\mathcal{J}}(x)+O\left(\left(\|\kappa\|_{|A|, \eta}\right)^{\frac{1}{2}}\right)
$$

by Lemma 6.36. The result follows from the fact that $N_{\mathcal{J}}(x) \geqslant \varepsilon_{1}^{\prime}$, where $\varepsilon_{1}^{\prime}>0$ is given by Lemma 6.39 . 


\subsection{Proof of Theorem 1.14: clustering for $k$-point functions}

In this section we prove Theorem 1.14. This result will be deduced from Lemma 6.41 and Proposition 6.43, which will also be useful in the proof of Theorem 1.6 in Section 7.

As usual, in all this section, $A$ denotes a non-empty finite set and $f$ is a normalized centered stationary Gaussian process of class $\mathcal{C}^{|A|}$ whose correlation function is denoted by $\kappa$. We assume in the following that $\|\kappa\|_{|A|, \eta} \underset{\eta \rightarrow+\infty}{\longrightarrow} 0$. In particular, $\kappa$ tends to 0 at infinity, so that the conclusion of Lemma 2.10 holds true. Under these assumptions, the Kac-Rice density $\rho_{\{A\}}$ is well-defined on $\mathbb{R}^{A}$. Moreover, it coincides with $\rho_{|A|}$ on $\mathbb{R}^{A} \backslash \Delta_{A}$ (see Proposition 6.23), which is also the $|A|$-point function of the point process $Z=f^{-1}(0)$ (see Lemma 3.11).

Lemma 6.41 (Boundedness). If $\|\kappa\|_{|A|, \eta} \underset{\eta \rightarrow+\infty}{\longrightarrow} 0$ then there exists $C>0$ such that, for all $x=\left(x_{a}\right)_{a \in A} \in \mathbb{R}^{A}$,

$$
\rho_{\{A\}}(x) \leqslant C\left(\prod_{a \neq b} \min \left(\left|x_{a}-x_{b}\right|, 1\right)\right)^{\frac{1}{2}} .
$$

In particular, $\rho_{\{A\}}$ is bounded on $\mathbb{R}^{A}$.

Proof. Let $x=\left(x_{a}\right)_{a \in A} \in \mathbb{R}^{A}$ and let us denote by $\mathcal{I}=\mathcal{I}_{1}(x)$ for simplicity. We have $x \in \mathbb{R}_{\mathcal{I}, 1}^{A}$, hence $D_{\mathcal{I}}(x) \geqslant \varepsilon_{1}$, where $\varepsilon_{1}>0$ is given by Lemma 6.32. In particular, by Proposition 6.23, we have $\rho_{\{A\}}(x)=\rho_{\mathcal{I}}(x)$. Then, by Equation (6.4),

$$
\rho_{\{A\}}(x)=\left(\prod_{I \in \mathcal{I}} \prod_{\left\{(i, j) \in I^{2} \mid i \neq j\right\}}\left|x_{i}-x_{j}\right|^{\frac{1}{2}}\right) \frac{N_{\mathcal{I}}(x)}{(2 \pi)^{\frac{|A|}{2}} D_{\mathcal{I}}(x)^{\frac{1}{2}}} .
$$

By Corollary 6.28, there exists $C^{\prime}>0$ independent of $\mathcal{I}$ and $x$ such that $N_{\mathcal{I}}(x) \leqslant C^{\prime}$. Hence,

$$
\rho_{\{A\}}(x) \leqslant \frac{C^{\prime}}{(2 \pi)^{\frac{|A|}{2}}\left(\varepsilon_{1}\right)^{\frac{1}{2}}}\left(\prod_{I \in \mathcal{I}} \prod_{\left\{(i, j) \in I^{2} \mid i \neq j\right\}}\left|x_{i}-x_{j}\right|^{\frac{1}{2}}\right) .
$$

Let $a$ and $b \in A$. If $a$ and $b$ belong to the same cluster of $\mathcal{I}$, then $\left|x_{a}-x_{b}\right| \leqslant|A|$ (see Remark 6.8) and $\left|x_{a}-x_{b}\right| \leqslant|A| \min \left(\left|x_{a}-x_{b}\right|, 1\right)$. If $a$ and $b$ belong to different cluster of $\mathcal{I}$, then $\left|x_{a}-x_{b}\right| \geqslant 1$ by definition, so that $\min \left(\left|x_{a}-x_{b}\right|, 1\right)=1$. Thus,

$$
\begin{aligned}
\prod_{I \in \mathcal{I}} \prod_{\left\{(i, j) \in I^{2} \mid i \neq j\right\}}\left|x_{i}-x_{j}\right|^{\frac{1}{2}} & \leqslant|A|^{|A|^{2}}\left(\prod_{I \in \mathcal{I}} \prod_{\left\{(i, j) \in I^{2} \mid i \neq j\right\}} \min \left(\left|x_{i}-x_{j}\right|, 1\right)\right)^{\frac{1}{2}} \\
& \leqslant|A|^{|A|^{2}}\left(\prod_{\left\{(i, j) \in A^{2} \mid i \neq j\right\}} \min \left(\left|x_{i}-x_{j}\right|, 1\right)\right)^{\frac{1}{2}}
\end{aligned}
$$

This proves the result with $C=|A|^{|A|^{2}} C^{\prime}(2 \pi)^{-\frac{|A|}{2}}\left(\varepsilon_{1}\right)^{-\frac{1}{2}}$.

Remark 6.42. Note that this bound is the best we can hope for near the diagonal, because of Theorem 1.13.

Proposition 6.43 (Clustering). Let $A$ be a non-empty finite set. Let $f$ be a normalized centered stationary Gaussian process of class $\mathcal{C}^{|A|}$, whose correlation function $\kappa$ satisfies $\|\kappa\|_{|A|, \eta} \underset{\eta \rightarrow+\infty}{\longrightarrow} 0$. Then, for all $\eta \geqslant 1$, for all $\mathcal{I} \in \mathcal{P}_{A}$, for all $x \in \mathbb{R}_{\mathcal{I}, \eta}^{A}$, we have:

$$
\prod_{I \in \mathcal{I}} \rho_{\{I\}}\left(\underline{x}_{I}\right)=\rho_{\{A\}}(x)\left(1+O\left(\|\kappa\|_{|A|, \eta}\right)^{\frac{1}{2}}\right),
$$


where the constant involved in the error term does not depend on $\eta, \mathcal{I}$ nor $x$.

Proof. First, note that $\rho_{\{A\}}$ (resp. $\rho_{\{I\}}$ ) is well-defined on $\mathbb{R}^{A}$ (resp. $\mathbb{R}^{I}$ ), see Proposition 6.23. Note also that, for any $\mathcal{J} \in \mathcal{P}_{A}$, we have $\rho_{\{A\}}=\rho_{\mathcal{J}}$ whenever $\rho_{\mathcal{J}}$ is well-defined. In this proof, we use the previous fact, and we choose $\mathcal{J} \in \mathcal{P}_{A}$ depending on the point $x \in \mathbb{R}_{\mathcal{I}, \eta}^{A}$ in order to use the nicest expression of $\rho_{\{A\}}$ we can find.

Let $\eta \geqslant 1$, let $\mathcal{I} \in \mathcal{P}_{A}$ and let $x=\left(x_{a}\right)_{a \in A} \in \mathbb{R}_{\mathcal{I}, \eta}^{A}$. Let us denote by $\mathcal{J}=\mathcal{I}_{1}(x)$, so that $\mathcal{J} \leqslant \mathcal{I}$ and $x \in \mathbb{R}_{\mathcal{J}, 1}^{A} \cap \mathbb{R}_{\mathcal{I}, \eta}^{A}$. By Corollary 6.22, since $x \in \mathbb{R}_{\mathcal{J}, 1}^{A}$, we have $D_{\mathcal{J}}(x)>0$ so that $\rho_{\mathcal{J}}(x)$ is well-defined. Similarly, for any $I \in \mathcal{I}$, we have $\underline{x}_{\mathcal{I}} \in \mathbb{R}_{\mathcal{J}_{I}, 1}^{I}$ so that $\rho_{\mathcal{J}_{I}}\left(\underline{x}_{I}\right)$ is well-defined.

By Proposition 6.23 and Equation (6.4), we have:

$$
\begin{aligned}
\prod_{I \in \mathcal{I}} \rho_{\{I\}}\left(\underline{x}_{I}\right) & =\prod_{I \in \mathcal{I}} \rho_{\mathcal{J}_{I}}\left(\underline{x}_{I}\right) \\
& =\prod_{I \in \mathcal{I}}\left(\left(\prod_{J \in \mathcal{J}_{I}} \prod_{\left\{(i, j) \in J^{2} \mid i \neq j\right\}}\left|x_{i}-x_{j}\right|^{\frac{1}{2}}\right) \frac{N_{\mathcal{J}_{I}}\left(\underline{x}_{I}\right)}{(2 \pi)^{\frac{|I|}{2}} D_{\mathcal{J}_{I}}\left(\underline{x}_{I}\right)^{\frac{1}{2}}}\right) \\
& =\left(\prod_{J \in \mathcal{J}} \prod_{\left\{(i, j) \in J^{2} \mid i \neq j\right\}}\left|x_{i}-x_{j}\right|^{\frac{1}{2}}\right) \frac{\prod_{I \in \mathcal{I}} N_{\mathcal{J}_{I}}\left(\underline{x}_{I}\right)}{(2 \pi)^{\frac{|A|}{2}}\left(\prod_{I \in \mathcal{I}} D_{\mathcal{J}_{I}}\left(\underline{x}_{I}\right)\right)^{\frac{1}{2}}} .
\end{aligned}
$$

Since $x \in \mathbb{R}_{\mathcal{J}, 1}^{A} \cap \mathbb{R}_{\mathcal{I}, \eta}^{A}$, by Lemmas 6.33 and 6.40, we obtain:

$$
\prod_{I \in \mathcal{I}} \rho_{\{I\}}\left(\underline{x}_{I}\right)=\left(\prod_{J \in \mathcal{J}} \prod_{\left\{(i, j) \in J^{2} \mid i \neq j\right\}}\left|x_{i}-x_{j}\right|^{\frac{1}{2}}\right) \frac{N_{\mathcal{J}}(x)}{(2 \pi)^{\frac{|A|}{2}} D_{\mathcal{J}}(x)^{\frac{1}{2}}}\left(1+O\left(\left(\|\kappa\|_{|A|, \eta}\right)^{\frac{1}{2}}\right)\right) .
$$

The conclusion follows from $\rho_{\{A\}}(x)=\rho_{\mathcal{J}}(x)$ and the definition of $\rho_{\mathcal{J}}$, see Equation (6.4) and Proposition 6.23.

In Proposition 6.43, we only consider points $x \in \mathbb{R}_{\mathcal{I}, \eta}^{A}$. In fact, an estimate of the same kind remains valid if we replace $\mathbb{R}_{\mathcal{I}, \eta}^{A}$ with $\bigsqcup_{\mathcal{J} \leqslant \mathcal{I}} \mathbb{R}_{\mathcal{J}, \eta}^{A}$. Equivalently, we only need $\mathcal{I}_{\eta}(x) \leqslant \mathcal{I}$ instead of $\mathcal{I}_{\eta}(x)=\mathcal{I}$. That is, we need the components of $x$ whose indices lie in different clusters of $\mathcal{I}$ to be far from one another, but we do not ask anything regarding components whose indices lie in the same cluster of $\mathcal{I}$. The precise statement is the following.

Corollary 6.44. In the setting of Proposition 6.43, for all $\eta \geqslant 1$, for all $\mathcal{I} \in \mathcal{P}_{A}$, for all $x \in \mathbb{R}^{A}$, such that $\mathcal{I}_{\eta}(x) \leqslant \mathcal{I}$, we have:

$$
\prod_{I \in \mathcal{I}} \rho_{\{I\}}\left(\underline{x}_{I}\right)=\rho_{\{A\}}(x)\left(1+O\left(\|\kappa\|_{|A|, \eta}\right)^{\frac{1}{2}}\right),
$$

where the constant involved in the error term does not depend on $\eta, \mathcal{I}$ nor $x$.

Proof. Let $\eta \geqslant 1$, let $\mathcal{I} \in \mathcal{P}_{A}$ and let $x \in \mathbb{R}^{A}$ such that $\mathcal{I}_{\eta}(x) \leqslant \mathcal{I}$. Let us denote by $\mathcal{J}=\mathcal{I}_{\eta}(x)$ for simplicity. Since $x \in \mathbb{R}_{\mathcal{J}, \eta}^{A}$, by Proposition 6.43 we have:

$$
\begin{aligned}
\rho_{\{A\}}(x) & =\left(\prod_{J \in \mathcal{J}} \rho_{\{J\}}\left(\underline{x}_{J}\right)\right)\left(1+O\left(\left(\|\kappa\|_{|A|, \eta}\right)^{\frac{1}{2}}\right)\right) \\
& =\left(\prod_{I \in \mathcal{I}} \prod_{J \in \mathcal{J}_{I}} \rho_{\{J\}}\left(\underline{x}_{J}\right)\right)\left(1+O\left(\left(\|\kappa\|_{|A|, \eta}\right)^{\frac{1}{2}}\right)\right) .
\end{aligned}
$$


Let $I \in \mathcal{I}$, we have $\underline{x}_{I} \in \mathbb{R}_{\mathcal{J}_{I}, \eta}^{I}$. Using Proposition 6.43 once again, we have:

$$
\prod_{J \in \mathcal{J}_{I}} \rho_{\{J\}}\left(\underline{x}_{J}\right)=\rho_{\{I\}}\left(\underline{x}_{I}\right)\left(1+O\left(\left(\|\kappa\|_{|A|, \eta}\right)^{\frac{1}{2}}\right)\right) .
$$

This yields the result. The uniformity of the error term follows from the finiteness of $A$.

We can now prove Theorem 1.14, which is just a special case of Lemma 6.41 and Corollary 6.44.

Proof of Theorem 1.14. Let $k \in \mathbb{N}^{*}$ and let $f$ be a $\mathcal{C}^{k}$ Gaussian process which is normalized centered and stationary. We assume that its correlation function $\kappa$ satisfies $\|\kappa\|_{k, \eta} \underset{\eta \rightarrow+\infty}{\longrightarrow} 0$. By Lemmas 2.10 and 3.11, for all $l \in\{1, \ldots, k\}$, the $l$-point function of the point process $Z=f^{-1}(0)$ is the Kac-Rice density $\rho_{l}$ defined in Definition 3.1. This function is well-defined on $\mathbb{R}^{l} \backslash \Delta_{l}$ and admits a unique continuous extension to $\mathbb{R}^{l}$, which vanishes on $\Delta_{l}$, by Proposition 6.23.

By Proposition 6.23, the continuous extension of $\rho_{k}$ to $\mathbb{R}^{k}$ is the function $\rho_{\mathcal{I}_{\max }(k)}$. Then, by Lemma 6.41, for all $x=\left(x_{i}\right)_{1 \leqslant i \leqslant k} \in \mathbb{R}^{k} \backslash \Delta_{k}$ we have:

$$
\rho_{k}(x)=\rho_{\mathcal{I}_{\max }(k)}(x) \leqslant C \prod_{1 \leqslant i<j \leqslant k} \max \left(\left|x_{i}-x_{j}\right|, 1\right)
$$

for some positive constant $C$.

Let $\eta \geqslant 1$, let $\mathcal{I} \in \mathcal{P}_{k}$ and let $x=\left(x_{i}\right)_{1 \leqslant i \leqslant k} \in \mathbb{R}^{k} \backslash \Delta_{k}$. The condition:

$$
\forall I, J \in \mathcal{I} \text { such that } I \neq J, \forall i \in I, \forall j \in J,\left|x_{i}-x_{j}\right|>\eta,
$$

appearing in Theorem 1.14 is equivalent to $\mathcal{I}_{\eta}(x) \leqslant \mathcal{I}$. Let us assume that $x$ satisfies this condition. Then, by Corollary 6.44 applied with $A=\{1, \ldots, k\}$, we have:

$$
\prod_{I \in \mathcal{I}} \rho_{\{I\}}\left(\underline{x}_{I}\right)=\rho_{\mathcal{I}_{\max }(k)}(x)\left(1+O\left(\|\kappa\|_{k, \eta}\right)^{\frac{1}{2}}\right) .
$$

Finally, we have the equality $\rho_{k}(x)=\rho_{\mathcal{I}_{\max }(k)}(x)$ and similarly, $\rho_{\{I\}}\left(\underline{x}_{I}\right)=\rho_{|I|}\left(\underline{x}_{I}\right)$ for all $I \in \mathcal{I}$ since $\underline{x}_{I} \in \mathbb{R}^{I} \backslash \Delta_{I}$. Hence the result.

\section{Proof of Theorem 1.6: central moments asymptotics}

This section deals with the proof of Theorem 1.6. The proof follows the lines of that of [4, Theorem 1.12]. We still give the proof in full, since we believe that the setting of the present article makes it accessible to a wider audience than [4]. In all this section, we consider a Gaussian process $f$ which is at least of class $\mathcal{C}^{1}$, stationary, centered and normalized. We denote by $\kappa$ is correlation function, which is assumed to tend to 0 at infinity. In particular, the process $f$ satisfies the conclusion of Lemma 2.10.

In Section 7.1, we derive an integral expression of the central moments we are interested in. In order to understand this integral, we split $\mathbb{R}^{p}$ as $\bigsqcup_{\mathcal{I} \in \mathcal{P}_{p}} \mathbb{R}_{\mathcal{I}, \eta}^{p}$, for some $\eta>0$, and study the contribution of each $\mathbb{R}_{\mathcal{I}, \eta}^{p}$ to the integral. In Section 7.2, we give an upper bound for the contribution of each of these sets. Then, in Sections 7.3 and 7.4, we study the contributions of the pieces of the form $\mathbb{R}_{\mathcal{I}, \eta}^{p}$, where respectively $\mathcal{I}$ contains a singleton and $\mathcal{I}$ is a partition into pairs. We conclude the proof of Theorem 1.6 in Section 7.5. 


\subsection{An integral expression of the central moments}

Let $R>0$, recall that $\nu_{R}$ denotes the counting measure of $Z_{R}=\{x \in \mathbb{R} \mid f(R x)=0\}$. Let $p \geqslant 2$ be an integer and let $\phi_{1}, \ldots, \phi_{p}$ be test-functions in the sense of Definition 1.2. In this section, we derive an integral expression of the quantity $m_{p}\left(\nu_{R}\right)\left(\phi_{1}, \ldots, \phi_{p}\right)$ defined by Definition 1.1. This requires to introduce the following definition.

Definition 7.1 (Subsets adapted to a partition). Let $A$ be a finite set and let $\mathcal{I} \in \mathcal{P}_{A}$, we denote by $S_{A}(\mathcal{I})$ the set of subsets of $A$ adapted to $\mathcal{I}$, that is:

$$
\mathcal{S}_{A}(\mathcal{I})=\{B \subset A \mid \forall I \in \mathcal{I} \text {, if } \operatorname{Card}(I) \geqslant 2 \text {, then } I \subset B\}
$$

Equivalently, $B \in \mathcal{S}_{A}(\mathcal{I})$ if and only if $\mathcal{I} \leqslant\{B\} \sqcup \mathcal{I}_{\min }(A \backslash B)$ where $\leqslant$ is as in Definition 6.3 and $\mathcal{I}_{\min }(A \backslash B)=\{\{b\} \mid b \notin B\}$. If $A$ is of the form $\{1, \ldots, p\}$, we simply denote by $S_{p}(\mathcal{I})=S_{A}(\mathcal{I})$

Let $A$ be a finite set and let $\mathcal{I} \in \mathcal{P}_{A}$. Let $B \in \mathcal{S}_{A}(\mathcal{I})$, we have $\mathcal{I} \leqslant\{B\} \sqcup \mathcal{I}_{\text {min }}(A \backslash B)$, so that $\mathcal{I}_{B}=\{I \in \mathcal{I} \mid I \subset B\}$ is a well-defined element of $\mathcal{P}_{B}$, as in Notation 6.5. In fact, we have:

$$
\mathcal{I}=\mathcal{I}_{B} \sqcup \mathcal{I}_{\min }(A \backslash B)
$$

Lemma 7.2. Let $A$ be any finite set, then the map $(B, \mathcal{I}) \mapsto\left(B, \mathcal{I}_{B}\right)$ defines a bijection from $\left\{(B, \mathcal{I}) \mid \mathcal{I} \in \mathcal{P}_{A}, B \in \mathcal{S}_{A}(\mathcal{I})\right\}$ to $\left\{(B, \mathcal{J}) \mid B \subset A, \mathcal{J} \in \mathcal{P}_{B}\right\}$.

Proof. This map is well-defined. By Equation (7.1), the map $(B, \mathcal{J}) \mapsto\left(B, \mathcal{J} \sqcup \mathcal{I}_{\min }(A \backslash B)\right)$ from $\left\{(B, \mathcal{J}) \mid B \subset A, \mathcal{J} \in \mathcal{P}_{B}\right\}$ to $\left\{(B, \mathcal{I}) \mid \mathcal{I} \in \mathcal{P}_{A}, B \in \mathcal{S}_{A}(\mathcal{I})\right\}$ is the inverse of $(B, \mathcal{I}) \mapsto\left(B, \mathcal{I}_{B}\right)$

For any non-empty finite set $A$, let us denote by $\mathrm{d} \underline{x}_{A}$ the Lebesgue measure on $\mathbb{R}^{A}$. The following lemma gives the integral expression of $m_{p}\left(\nu_{R}\right)\left(\phi_{1}, \ldots, \phi_{p}\right)$ we are looking for. This integral expression is a sum of integrals over $\mathbb{R}^{\mathcal{I}}$, indexed by $\mathcal{I} \in \mathcal{P}_{p}$. For each of these terms, the integrand function is itself a sum of functions that are indexed by partitions of some partitions induced by $\mathcal{I}$. Hence we need to consider partitions of partitions, which is a bit cumbersome.

Lemma 7.3 (Integral expression of the central moments). Let $p \geqslant 2$ and let us assume that $f$ is of class $\mathcal{C}^{p}$. Let $\phi_{1}, \ldots, \phi_{p}$ be test-functions in the sense of Definition 1.2. For all $R>0$, we have:

$$
m_{p}\left(\nu_{R}\right)\left(\phi_{1}, \ldots, \phi_{p}\right)=\sum_{\mathcal{I} \in \mathcal{P}_{p}} \int_{\mathbb{R}^{\mathcal{I}}} \iota_{\mathcal{I}}^{*} \phi_{R}\left(\underline{x}_{\mathcal{I}}\right) F_{\mathcal{I}}\left(\underline{x}_{\mathcal{I}}\right) \mathrm{d} \underline{x}_{\mathcal{I}}
$$

where, for any finite set $A \neq \emptyset$, any $\mathcal{I} \in \mathcal{P}_{A}$ and any $\underline{x}_{\mathcal{I}}=\left(x_{I}\right)_{I \in \mathcal{I}} \in \mathbb{R}^{\mathcal{I}}$,

$$
F_{\mathcal{I}}\left(\underline{x}_{\mathcal{I}}\right)=\sum_{B \in \mathcal{S}_{A}(\mathcal{I})}\left(\frac{-1}{\pi}\right)^{|A|-|B|} \rho_{\left\{\mathcal{I}_{B}\right\}}\left(\underline{x}_{\mathcal{I}_{B}}\right) .
$$

Here, we use the convention that $\rho_{\{\emptyset\}}$ is constant equal to 1 . Note that $\mathcal{I}_{B}=\emptyset$ if and only if $B=\emptyset$.

Proof. The proof follows the lines of [4, Lemma 3.1]. Recall that $m_{p}\left(\nu_{R}\right)$ is defined by 
Definition 1.1. We develop this product using Notations 2.1, we get:

$$
\begin{aligned}
m_{p}\left(\nu_{R}\right)\left(\phi_{1}, \ldots, \phi_{p}\right) & =\sum_{B \subset\{1, \ldots, p\}}(-1)^{p-|B|} \mathbb{E}\left[\prod_{i \in B}\left\langle\nu_{R}, \phi_{i}\right\rangle\right] \prod_{i \notin B} \mathbb{E}\left[\left\langle\nu_{R}, \phi_{i}\right\rangle\right] \\
& =\sum_{B \subset\{1, \ldots, p\}}(-1)^{p-|B|} \mathbb{E}\left[\left\langle\nu^{B},\left(\phi_{B}\right)_{R}\right\rangle\right] \prod_{i \notin B} \mathbb{E}\left[\left\langle\nu_{R}, \phi_{i}\right\rangle\right] \\
& =\sum_{B \subset\{1, \ldots, p\}} \sum_{\mathcal{I} \in \mathcal{P}_{B}}(-1)^{p-|B|} \mathbb{E}\left[\left\langle\nu^{[\mathcal{I}]}, \iota_{\mathcal{I}}^{*}\left(\left(\phi_{B}\right)_{R}\right)\right\rangle\right] \prod_{i \notin B} \mathbb{E}\left[\left\langle\nu_{R}, \phi_{i}\right\rangle\right],
\end{aligned}
$$

where the last equality comes from Lemma 2.7.

Let $B \subset\{1, \ldots, p\}$ and $\mathcal{I} \in \mathcal{P}_{B}$. Note first that $\iota_{\mathcal{I}}^{*}\left(\left(\phi_{B}\right)_{R}\right)=\phi_{B}\left(\frac{\iota_{\mathcal{I}}(\cdot)}{R}\right)=\left(\iota_{\mathcal{I}}^{*} \phi_{B}\right)_{R}$. Then, we can identify $\mathbb{R}^{\mathcal{I}}$ with $\mathbb{R}^{|\mathcal{I}|}$ by ordering $\mathcal{I}$. Since the $\left(\phi_{i}\right)_{1 \leqslant i \leqslant p}$ are integrable on $\mathbb{R}$ and essentially bounded, $\iota_{\mathcal{I}}^{*} \phi_{B}$ is integrable on $\mathbb{R}^{\mathcal{I}}$. By Propositions 3.6 and 6.23, we obtain:

$$
\begin{aligned}
\mathbb{E}\left[\left\langle\nu^{[\mathcal{I}]}, \iota_{\mathcal{I}}^{*}\left(\left(\phi_{B}\right)_{R}\right)\right\rangle\right] & \left.=\mathbb{E}\left[\left\langle\nu^{[|\mathcal{I}|]},\left(\iota_{\mathcal{I}}^{*} \phi_{B}\right) R\right)\right\rangle\right] \\
& =\int_{\mathbb{R}^{|\mathcal{I}|}} \iota_{\mathcal{I}}^{*} \phi_{B}\left(\frac{x_{1}}{R}, \ldots, \frac{x_{|\mathcal{I}|}}{R}\right) \rho_{|\mathcal{I}|}\left(x_{1}, \ldots, x_{|\mathcal{I}|}\right) \mathrm{d} x_{1} \ldots \mathrm{d} x_{|\mathcal{I}|} \\
& =\int_{\mathbb{R}^{\mathcal{I}}} \iota_{\mathcal{I}}^{*} \phi_{B}\left(\frac{\underline{x}_{\mathcal{I}}}{R}\right) \rho_{\{\mathcal{I}\}}\left(\underline{x}_{\mathcal{I}}\right) \mathrm{d} \underline{x}_{\mathcal{I}},
\end{aligned}
$$

where $\rho_{\{\mathcal{I}\}}: \mathbb{R}^{\mathcal{I}} \rightarrow \mathbb{R}$ is defined by Equation (6.4). As in Section 3.3, for any $i \notin B$, we have $\mathbb{E}\left[\left\langle\nu_{R}, \phi_{i}\right\rangle\right]=\frac{1}{\pi} \int_{\mathbb{R}} \phi_{i}\left(\frac{x}{R}\right) \mathrm{d} x$. Hence,

$$
\begin{aligned}
& m_{p}\left(\nu_{R}\right)\left(\phi_{1}, \ldots, \phi_{p}\right)= \\
& \sum_{B \subset\{1, \ldots, p\}} \sum_{\mathcal{I} \in \mathcal{P}_{B}}\left(\frac{-1}{\pi}\right)^{p-|B|} \int_{\mathbb{R}^{\mathcal{I}}} \iota_{\mathcal{I}}^{*} \phi_{B}\left(\frac{\underline{x}_{\mathcal{I}}}{R}\right) \rho_{\{\mathcal{I}\}}\left(\underline{x}_{\mathcal{I}}\right) \mathrm{d} \underline{x}_{\mathcal{I}} \prod_{i \notin B} \int_{\mathbb{R}} \phi_{i}\left(\frac{x}{R}\right) \mathrm{d} x .
\end{aligned}
$$

By Lemma 7.2, we can exchange the two sums and obtain the following:

$$
\sum_{\mathcal{I} \in \mathcal{P}_{p}} \sum_{B \in \mathcal{S}_{p}(\mathcal{I})}\left(\frac{-1}{\pi}\right)^{p-|B|}\left(\int_{\mathbb{R}^{\mathcal{I}_{B}}} \iota_{\mathcal{I}_{B}}^{*} \phi_{B}\left(\frac{\underline{x}_{\mathcal{I}_{B}}}{R}\right) \rho_{\left\{\mathcal{I}_{B}\right\}}\left(\underline{x}_{\mathcal{I}_{B}}\right) \mathrm{d} \underline{x}_{\mathcal{I}_{B}}\right) \prod_{i \notin B} \int_{\mathbb{R}} \phi_{i}\left(\frac{x}{R}\right) \mathrm{d} x .
$$

We conclude the proof by applying Fubini's Theorem, which yields:

$$
m_{p}\left(\nu_{R}\right)\left(\phi_{1}, \ldots, \phi_{p}\right)=\sum_{\mathcal{I} \in \mathcal{P}_{p}} \int_{\mathbb{R}^{\mathcal{I}}} \iota_{\mathcal{I}}^{*} \phi_{R}\left(\underline{x}_{\mathcal{I}}\right)\left(\sum_{B \in \mathcal{S}_{p}(\mathcal{I})}\left(\frac{-1}{\pi}\right)^{p-|B|} \rho_{\left\{\mathcal{I}_{B}\right\}}\left(\underline{x}_{\mathcal{I}_{B}}\right)\right) \mathrm{d} \underline{x}_{\mathcal{I}} .
$$

Example 7.4. Let $A=\{a, b\}$, then $\mathcal{P}_{A}$ contains only two elements: $\mathcal{I}_{\text {min }}(A)=\{\{a\},\{b\}\}$ and $\mathcal{I}_{\max }(A)=\{A\}$. In the first case, $\mathcal{S}_{A}\left(\mathcal{I}_{\min }(A)\right)=\{\emptyset,\{a\},\{b\}, A\}$, and one can check that, $F_{\{\{a\},\{b\}\}}:(x, y) \mapsto \rho_{\left\{\mathcal{I}_{\min }(A)\right\}}(x, y)-\frac{1}{\pi^{2}}$. In fact, for all $(x, y) \in \mathbb{R}^{2} \backslash \Delta_{2}$, we have:

$$
F_{\{\{a\},\{b\}\}}(x, y)=\rho_{2}(x, y)-\frac{1}{\pi^{2}}=F(y-x),
$$

where $F$ is as in Definition 4.1. In the second case, $\mathcal{S}_{A}(\{A\})=\{A\}$ and $F_{\{A\}}=\frac{1}{\pi}$. Then, if $\phi_{a}$ and $\phi_{b}$ are integrable and $\phi_{b}$ is bounded and continuous almost everywhere, we have:

$$
\int_{\mathbb{R}\{A\}}\left(\phi_{a}\right)_{R}(x)\left(\phi_{b}\right)_{R}(x) F_{\{A\}}(x) \mathrm{d} x=\frac{R}{\pi} \int_{\mathbb{R}} \phi_{a}(x) \phi_{b}(x) \mathrm{d} x .
$$


Moreover, the computations of Section 4.1 show that:

$$
\begin{aligned}
\int_{\mathbb{R}\{\{a\},\{b\}\}}\left(\phi_{a}\right)_{R}(x)\left(\phi_{b}\right)_{R}(y) F_{\{\{a\},\{b\}\}}(x, y) \mathrm{d} x \mathrm{~d} y & =\int_{\mathbb{R}^{2}}\left(\phi_{a}\right)_{R}(x)\left(\phi_{b}\right)_{R}(y) F(y-x) \mathrm{d} x \mathrm{~d} y \\
& =R\left(\sigma^{2}-\frac{1}{\pi}\right) \int_{\mathbb{R}^{2}} \phi_{a}(x) \phi_{b}(x) \mathrm{d} x+o(R),
\end{aligned}
$$

where $\sigma^{2}$ is defined by Equation (1.2).

Lemma 7.5 (Boundedness). Let $A$ be a finite set. Let us assume that $f$ is of class $\mathcal{C}^{|A|}$ and that $\|\kappa\|_{|A|, \eta} \underset{\eta \rightarrow+\infty}{\longrightarrow} 0$. Then, for all $\mathcal{I} \in \mathcal{P}_{A}$, the function $F_{\mathcal{I}}$ is bounded on $\mathbb{R}^{\mathcal{I}}$.

Proof. Let $B \in \mathcal{S}_{A}(\mathcal{I})$. Since $\mathcal{I}_{B} \in \mathcal{P}_{B}$, we have $\left|\mathcal{I}_{B}\right| \leqslant|B| \leqslant|A|$. Thus $\|\kappa\|_{\left|\mathcal{I}_{B}\right|, \eta} \underset{\eta \rightarrow+\infty}{\longrightarrow} 0$, and $\rho_{\left\{\mathcal{I}_{B}\right\}}$ is bounded by Lemma 6.41. The conclusion follows from the expression of $F_{\mathcal{I}}$ given by Equation (7.2).

In order to compute the central moments of $\nu_{R}$, we have to estimate the integral of $F_{\mathcal{I}}$ over $\mathbb{R}^{\mathcal{I}}$ for any partition $\mathcal{I} \in \mathcal{P}_{p}$. This is done by writing $\mathbb{R}^{\mathcal{I}}$ as $\bigsqcup_{\mathcal{J} \in \mathcal{P}_{\mathcal{I}}} \mathbb{R}_{\mathcal{I}, \eta}^{\mathcal{I}}$ for some well-chosen $\eta>0$ and proving estimates for the contribution of each $\mathbb{R}_{\mathcal{J}, \eta}^{\mathcal{I}}$, In the following sections, we will derive estimates for $F_{\mathcal{I}}$ on $\mathbb{R}_{\mathcal{J}, \eta}^{\mathcal{I}}$, depending on the combinatorial properties of the partitions $\mathcal{I}$ and $\mathcal{J}$.

Let us conclude this section by choosing the scale parameter $\eta$. In the following, we will work, not with a fixed $\eta>0$, but with a scale parameter depending on $R$, given by the following lemma.

Lemma 7.6 (Scale parameter). Let $p \geqslant 2$ be an integer. Let us assume that $f$ is of $\operatorname{class} \mathcal{C}^{p}$ and that $\|\kappa\|_{p, \eta}=o\left(\eta^{-4 p}\right)$ as $\eta \rightarrow+\infty$. Then, there exists a function $\eta:(0,+\infty) \rightarrow(0,+\infty)$ such that, as $R \rightarrow+\infty$ we have: $\eta(R) \rightarrow+\infty, \eta(R)=o\left(R^{\frac{1}{4}}\right)$ and $\|\kappa\|_{p, \eta(R)}=o\left(R^{-p}\right)$.

Proof. Let $\varepsilon: \tau \mapsto \tau^{4 p}\|\kappa\|_{p, \tau}$ from $[0,+\infty)$ to itself. We have $\varepsilon(\tau) \rightarrow 0$ as $\tau \rightarrow+\infty$ since $\|\kappa\|_{p, \tau}=o\left(\tau^{-4 p}\right)$. Note that this implies that $\varepsilon$ is bounded on $[0,+\infty)$. For all $R>0$ we denote by $\alpha(R)=\max \left(R^{-\frac{1}{8}},\left(\sup \left\{\varepsilon(\tau) \mid \tau \geqslant R^{\frac{1}{8}}\right\}\right)^{\frac{1}{8 p}}\right)$. Since $\varepsilon(\tau) \underset{\tau \rightarrow+\infty}{\longrightarrow} 0$ at infinity, we have $\alpha(R) \underset{R \rightarrow+\infty}{\longrightarrow} 0$. Let us set $\eta(R)=R^{\frac{1}{4}} \alpha(R)$ for all $R>0$, and let us check that $\eta$ satisfies the desired conditions.

Since $\alpha$ goes to 0 at infinity, we have $\eta(R)=o\left(R^{\frac{1}{4}}\right)$ as $R \rightarrow+\infty$. Besides, for all $R>0$ we have $\alpha(R) \geqslant R^{-\frac{1}{8}}$, hence $\eta(R) \geqslant R^{\frac{1}{8}}$ and $\eta(R) \underset{R \rightarrow+\infty}{\longrightarrow}+\infty$. Then, let $R>0$, we have:

$$
\|\kappa\|_{p, \eta(R)}=\frac{\varepsilon(\eta(R))}{\eta(R)^{4 p}}=\frac{1}{R^{p}} \frac{\varepsilon(\eta(R))}{\alpha(R)^{4 p}} .
$$

Since $\eta(R) \geqslant R^{\frac{1}{8}}$, we have $\varepsilon(\eta(R)) \leqslant \sup \left\{\varepsilon(\tau) \mid \tau \geqslant R^{\frac{1}{8}}\right\} \leqslant \alpha(R)^{8 p}$. Finally,

$$
\|\kappa\|_{p, \eta(R)} \leqslant \frac{\alpha(R)^{4 p}}{R^{p}}=o\left(R^{-p}\right) .
$$

\subsection{An upper bound on the contribution of each piece}

In this section, we give upper bounds for the contribution of each addend in the expression of $m_{p}\left(\nu_{R}\right)\left(\phi_{1}, \ldots, \phi_{p}\right)$ derived in Lemma 7.3. We bound the contribution of the integral of the term indexed by $\mathcal{I} \in \mathcal{P}_{p}$ over $\mathbb{R}_{\mathcal{J}, \eta(R)}^{\mathcal{I}}$ in terms of the combinatorial properties of $\mathcal{I}$ and $\mathcal{J}$. See Lemma 7.9 below for a precise statement. 
Remark 7.7. Note that $\mathcal{J}$ is partition of $\mathcal{I}$, which is itself a partition of $\{1, \ldots, p\}$. This is the main reason why our formalism is so heavy. A good starting point is to understand what happens for the term indexed by $\mathcal{I}=\mathcal{I}_{\min }(p)=\{\{i\} \mid 1 \leqslant i \leqslant p\}$ in Lemma 7.3. In this case, all the important ideas of the proof appear, but we can simplify the formalism a bit since $\mathcal{I} \simeq\{1, \ldots, p\}$ canonically.

Lemma 7.8. Let $A$ be a non-empty finite set and let us assume that $f$ is a $\mathcal{C}^{|A|}$-process such that $\|\kappa\|_{|A|, \eta}=o\left(\eta^{-4|A|}\right)$ at infinity. Let $\eta:[0,+\infty) \rightarrow[0,+\infty)$ be a function satisfying the conditions of Lemma 7.6 with $p=|A|$.

Let $\mathcal{I} \in \mathcal{P}_{A}$ and let $S \subset A$ be of even cardinality and such that $\mathcal{I}_{S}=\{\{s\} \mid s \in S\} \subset \mathcal{I}$. Let $\mathcal{J}^{\prime} \in \mathcal{P P}_{\mathcal{I}_{S}}$ and $\mathcal{J}^{\prime \prime} \in \mathcal{P}_{\mathcal{I} \backslash \mathcal{I}_{S}}$, we denote by $\mathcal{J}=\mathcal{J}^{\prime} \sqcup \mathcal{J}^{\prime \prime} \in \mathcal{P}_{\mathcal{I}}$. Then, the following holds uniformly for all $\underline{x}_{\mathcal{I}} \in \mathbb{R}_{\mathcal{J}, \eta(R)}^{\mathcal{I}}$ :

$$
F_{\mathcal{I}}\left(\underline{x}_{\mathcal{I}}\right)=F_{\mathcal{I} \backslash \mathcal{I}_{S}}\left(\underline{x}_{\mathcal{I} \backslash \mathcal{I}_{S}}\right) \prod_{J \in \mathcal{J}^{\prime}} F_{J}\left(\underline{x}_{J}\right)+o\left(R^{-\frac{|A|}{2}}\right) .
$$

Proof. If $S$ is empty, then $\mathcal{I}_{S}=\emptyset$ and $\mathcal{J}^{\prime}=\emptyset$ by convention. Hence, the result holds in this case. Let us now assume that $S$ is not empty. Then $\mathcal{I}_{S}$ is non-empty and contains an even number of elements, so that $\mathcal{P P}_{\mathcal{I}_{S}}$ is non-empty.

Let $\mathcal{J}^{\prime} \in \mathcal{P P}_{\mathcal{I}_{S}}$, let $\mathcal{J}^{\prime \prime} \in \mathcal{P}_{\mathcal{I} \backslash \mathcal{I}_{S}}$ and let $\mathcal{J}=\mathcal{J}^{\prime} \sqcup \mathcal{J}^{\prime \prime}$. Let $J \in \mathcal{J}^{\prime}$, then there exists $s$ and $t \in S$ such that $s \neq t$ and $J=\{\{s\},\{t\}\}$. Let us denote by $A^{\prime}=A \backslash\{s, t\}$, we have $\mathcal{I} \leqslant\left\{A^{\prime},\{s\},\{t\}\right\}$ so that $\mathcal{I}=\mathcal{I}_{A^{\prime}} \sqcup\{\{s\},\{t\}\}$. Recall that $F_{\mathcal{I}}$ is defined as a sum indexed by $B \in \mathcal{S}_{A}(\mathcal{I})$, see Equation (7.2). Since $\{s\}$ and $\{t\} \in \mathcal{I}$, we have:

$$
\mathcal{S}_{A}(\mathcal{I})=\bigsqcup_{B \in \mathcal{S}_{A^{\prime}}\left(\mathcal{I}_{A^{\prime}}\right)}\{B, B \sqcup\{s\}, B \sqcup\{t\}, B \sqcup\{s, t\}\} .
$$

Let $B \in S_{A^{\prime}}\left(\mathcal{I}_{A^{\prime}}\right)$, we regroup the four terms corresponding to $B, B \sqcup\{s\}, B \sqcup\{t\}$ and $B \sqcup\{s, t\}$ in the sum defining $F_{\mathcal{I}}$ (see Lemma 7.3). For all $\underline{x}_{\mathcal{I}} \in \mathbb{R}^{\mathcal{I}}$, we obtain:

$$
\begin{aligned}
& \left(\frac{-1}{\pi}\right)^{|A|-|B|}\left(\pi^{2} \rho_{\left\{\mathcal{I}_{B \sqcup\{s, t\}}\right\}}\left(\underline{x}_{\mathcal{I}_{B \sqcup\{s, t\}}}\right)-\pi \rho_{\left\{\mathcal{I}_{B \sqcup\{s\}}\right\}}\left(\underline{x}_{\mathcal{I}_{B \sqcup\{s\}}}\right)\right. \\
& \left.-\pi \rho_{\left\{\mathcal{I}_{B \sqcup\{t\}}\right\}}\left(\underline{x}_{\mathcal{I}_{B \sqcup\{t\}}}\right)+\rho_{\left\{\mathcal{I}_{B}\right\}}\left(\underline{x}_{\mathcal{I}_{B}}\right)\right) .
\end{aligned}
$$

Note that $\mathcal{I}_{B \sqcup\{s, t\}}=\mathcal{I}_{B} \sqcup J$. Since $J=\{\{s\},\{t\}\} \in \mathcal{J}$, if $\underline{x}_{\mathcal{I}} \in \mathbb{R}_{\mathcal{J}, \eta(R)}^{\mathcal{I}}$, then for any $I \in \mathcal{I} \backslash J$ we have $\left|x_{I}-x_{\{s\}}\right| \geqslant \eta(R)$ and $\left|x_{I}-x_{\{t\}}\right| \geqslant \eta(R)$. In particular, the following holds:

$$
\mathcal{I}_{\eta(R)}\left(\underline{x}_{\mathcal{I}_{B} \sqcup J}\right)=\mathcal{J}_{\mathcal{I}_{B} \sqcup J} \leqslant\left\{\mathcal{I}_{B}, J\right\} .
$$

Since we chose $\eta$ so that $\|\kappa\|_{|A|, \eta(R)}=o\left(R^{-|A|}\right)$, applying Corollary 6.44 we get:

$$
\rho_{\left\{\mathcal{I}_{B \sqcup\{s, t\}}\right\}}\left(\underline{x}_{\mathcal{I}_{B \sqcup\{s, t\}}}\right)=\rho_{\left\{\mathcal{I}_{B} \sqcup J\right\}}\left(\underline{x}_{\mathcal{I}_{B} \sqcup J}\right)=\rho_{\left\{\mathcal{I}_{B}\right\}}\left(\underline{x}_{\mathcal{I}_{B}}\right) \rho_{\{J\}}\left(\underline{x}_{J}\right)\left(1+o\left(R^{-\frac{|A|}{2}}\right)\right) .
$$

We proceed similarly with the three other terms in Equation (7.4). Bearing in mind that $\rho_{\{J\}}$ and $\rho_{\left\{\mathcal{I}_{B}\right\}}$ are bounded (see Lemma 6.41), we obtain that (7.4) equals:

$$
\left(\frac{-1}{\pi}\right)^{|A|-|B|-2} \rho_{\left\{\mathcal{I}_{B}\right\}}\left(\underline{x}_{\mathcal{I}_{B}}\right) F_{J}\left(\underline{x}_{J}\right)+o\left(R^{-\frac{|A|}{2}}\right) .
$$

Summing these terms over $B \subset A^{\prime}$, we get that, for all $\underline{x}_{\mathcal{I}} \in \mathbb{R}_{\mathcal{J}, \eta(R)}^{\mathcal{I}}$ :

$$
F_{\mathcal{I}}\left(\underline{x}_{\mathcal{I}}\right)=F_{\mathcal{I} \backslash J}\left(\underline{x}_{\mathcal{I} \backslash J}\right) F_{J}\left(\underline{x}_{J}\right)+o\left(R^{-\frac{|A|}{2}}\right) .
$$

We can repeat the argument for $F_{\mathcal{I} \backslash J}\left(\underline{x}_{\mathcal{I} \backslash J}\right)$, with $\mathcal{J}^{\prime}$ replaced by $\mathcal{J}^{\prime} \backslash\{J\}$. More formally, we prove by induction on the cardinality of $\mathcal{J}^{\prime}$ that Equation (7.3) holds uniformly on $\mathbb{R}_{\mathcal{J}, \eta(R)}^{\mathcal{I}}$. The result is true if $\mathcal{J}^{\prime}=\emptyset$, and the inductive step is given by Equation (7.5). 
Lemma 7.9. In the same setting as Lemma 7.8, let $\left(\phi_{a}\right)_{a \in A}$ be Lebesgue-integrable and essentially bounded functions. Then, as $R \rightarrow+\infty$, we have:

$$
\int_{\mathbb{R}_{\mathcal{J}, \eta(R)}^{\mathcal{I}}} \iota_{\mathcal{I}}^{*} \phi_{R}\left(\underline{x}_{\mathcal{I}}\right) F_{\mathcal{I}}\left(\underline{x}_{\mathcal{I}}\right) \mathrm{d} \underline{x}_{\mathcal{I}}=O\left(R^{|\mathcal{J}|} \eta(R)^{|\mathcal{I}|-2\left|\mathcal{J}^{\prime}\right|-\left|\mathcal{J}^{\prime \prime}\right|}\right),
$$

where $\eta:[0,+\infty) \rightarrow[0,+\infty)$ is a function satisfying the conditions of Lemma 7.6.

Proof. Let $\underline{x}_{I}=\left(x_{I}\right)_{I \in \mathcal{I}} \in \mathbb{R}_{\mathcal{J}, \eta(R)}^{\mathcal{I}}$, using the estimate of Lemma 7.8, we have:

$$
\iota_{\mathcal{I}}^{*} \phi_{R}\left(\underline{x}_{\mathcal{I}}\right) F_{\mathcal{I}}\left(\underline{x}_{\mathcal{I}}\right)=\iota_{\mathcal{I}}^{*} \phi_{R}\left(\underline{x}_{\mathcal{I}}\right) F_{\mathcal{I} \backslash \mathcal{I}_{S}}\left(\underline{x}_{\mathcal{I} \backslash \mathcal{I}_{S}}\right) \prod_{J \in \mathcal{J}^{\prime}} F_{J}\left(\underline{x}_{J}\right)+o\left(R^{-\frac{|A|}{2}}\right) \iota_{\mathcal{I}}^{*} \phi_{R}\left(\underline{x}_{\mathcal{I}}\right) \text {. }
$$

Then, since $\mathcal{I}=\left(\mathcal{I} \backslash \mathcal{I}_{S}\right) \sqcup \bigsqcup_{J \in \mathcal{J}^{\prime}} J$, by Fubini's Theorem,

$$
\begin{aligned}
\left|\int_{\mathbb{R}_{\mathcal{J}, \eta(R)}^{\mathcal{I}}} \iota_{\mathcal{I}}^{*} \phi_{R}\left(\underline{x}_{\mathcal{I}}\right) F_{\mathcal{I} \backslash \mathcal{I}_{S}}\left(\underline{x}_{\mathcal{I} \backslash \mathcal{I}_{S}}\right) \prod_{J \in \mathcal{J}^{\prime}} F_{J}\left(\underline{x}_{J}\right) \mathrm{d} \underline{x}_{\mathcal{I}}\right| \\
\leqslant \int_{\mathbb{R}_{\mathcal{I}, \eta(R)}^{\mathcal{I}} \mid}\left|\iota_{\mathcal{I}}^{*} \phi_{R}\left(\underline{x}_{\mathcal{I}}\right) F_{\mathcal{I} \backslash \mathcal{I}_{S}}\left(\underline{x}_{\mathcal{I} \backslash \mathcal{I}_{S}}\right) \prod_{J \in \mathcal{J}^{\prime}} F_{J}\left(\underline{x}_{J}\right)\right| \mathrm{d} \underline{x}_{\mathcal{I}} \\
\leqslant \int_{\mathbb{R}_{\mathcal{J}^{\prime \prime}, \eta(R)}^{\mathcal{I} \backslash \mathcal{I}_{S}}}\left|F_{\mathcal{I} \backslash \mathcal{I}_{S}}\left(\underline{x}_{\mathcal{I} \backslash \mathcal{I}_{S}}\right)\right| \prod_{I \notin \mathcal{I}_{S}} \prod_{i \in I}\left|\phi_{i}\left(\frac{x_{I}}{R}\right)\right| \mathrm{d} \underline{x}_{\mathcal{I} \backslash \mathcal{I}_{S}} \\
\quad \times \prod_{J \in \mathcal{J}^{\prime}} \int_{\mathbb{R}^{J}}\left|F_{J}\left(\underline{x}_{J}\right)\right| \prod_{I \in J} \prod_{i \in I}\left|\phi_{i}\left(\frac{x_{I}}{R}\right)\right| \mathrm{d} \underline{x}_{J},
\end{aligned}
$$

where we used the fact that $\mathbb{R}_{\mathcal{J}, \eta(R)}^{\mathcal{I}} \subset \mathbb{R}_{\mathcal{J}^{\prime \prime}, \eta(R)}^{\mathcal{I} \backslash \mathcal{I}_{S}} \times \mathbb{R}^{\mathcal{I}_{S}}=\mathbb{R}_{\mathcal{J}^{\prime \prime}, \eta(R)}^{\mathcal{I} \backslash \mathcal{I}_{S}} \times \prod_{J \in \mathcal{J}^{\prime}} \mathbb{R}^{J}$. The same kind of computation shows that:

$$
\begin{aligned}
\left|\int_{\mathbb{R}_{\mathcal{J}, \eta(R)}^{\mathcal{I}}} \iota_{\mathcal{I}}^{*} \phi_{R}\left(\underline{x}_{\mathcal{I}}\right) \mathrm{d} \underline{x}_{\mathcal{I}}\right| \leqslant \int_{\mathbb{R}_{\mathcal{J}^{\prime \prime}, \eta(R)}^{\mathcal{I} \backslash \mathcal{I}_{S}}} \prod_{I \notin \mathcal{I}_{S}} \prod_{i \in I}\left|\phi_{i}\left(\frac{x_{I}}{R}\right)\right| \mathrm{d} \underline{x}_{\mathcal{I} \backslash \mathcal{I}_{S}} \\
\times \prod_{J \in \mathcal{J}^{\prime}} \int_{\mathbb{R}^{J}} \prod_{I \in J} \prod_{i \in I}\left|\phi_{i}\left(\frac{x_{I}}{R}\right)\right| \mathrm{d} \underline{x}_{J} .
\end{aligned}
$$

Let $J=\{\{a\},\{b\}\} \in \mathcal{J}^{\prime}$. Since $\phi_{a}$ and $\phi_{b}$ are integrable on $\mathbb{R}$, then $\phi_{a} \otimes \phi_{b}$ is integrable on $\mathbb{R}^{2}$. Then, as explained in Example 7.4, we have:

$$
\int_{\mathbb{R}^{J}}\left|F_{J}\left(\underline{x}_{J}\right)\right| \prod_{I \in J} \prod_{i \in I}\left|\phi_{i}\left(\frac{x_{I}}{R}\right)\right| \mathrm{d} \underline{x}_{J}=\int_{\mathbb{R}^{2}}\left|\phi_{a}\left(\frac{x}{R}\right) \phi_{b}\left(\frac{y}{R}\right)\right||F(y-x)| \mathrm{d} x \mathrm{~d} y,
$$

where $F$ is defined by Definition 4.1. Then, recall that $\phi_{a}$ and $\phi_{b}$ are essentially bounded. Denoting by $\left\|\phi_{b}\right\|_{\infty}$ the essential supremum of $\phi_{b}$, we have:

$$
\begin{aligned}
\int_{\mathbb{R}^{2}}\left|\phi_{a}\left(\frac{x}{R}\right) \phi_{b}\left(\frac{y}{R}\right)\right||F(y-x)| \mathrm{d} x \mathrm{~d} y & \leqslant\left\|\phi_{b}\right\|_{\infty} \int_{\mathbb{R}^{2}}\left|\phi_{a}\left(\frac{x}{R}\right)\right||F(z)| \mathrm{d} x \mathrm{~d} z \\
& \leqslant R\left\|\phi_{b}\right\|_{\infty}\left(\int_{\mathbb{R}}\left|\phi_{a}(x)\right| \mathrm{d} x\right)\left(\int_{\mathbb{R}}|F(z)| \mathrm{d} z\right) .
\end{aligned}
$$

Since $\phi_{a}$ and $F$ are integrable (see Lemma 4.3), the previous term is $O(R)$. Hence, by Equations (7.6) and (7.8), the term on the first line of Equation (7.6) equals:

$$
O\left(R^{\left|\mathcal{J}^{\prime}\right|}\right) \int_{\mathbb{R}_{\mathcal{J}^{\prime \prime}, \eta(R)}^{\mathcal{I} \backslash \mathcal{I}_{S}}} \prod_{I \notin \mathcal{I}_{S}} \prod_{i \in I}\left|\phi_{i}\left(\frac{x_{I}}{R}\right)\right| \mathrm{d} \underline{x}_{\mathcal{I} \backslash \mathcal{I}_{S}},
$$


where we also used the boundedness of $F_{\mathcal{I} \backslash \mathcal{I}_{S}}$ on $\mathbb{R}^{\mathcal{I} \backslash \mathcal{I}_{S}}$ (see Lemma 7.5). On the other hand, we have:

$$
\int_{\mathbb{R}^{J}} \prod_{i \in I}\left|\phi_{i}\left(\frac{x_{I}}{R}\right)\right| \mathrm{d} \underline{x}_{J}=R^{2} \int_{\mathbb{R}^{2}}\left|\phi_{a}(x) \phi_{b}(y)\right| \mathrm{d} x \mathrm{~d} y=O\left(R^{2}\right),
$$

so that, by Equation (7.7),

$$
o\left(R^{-\frac{|A|}{2}}\right)\left|\int_{\mathbb{R}_{\mathcal{J}, \eta(R)}^{\mathcal{I}}} \iota_{\mathcal{I}}^{*} \phi_{R}\left(\underline{x}_{\mathcal{I}}\right) \mathrm{d} \underline{x}_{\mathcal{I}}\right|=o\left(R^{2\left|\mathcal{J}^{\prime}\right|-\frac{|A|}{2}}\right) \int_{\mathbb{R}_{\mathcal{J}^{\prime \prime}, \eta(R)}^{I} \mathcal{I}_{S}} \prod_{I \notin \mathcal{I}_{S}} \prod_{i \in I}\left|\phi_{i}\left(\frac{x_{I}}{R}\right)\right| \mathrm{d} \underline{x}_{\mathcal{I} \backslash \mathcal{I}_{S}} .
$$

Since $\mathcal{J}^{\prime} \in \mathcal{P P}_{\mathcal{I}_{S}}$ and $\mathcal{I} \in \mathcal{P}_{A}$, we have $\left|\mathcal{J}^{\prime}\right|=\frac{\left|\mathcal{I}_{S}\right|}{2} \leqslant \frac{|\mathcal{I}|}{2} \leqslant \frac{|A|}{2}$. Hence, in order to conclude the proof, it is enough to prove that:

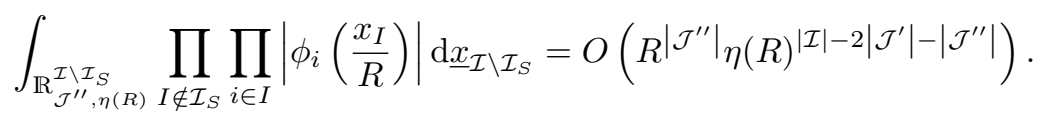

Note that $\mathbb{R}_{\mathcal{J}^{\prime \prime}, \eta(R)}^{\mathcal{I} \backslash \mathcal{I}_{S}} \subset \prod_{J \in \mathcal{J}^{\prime \prime}} \mathbb{R}_{\{J\}, \eta(R)}^{J}$. Hence, we have:

$$
\int_{\mathbb{R}_{\mathcal{J}^{\prime \prime}, \eta(R)}^{\mathcal{I} \backslash \mathcal{I}_{S}}} \prod_{I \notin \mathcal{I}_{S}} \prod_{i \in I}\left|\phi_{i}\left(\frac{x_{I}}{R}\right)\right| \mathrm{d} \underline{x}_{\mathcal{I} \backslash \mathcal{I}_{S}} \leqslant \prod_{J \in \mathcal{J}^{\prime \prime}} \int_{\mathbb{R}_{\{J\}, \eta(R)}^{J}} \prod_{I \in J} \prod_{i \in I}\left|\phi_{i}\left(\frac{x_{I}}{R}\right)\right| \mathrm{d} \underline{x}_{J}
$$

Let $J \in \mathcal{J}^{\prime \prime}$ and let $B=\bigsqcup_{I \in J} I$. Recall that, for all $a \in B$, the function $\phi_{a}$ is essentially bounded and let us denote by $\left\|\phi_{a}\right\|_{\infty}$ its essential supremum. Let us choose a preferred element $b \in B$ and let $I_{0} \in J$ be defined by $b \in I_{0}$. Then, we have:

$$
\int_{\mathbb{R}_{\{J\}, \eta(R)}^{J}} \prod_{I \in J} \prod_{i \in I}\left|\phi_{i}\left(\frac{x_{I}}{R}\right)\right| \mathrm{d} \underline{x}_{J} \leqslant\left(\prod_{a \in B \backslash\{b\}}\left\|\phi_{a}\right\|_{\infty}\right) \int_{\mathbb{R}_{\{J\}, \eta(R)}^{J}}\left|\phi_{b}\left(\frac{x_{I_{0}}}{R}\right)\right| \mathrm{d} \underline{x}_{J} .
$$

Let $\underline{x}_{J} \in \mathbb{R}_{\{J\}, \eta(R)}^{J}$, for all $I \in J \backslash\left\{I_{0}\right\}$, we have $\left|x_{I}-x_{I_{0}}\right| \leqslant|J| \eta(R)$ (cf. Remark 6.8). Thus,

$$
\begin{aligned}
\int_{\mathbb{R}_{\{J\}, \eta(R)}^{J}}\left|\phi_{b}\left(\frac{x_{I_{0}}}{R}\right)\right| \mathrm{d} \underline{x}_{J} & \leqslant(|J| \eta(R))^{|J|-1} \int_{\mathbb{R}}\left|\phi_{b}\left(\frac{x}{R}\right)\right| \mathrm{d} x \\
& =R(|J| \eta(R))^{|J|-1} \int_{\mathbb{R}}\left|\phi_{b}(x)\right| \mathrm{d} x,
\end{aligned}
$$

and this term is $O\left(R \eta(R)^{|J|-1}\right)$ since $\phi_{b}$ is integrable. Finally, by Equations (7.10), (7.11) and (7.12), we obtain:

$$
\begin{aligned}
\int_{\mathbb{R}_{\mathcal{J}^{\prime \prime}, \eta(R)}^{\mathcal{I}^{\prime} \mathcal{I}_{S}}} \prod_{I \notin \mathcal{I}_{S}} \prod_{i \in I}\left|\phi_{i}\left(\frac{x_{I}}{R}\right)\right| \mathrm{d} \underline{x}_{\mathcal{I} \backslash \mathcal{I}_{S}} & =\prod_{J \in \mathcal{J}^{\prime \prime}} O\left(R \eta(R)^{|J|-1}\right) \\
& =O\left(\left.R^{\mid \mathcal{J}^{\prime \prime}}\right|_{\eta(R)^{\sum_{J \in \mathcal{J}^{\prime \prime}}|J|-\left|\mathcal{J}^{\prime \prime}\right|}}\right) .
\end{aligned}
$$

Since $\mathcal{J}^{\prime \prime} \in \mathcal{P}_{\mathcal{I} \backslash \mathcal{I}_{S}}$, we have $\sum_{J \in \mathcal{J}^{\prime \prime}}|J|=\left|\mathcal{I} \backslash \mathcal{I}_{S}\right|=|\mathcal{I}|-\left|\mathcal{I}_{S}\right|$. Finally, since $\mathcal{J}^{\prime} \in \mathcal{P P}_{\mathcal{I}_{S}}$, we have $\left|\mathcal{I}_{S}\right|=2\left|\mathcal{J}^{\prime}\right|$. Thus, $\sum_{J \in \mathcal{J}^{\prime \prime}}|J|-\left|\mathcal{J}^{\prime \prime}\right|=|\mathcal{I}|-2\left|\mathcal{J}^{\prime}\right|-\left|\mathcal{J}^{\prime \prime}\right|$, and we just proved Equation (7.9), which yields the result.

\subsection{Contribution of the partitions with an isolated point}

The result of Lemma 7.9 may lead to think that the main contribution in the integral over $\mathbb{R}^{\mathcal{I}}$ appearing in Lemma 7.3 comes from the subsets of the form $\mathbb{R}_{\mathcal{J}, \eta(R)}^{\mathcal{I}}$, where $|\mathcal{J}|$ is large. In this section, we prove that the function $F_{\mathcal{I}}$ is uniformly small over $\mathbb{R}_{\mathcal{J}, \eta(R)}^{\mathcal{I}}$ if $\mathcal{J}$ contains a singleton. In particular, the contribution of $\mathbb{R}_{\mathcal{J}, \eta(R)}^{\mathcal{I}}$ to the integral over $\mathbb{R}^{\mathcal{I}}$ appearing in Lemma 7.3 is small if $|\mathcal{J}|>\frac{|\mathcal{I}|}{2}$. 
Lemma 7.10. Let $A$ be a finite set of cardinality at least 2 , and let us assume that $f$ is a $\mathcal{C}^{|A|}$-process such that $\|\kappa\|_{|A|, \eta}=o\left(\eta^{-4|A|}\right)$ at infinity. Let $\eta:[0,+\infty) \rightarrow[0,+\infty)$ be a function satisfying the conditions of Lemma 7.6 with $p=|A|$.

Let $\mathcal{I} \in \mathcal{P}_{A}$, we assume that there exists $i \in A$ such that $\{i\} \in \mathcal{I}$. Then, for all $\mathcal{J} \in \mathcal{P}_{\mathcal{I}}$ such that $\{\{i\}\} \in \mathcal{J}$, we have $F_{\mathcal{I}}\left(\underline{x}_{\mathcal{I}}\right)=o\left(R^{-\frac{|A|}{2}}\right)$ as $R \rightarrow+\infty$, uniformly in $\underline{x}_{\mathcal{I}} \in \mathbb{R}_{\mathcal{J}, \eta(R)}^{\mathcal{I}}$.

Proof. Recall that we defined $\mathcal{S}_{A}(\mathcal{I})$ in Definition 7.1. Let $A^{\prime}=A \backslash\{i\}$. Since $\{i\} \in \mathcal{I}$, we have $\mathcal{I}=\mathcal{I}_{A^{\prime}} \sqcup\{\{i\}\}$, and:

$$
\mathcal{S}_{A}(\mathcal{I})=\bigsqcup_{B \in \mathcal{S}_{A^{\prime}}\left(\mathcal{I}_{A^{\prime}}\right)}\{B, B \sqcup\{i\}\}
$$

Thus, we can split $\mathcal{S}_{A}(\mathcal{I})$ into the subsets that contain $i$ and those that do not, and $B \mapsto B \sqcup\{i\}$ is a bijection from $\mathcal{S}_{A^{\prime}}\left(\mathcal{I}_{A^{\prime}}\right)=\left\{B \in \mathcal{S}_{A}(\mathcal{I}) \mid i \notin B\right\}$ to $\left\{B \in \mathcal{S}_{A}(\mathcal{I}) \mid i \in B\right\}$.

Let $\underline{x}_{\mathcal{I}}=\left(x_{I}\right)_{I \in \mathcal{I}} \in \mathbb{R}^{\mathcal{I}}$ and let $B \in \mathcal{S}_{A}(\mathcal{I})$ be such that $i \notin B$. Regrouping the terms corresponding to $B$ and $B \sqcup\{i\}$ in the sum defining $F_{\mathcal{I}}\left(\underline{x}_{\mathcal{I}}\right)$ (see Equation (7.2)), we obtain:

$$
\left(\frac{-1}{\pi}\right)^{|A|-|B|-1}\left(\rho_{\left\{\mathcal{I}_{B \sqcup\{i\}}\right\}}\left(\underline{x}_{\mathcal{I}_{B \sqcup\{i\}}}\right)-\frac{1}{\pi} \rho_{\left\{\mathcal{I}_{B}\right\}}\left(\underline{x}_{\mathcal{I}_{B}}\right)\right) .
$$

Note that we have $\mathcal{I}=\mathcal{I}_{B} \sqcup\{\{a\} \mid a \in A \backslash B\}$ and $\mathcal{I}_{B \sqcup\{i\}}=\mathcal{I}_{B} \sqcup\{\{i\}\}$. Let us now assume that $\underline{x}_{\mathcal{I}} \in \mathbb{R}_{\mathcal{J}, \eta(R)}^{\mathcal{I}}$. Since $\{\{i\}\} \in \mathcal{J}$, for all $I \in \mathcal{I} \backslash\{\{i\}\}$ we have $\left|x_{I}-x_{\{i\}}\right| \geqslant \eta(R)$, and $\mathcal{I}_{\eta(R)}\left(\underline{x}_{\mathcal{I}_{B \sqcup\{i\}}}\right)=\mathcal{J}_{\mathcal{I}_{B \sqcup\{i\}}} \leqslant\left\{\mathcal{I}_{B},\{i\}\right\}$. Then, by Lemma 6.41 and Corollary 6.44, the righthand side of Equation (7.13) is $o\left(R^{-\frac{|A|}{2}}\right)$, uniformly over $\mathbb{R}_{\mathcal{J}, \eta(R)}^{\mathcal{I}}$. Here, we also used the fact that $\rho_{1}$ is constant equal to $\frac{1}{\pi}$ (see Example 3.3) and that $\|\kappa\|_{|A|, \eta(R)}=o\left(R^{-|A|}\right.$ ), since $\eta$ satisfies the conclusion of Lemma 7.6.

Corollary 7.11. In the same setting as Lemma 7.10, let $\left(\phi_{a}\right)_{a \in A}$ be Lebesgue-integrable and essentially bounded functions. Then, as $R \rightarrow+\infty$, we have:

$$
\int_{\mathbb{R}_{\mathcal{J}, \eta(R)}^{\mathcal{I}}} \iota_{\mathcal{I}}^{*} \phi_{R}\left(\underline{x}_{\mathcal{I}}\right) F_{\mathcal{I}}\left(\underline{x}_{\mathcal{I}}\right) \mathrm{d} \underline{x}_{\mathcal{I}}=o\left(R^{\frac{|A|}{2}}\right),
$$

where $\eta:[0,+\infty) \rightarrow[0,+\infty)$ is a function satisfying the conditions of Lemma 7.6.

Proof. Our hypotheses ensure that $\iota_{\mathcal{I}}^{*} \phi$ is integrable on $\mathbb{R}^{\mathcal{I}}$. Then, by Lemma 7.10, we have:

$$
\int_{\mathbb{R}_{\mathcal{J}, \eta(R)}^{\mathcal{I}}} \iota_{\mathcal{I}}^{*} \phi_{R}\left(\underline{x}_{\mathcal{I}}\right) F_{\mathcal{I}}\left(\underline{x}_{\mathcal{I}}\right) \mathrm{d} \underline{x}_{\mathcal{I}}=o\left(R^{-\frac{|A|}{2}}\right) \int_{\mathbb{R}^{\mathcal{I}}}\left|\iota_{\mathcal{I}}^{*} \phi\left(\frac{\underline{x}_{\mathcal{I}}}{R}\right)\right| \mathrm{d} \underline{x}_{\mathcal{I}}=o\left(R^{|\mathcal{I}|-\frac{|A|}{2}}\right) .
$$

Since $\mathcal{I} \in \mathcal{P}_{A}$, we have $|\mathcal{I}| \leqslant|A|$, which concludes the proof.

Example 7.12. Let us consider the case where $A=\{a, b\}$ and $\mathcal{I}=\mathcal{I}_{\min }(A)=\{\{a\},\{b\}\}$. Applying Corollary 7.11 with $\mathcal{J}=\mathcal{I}_{\min }(\mathcal{I})=\{\{\{a\}\},\{\{b\}\}\}$, we have:

$$
\begin{aligned}
\int_{\mathbb{R}_{\mathcal{J}, \eta(R)}^{\mathcal{I}}} \iota_{\mathcal{I}}^{*} \phi_{R}\left(\underline{x}_{\mathcal{I}}\right) F_{\mathcal{I}}\left(\underline{x}_{\mathcal{I}}\right) \mathrm{d} \underline{x}_{\mathcal{I}} & =\int_{\left\{(x, y) \in \mathbb{R}^{2}|| x-y \mid>\eta(R)\right\}} \phi_{a}\left(\frac{x}{R}\right) \phi_{b}\left(\frac{y}{R}\right) F_{\mathcal{I}}(x, y) \mathrm{d} x \mathrm{~d} y \\
& =o(R) .
\end{aligned}
$$

Besides, by Example 7.4, we know that:

$$
\int_{\mathbb{R}^{2}} \phi_{a}\left(\frac{x}{R}\right) \phi_{b}\left(\frac{y}{R}\right) F_{\mathcal{I}}(x, y) \mathrm{d} x \mathrm{~d} y=R\left(\sigma^{2}-\frac{1}{\pi}\right) \int_{\mathbb{R}^{2}} \phi_{a}(x) \phi_{b}(x) \mathrm{d} x+o(R),
$$


where $\sigma^{2}$ is the constant appearing in Proposition 1.11. Since $\mathcal{P}_{\mathcal{I}}=\{\mathcal{J},\{\mathcal{I}\}$, this shows that:

$$
\begin{aligned}
\int_{\mathbb{R}_{\{\mathcal{I}\}, \eta(R)}^{\mathcal{I}}} \iota_{\mathcal{I}}^{*} \phi_{R}\left(\underline{x}_{\mathcal{I}}\right) F_{\mathcal{I}}\left(\underline{x}_{\mathcal{I}}\right) \mathrm{d} \underline{x}_{\mathcal{I}} & =\int_{\left\{(x, y) \in \mathbb{R}^{2}|| x-y \mid \leqslant \eta(R)\right\}} \phi_{a}\left(\frac{x}{R}\right) \phi_{b}\left(\frac{y}{R}\right) F_{\mathcal{I}}(x, y) \mathrm{d} x \mathrm{~d} y \\
& =R\left(\sigma^{2}-\frac{1}{\pi}\right) \int_{\mathbb{R}^{2}} \phi_{a}(x) \phi_{b}(x) \mathrm{d} x+o(R) .
\end{aligned}
$$

\subsection{Contribution of the partitions into pairs}

The goal of this section is to study the behavior of the function $F_{\mathcal{I}}$, introduced in Lemma 7.3, on some particular pieces of the decomposition $\mathbb{R}^{\mathcal{I}}=\bigsqcup_{\mathcal{J} \in \mathcal{P}_{\mathcal{I}}} \mathbb{R}_{\mathcal{J}, \eta(R)}^{\mathcal{I}}$. More precisely, we will consider pieces indexed by partitions into pairs.

Definition 7.13. Let $A \neq \emptyset$ be a finite set and $\mathcal{I} \in \mathcal{P}_{A}$, we denote by $\mathcal{I}^{\prime}=\{I \in \mathcal{I}|| I \mid \geqslant 2\}$ and by $\mathcal{I}^{\prime \prime}=\{I \in \mathcal{I}|| I \mid=1\}$. We denote by $\mathfrak{C}_{A}$ the set of couples $(\mathcal{I}, \mathcal{J})$ such that $\mathcal{I} \in \mathcal{P}_{A}$, $\mathcal{J} \in \mathcal{P}_{\mathcal{I}}$ and the following two conditions are satisfied:

1. for all $I \in \mathcal{I}^{\prime}$, we have $|I|=2$;

2. there exists $\mathcal{J}^{\prime} \in \mathcal{P P}_{\mathcal{I}^{\prime \prime}}$ such that $\mathcal{J}=\mathcal{J}^{\prime} \sqcup \mathcal{J}^{\prime \prime}$, where $\mathcal{J}^{\prime \prime}=\left\{\{I\} \mid I \in \mathcal{I}^{\prime}\right\}$.

As usual, if $A=\{1, \ldots, p\}$, we denote by $\mathfrak{C}_{p}=\mathfrak{C}_{A}$.

Remark 7.14. If $(\mathcal{I}, \mathcal{J}) \in \mathfrak{C}_{A}$, then $\mathcal{I}^{\prime \prime}$ admits a partition into pairs, hence $\left|\mathcal{I}^{\prime \prime}\right|$ is even. Moreover, $|A|=2\left|\mathcal{I}^{\prime}\right|+\left|\mathcal{I}^{\prime \prime}\right|$ is also even.

Lemma 7.15. Let $A$ be a non-empty finite set and let us assume that $f$ is a $\mathcal{C}^{|A|}$-process such that $\|\kappa\|_{|A|, \eta}=o\left(\eta^{-4|A|}\right)$ at infinity. Let $\eta:[0,+\infty) \rightarrow[0,+\infty)$ be a function satisfying the conditions of Lemma 7.6 with $p=|A|$.

Let $(\mathcal{I}, \mathcal{J}) \in \mathfrak{C}_{A}$ and let $\mathcal{I}^{\prime}, \mathcal{I}^{\prime \prime}, \mathcal{J}^{\prime}$ and $\mathcal{J}^{\prime \prime}$ be as in Definition 7.13. Then the following holds uniformly for all $\underline{x}_{\mathcal{I}} \in \mathbb{R}_{\mathcal{J}, \eta(R)}^{\mathcal{I}}$ :

$$
F_{\mathcal{I}}\left(\underline{x}_{\mathcal{I}}\right)=\left(\frac{1}{\pi}\right)^{\left|\mathcal{I}^{\prime}\right|} \prod_{J \in \mathcal{J}^{\prime}} F_{J}\left(\underline{x}_{J}\right)+o\left(R^{-\frac{|A|}{2}}\right) .
$$

Proof. Let us denote by $S=\bigsqcup_{I \in \mathcal{I}^{\prime \prime}} I$. We have $|S|=\left|\mathcal{I}^{\prime \prime}\right|$, and by Remark 7.14 this cardinality is even. By Lemma 7.8, we have the following uniform estimate: for all $\underline{x}_{\mathcal{I}} \in \mathbb{R}_{\mathcal{J}, \eta(R)}^{\mathcal{I}}$,

$$
F_{\mathcal{I}}\left(\underline{x}_{\mathcal{I}}\right)=F_{\mathcal{I}^{\prime}}\left(\underline{x}_{\mathcal{I}^{\prime}}\right) \prod_{J \in \mathcal{J}^{\prime}} F_{J}\left(\underline{x}_{J}\right)+o\left(R^{-\frac{|A|}{2}}\right) .
$$

Note that we have $\mathcal{I}^{\prime} \in \mathcal{P}_{A \backslash S}$. Recalling Definition 7.1, we have $\mathcal{S}_{A \backslash S}\left(\mathcal{I}^{\prime}\right)=\{A \backslash S\}$ because of Condition 1 in Definition 7.13. Hence we obtain $F_{\mathcal{I}^{\prime}}=\rho_{\left\{\mathcal{I}^{\prime}\right\}}$ by Equation (7.2). By Condition 2 in Definition 7.13 we have $\mathcal{J}_{\mathcal{I}^{\prime}}=\mathcal{J}^{\prime \prime}$. Then, for any $\underline{x}_{\mathcal{I}} \in \mathbb{R}_{\mathcal{J}, \eta(R)}^{\mathcal{I}}$ we have $\underline{x}_{\mathcal{I}^{\prime}} \in \mathbb{R}_{\mathcal{J}^{\prime \prime}, \eta(R)}^{\mathcal{I}^{\prime}}$, and by Proposition 6.43 , we obtain:

$$
F_{\mathcal{I}^{\prime}}\left(\underline{x}_{\mathcal{I}^{\prime}}\right)=\rho_{\left\{\mathcal{I}^{\prime}\right\}}\left(\underline{x}_{\mathcal{I}^{\prime}}\right)=\left(\prod_{J \in \mathcal{J}^{\prime \prime}} \rho_{\{J\}}\left(\underline{x}_{J}\right)\right)\left(1+O\left(\|\kappa\|_{|A|, \eta(R)}\right)^{\frac{1}{2}}\right),
$$

uniformly for $\underline{x}_{\mathcal{I}} \in \mathbb{R}_{\mathcal{J}, \eta(R)}^{\mathcal{I}}$. Our hypotheses on $\kappa$ and $\eta$ ensure that $\|\kappa\|_{|A|, \eta(R)}=o\left(R^{-\frac{|A|}{2}}\right)$. Moreover, for all $J \in \mathcal{J}^{\prime \prime}$ we have $|J|=1$, so that $\rho_{\{J\}}=\rho_{1}$ is constant equal to $\frac{1}{\pi}$, see Example 3.3. The conclusion follows from the boundedness of the functions $F_{J}$ with $J \in \mathcal{J}^{\prime}$ (see Lemma 7.5) and from $\left|\mathcal{J}^{\prime \prime}\right|=\left|\mathcal{I}^{\prime}\right|$. 


\subsection{Conclusion of the proof}

In this section, we finally conclude the proof of Theorem 1.6. In all this section, we fix an integer $p \geqslant 2$. We consider a normalized centered stationary Gaussian process $f$ of class $\mathcal{C}^{p}$. The correlation function $\kappa$ of $f$ is such that $\|\kappa\|_{p, \eta}=o\left(\eta^{-4 p}\right)$ as $\eta \rightarrow+\infty$. We fix a function $\eta:[0,+\infty) \rightarrow[0,+\infty)$ such that as $R \rightarrow+\infty$ we have: $\eta(R) \rightarrow+\infty$, $\eta(R)=o\left(R^{\frac{1}{4}}\right)$, and $\|\kappa\|_{p, \eta(R)}=o\left(R^{-p}\right)$. The existence of such a function was proved in Lemma 7.6. Finally, we consider test-functions $\phi_{1}, \ldots, \phi_{p}$ is the sense of Definition 1.2.

Lemma 7.16 (Error terms). Let $\mathcal{I} \in \mathcal{P}_{p}$ and $\mathcal{J} \in \mathcal{P}_{\mathcal{I}}$ be such that $(\mathcal{I}, \mathcal{J}) \notin \mathfrak{C}_{p}$, where $\mathfrak{C}_{p}$ is defined by Definition 7.13. Then, as $R \rightarrow+\infty$, we have:

$$
\int_{\mathbb{R}_{\mathcal{J}, \eta(R)}^{\mathcal{I}}} \iota_{\mathcal{I}}^{*} \phi_{R}\left(\underline{x}_{\mathcal{I}}\right) F_{\mathcal{I}}\left(\underline{x}_{\mathcal{I}}\right) \mathrm{d} \underline{x}_{\mathcal{I}}=o\left(R^{\frac{p}{2}}\right) .
$$

Proof. As in Definition 7.13, let us set $\mathcal{I}^{\prime}=\{I \in \mathcal{I}|| I \mid \geqslant 2\}$ and $\mathcal{I}^{\prime \prime}=\{I \in \mathcal{I}|| I \mid=1\}$. Since $\mathcal{I}=\mathcal{I}^{\prime} \sqcup \mathcal{I}^{\prime \prime} \in \mathcal{P}_{p}$, we have:

$$
2\left|\mathcal{I}^{\prime}\right|+\left|\mathcal{I}^{\prime \prime}\right| \leqslant p
$$

and equality holds if and only if $|I|=2$ for all $I \in \mathcal{I}^{\prime}$, that is if and only if Condition 1 of Definition 7.13 is satisfied.

Let $J \in \mathcal{J}$ be such that $|J|=1$. There exists $I \in \mathcal{I}$ such that $J=\{I\}$. If $I \in \mathcal{I}^{\prime \prime}$, that is if $I$ is a singleton, then we are in the situation studied in Section 7.3. In this case, Equation (7.14) holds by Corollary 7.11.

In the following, we assume that we are not in the previous situation. That is, for all $J \in \mathcal{J}$ such that $|J|=1$, we have $J \subset \mathcal{I}^{\prime}$. Let us prove that Equation (7.14) holds in this case. We denote by $\mathcal{J}^{\prime}=\left\{J \in \mathcal{J}\left|J \subset \mathcal{I}^{\prime \prime},\right| J \mid=2\right\}$ and by $\mathcal{J}^{\prime \prime}=\mathcal{J} \backslash \mathcal{J}^{\prime}$. Finally, let $S=\bigsqcup_{J \in \mathcal{J}^{\prime}} \bigsqcup_{I \in J} I \subset\{1, \ldots, p\}$. By definition of $S$ we have $\mathcal{I}_{S}=\{\{s\} \mid s \in S\} \subset \mathcal{I}^{\prime \prime} \subset \mathcal{I}$ and $\mathcal{J}^{\prime} \in \mathcal{P P}_{\mathcal{I}_{S}}$. We also have $\mathcal{J}^{\prime \prime} \in \mathcal{P}_{\mathcal{I} \backslash \mathcal{I}_{S}}$ and $\mathcal{J}=\mathcal{J}^{\prime} \sqcup \mathcal{J}^{\prime \prime}$. By Lemma 7.9, the left-hand side of Equation (7.14) equals $O\left(R^{|\mathcal{J}|} \eta(R)^{|\mathcal{I}|-2\left|\mathcal{J}^{\prime}\right|-\left|\mathcal{J}^{\prime \prime}\right|}\right)$. Since $\eta(R)=o\left(R^{\frac{1}{4}}\right)$, there exists a function $\alpha$ such that $\alpha(R) \underset{R \rightarrow+\infty}{\longrightarrow} 0$ and $\eta(R)=\alpha(R) R^{\frac{1}{4}}$. Then, left-hand side of (7.14) is:

$$
O\left(R^{|\mathcal{J}|+\frac{|\mathcal{I}|-2\left|\mathcal{J}^{\prime}\right|-\left|\mathcal{J}^{\prime \prime}\right|}{4}} \alpha(R)^{|\mathcal{I}|-2\left|\mathcal{J}^{\prime}\right|-\left|\mathcal{J}^{\prime \prime}\right|}\right) .
$$

Let $J \in \mathcal{J}^{\prime \prime}$. Since $\mathcal{J}^{\prime \prime}$ is a partition of $\mathcal{I} \backslash \mathcal{I}_{S}=\mathcal{I}^{\prime} \sqcup\left(\mathcal{I}^{\prime \prime} \backslash \mathcal{I}_{S}\right)$, if $J \cap \mathcal{I}^{\prime}=\emptyset$ then $J \subset \mathcal{I}^{\prime \prime} \backslash \mathcal{I}_{S}$. In this case, we assumed that $|J| \neq 1$, and moreover $|J| \neq 2$ by definition of $\mathcal{J}^{\prime}$ and $\mathcal{J}^{\prime \prime}$. Thus, either there exists $I \in \mathcal{I}^{\prime} \cap J$, or $J \subset \mathcal{I}^{\prime \prime} \backslash \mathcal{I}_{S}$ and $|J| \geqslant 3$. This proves that:

$$
\left|\mathcal{J}^{\prime \prime}\right| \leqslant\left|\mathcal{I}^{\prime}\right|+\frac{1}{3}\left(\left|\mathcal{I}^{\prime \prime}\right|-\left|\mathcal{I}_{S}\right|\right)
$$

Since $\left|\mathcal{I}_{S}\right|=2\left|\mathcal{J}^{\prime}\right|$, we have $2\left|\mathcal{J}^{\prime}\right|+3\left|\mathcal{J}^{\prime \prime}\right| \leqslant 3\left|\mathcal{I}^{\prime}\right|+\left|\mathcal{I}^{\prime \prime}\right|$. Hence,

$$
|\mathcal{J}|+\frac{|\mathcal{I}|-2\left|\mathcal{J}^{\prime}\right|-\left|\mathcal{J}^{\prime \prime}\right|}{4}=\frac{1}{4}\left(2\left|\mathcal{J}^{\prime}\right|+3\left|\mathcal{J}^{\prime \prime}\right|+\left|\mathcal{I}^{\prime}\right|+\left|\mathcal{I}^{\prime \prime}\right|\right) \leqslant \frac{1}{2}\left(2\left|\mathcal{I}^{\prime}\right|+\left|\mathcal{I}^{\prime \prime}\right|\right) .
$$

Then, by Equation (7.15), we have:

$$
|\mathcal{J}|+\frac{|\mathcal{I}|-2\left|\mathcal{J}^{\prime}\right|-\left|\mathcal{J}^{\prime \prime}\right|}{4} \leqslant \frac{p}{2} .
$$

If this inequality is strict, then (7.16) equals a $o\left(R^{\frac{p}{2}}\right)$, which proves that Equation (7.14) holds.

If equality holds in Equation (7.17), then it must hold in Equation (7.15), which implies that $(\mathcal{I}, \mathcal{J})$ satisfies Condition 1 in Definition 7.13. In this case, (7.16) is a 
$O\left(R^{\frac{p}{2}} \alpha(R)^{|\mathcal{I}|-2\left|\mathcal{J}^{\prime}\right|-\left|\mathcal{J}^{\prime \prime}\right|}\right)$ and it is enough to check that $2\left|\mathcal{J}^{\prime}\right|+\left|\mathcal{J}^{\prime \prime}\right|<|\mathcal{I}|$, because $\alpha(R)=o(1)$. Since $\mathcal{J}=\mathcal{J}^{\prime} \sqcup \mathcal{J}^{\prime \prime} \in \mathcal{P}_{\mathcal{I}}$ and $|J|=2$ for all $J \in \mathcal{J}^{\prime}$, we have:

$$
2\left|\mathcal{J}^{\prime}\right|+\left|\mathcal{J}^{\prime \prime}\right| \leqslant|\mathcal{I}|
$$

and equality holds if and only if $|J|=1$ for all $J \in \mathcal{J}^{\prime \prime}$. If equality held in Equation (7.18) then, under our assumptions, we would have $|J|=1$ and $J \subset \mathcal{I}^{\prime}$ for all $J \in \mathcal{J}^{\prime \prime}$, hence $\mathcal{J}^{\prime \prime} \subset \mathcal{I}_{\min }\left(\mathcal{I}^{\prime}\right)$. Since $\mathcal{J}^{\prime} \in \mathcal{P}_{\mathcal{I}_{S}}$ and $\mathcal{I}_{S} \subset \mathcal{I}^{\prime \prime}=\mathcal{I} \backslash \mathcal{I}^{\prime}$, the only possibility for this to happen is that $\mathcal{J}^{\prime \prime}=\mathcal{I}_{\text {min }}\left(\mathcal{I}^{\prime}\right)$ and $\mathcal{I}_{S}=\mathcal{I}^{\prime \prime}$. Thus, if equality held in Equation (7.18) then $(\mathcal{I}, \mathcal{J})$ would satisfy Condition 2 of Definition 7.13. Since we already assumed that $(\mathcal{I}, \mathcal{J})$ satisfies Condition 1 , this would imply $(\mathcal{I}, \mathcal{J}) \in \mathfrak{C}_{p}$, which is a contradiction. Finally, the inequality is strict in Equation (7.18). Hence, (7.16) is a $o\left(R^{\frac{p}{2}}\right)$, which concludes the proof.

Lemma 7.17 (Leading terms). Let $\mathcal{I} \in \mathcal{P}_{p}$ and $\mathcal{J} \in \mathcal{P}_{\mathcal{I}}$ be such that $(\mathcal{I}, \mathcal{J}) \in \mathfrak{C}_{p}$. Let $\mathcal{I}^{\prime}, \mathcal{I}^{\prime \prime}, \mathcal{J}^{\prime}$ and $\mathcal{J}^{\prime \prime}$ be as in Definition 7.13. Then, as $R \rightarrow+\infty$, we have:

$$
\begin{aligned}
\int_{\underline{x}_{\mathcal{I}} \in \mathbb{R}_{\mathcal{J}, \eta(R)}^{\mathcal{I}}} \iota_{\mathcal{I}}^{*} \phi_{R}\left(\underline{x}_{\mathcal{I}}\right) F_{\mathcal{I}}\left(\underline{x}_{\mathcal{I}}\right) \mathrm{d} \underline{x}_{\mathcal{I}}= \\
\quad\left(\prod_{\{i, j\} \in \mathcal{I}^{\prime}} \frac{R}{\pi} \int_{\mathbb{R}} \phi_{i}(x) \phi_{j}(x) \mathrm{d} x\right)\left(\prod_{J \in \mathcal{J}^{\prime}} \int_{\mathbb{R}^{J}} \phi_{J}\left(\frac{\underline{x}_{J}}{R}\right) F_{J}\left(\underline{x}_{J}\right) \mathrm{d} \underline{x}_{J}\right)+o\left(R^{\frac{p}{2}}\right) .
\end{aligned}
$$

Proof. For any point $\underline{x}_{\mathcal{I}} \in \mathbb{R}^{\mathcal{I}}$, we have:

$$
\iota_{\mathcal{I}}^{*} \phi_{R}\left(\underline{x}_{\mathcal{I}}\right)=\prod_{I \in \mathcal{I}} \prod_{i \in I} \phi_{i}\left(\frac{x_{I}}{R}\right)=\left(\prod_{\{i, j\}=I \in \mathcal{I}^{\prime}} \phi_{i}\left(\frac{x_{I}}{R}\right) \phi_{j}\left(\frac{x_{I}}{R}\right)\right)\left(\prod_{\{i\} \in \mathcal{I}^{\prime \prime}} \phi_{i}\left(\frac{x_{\{i\}}}{R}\right)\right) .
$$

Note that, for all $J=\{\{i\},\{j\}\} \in \mathcal{J}^{\prime}$ we have $\phi_{J}=\phi_{\{i\}} \otimes \phi_{\{j\}}=\phi_{i} \otimes \phi_{j}$ (see Notation 2.1). Hence, by Lemma 7.15, for all $\underline{x}_{I} \in \mathbb{R}_{\mathcal{J}, \eta(R)}^{\mathcal{I}}$, we have that $\iota_{\mathcal{I}}^{*} \phi_{R}\left(\underline{x}_{\mathcal{I}}\right) F_{\mathcal{I}}\left(\underline{x}_{\mathcal{I}}\right)$ equals:

$$
\left(\prod_{\{i, j\}=I \in \mathcal{I}^{\prime}} \frac{1}{\pi} \phi_{i}\left(\frac{x_{I}}{R}\right) \phi_{j}\left(\frac{x_{I}}{R}\right)\right)\left(\prod_{J \in \mathcal{J}^{\prime}} \phi_{J}\left(\frac{\underline{x}_{J}}{R}\right) F_{J}\left(\underline{x}_{J}\right)\right)+\iota_{\mathcal{I}}^{*} \phi_{R}\left(\underline{x}_{\mathcal{I}}\right) o\left(R^{-\frac{p}{2}}\right),
$$

and we want to compute the integral of (7.19) over $\mathbb{R}_{\mathcal{J}, \eta(R)}^{\mathcal{I}}$. Our assumptions on the the test-functions $\phi_{1}, \ldots, \phi_{p}$ ensure that $\iota_{\mathcal{I}}^{*} \phi$ is integrable on $\mathbb{R}^{\mathcal{I}}$. Hence, since $|\mathcal{I}| \leqslant p$, the contribution to the integral of the error term in Equation (7.19) is:

$$
\int_{\mathbb{R}_{\mathcal{J}, \eta(R)}^{\mathcal{I}}} \iota_{\mathcal{I}}^{*} \phi_{R}\left(\underline{x}_{\mathcal{I}}\right) o\left(R^{-\frac{p}{2}}\right) \mathrm{d} \underline{x}_{\mathcal{I}}=o\left(R^{-\frac{p}{2}}\right) \int_{\mathbb{R}_{\mathcal{J}, \eta(R)}^{\mathcal{I}}}\left|\iota_{\mathcal{I}}^{*} \phi_{R}\left(\underline{x}_{\mathcal{I}}\right)\right| \mathrm{d} \underline{x}_{\mathcal{I}}=o\left(R^{|\mathcal{I}|-\frac{p}{2}}\right)=o\left(R^{\frac{p}{2}}\right) .
$$

Let us check that:

$$
\mathbb{R}_{\mathcal{J}, \eta(R)}^{\mathcal{I}} \subset\left(\prod_{I \in \mathcal{I}^{\prime}} \mathbb{R}\right) \times\left(\prod_{J \in \mathcal{J}^{\prime}} \mathbb{R}_{\{J\}, \eta(R)}^{J}\right) \subset \bigsqcup_{\mathcal{K} \geqslant \mathcal{J}} \mathbb{R}_{\mathcal{K}, \eta(R)}^{\mathcal{I}} .
$$

In order to prove the first inclusion, let $\underline{x}_{\mathcal{I}} \in \mathbb{R}_{\mathcal{J}, \eta(R)}^{\mathcal{I}}$. Since $\mathcal{J}$ satisfies Condition 2 of Definition 7.13, the associated graph $G_{\eta(R)}\left(\underline{x}_{\mathcal{I}}\right)$ (see Definition 6.1) is formed of $\left|\mathcal{I}^{\prime}\right|$ isolated vertices $\left\{I \mid I \in \mathcal{I}^{\prime}\right\}$ and $\left|\mathcal{J}^{\prime}\right|$ pairs of vertices of the form $\left\{I, I^{\prime}\right\} \in \mathcal{J}^{\prime}$ with an edge between $I$ and $I^{\prime}$. Hence, for all $J=\left\{I, I^{\prime}\right\} \in \mathcal{J}^{\prime}$, we have $\left|x_{I}-x_{I^{\prime}}\right| \leqslant \eta(R)$, and $\underline{x}_{J} \in \mathbb{R}_{\{J\}, \eta(R)}^{J}$. Let us now prove the second inclusion in Equation (7.20). Let $\underline{x}_{\mathcal{I}}=\left(x_{I}\right)_{I \in \mathcal{I}} \in \mathbb{R}^{\mathcal{I}}$ and let us assume that $\underline{x}_{\mathcal{I}}$ belongs to the middle set in Equation (7.20). 
By Definition 6.1, for all $J=\left\{I, I^{\prime}\right\} \in \mathcal{J}^{\prime}$, the graph $G_{\eta(R)}\left(\underline{x}_{\mathcal{I}}\right)$ associated with $\underline{x}_{\mathcal{I}}$ has an edge between $I$ and $I^{\prime}$. Hence the associated partition $\mathcal{K}=\mathcal{I}_{\eta(R)}\left(\underline{x}_{\mathcal{I}}\right)$ (see Definition 6.2) is such that, for all $J \in \mathcal{J}^{\prime}$ there exists $K \in \mathcal{K}$ such that $J \subset K$. Since $\mathcal{J}$ satisfies Condition 2 in Definition 7.13, this is enough to ensure that $\mathcal{K} \geqslant \mathcal{J}$. Note that, in general, both inclusions in Equation (7.20) are strict.

Let $\mathcal{K} \in \mathcal{P}_{\mathcal{I}}$ be such that $\mathcal{K}>\mathcal{J}$. This ensures that $(\mathcal{I}, \mathcal{K}) \notin \mathfrak{C}_{p}$.Then, by Lemma 7.16, the integral of the leading term in Equation (7.19) over

$$
\left(\left(\prod_{I \in \mathcal{I}^{\prime}} \mathbb{R}\right) \times\left(\prod_{J \in \mathcal{J}^{\prime \prime}} \mathbb{R}_{\{J\}, \eta(R)}^{J}\right)\right) \cap \mathbb{R}_{\mathcal{K}, \eta(R)}^{\mathcal{I}}
$$

equals $o\left(R^{\frac{p}{2}}\right)$. This proves that:

$$
\begin{aligned}
& \int_{\underline{x}_{\mathcal{I}} \in \mathbb{R}_{\mathcal{J}, \eta(R)}^{\mathcal{I}}} \iota_{\mathcal{I}}^{*} \phi_{R}\left(\underline{x}_{\mathcal{I}}\right) F_{\mathcal{I}}\left(\underline{x}_{\mathcal{I}}\right) \mathrm{d} \underline{x}_{\mathcal{I}}= \\
& \left(\prod_{\{i, j\} \in \mathcal{I}^{\prime}} \frac{R}{\pi} \int_{\mathbb{R}} \phi_{i}(x) \phi_{j}(x) \mathrm{d} x\right)\left(\prod_{J \in \mathcal{J}^{\prime}} \int_{\mathbb{R}_{\{J\}, \eta(R)}^{J}} \phi_{J}\left(\frac{\underline{x}_{J}}{R}\right) F_{J}\left(\underline{x}_{J}\right) \mathrm{d} \underline{x}_{J}\right)+o\left(R^{\frac{p}{2}}\right) .
\end{aligned}
$$

In order to conclude the proof, we need to replace the integral over $\mathbb{R}_{\{J\}, \eta(R)}^{J}$ by an integral over $\mathbb{R}^{J}$ in Equation (7.21), for all $J \in \mathcal{J}^{\prime}$. Let $J=\left\{I, I^{\prime}\right\} \in \mathcal{J}^{\prime}$, we have:

$$
\begin{aligned}
\int_{\mathbb{R}_{\{J\}, \eta(R)}^{J}} \phi_{J}\left(\frac{\underline{x}_{J}}{R}\right) & F_{J}\left(\underline{x}_{J}\right) \mathrm{d} \underline{x}_{J} \\
& =\int_{\mathbb{R}^{J}} \phi_{J}\left(\frac{\underline{x}_{J}}{R}\right) F_{J}\left(\underline{x}_{J}\right) \mathrm{d} \underline{x}_{J}-\int_{\mathbb{R}_{\left\{\{I\},\left\{I^{\prime}\right\}\right\}, \eta(R)}^{J}} \phi_{J}\left(\frac{\underline{x}_{J}}{R}\right) F_{J}\left(\underline{x}_{J}\right) \mathrm{d} \underline{x}_{J} \\
& =\int_{\mathbb{R}^{J}} \phi_{J}\left(\frac{\underline{x}_{J}}{R}\right) F_{J}\left(\underline{x}_{J}\right) \mathrm{d} \underline{x}_{J}+o(R),
\end{aligned}
$$

by Corollary 7.11. Moreover, as in Example 7.12, we have:

$$
\int_{\mathbb{R}^{J}} \phi_{J}\left(\frac{\underline{x}_{J}}{R}\right) F_{J}\left(\underline{x}_{J}\right) \mathrm{d} \underline{x}_{J}=O(R) .
$$

Since $\left|\mathcal{I}^{\prime}\right|+\left|\mathcal{J}^{\prime}\right|=\left|\mathcal{I}^{\prime}\right|+\frac{1}{2}\left|\mathcal{I}^{\prime \prime}\right|=\frac{p}{2}$, this yields the claimed estimate.

Proof of Theorem 1.6. By Lemma 7.3 and the fact that $\mathbb{R}^{\mathcal{I}}=\bigsqcup_{\mathcal{J} \in \mathcal{P}_{\mathcal{I}}} \mathbb{R}_{\mathcal{J}, \eta(R)}^{\mathcal{I}}$ for all $\mathcal{I} \in \mathcal{P}_{p}$, we have:

$$
m_{p}\left(\nu_{R}\right)\left(\phi_{1}, \ldots, \phi_{p}\right)=\sum_{\mathcal{I} \in \mathcal{P}_{p}} \sum_{\mathcal{J} \in \mathcal{P}_{\mathcal{I}}} \int_{\underline{x}_{\mathcal{I}} \in \mathbb{R}_{\mathcal{J}, \eta(R)}^{\mathcal{I}}}\left(\iota_{\mathcal{I}}^{*} \phi_{R}\right)\left(\underline{x}_{\mathcal{I}}\right) F_{\mathcal{I}}\left(\underline{x}_{\mathcal{I}}\right) \mathrm{d} \underline{x}_{\mathcal{I}} .
$$

By Lemma 7.16, up to an error term of the form $o\left(R^{\frac{p}{2}}\right)$ we need only consider the terms in this double sum indexed by $(\mathcal{I}, \mathcal{J}) \in \mathfrak{C}_{p}$, where $\mathfrak{C}_{p}$ is defined by Definition 7.13. The expression of these terms is given by Lemma 7.17. Thus, we have:

$$
\begin{aligned}
& m_{p}\left(\nu_{R}\right)\left(\phi_{1}, \ldots, \phi_{p}\right)= \\
& \quad \sum_{(\mathcal{I}, \mathcal{J}) \in \mathfrak{C}_{p}}\left(\prod_{\{i, j\} \in \mathcal{I}^{\prime}} \frac{R}{\pi} \int_{\mathbb{R}} \phi_{i}(x) \phi_{j}(x) \mathrm{d} x\right)\left(\prod_{J \in \mathcal{J}^{\prime}} \int_{\mathbb{R}^{J}} \phi_{J}\left(\frac{\underline{x}_{J}}{R}\right) F_{J}\left(\underline{x}_{J}\right) \mathrm{d} \underline{x}_{J}\right)+o\left(R^{\frac{p}{2}}\right),
\end{aligned}
$$

where we used the same notations as in Definition 7.13. Recall that $\mathcal{I}^{\prime}=\{I \in \mathcal{I}|| I \mid=2\}$ and $\mathcal{I}^{\prime \prime}=\{I \in \mathcal{I}|| I \mid=1\}$, so that $\mathcal{I}=\mathcal{I}^{\prime} \sqcup \mathcal{I}^{\prime \prime}$ thanks to Condition 1 in Definition 7.13. 
Recall also that $\mathcal{J}^{\prime \prime}=\left\{\{I\} \mid I \in \mathcal{I}^{\prime}\right\}$ and $\mathcal{J}=\mathcal{J}^{\prime} \sqcup \mathcal{J}^{\prime \prime}$ for some $\mathcal{J}^{\prime} \in \mathcal{P P}_{\mathcal{I}^{\prime \prime}}$, thanks to Condition 2 in Definition 7.13.

We just wrote $m_{p}\left(\nu_{R}\right)\left(\phi_{1}, \ldots, \phi_{p}\right)$ as a sum of terms indexed by $\mathfrak{C}_{p}$. In the following, we define a bijection $\Phi: \mathfrak{C}_{p} \rightarrow\left\{(\Pi, \mathcal{S}) \mid \Pi \in \mathcal{P P} P_{p}, \mathcal{S} \subset \Pi\right\}$, which will allow us to rewrite $m_{p}\left(\nu_{R}\right)\left(\phi_{1}, \ldots, \phi_{p}\right)$ as sum over $\left\{(\Pi, \mathcal{S}) \mid \Pi \in \mathcal{P P}{ }_{p}, \mathcal{S} \subset \Pi\right\}$, by a change of variable.

- Let $(\mathcal{I}, \mathcal{J}) \in \mathfrak{C}_{p}$, we denote by $S=\left\{i \mid\{i\} \in \mathcal{I}^{\prime \prime}\right\}$ and by $\mathcal{S}=\left\{\{i, j\} \mid\{\{i\},\{j\}\} \in \mathcal{J}^{\prime}\right\}$. Since $\mathcal{J}^{\prime} \in \mathcal{P P}_{\mathcal{I}^{\prime \prime}}$, we have $\mathcal{S} \in \mathcal{P} P_{S}$. Since $\mathcal{I}^{\prime}$ is a partition of $\bigsqcup_{I \in \mathcal{I}^{\prime}} I=\{1, \ldots, p\} \backslash S$ into pairs, we define a partition into pairs $\Pi \in \mathcal{P P}_{p}$ by $\Pi=\mathcal{I}^{\prime} \sqcup \mathcal{S}$. We obtain a couple $(\Pi, \mathcal{S})$ where $\Pi \in \mathcal{P P} p_{p}$ and $\mathcal{S} \subset \Pi$. Let us denote this couple by $\Phi(\mathcal{I}, \mathcal{J})$.

- Conversely, let $\Pi \in \mathcal{P P} p$ and let $\mathcal{S} \subset \Pi$. We set $S=\bigsqcup_{I \in \mathcal{S}} I$ and $\mathcal{I}^{\prime}=\Pi \backslash \mathcal{S}$, so that $\mathcal{I}^{\prime}$ is a partition into pairs of $\{1, \ldots, p\} \backslash S$. Let us denote by $\mathcal{I}^{\prime \prime}=\{\{i\} \mid i \in S\}$ and by $\mathcal{J}^{\prime}=\{\{\{i\},\{j\}\} \mid\{i, j\} \in \mathcal{S}\}$, so that $\mathcal{J}^{\prime} \in \mathcal{P P}_{\mathcal{I}^{\prime \prime}}$. Finally, let $\mathcal{I}=\mathcal{I}^{\prime} \sqcup \mathcal{I}^{\prime \prime} \in \mathcal{P}_{p}$, and let $\mathcal{J}^{\prime \prime}=\left\{\{I\} \mid I \in \mathcal{I}^{\prime}\right\}$ so that $\mathcal{J}=\mathcal{J}^{\prime} \sqcup \mathcal{J}^{\prime \prime} \in \mathcal{P}_{\mathcal{I}}$. We just defined a couple $(\mathcal{I}, \mathcal{J}) \in \mathfrak{C}_{p}$ that we denote by $\Psi(\Pi, \mathcal{S})$.

By construction, $\Phi$ is a bijection from $\mathfrak{C}_{p}$ to $\{(\Pi, \mathcal{S}) \mid \Pi \in \mathcal{P P}, \mathcal{S} \subset \Pi\}$ such that $\Psi=\Phi^{-1}$. Moreover, for all $(\mathcal{I}, \mathcal{J}) \in \mathfrak{C}_{p}$, denoting by $(\Pi, \mathcal{S})=\Phi(\mathcal{I}, \mathcal{J})$, we have:

$$
\begin{aligned}
& \left(\prod_{\{i, j\} \in \mathcal{I}^{\prime}} \frac{R}{\pi} \int_{\mathbb{R}} \phi_{i}(x) \phi_{j}(x) \mathrm{d} x\right)\left(\prod_{J \in \mathcal{J}^{\prime}} \int_{\mathbb{R}^{J}} \phi_{J}\left(\frac{\underline{x}_{J}}{R}\right) F_{J}\left(\underline{x}_{J}\right) \mathrm{d} \underline{x}_{J}\right)= \\
& \left(\prod_{\{i, j\} \in \Pi \backslash \mathcal{S}} \frac{R}{\pi} \int_{\mathbb{R}} \phi_{i}(x) \phi_{j}(x) \mathrm{d} x\right)\left(\prod_{\{i, j\} \in \mathcal{S}} \int_{\mathbb{R}^{2}} \phi_{i}\left(\frac{x}{R}\right) \phi_{j}\left(\frac{y}{R}\right) F(y-x) \mathrm{d} x \mathrm{~d} y\right),
\end{aligned}
$$

where we used Example 7.4. Hence,

$$
\begin{aligned}
& m_{p}\left(\nu_{R}\right)\left(\phi_{1}, \ldots, \phi_{p}\right)= \\
& \sum_{\Pi \in \mathcal{P} \mathcal{P}_{p}} \sum_{\mathcal{S} \subset \Pi}\left(\prod_{\{i, j\} \in \Pi \backslash \mathcal{S}} \frac{R}{\pi} \int_{\mathbb{R}} \phi_{i}(x) \phi_{j}(x) \mathrm{d} x\right)\left(\prod_{\{i, j\} \in \mathcal{S}} \int_{\mathbb{R}^{2}} \phi_{i}\left(\frac{x}{R}\right) \phi_{j}\left(\frac{y}{R}\right) F(y-x) \mathrm{d} x \mathrm{~d} y\right) \\
& +o\left(R^{\frac{p}{2}}\right) .
\end{aligned}
$$

The leading term on the right-hand side of Equation (7.22) can be rewritten as:

$$
\sum_{\Pi \in \mathcal{P} P_{p}} \prod_{\{i, j\} \in \Pi}\left(\int_{\mathbb{R}^{2}} \phi_{i}\left(\frac{x}{R}\right) \phi_{j}\left(\frac{y}{R}\right) F(y-x) \mathrm{d} x \mathrm{~d} y+\frac{R}{\pi} \int_{\mathbb{R}} \phi_{i}(x) \phi_{j}(x) \mathrm{d} x\right) .
$$

Using the expression of $m_{2}\left(\nu_{R}\right)\left(\phi_{i}, \phi_{j}\right)$ derived in Lemma 4.4, this last term equals

$$
\sum_{\Pi \in \mathcal{P P}_{p}} \prod_{\{i, j\} \in \Pi} m_{2}\left(\nu_{R}\right)\left(\phi_{i}, \phi_{j}\right)=\sum_{\left\{\left\{a_{i}, b_{i}\right\} \mid 1 \leqslant i \leqslant \frac{p}{2}\right\} \in \mathcal{P P}_{p}} \prod_{i=1}^{\frac{p}{2}} m_{2}\left(\nu_{R}\right)\left(\phi_{a_{i}}, \phi_{b_{i}}\right),
$$

which proves the first part of Theorem 1.6.

Let $\phi: \mathbb{R} \rightarrow \mathbb{R}$ be a test-function in the sense of Definition 1.2. Using what we just proved with $\phi_{i}=\phi$ for all $i \in\{1, \ldots, p\}$, we have:

$$
m_{p}\left(\left\langle\nu_{R}, \phi\right\rangle\right)=m_{p}\left(\nu_{R}\right)(\phi, \ldots, \phi)=\operatorname{Card}\left(\mathcal{P P}_{p}\right) \operatorname{Var}\left(\left\langle\nu_{R}, \phi\right\rangle\right)^{\frac{p}{2}}+o\left(R^{\frac{p}{2}}\right) .
$$

Since $\operatorname{Card}\left(\mathcal{P P}_{p}\right)=\mu_{p}$ (see Notation 1.5), this proves the expression of $m_{p}\left(\left\langle\nu_{R}, \phi\right\rangle\right)$ stated in Theorem 1.6. 


\section{Limit theorems}

The purpose of this section is to deduce the functional limit Theorems 1.16 and 1.21 from Theorem 1.6. We prove the Law of Large Numbers (Theorem 1.16) in Section 8.1 and the Central Limit Theorem 1.21 in Section 8.2.

\subsection{Proof of Theorem 1.16: law of large numbers}

This section is concerned with the proof of Theorem 1.16. In the first part of the proof, we derive a Law of Large Numbers for the linear statistics $\left\langle\nu_{R}, \phi\right\rangle$, for a fixed test-function $\phi$. We use the moments estimates of Theorem 1.6 and Markov's Inequality to obtain a quantitative convergence in probability. Then, we deduce the almost sure convergence from the Borel-Cantelli Lemma. In the second part of the proof, we prove a functional Law of Large Number for the random measures $\nu_{R}$. This uses the first part of Theorem 1.16, together with the separability of the space $\mathcal{C}_{c}^{0}(\mathbb{R})$ of continuous functions with compact support equipped with the sup-norm $\|\cdot\|_{\infty}$.

Proof of Theorem 1.16. Let $p \in \mathbb{N}^{*}$ and let $f$ be a normalized stationary centered Gaussian process of class $\mathcal{C}^{2 p}$. Let $\kappa$ denote the correlation function of $f$. We assumed that $\|\kappa\|_{2 p, \eta}=o\left(\eta^{-8 p}\right)$ as $\eta \rightarrow+\infty$, where $\|\cdot\|_{2 p, \eta}$ is defined as in Notation 1.3. Let $\left(R_{n}\right)_{n \in \mathbb{N}}$ be a sequence of positive numbers such that $\sum_{n \in \mathbb{N}} R_{n}^{-p}<+\infty$.

Let $\phi: \mathbb{R} \rightarrow \mathbb{R}$ be a test-function as in Definition 1.2 , we have:

$$
\mathbb{E}\left[\sum_{n \in \mathbb{N}}\left(\frac{1}{R_{n}}\left\langle\nu_{R_{n}}, \phi\right\rangle-\frac{1}{\pi} \int_{\mathbb{R}} \phi(x) \mathrm{d} x\right)^{2 p}\right]=\sum_{n \in \mathbb{N}} \frac{1}{R_{n}^{2 p}} m_{2 p}\left(\left\langle\nu_{R_{n}}, \phi\right\rangle\right) .
$$

We obtain $R_{n}^{-2 p} m_{2 p}\left(\left\langle\nu_{R_{n}}, \phi\right\rangle\right)=O\left(R_{n}^{-p}\right)$ using Proposition 1.11 if $p=1$ and Theorem 1.6 otherwise. Thus, the sum on the right-hand side of the previous equation is finite. This proves that, almost surely, we have:

$$
\sum_{n \in \mathbb{N}}\left(\frac{1}{R_{n}}\left\langle\nu_{R_{n}}, \phi\right\rangle-\frac{1}{\pi} \int_{\mathbb{R}} \phi(x) \mathrm{d} x\right)^{2 p}<+\infty,
$$

hence $\frac{1}{R_{n}}\left\langle\nu_{R_{n}}, \phi\right\rangle \underset{n \rightarrow+\infty}{\longrightarrow} \frac{1}{\pi} \int_{\mathbb{R}} \phi(x) \mathrm{d} x$. This proves our first claim. The almost sure convergence of $\frac{1}{R_{n}} \operatorname{Card}\left(Z \cap\left[0, R_{n}\right]\right)$ is obtained by applying this result with $\phi=\mathbb{1}_{[0,1]}$.

Recall that the space $\mathcal{C}_{c}^{0}(\mathbb{R})$ of continuous functions with compact support is separable for the topology induced by $\|\cdot\|_{\infty}$. Let $\left(\phi_{k}\right)_{k \in \mathbb{N}^{*}}$ denote a dense sequence in $\left(\mathcal{C}_{c}^{0}(\mathbb{R}),\|\cdot\|_{\infty}\right)$. We also denote by $\phi_{0}=\mathbb{1}_{\mathbb{R}}$. For any $N \in \mathbb{N}$, let $\chi_{N}: \mathbb{R} \rightarrow \mathbb{R}$ denote the even continuous function defined by:

$$
\chi_{N}: x \longmapsto \begin{cases}1 & \text { if }|x| \leqslant N, \\ 1-(|x|-N) & \text { if } N \leqslant|x| \leqslant N+1, \\ 0 & \text { if }|x| \geqslant N+1 .\end{cases}
$$

Note that $\phi_{0} \notin \mathcal{C}_{c}^{0}(\mathbb{R})$ but that $\chi_{N} \phi_{0}=\chi_{N} \in \mathcal{C}_{c}^{0}(\mathbb{R})$ for all $N \in \mathbb{N}$.

Using the first part of Theorem 1.16 proved above and the countability of $\mathbb{N}^{2}$, the following happens almost surely:

$$
\forall(k, N) \in \mathbb{N}^{2}, \quad \frac{1}{R_{n}}\left\langle\nu_{R_{n}}, \chi_{N} \phi_{k}\right\rangle \underset{n \rightarrow+\infty}{\longrightarrow} \frac{1}{\pi} \int_{\mathbb{R}} \chi_{N}(x) \phi_{k}(x) \mathrm{d} x .
$$

In the following, we consider a realization of the random process $f$ such that (8.1) holds. For this realization, we will prove by an approximation argument that:

$$
\forall \phi \in \mathcal{C}_{c}^{0}(\mathbb{R}), \quad \frac{1}{R_{n}}\left\langle\nu_{R_{n}}, \phi\right\rangle \underset{n \rightarrow+\infty}{\longrightarrow} \frac{1}{\pi} \int_{\mathbb{R}} \phi(x) \mathrm{d} x,
$$


i.e. that $\frac{1}{R_{n}} \nu_{R_{n}} \underset{n \rightarrow+\infty}{\longrightarrow} \frac{1}{\pi} \mathrm{d} x$ in the weak-* sense in the topological dual of $\left(\mathcal{C}_{c}^{0}(\mathbb{R}),\|\cdot\|_{\infty}\right)$. This yields the result.

Let us consider a realization of $f$ such that (8.1) holds. Let $\phi \in \mathcal{C}_{c}^{0}(\mathbb{R})$ and let $N \in \mathbb{N}$ be large enough that the support of $\phi$ is included in $[-N, N]$. By Equation (8.1) with $k=0$, the non-negative sequence $\left(R_{n}^{-1}\left\langle\nu_{R_{n}}, \chi_{N}\right\rangle\right)_{n \in \mathbb{N}}$ converges towards $\frac{2 N+1}{\pi}$. In particular, this sequence is bounded by some constant $C_{N}>0$.

Let $\varepsilon>0$ and let $k \in \mathbb{N}^{*}$ be such that $\left\|\phi-\phi_{k}\right\|_{\infty} \leqslant \varepsilon$. For all $x \in \mathbb{R}$, we have:

$$
\left|\phi(x)-\chi_{N}(x) \phi_{k}(x)\right|=\left|\chi_{N}(x)\left(\phi(x)-\phi_{k}(x)\right)\right|=\chi_{N}(x)\left|\phi(x)-\phi_{k}(x)\right| \leqslant \varepsilon \chi_{N}(x) .
$$

Then, for all $n \in \mathbb{N}$, we have:

$$
\begin{aligned}
&\left|\frac{1}{R_{n}}\left\langle\nu_{R_{n}}, \phi\right\rangle-\frac{1}{\pi} \int_{\mathbb{R}} \phi(x) \mathrm{d} x\right| \leqslant \frac{1}{R_{n}}\left|\left\langle\nu_{R_{n}}, \phi-\chi_{N} \phi_{k}\right\rangle\right| \\
&+\left|\frac{1}{R_{n}}\left\langle\nu_{R_{n}}, \chi_{N} \phi_{k}\right\rangle-\frac{1}{\pi} \int_{\mathbb{R}} \chi_{N} \phi_{k}(x) \mathrm{d} x\right|+\frac{1}{\pi}\left|\int_{\mathbb{R}} \phi(x)-\chi_{N}(x) \phi_{k}(x) \mathrm{d} x\right| .
\end{aligned}
$$

Using Equation (8.3), the first term on the right-hand side of Equation (8.4) satisfies:

$$
\frac{1}{R_{n}}\left|\left\langle\nu_{R_{n}}, \phi-\chi_{N} \phi_{k}\right\rangle\right| \leqslant \frac{1}{R_{n}}\left\langle\nu_{R_{n}},\left|\phi-\chi_{N} \phi_{k}\right|\right\rangle \leqslant \varepsilon \frac{1}{R_{n}}\left\langle\nu_{R_{n}}, \chi_{N}\right\rangle \leqslant \varepsilon C_{N} .
$$

Similarly, the third term on the right-hand side of Equation (8.4) satisfies:

$$
\begin{aligned}
\frac{1}{\pi}\left|\int_{\mathbb{R}} \phi(x)-\chi_{N}(x) \phi_{k}(x) \mathrm{d} x\right| & \leqslant \frac{1}{\pi} \int_{\mathbb{R}}\left|\phi(x)-\chi_{N}(x) \phi_{k}(x)\right| \mathrm{d} x \leqslant \frac{\varepsilon}{\pi} \int_{\mathbb{R}} \chi_{N}(x) \mathrm{d} x \\
& \leqslant \varepsilon \frac{2 N+1}{\pi} .
\end{aligned}
$$

Using our hypothesis that (8.1) holds for $(k, N)$ the middle term on the right-hand side of Equation (8.4) goes to 0 as $n \rightarrow+\infty$. Finally, for all $n$ large enough we have:

$$
\left|\frac{1}{R_{n}}\left\langle\nu_{R_{n}}, \phi\right\rangle-\frac{1}{\pi} \int_{\mathbb{R}} \phi(x) \mathrm{d} x\right| \leqslant \varepsilon\left(C_{N}+1+\frac{2 N+1}{\pi}\right) .
$$

This proves that Equation (8.2) holds for $\phi$, hence for all $\phi \in \mathcal{C}_{c}^{0}(\mathbb{R})$, as claimed. Thus (8.2) holds almost surely, which concludes the proof.

\subsection{Proof of Theorem 1.21: central limit theorem}

In this section we deduce Theorem 1.21 from the moments estimates of Theorem 1.6. The Central Limit Theorem for a fixed test-function follows from Theorem 1.6 by the method of moments, see [9, Chapter 30]. Then, we obtain the functional Central Limit Theorem by the Lévy-Fernique Theorem, which is a generalization of Lévy's Continuity Theorem for random generalized functions. The result of Fernique [20, Theorem III.6.5] is not exactly what we need, and we refer to [8] instead, for a version of this result that suits us better.

Proof of Theorem 1.21. Let $f$ be a $\mathcal{C}^{\infty}$ centered Gaussian process which is stationary and normalized. Let $\kappa$ denote its correlation function, we assume that $\kappa \in \mathcal{S}(\mathbb{R})$, see Definition 1.18. Let $\sigma$ be the positive constant appearing in the variance asymptotics of Proposition 1.11, which is defined by Equation (1.2).

Let $R>0$, we denote by $\nu_{R}$ the counting measure of $\{x \in \mathbb{R} \mid f(R x)=0\}$. Let $T_{R}$ be the random measure on $\mathbb{R}$ defined by:

$$
T_{R}=\frac{1}{R^{\frac{1}{2}} \sigma}\left(\nu_{R}-\frac{R}{\pi} \mathrm{d} x\right)
$$


where $\mathrm{d} x$ stands for the Lebesgue measure of $\mathbb{R}$. Let $\phi: \mathbb{R} \rightarrow \mathbb{R}$ be a test-function in the sense of Definition 1.2. By Proposition 1.8 and Remark 1.9,

$$
\left\langle T_{R}, \phi\right\rangle=\frac{1}{R^{\frac{1}{2}} \sigma}\left(\left\langle\nu_{R}, \phi\right\rangle-\frac{R}{\pi} \int_{\mathbb{R}} \phi(x) \mathrm{d} x\right)
$$

is an almost-surely well-defined centered random variable. Then, by Theorem 1.6 and Proposition 1.11, for any integer $p \geqslant 2$, we have:

$$
\mathbb{E}\left[\left\langle T_{R}, \phi\right\rangle^{p}\right] \underset{R \rightarrow+\infty}{\longrightarrow} \mu_{p}\|\phi\|_{L^{2}}^{p},
$$

where $\mu_{p}$ is the $p$-th moment of an $\mathcal{N}(0,1)$ random variable, as in Notation 1.5. Hence, we have:

$$
\left\langle T_{R}, \phi\right\rangle \underset{R \rightarrow+\infty}{\longrightarrow} \mathcal{N}\left(0,\|\phi\|_{L^{2}}^{2}\right)
$$

in distribution, by [9, Theorem 30.2]. Note that if $\|\phi\|_{L^{2}}=0$ then $\phi$ vanishes almost everywhere. In this case $\left\langle T_{R}, \phi\right\rangle=0$ almost surely, by Proposition 1.8 and Remark 1.9. This proves the first claim in Theorem 1.21. The Central Limit Theorem follows since $\operatorname{Card}(Z \cap[0, R])=\left\langle\nu_{R}, \mathbb{1}_{[0,1]}\right\rangle$.

Let $R>0$, Lemma 3.12 shows that $T_{R}$ is an almost surely well-defined random element of $\mathcal{S}^{\prime}(\mathbb{R})$. For all $\phi \in \mathcal{S}(\mathbb{R})$, the convergence in distribution of Equation (8.5) and the definition of the standard Gaussian White Noise (cf. Definition 1.20) show that:

$$
\left\langle T_{R}, \phi\right\rangle \underset{R \rightarrow+\infty}{\longrightarrow}\langle W, \phi\rangle .
$$

By [8, Corollary 2.4], we have $T_{R} \underset{R \rightarrow+\infty}{\longrightarrow} W$ in distribution in $\mathcal{S}^{\prime}(\mathbb{R})$. This concludes the proof.

\section{A Examples of smooth non-degenerate processes}

In this section, we build examples of Gaussian processes satisfying the hypotheses of Theorems 1.6, 1.13 and 1.14. First, we need to recall the definition of the spectral measure of a stationary Gaussian process and some of its properties. This is done in Section A.1. In Section A.2, we give a non-degeneracy criterion on the spectral measure for a $\mathcal{C}^{p}$ Gaussian process. Finally, in Section A.3, we give examples of Gaussian processes whose correlation functions lie in the Schwartz space $\mathcal{S}(\mathbb{R})$ of smooth fast-decreasing functions.

\section{A.1 Spectral measure}

This section is concerned with the definition and the properties of the spectral measure of a stationary Gaussian process. Let $f: \mathbb{R} \rightarrow \mathbb{R}$ be a non-zero stationary centered Gaussian process of class $\mathcal{C}^{0}$ and let $\kappa$ denote its correlation function. We assume that $f$ is normalized so that $\operatorname{Var}(f(0))=1$. Then, $\kappa: \mathbb{R} \rightarrow \mathbb{R}$ is a continuous function such that $\kappa(0)=1$. Moreover, $\kappa$ is positive semi-definite, in the sense that, for all $m \in \mathbb{N}^{*}$, for all $x_{1}, \ldots, x_{m} \in \mathbb{R}$, for all $a_{1}, \ldots, a_{m} \in \mathbb{C}$, we have:

$$
\sum_{1 \leqslant j, k \leqslant m} a_{j} \overline{a_{k}} \kappa\left(x_{k}-x_{j}\right)=\mathbb{E}\left[\left|\sum_{j=1}^{m} a_{j} f\left(x_{j}\right)\right|^{2}\right] \geqslant 0 .
$$

By Bochner's Theorem, there exists a unique Borel probability measure $\lambda$ on $\mathbb{R}$ such that $\kappa$ is the characteristic function of $\lambda$, i.e.:

$$
\forall x \in \mathbb{R}, \quad \kappa(x)=\int_{-\infty}^{+\infty} e^{i x \xi} \mathrm{d} \lambda(\xi)
$$


Since $\kappa$ is real-valued, $\lambda$ is symmetric. That is $(-\mathrm{Id})_{*} \lambda=\lambda$, where Id is the identity of $\mathbb{R}$.

Definition A.1 (Spectral measure). Let $f$ be a stationary centered Gaussian process of class $\mathcal{C}^{0}$, normalized so that its correlation function $\kappa$ satisfies $\kappa(0)=1$. The unique symmetric Borel probability measure $\lambda$ such that (A.2) holds is called the spectral measure of $f$.

Conversely, let $\lambda$ be a symmetric Borel probability measure on $\mathbb{R}$ and let $\kappa$ denote its characteristic function. Then, $\kappa$ is a continuous real-valued function such that $\kappa(0)=1$, and $\kappa$ is positive semi-definite (cf. Equation (A.1)). By a theorem of Kolmogorov (see [6, p. 19], for example), there exists a stationary centered Gaussian process $f$ whose correlation function equals $\kappa$.

Let us now relate the properties of the process $f$, its correlation function $\kappa$ and its spectral measure $\lambda$. As explained in Section 2.2, if $f$ is of class $\mathcal{C}^{p}$ for some $p \in \mathbb{N}^{*}$, then $\kappa$ is of class $\mathcal{C}^{2 p}$. In this case, $\lambda$ admits a finite moment of order $2 p$, that is $\int_{\mathbb{R}} \xi^{2 p} \mathrm{~d} \lambda(\xi)<+\infty$. Conversely, if $\lambda$ admits a finite moment of order $2 p$ then $\kappa$ is $\mathcal{C}^{2 p}$. If these conditions are satisfied then, for all $j \in\{0, \ldots, 2 p\}$, for all $x \in \mathbb{R}$, we have:

$$
\kappa^{(j)}(x)=\int_{-\infty}^{+\infty}(i \xi)^{j} e^{i x \xi} \mathrm{d} \lambda(\xi)
$$

The fact that $\kappa$ is of class $\mathcal{C}^{2 p}$ is not enough to ensure that $f$ is a $\mathcal{C}^{p}$-process. However, by Kolmogorov's Theorem [31, Appendix A.9], the process $f$ is of class $\mathcal{C}^{p-1}$, in the sense that there exists a version of $f$ which is of class $\mathcal{C}^{p-1}$. We can say a bit more about the regularity of $f$ :

- for all $\alpha \in(0,1)$, the process $f^{(p-1)}$ is almost surely $\alpha$-Hölder (see [31, Appendix A.11.2]);

- for all $x \in \mathbb{R}$, the variable $f^{(2 p)}(x)$ is well-defined and Gaussian (cf. [6, Proposition 1.13]);

- if there exists $\alpha>0$ such that $\kappa^{(2 p)}(0)-\kappa^{(2 p)}(x)=O\left(|x|^{\alpha}\right)$ as $x \rightarrow 0$, then there exists a version of $f$ of class $\mathcal{C}^{p}$ (cf. [6, Corollary 1.7.b]).

\section{A.2 Non-degeneracy, spectral measure and ergodicity}

In this section, we give a condition on the spectral measure of a stationary Gaussian process implying that the finite-dimensional marginal distributions of this process are non-degenerate. A similar criterion already appeared in [6, p. 64]. Then, we use this result to prove Lemma 2.10 .

Lemma A.2 (Non-degeneracy). Let $f: \mathbb{R} \rightarrow \mathbb{R}$ be a stationary Gaussian process of class $\mathcal{C}^{p}$. Let $\lambda$ denote its spectral measure. If the support of $\lambda$ has an accumulation point in $\mathbb{R}$ then: for all $m \in \mathbb{N}^{*}$, for any $k_{1}, \ldots, k_{m} \in\{0, \ldots, p\}$ and any $x_{1}, \ldots, x_{m} \in \mathbb{R}$ such that the couples $\left(\left(k_{j}, x_{j}\right)\right)_{1 \leqslant j \leqslant m}$ are pairwise distinct, the centered Gaussian vector $\left(f^{\left(k_{j}\right)}\left(x_{j}\right)\right)_{1 \leqslant j \leqslant m}$ is non-degenerate.

Proof. Let us assume that the support of $\lambda$ has an accumulation point in $\mathbb{R}$. Let $m \in \mathbb{N}^{*}$, let $k_{1}, \ldots, k_{m} \in\{0, \ldots, p\}$ and let $x_{1}, \ldots, x_{m} \in \mathbb{R}$ be such that the couples $\left(\left(k_{j}, x_{j}\right)\right)_{1 \leqslant j \leqslant m}$ are pairwise distinct. The Gaussian vector $\left(f^{\left(k_{j}\right)}\left(x_{j}\right)\right)_{1 \leqslant j \leqslant m}$ is non-degenerate if and only if its variance matrix $\Lambda=\left((-1)^{k_{j}} \kappa^{\left(k_{j}+k_{l}\right)}\left(x_{l}-x_{j}\right)\right)_{1 \leqslant i, j \leqslant m}$ is non-singular, that is, if and only if $\operatorname{ker}(\Lambda)=\{0\}$. 
Let $a={ }^{\mathrm{t}}\left(a_{1}, \ldots, a_{m}\right) \in \operatorname{ker}(\Lambda)$, by Equations (2.1) and (A.3), we have:

$$
\begin{aligned}
0={ }^{\mathrm{t}} a \Lambda a & =\sum_{1 \leqslant j, l \leqslant m} a_{j} a_{l}(-1)^{k_{j}} \kappa^{\left(k_{j}+k_{l}\right)}\left(x_{l}-x_{j}\right) \\
& =\sum_{1 \leqslant j, l \leqslant m} a_{j} a_{l} \int_{-\infty}^{+\infty}(i \xi)^{k_{l}}(-i \xi)^{k_{j}} e^{i\left(x_{l}-x_{j}\right) \xi} \mathrm{d} \lambda(\xi) \\
& =\int_{-\infty}^{+\infty}\left|\sum_{j=1}^{m} a_{j}(-i \xi)^{k_{j}} e^{-i x_{j} \xi}\right|^{2} \mathrm{~d} \lambda(\xi) .
\end{aligned}
$$

Hence $g_{a}: \xi \mapsto \sum_{j=1}^{m} a_{j}(-i \xi)^{k_{j}} e^{-i x_{j} \xi}$ vanishes on the support of $\lambda$. Since $g_{a}$ is analytic and the support of $\lambda$ has an accumulation point, we have $g_{a}=0$. Besides, $g_{a}$ is the Fourier transform, in the sense of tempered generalized functions, of $\sum_{j=1}^{m} a_{j}\left(\delta_{x_{j}}\right)^{\left(k_{j}\right)}$, where $\delta_{x}$ stands for the unit Dirac mass at $x \in \mathbb{R}$. Since the Fourier transform is an isomorphism from $\mathcal{S}^{\prime}(\mathbb{R})$ to itself, we have $\sum_{j=1}^{m} a_{j}\left(\delta_{x_{j}}\right)^{\left(k_{j}\right)}=0$. The couples $\left(\left(k_{j}, x_{j}\right)\right)_{1 \leqslant j \leqslant m}$ being pairwise distinct, this implies that $a=0$. Thus $\operatorname{ker}(\Lambda)=\{0\}$ and $\left(f^{\left(k_{j}\right)}\left(x_{j}\right)\right)_{1 \leqslant j \leqslant m}$ is non-degenerate.

We can now prove Lemma 2.10 as a corollary of Lemma A.2.

Proof of Lemma 2.10. Since $\kappa(x) \underset{x \rightarrow+\infty}{\longrightarrow}$, the random process $f$ is ergodic, cf. [1, Theorem 6.5.4]. By the Fomin-Grenander-Maruyama Theorem (see [31, Section 6]), this is equivalent to the fact that the spectral measure $\lambda$ of $f$ has no atom. Note that, in [31], the authors only state one implication in the body of the text, which is not the one we are interested in. The fact that equivalence holds appears as a footnote. Then, any point in the support of $\lambda$ must be an accumulation point. The conclusion follows by Lemma A.2.

\section{A.3 Smooth non-degenerate processes with fast-decreasing correlations}

In this section, we build examples of normalized stationary centered Gaussian $\mathcal{C}^{p}$ processes whose correlation functions, as well as their derivatives, decay as $O\left(x^{-k}\right)$ at infinity.

Lemma A.3. Let $\lambda$ be a probability measure on $\mathbb{R}$ admitting a density $g$ with respect to the Lebesgue measure. Let $k \in \mathbb{N}$ and $p \in \mathbb{N}^{*}$, we assume that $g$ is even, of class $\mathcal{C}^{k}$, and satisfies the following conditions:

1. $\int_{\mathbb{R}} \xi^{2} g(\xi) \mathrm{d} \xi=1$;

2. $\int_{\mathbb{R}} \xi^{2 p+2} g(\xi) \mathrm{d} \xi<+\infty$;

3. for all $j \in\{0, \ldots, k\}, \int_{\mathbb{R}}|\xi|^{p}\left|g^{(j)}(\xi)\right| \mathrm{d} \xi<+\infty$;

4. for all $j \in\{0, \ldots, k-1\},\left|g^{(j)}(\xi)\right|=o\left(|\xi|^{-p}\right)$ as $|\xi| \rightarrow+\infty$.

Then $\lambda$ is the spectral measure of normalized stationary centered Gaussian process $f$ of class $\mathcal{C}^{p}$. Moreover, denoting by $\kappa$ the correlation function of $f$, we have $\kappa^{(j)}(x)=o\left(|x|^{-k}\right)$ as $|x| \rightarrow+\infty$, for all $j \in\{0, \ldots, p\}$.

Proof. Since $g$ is even and continuous, $\lambda$ is a symmetric Borel probability measure. Let $\kappa$ denote the characteristic function of $\lambda$. By Condition 2, the function $\kappa$ is of class $\mathcal{C}^{2 p+2}$. As discussed in Section A.1, this means that $\kappa$ is the correlation function of a stationary centered Gaussian process $f$, at least of class $\mathcal{C}^{p}$. Since $\lambda$ is a probability measure, we have $\kappa(0)=1$. Moreover, by Condition 1 , we have $\kappa^{\prime \prime}(0)=-1$. Thus $f$ is normalized (see Definition 2.9). 
For any $j \in\{0, \ldots, p\}$, an expression of $\kappa^{(j)}$ is given by Equation (A.3). Using Conditions 3 and 4 , we integrate by parts $k$ times, and obtain for any $x \neq 0$ :

$$
\kappa^{(j)}(x)=\frac{i^{j+k}}{x^{k}} \sum_{m=0}^{\min (j, k)}\left(\begin{array}{c}
k \\
m
\end{array}\right) \frac{j !}{(j-m) !} \int_{-\infty}^{+\infty} e^{i x \xi} \xi^{j-m} g^{(k-m)}(\xi) \mathrm{d} \xi .
$$

By Condition 3, the function $\xi \mapsto \xi^{j-m} g^{(k-m)}(\xi)$ is integrable for all $m \leqslant \min (j, k)$. Hence, by the Riemann-Lebesgue Lemma, the integrals in the previous expression tend to 0 as $|x| \rightarrow+\infty$, which shows that $\kappa^{(j)}(x)=o\left(|x|^{-k}\right)$.

Lemmas A.2 and A.3 allow to build examples of Gaussian processes satisfying the hypotheses of our main results, see Theorems 1.6, 1.13 and 1.14. Let us conclude this section by giving a few of them. Recall that the Schwartz space $\mathcal{S}(\mathbb{R})$ is defined by Definition 1.18.

Example A.4. Let $g: \mathbb{R} \rightarrow \mathbb{R}$ be a non-negative continuous even function such that $\int_{\mathbb{R}} \xi^{4} g(\xi) \mathrm{d} \xi<+\infty$ and $\int_{\mathbb{R}} g(\xi) \mathrm{d} \xi=1=\int_{\mathbb{R}} \xi^{2} g(\xi) \mathrm{d} \xi$. Let $\lambda$ denote the measure having the density $g$ with respect to the Lebesgue measure. As explained above, $\lambda$ is the spectral measure of a normalized stationary centered Gaussian $\mathcal{C}^{1}$-process $f$, whose correlation function is denoted by $\kappa$.

- If $g$ is $\mathcal{C}^{1}$ and we have $g(\xi)=O\left(\xi^{-8}\right)$ and $g^{\prime}(\xi)=O\left(\xi^{-4}\right)$ as $\xi \rightarrow+\infty$, then $f$ is $\mathcal{C}^{2}$ and $\kappa(x), \kappa^{\prime}(x)$ and $\kappa^{\prime \prime}(x)$ are $o\left(|x|^{-1}\right)$ as $x \rightarrow+\infty$. In particular, $f$ satisfies the hypotheses of Proposition 1.11.

- Let $p \in \mathbb{N}^{*}$, if $g(\xi)=O\left(\xi^{-2 p-4}\right)$ as $\xi \rightarrow+\infty$ then $f$ is of class $\mathcal{C}^{p}$ and $\kappa^{(j)}(x) \underset{x \rightarrow+\infty}{\longrightarrow} 0$ for all $j \in\{0, \ldots, p\}$. In particular, $f$ satisfies the hypotheses of Theorems 1.13 and 1.14 .

- If $g \in \mathcal{S}(\mathbb{R})$, then $f$ is $\mathcal{C}^{\infty}$ and $\kappa \in \mathcal{S}(\mathbb{R})$. Hence $f$ satisfies the hypotheses of Proposition 1.11 and Theorems 1.6, 1.13 and 1.14.

- If $g: \xi \mapsto \frac{1}{\sqrt{2 \pi}} \exp \left(-\frac{1}{2} \xi^{2}\right)$ is the standard Gaussian density, then $\kappa: x \mapsto \exp \left(-\frac{1}{2} x^{2}\right)$ and $f$ is the Bargmann-Fock process discussed in Section 1.2.

- If $g=\frac{1}{2 \sqrt{3}} \mathbb{1}_{[-\sqrt{3}, \sqrt{3}]}$ then $\kappa: x \mapsto \operatorname{sinc}(\sqrt{3} x)$, where sinc : $x \mapsto \sum_{k \geqslant 0} \frac{(-1)^{k} x^{2 k}}{(2 k+1) !}$ is the smooth extension of $x \mapsto \frac{1}{x} \sin (x)$ to $\mathbb{R}$. The density $g$ is not regular enough to apply Lemma A.3, but $\kappa$ is still the correlation function of a normalized stationary centered Gaussian process $f$ of class $\mathcal{C}^{\infty}$ (cf. Section A.1). By Lemma A.2, $f$ satisfies the hypotheses of Theorem 1.13. Moreover, one can check that $\kappa$ and all its derivatives are square-integrable on $\mathbb{R}$ and tend to 0 at infinity, hence $f$ satisfies the hypotheses of Proposition 1.11 and Theorem 1.14 for any $p \in \mathbb{N}^{*}$.

- If $g: \xi \mapsto \frac{1}{\sqrt{2}} \exp (-\sqrt{2}|\xi|)$, then $\kappa: x \mapsto\left(1+\frac{x^{2}}{2}\right)^{-1}$ (one can check this using his favorite computational software). As above $g$ is not regular enough to apply Lemma A. 3 directly. Yet, $\kappa$ is the correlation function of a normalized stationary centered Gaussian $\mathcal{C}^{\infty}$-process satisfying the hypotheses of Proposition 1.11 and Theorems 1.13 and 1.14 for any $p \in \mathbb{N}^{*}$.

Remark A.5. Let $h$ be a non-negative even function. If we have $\int_{\mathbb{R}} h(\xi) \mathrm{d} \xi=A>0$ and $\int_{\mathbb{R}} \xi^{2} h(\xi) \mathrm{d} \xi=B$, then $g: \xi \mapsto \sqrt{\frac{B}{A^{3}}} h\left(\sqrt{\frac{B}{A}} \xi\right)$ is such that $\int_{R} g(\xi) \mathrm{d} \xi=1=\int_{\mathbb{R}} \xi^{2} g(\xi) \mathrm{d} \xi$. Moreover, $g$ is non-negative, even and it has the same regularity and asymptotic behavior as $h$. 


\section{B Properties of the density function $F$}

\section{B.1 Proof of Lemma 4.2}

The goal of this section is to prove Lemma 4.2, which gives an expression of the function $F$ defined by Definition 4.1. Recall that in Lemma 4.2 we work under the hypotheses of Proposition 1.11. In particular, $f$ is a $\mathcal{C}^{2}$ Gaussian process whose correlation function $\kappa$ tends to 0 at infinity.

Lemma B.1. For all $z>0$ we have $N_{2}(0, z)=\frac{2}{\pi} b(z)\left(\sqrt{1-a(z)^{2}}+a(z) \arcsin (a(z))\right)$, where

$$
a(z)=\frac{\kappa(z) \kappa^{\prime}(z)^{2}-\kappa(z)^{2} \kappa^{\prime \prime}(z)+\kappa^{\prime \prime}(z)}{1-\kappa(z)^{2}-\kappa^{\prime}(z)^{2}} \quad \text { and } \quad b(z)=1-\frac{\kappa^{\prime}(z)^{2}}{1-\kappa(z)^{2}} .
$$

Moreover, $|a(z)| \leqslant 1$.

Proof. Let $z>0$. The random vector $\left(f(0), f(z), f^{\prime}(0), f^{\prime}(z)\right)$ is a centered Gaussian in $\mathbb{R}^{4}$ whose variance matrix equals:

$$
\left(\begin{array}{cccc}
1 & \kappa(z) & 0 & \kappa^{\prime}(z) \\
\kappa(z) & 1 & -\kappa^{\prime}(z) & 0 \\
0 & -\kappa^{\prime}(z) & 1 & -\kappa^{\prime \prime}(z) \\
\kappa^{\prime}(z) & 0 & -\kappa^{\prime \prime}(z) & 1
\end{array}\right)
$$

Since $\kappa$ tends to 0 at infinity, by Lemma 2.10 the Gaussian vector $(f(0), f(z))$ is nondegenerate, that is $\kappa(z)^{2}<1$. Then, by [6, Proposition 1.2], $\left(f^{\prime}(0), f^{\prime}(z)\right)$ given that $f(0)=0=f(z)$ is a centered Gaussian vector in $\mathbb{R}^{2}$. Moreover, its variance matrix is:

$$
\begin{aligned}
\Lambda(z) & =\left(\begin{array}{cc}
1 & -\kappa^{\prime \prime}(z) \\
-\kappa^{\prime \prime}(z) & 1
\end{array}\right)-\left(\begin{array}{cc}
0 & -\kappa^{\prime}(z) \\
\kappa(z) & 0
\end{array}\right)\left(\begin{array}{cc}
1 & \kappa(z) \\
\kappa(z) & 1
\end{array}\right)^{-1}\left(\begin{array}{cc}
0 & \kappa^{\prime}(z) \\
-\kappa^{\prime}(z) & 0
\end{array}\right) \\
& =\left(\begin{array}{ll}
b(z) & c(z) \\
c(z) & b(z)
\end{array}\right),
\end{aligned}
$$

where

$$
b(z)=1-\frac{\kappa^{\prime}(z)^{2}}{1-\kappa(z)^{2}} \quad \text { and } \quad c(z)=-\kappa^{\prime \prime}(z)-\frac{\kappa(z) \kappa^{\prime}(z)^{2}}{1-\kappa(z)^{2}} .
$$

Note that, since $\Lambda(z)$ is a variance matrix, we have $b(z) \geqslant|c(z)| \geqslant 0$.

Let $(X, Y) \sim \mathcal{N}(0, \Lambda(z))$ in $\mathbb{R}^{2}$, we have $N_{2}(0, z)=\mathbb{E}[|X||Y|]$. If $b(z)>|c(z)|$, then $\Lambda(z)$ is invertible and $\Lambda^{-1}(z)=\left(\begin{array}{cc}A(z) & B(z) \\ B(z) & A(z)\end{array}\right)$, where

$$
A(z)=\frac{b(z)}{b(z)^{2}-c(z)^{2}} \quad \text { and } \quad B(z)=-\frac{c(z)}{b(z)^{2}-c(z)^{2}} .
$$

Then, using [11, Equation (A.1)], we have:

$$
\begin{aligned}
\mathbb{E}[|X||Y|] & =\frac{1}{2 \pi \sqrt{b(z)^{2}-c(z)^{2}}} \int_{\mathbb{R}^{2}}|x||y| \exp \left(-A(z) \frac{x^{2}+y^{2}}{2}-B(z) x y\right) \mathrm{d} x \mathrm{~d} y \\
& =\frac{2}{\pi} b(z)\left(\sqrt{1-\frac{c(z)^{2}}{b(z)^{2}}}+\frac{c(z)}{b(z)} \arcsin \left(\frac{c(z)}{b(z)}\right)\right)
\end{aligned}
$$

On the other hand, if $b(z)=|c(z)|$ then $|X|=|Y|$ almost surely. Hence,

$$
\mathbb{E}[|X||Y|]=\mathbb{E}\left[X^{2}\right]=b(z)=\frac{2}{\pi} b(z)\left(\sqrt{1-\frac{c(z)^{2}}{b(z)^{2}}}+\frac{c(z)}{b(z)} \arcsin \left(\frac{c(z)}{b(z)}\right)\right) .
$$


To conclude, note that $a(z)=-\frac{c(z)}{b(z)}$, so that $|a(z)| \leqslant 1$ and:

$$
N_{2}(0, z)=\mathbb{E}[|X||Y|]=\frac{2}{\pi} b(z)\left(\sqrt{1-a(z)^{2}}+a(z) \arcsin (a(z))\right) .
$$

Proof of Lemma 4.2. By definition of $F$ and $\rho_{2}$ (see Definitions 4.1 and 3.1), for all $z \neq 0$, we have:

$$
F(z)=\frac{1}{2 \pi} \frac{N_{2}(0, z)}{D_{2}(0, z)^{\frac{1}{2}}}-\frac{1}{\pi^{2}} .
$$

Note that, $N_{2}$ and $D_{2}$ are symmetric functions on $\mathbb{R}^{2} \backslash \Delta_{2}$. Then, using the stationarity of $f$, we have $N_{2}(0, z)=N_{2}(z, 0)=N_{2}(0,-z)$, and similarly $D_{2}(0, z)=D_{2}(0,-z)$. Thus $F(z)=F(-z)$ for all $z \neq 0$.

Let $z>0$, we have $D_{2}(0, z)=1-\kappa(z)^{2}$ (see Example 3.3) and the expression of $N_{2}(0, z)$ is given by Lemma B.1. Then, a direct computation yields:

$$
F(z)=\frac{1}{\pi^{2}}\left(\frac{1-\kappa(z)^{2}-\kappa^{\prime}(z)^{2}}{\left(1-\kappa(z)^{2}\right)^{\frac{3}{2}}}\left(\sqrt{1-a(z)^{2}}+a(z) \arcsin (a(z))\right)-1\right),
$$

where $a(z)$ is defined as in Lemmas 4.2 and B.1. In particular, $|a(z)| \leqslant 1$.

\section{B.2 Proof of Lemma 4.3}

In this section, we prove the integrability of the function $F$ defined by Definition 4.1 , under the hypotheses of Proposition 1.11.

Lemma B.2. Under the hypotheses of Proposition 1.11, we have $F(z) \underset{z \rightarrow 0}{\longrightarrow}-\frac{1}{\pi^{2}}$.

Proof. Let us consider the expression of $F$ derived in Lemma 4.2. Note that, for all $z>0$, we have:

$$
0 \leqslant \sqrt{1-a(z)^{2}}+a(z) \arcsin (a(z)) \leqslant 1+\frac{\pi}{2} .
$$

Hence, it is enough to prove that:

$$
\frac{1-\kappa(z)^{2}-\kappa^{\prime}(z)^{2}}{\left(1-\kappa(z)^{2}\right)^{\frac{3}{2}}} \underset{z \rightarrow 0}{\longrightarrow} 0
$$

We know that $\kappa$ is $\mathcal{C}^{4}$. Moreover, $\kappa(0)=1=-\kappa^{\prime \prime}(0)$ and $\kappa^{\prime}(0)=0=\kappa^{(3)}(0)$. Thus, as $z \rightarrow 0$, we have:

$$
\kappa(z)=1-\frac{z^{2}}{2}+O\left(z^{4}\right) \quad \text { and } \quad \kappa^{\prime}(z)=-z+O\left(z^{3}\right) .
$$

These estimates yield that $\frac{1-\kappa(z)^{2}-\kappa^{\prime}(z)^{2}}{\left(1-\kappa(z)^{2}\right)^{\frac{3}{2}}}=O(z)$ as $z \rightarrow 0$, which concludes the proof.

Lemma B.3. Under the hypotheses of Proposition 1.11, as $z \rightarrow+\infty$, we have:

$$
F(z)=O\left(\kappa(z)^{2}+\kappa^{\prime}(z)^{2}+\kappa^{\prime \prime}(z)^{2}\right) .
$$

Proof. Once again, we use the expression of $F$ derived in Lemma 4.2. First, note that since $\kappa(z), \kappa^{\prime}(z)$ and $\kappa^{\prime \prime}(z)$ tend to 0 as $z \rightarrow+\infty$, we have: $a(z)=O(\kappa(z))+O\left(\kappa^{\prime \prime}(z)\right)$ as $z \rightarrow+\infty$. Then,

$$
\sqrt{1-a(z)^{2}}+a(z) \arcsin (a(z))=1+O\left(a(z)^{2}\right)=1+O\left(\kappa(z)^{2}\right)+O\left(\kappa^{\prime \prime}(z)^{2}\right),
$$


as $z \rightarrow+\infty$. On the other hand, as $z \rightarrow+\infty$, we have:

$$
\frac{1-\kappa(z)^{2}-\kappa^{\prime}(z)^{2}}{\left(1-\kappa(z)^{2}\right)^{\frac{3}{2}}}=1+O\left(\kappa(z)^{2}\right)+O\left(\kappa^{\prime}(z)^{2}\right) .
$$

These two estimates yield that $F(z)=O\left(\kappa(z)^{2}\right)+O\left(\kappa^{\prime}(z)^{2}\right)+O\left(\kappa^{\prime \prime}(z)^{2}\right)$ as $z \rightarrow+\infty$.

Proof of Lemma 4.3. First, note that $F$ is well-defined and continuous on $\mathbb{R} \backslash\{0\}$. Indeed, $\rho_{2}$ is continuous on $\mathbb{R}^{2} \backslash \Delta_{2}$ by Lemma 3.9. By Lemma B.2, $F(z) \underset{z \rightarrow 0}{\longrightarrow}-\frac{1}{\pi^{2}}$. In particular, $F$ is integrable near 0 . Since $F$ is even and $\kappa(z), \kappa^{\prime}(z)$ and $\kappa^{\prime \prime}(z)$ tend to 0 as $z \rightarrow+\infty$, we have $F(z) \underset{|z| \rightarrow+\infty}{\longrightarrow} 0$, by Lemma B.3.

Under the hypotheses of Proposition 1.11, both $\kappa$ and $\kappa^{\prime \prime}$ are square-integrable. By an integration by parts and the Cauchy-Schwarz Inequality, this implies that $\kappa^{\prime}$ is also square-integrable. Finally, $F$ is integrable at infinity by Lemma B.3.

\section{A Gaussian lemma}

In this appendix, we prove an estimate that we used in our study of the Kac-Rice numerators $N_{\mathcal{I}}$ (see Definition 6.14). More precisely, Corollary C.3 below is used in the proofs of Lemmas 6.16 and 6.36 .

Let $k \in \mathbb{N}^{*}$, we denote by $\operatorname{Sym}_{k}(\mathbb{R})$ the space of symmetric matrices of size $k$ with real coefficients and by $\operatorname{Sym}_{k}^{+}(\mathbb{R}) \subset \operatorname{Sym}_{k}(\mathbb{R})$ the subset of positive semi-definite matrices. We equip $\operatorname{Sym}_{k}(\mathbb{R})$ with the sup-norm $\|\cdot\|_{\infty}$, see Notation 6.25.

Definition C.1. Let $U \in \operatorname{Sym}_{k}^{+}(\mathbb{R})$ and let $\left(X_{1}, \ldots, X_{k}\right) \sim \mathcal{N}(0, U)$, we denote by

$$
\Pi_{k}(U)=\mathbb{E}\left[\prod_{i=1}^{k}\left|X_{i}\right|\right]
$$

Lemma C.2. Let $k \in \mathbb{N}^{*}$, there exists $C_{k}>0$ such that, for all $U$ and $V \in \operatorname{Sym}_{k}^{+}(\mathbb{R})$ :

$$
\left|\Pi_{k}(V)-\Pi_{k}(U)\right| \leqslant C_{k}\|V-U\|_{\infty}^{\frac{1}{2}}\left(\max \left(\|U\|_{\infty},\|V\|_{\infty}\right)\right)^{\frac{k-1}{2}} .
$$

Proof. Let $\left(X_{1}, \ldots, X_{k}\right) \sim \mathcal{N}(0, U)$ and $\left(Y_{1}, \ldots, Y_{k}\right) \sim \mathcal{N}(0, V)$ be centered Gaussian vectors in $\mathbb{R}^{k}$ of variance matrices $U$ and $V$, respectively.

Let us first assume that $V-U \in \operatorname{Sym}_{k}^{+}(\mathbb{R})$ and let $T=\left(T_{1}, \ldots, T_{k}\right) \sim \mathcal{N}(0, V-U)$ be independent of $X$. In this case $X+T \sim \mathcal{N}(0, V)$, and we can assume that $Y=X+T$ without loss of generality. Then,

$$
\begin{aligned}
\left|\Pi_{k}(V)-\Pi_{k}(U)\right| & =\left|\mathbb{E}\left[\prod_{i=1}^{k}\left|Y_{i}\right|\right]-\mathbb{E}\left[\prod_{i=1}^{k}\left|X_{i}\right|\right]\right| \\
& \leqslant \mathbb{E}\left[\left|\prod_{i=1}^{k} Y_{i}-\prod_{i=1}^{k} X_{i}\right|\right] \\
& \leqslant \sum_{j=1}^{k} \mathbb{E}\left[\left(\prod_{i=1}^{j-1}\left|Y_{i}\right|\right)\left|Y_{j}-X_{j}\right|\left(\prod_{i=j+1}^{k}\left|X_{i}\right|\right)\right] \\
& \leqslant \sum_{j=1}^{k} \prod_{i=1}^{j-1}\left(\mathbb{E}\left[\left|Y_{i}\right|^{k}\right]^{\frac{1}{k}}\right) \mathbb{E}\left[\left|T_{j}\right|^{k}\right]^{\frac{1}{k}}\left(\prod_{i=j+1}^{k} \mathbb{E}\left[\left|X_{i}\right|^{k}\right]^{\frac{1}{k}}\right),
\end{aligned}
$$

where we obtained the last line by applying Hölder's Inequality. Now, for all $i \in\{1, \ldots, k\}$, we have:

$$
\mathbb{E}\left[\left|X_{i}\right|^{k}\right] \leqslant \mathbb{E}\left[\left(X_{i}\right)^{2 k}\right]^{\frac{1}{2}}=\left(\mu_{2 k}\right)^{\frac{1}{2}} \operatorname{Var}\left(X_{i}\right)^{\frac{k}{2}} \leqslant\left(\mu_{2 k}\right)^{\frac{1}{2}}\|U\|_{\infty}^{\frac{k}{2}},
$$


where $\mu_{2 k}$ stands for the $2 k$-th moment of an $\mathcal{N}(0,1)$ real variable, as in Notation 1.5. Similarly, we have $\mathbb{E}\left[\left|Y_{i}\right|^{k}\right] \leqslant\left(\mu_{2 k}\right)^{\frac{1}{2}}\|V\|_{\infty}^{\frac{k}{2}}$ and $\mathbb{E}\left[\left|T_{i}\right|^{k}\right] \leqslant\left(\mu_{2 k}\right)^{\frac{1}{2}}\|V-U\|_{\infty}^{\frac{k}{2}}$. Hence,

$$
\left|\Pi_{k}(V)-\Pi_{k}(U)\right| \leqslant k\left(\mu_{2 k}\right)^{\frac{1}{2}}\|V-U\|_{\infty}^{\frac{1}{2}}\left(\max \left(\|U\|_{\infty},\|V\|_{\infty}\right)\right)^{\frac{k-1}{2}} .
$$

This concludes the proof in the special case where $V-U$ is positive semi-definite.

Let us now consider the general case and let us denote by $\varepsilon=\|V-U\|_{\infty}$. Let $\operatorname{Id}_{k}$ denote the identity matrix of size $k$ and $W=U+k \varepsilon \operatorname{Id}_{k}$. Then, $W-U=k \varepsilon \operatorname{Id}_{k} \in \operatorname{Sym}_{k}^{+}(\mathbb{R})$. Moreover, we have $W-V=k \varepsilon \operatorname{Id}_{k}+U-V$. Since for all $x=\left(x_{i}\right)_{1 \leqslant i \leqslant k} \in \mathbb{R}^{k}$, we have

$$
\left|{ }^{\mathrm{t}} x(U-V) x\right| \leqslant \varepsilon \sum_{1 \leqslant i, j \leqslant k}\left|x_{i}\right|\left|x_{j}\right|=\varepsilon\left(\sum_{i=1}^{k}\left|x_{i}\right|\right)^{2} \leqslant k \varepsilon \sum_{i=1}^{k} x_{i}^{2},
$$

the matrix $W-V$ is also positive semi-definite. Let $Z=\left(Z_{1}, \ldots, Z_{k}\right) \sim \mathcal{N}(0, W)$, using Equation (C.1), we obtain:

$$
\begin{aligned}
& \left|\Pi_{k}(V)-\Pi_{k}(U)\right| \leqslant\left|\Pi_{k}(W)-\Pi_{k}(V)\right|+\left|\Pi_{k}(W)-\Pi_{k}(U)\right| \\
& \quad \leqslant k\left(\mu_{2 k}\right)^{\frac{1}{2}}\left(\|W-U\|_{\infty}^{\frac{1}{2}}+\|W-V\|_{\infty}^{\frac{1}{2}}\right)\left(\max \left(\|U\|_{\infty},\|V\|_{\infty},\|W\|_{\infty}\right)\right)^{\frac{k-1}{2}} .
\end{aligned}
$$

We know that $\|W-V\|_{\infty} \leqslant\|W-U\|_{\infty}+\|V-U\|_{\infty}$. Hence, by definition of $W$ and $\varepsilon$, we get $\|W-U\|_{\infty}=k \varepsilon=k\|V-U\|_{\infty}$ and $\|W-V\|_{\infty} \leqslant(k+1)\|V-U\|_{\infty}$. Moreover,

$$
\|W\|_{\infty} \leqslant\|U\|_{\infty}+\|W-U\|_{\infty}=\|U\|_{\infty}+k\|V-U\|_{\infty} \leqslant(2 k+1) \max \left(\|U\|_{\infty},\|V\|_{\infty}\right) .
$$

Finally, setting $C_{k}=k(2 k+1)^{\frac{k+1}{2}}\left(\mu_{2 k}\right)^{\frac{1}{2}}$, we have:

$$
\left|\Pi_{k}(V)-\Pi_{k}(U)\right| \leqslant C_{k}\|V-U\|_{\infty}^{\frac{1}{2}}\left(\max \left(\|U\|_{\infty},\|V\|_{\infty}\right)\right)^{\frac{k-1}{2}} .
$$

Corollary C.3 (Regularity). Let $k \in \mathbb{N}^{*}$, the $\operatorname{map} \Pi_{k}: \operatorname{Sym}_{k}^{+}(\mathbb{R}) \rightarrow \mathbb{R}$ defined by Definition C.1 is $\frac{1}{2}$-Hölder on compact subsets of $\operatorname{Sym}_{k}^{+}(\mathbb{R})$, for the sup-norm $\|\cdot\|_{\infty}$. In particular, the map $\Pi_{k}$ is continuous.

Proof. Let $K \subset \operatorname{Sym}_{k}^{+}(\mathbb{R})$ be a compact subset, we denote by $M=\max _{U \in K}\|U\|_{\infty}$. By Lemma C.2, for all $U, V \in K$, we have $\left|\Pi_{k}(V)-\Pi_{k}(U)\right| \leqslant C_{k} M^{\frac{k-1}{2}}\|V-U\|_{\infty}^{\frac{1}{2}}$. Thus, $\Pi_{k}$ is $\frac{1}{2}$-Hölder on compact subsets of $\operatorname{Sym}_{k}^{+}(\mathbb{R})$, hence continuous.

\section{References}

[1] Robert J. Adler, The geometry of random fields, Classics in Applied Mathematics, vol. 62, Society for Industrial and Applied Mathematics (SIAM), Philadelphia, PA, 2010, Reprint of the 1981 original. MR-3396215.

[2] Robert J. Adler and Jonathan E. Taylor, Random fields and geometry, 1st ed., Monographs in Mathematics, Springer, New York, 2007. MR-2319516.

[3] Michele Ancona, Random sections of line bundles over real Riemann surfaces, Int. Math. Res. Not. IMRN (2021), no. 9, 7004-7059. MR-4251296.

[4] Michele Ancona and Thomas Letendre, Roots of Kostlan polynomials: moments, strong Law of Large Numbers and Central Limit Theorem, Ann. H. Lebesgue (2021), to be published. arXiv:1911.12182.

[5] Diego Armentano, Jean-Marc Azaïs, Federico Dalmao, José R. Leòn, and Ernesto Mordecki, On the finiteness of the moments of the measure of level sets of random fields, (2019). arXiv:1909.10243. 
[6] Jean-Marc Azaïs and Mario Wschebor, Level sets and extrema of random processes and fields, John Wiley \& Sons, Hoboken, NJ, 2009. MR-2478201.

[7] Riddhipratim Basu, Amir Dembo, Naomi Feldheim, and Ofer Zeitouni, Exponential concentration for zeroes of stationary Gaussian processes, Int. Math. Res. Not. IMRN (2020), no. 23, 9769-9796. MR-4182810.

[8] Hermine Biermé, Olivier Durieu, and Yizao Wang, Generalized random fields and Lévy's continuity theorem on the space of tempered distributions, Commun. Stoch. Anal. 12 (2018), no. 4, 427-445. MR-3957708

[9] Patrick Billingsley, Probability and measure, 3rd ed., Wiley Series in Probability and Mathematical Statistics, John Wiley \& Sons, New York, 1995. MR-1324786

[10] Bartłomiej Błaszczyszyn, Dhandapani Yogeshwaran, and Joseph E. Yukich, Limit theory for geometric statistics of point processes having fast decay of correlations, Ann. Probab. 47 (2019), no. 2, 835-895. MR-3916936

[11] Pavel Bleher and Xiaojun Di, Correlations between zeros of a random polynomial, J. Statist. Phys. 88 (1997), no. 1-2, 269-305. MR-1468385

[12] Pavel Bleher, Bernard Shiffman, and Steve Zelditch, Universality and scaling of correlations between zeros on complex manifolds, Invent. Math. 142 (2000), no. 2, 351-395. MR-1794066

[13] Harald Cramér and Malcolm R. Leadbetter, The moments of the number of crossings of a level by a stationary normal process, Ann. Math. Statist. 36 (1965), 1656-1663. MR-185682

[14] Jack Cuzick, Conditions for finite moments of the number of zero crossings for Gaussian processes, Ann. Probability 3 (1975), no. 5, 849-858. MR-388515

[15] Jack Cuzick, A central limit theorem for the number of zeros of a stationary Gaussian process, Ann. Probability 4 (1976), no. 4, 547-556. MR-420809

[16] Jack Cuzick, Local nondeterminism and the zeros of Gaussian processes, Ann. Probability 6 (1978), no. 1, 72-84. MR-488252

[17] Federico Dalmao, Asymptotic variance and CLT for the number of zeros of Kostlan Shub Smale random polynomials, C. R. Math. Acad. Sci. Paris 353 (2015), no. 12, 1141-1145. MR-3427922

[18] Yen Do and Van Vu, Central limit theorems for the real zeros of Weyl polynomials, Amer. J. Math. 142 (2020), no. 5, 1327-1369. MR-4150647

[19] François Dubeau, On Hermite interpolation and divided differences, Surv. Math. Appl. 15 (2020), 257-279. MR-4099738

[20] Xavier Fernique, Processus linéaires, processus généralisés, Ann. Inst. Fourier 17 (1967), no. 1, 1-92. MR-221576

[21] Damien Gayet and Jean-Yves Welschinger, Exponential rarefaction of real curves with many components, Publ. Math. Inst. Hautes Études Sci. (2011), no. 113, 69-96. MR-2805598

[22] Donald Geman, On the variance of the number of zeros of a stationary Gaussian process, Ann. Math. Statist. 43 (1972), 977-982. MR-301791

[23] Mark Kac, On the average number of real roots of a random algebraic equation, Bull. Amer. Math. Soc. 49 (1943), 314-320. MR-7812

[24] Eric Kostlan, On the distribution of roots of random polynomials, From Topology to Computation: Proceedings of the Smalefest (Berkeley, CA, 1990), Springer, New York, 1993, pp. 419-431. MR-1246137

[25] Marie F. Kratz, Level crossings and other level functionals of stationary Gaussian processes, Probab. Surv. 3 (2006), 230-288. MR-2264709

[26] Marie F. Kratz and José R. Leòn, Hermite polynomial expansion for non-smooth functionals of stationary Gaussian processes: crossings and extremes, Stochastic Process. Appl. 66 (1997), no. 2, 237-252. MR-1440400

[27] Marie F. Kratz and José R. Leòn, Central limit theorems for level functionals of stationary Gaussian processes and fields, J. Theoret. Probab. 14 (2001), no. 3, 639-672. MR-1860517

[28] Raphaël Lachièze-Rey, Variance linearity for real Gaussian zeros, (2020). arXiv:2006.10341. 
[29] Thomas Letendre and Martin Puchol, Variance of the volume of random real algebraic submanifolds II, Indiana Univ. Math. J. 68 (2019), no. 6, 1649-1720. MR-4052739

[30] Fedor L. Nazarov and Mikhail L. Sodin, Correlation functions for random complex zeroes: strong clustering and local universality, Comm. Math. Phys. 310 (2012), no. 1, 75-98. MR2885614

[31] Fedor L. Nazarov and Mikhail L. Sodin, Asymptotic laws for the spatial distribution and the number of connected components of zero sets of Gaussian random functions, Zh. Mat. Fiz. Anal. Geom. 12 (2016), no. 3, 205-278. MR-3522141

[32] Vladimir I. Piterbarg, Asymptotic methods in the theory of Gaussian processes and fields, Translations of Mathematical Monographs, vol. 148, American Mathematical Society, Providence, RI, 1996, Translated from the Russian by V. V. Piterbarg. MR-1361884

[33] Stephen O. Rice, Mathematical analysis of random noise, Bell System Tech. J. 23 (1944), 282-332. MR-10932

[34] Bernard Shiffman and Steve Zelditch, Distribution of zeros of random and quantum chaotic sections of positive line bundles, Comm. Math. Phys. 200 (1999), no. 3, 661-683. MR-1675133

[35] Donald N. Ylvisaker, The expected number of zeros of a stationary Gaussian process, Ann. Math. Statist. 36 (1965), 1043-1046. MR-177458

Acknowledgments. Thomas Letendre thanks Julien Fageot for useful discussions about Fernique's Theorem, Benoit Laslier for his help in the proof of Lemma C.2 and Hugo Vanneuville for pointing out the relation between ergodicity and decay of correlations. The authors are grateful to Damien Gayet for suggesting they write this paper in the first place, and to Misha Sodin for bringing to their attention the intrinsic interest of clustering properties for $k$-point functions. They also thank Jean-Yves Welschinger for his support and Louis Gass for spotting an error in an earlier version of Lemma 7.8. Finally, the authors thank the anonymous referee for their careful reading of the paper, and their comments that helped to improve the exposition of the main results. 


\section{Electronic Journal of Probability Electronic Communications in Probability}

\section{Advantages of publishing in EJP-ECP}

- Very high standards

- Free for authors, free for readers

- Quick publication (no backlog)

- Secure publication $\left(\mathrm{LOCKSS}^{1}\right)$

- Easy interface (EJMS²)

\section{Economical model of EJP-ECP}

- Non profit, sponsored by $\mathrm{IMS}^{3}, \mathrm{BS}^{4}$, ProjectEuclid ${ }^{5}$

- Purely electronic

\section{Help keep the journal free and vigorous}

- Donate to the IMS open access fund ${ }^{6}$ (click here to donate!)

- Submit your best articles to EJP-ECP

- Choose EJP-ECP over for-profit journals

\footnotetext{
${ }^{1}$ LOCKSS: Lots of Copies Keep Stuff Safe http://www. lockss.org/

${ }^{2}$ EJMS: Electronic Journal Management System http://www.vtex.lt/en/ejms.html

${ }^{3}$ IMS: Institute of Mathematical Statistics http://www.imstat.org/

${ }^{4}$ BS: Bernoulli Society http://www. bernoulli-society.org/

${ }^{5}$ Project Euclid: https://projecteuclid.org/

${ }^{6}$ IMS Open Access Fund: http://www.imstat.org/publications/open.htm
} 